\title{
Too scared to achieve
}

Citation for published version (APA):

Wehner, C. (2018). Too scared to achieve: the relation between neuroticism, conscientiousness and socioeconomic outcomes. [Doctoral Thesis, Maastricht University]. Boekenplan Maastricht. https://doi.org/10.26481/dis.20180525cw

Document status and date:

Published: 01/01/2018

DOI:

$10.26481 /$ dis.20180525cw

Document Version:

Publisher's PDF, also known as Version of record

\section{Please check the document version of this publication:}

- A submitted manuscript is the version of the article upon submission and before peer-review. There can be important differences between the submitted version and the official published version of record.

People interested in the research are advised to contact the author for the final version of the publication, or visit the DOI to the publisher's website.

- The final author version and the galley proof are versions of the publication after peer review.

- The final published version features the final layout of the paper including the volume, issue and page numbers.

Link to publication

\footnotetext{
General rights rights.

- You may freely distribute the URL identifying the publication in the public portal. please follow below link for the End User Agreement:

www.umlib.nl/taverne-license

Take down policy

If you believe that this document breaches copyright please contact us at:

repository@maastrichtuniversity.nl

providing details and we will investigate your claim.
}

Copyright and moral rights for the publications made accessible in the public portal are retained by the authors and/or other copyright owners and it is a condition of accessing publications that users recognise and abide by the legal requirements associated with these

- Users may download and print one copy of any publication from the public portal for the purpose of private study or research.

- You may not further distribute the material or use it for any profit-making activity or commercial gain

If the publication is distributed under the terms of Article $25 \mathrm{fa}$ of the Dutch Copyright Act, indicated by the "Taverne" license above, 

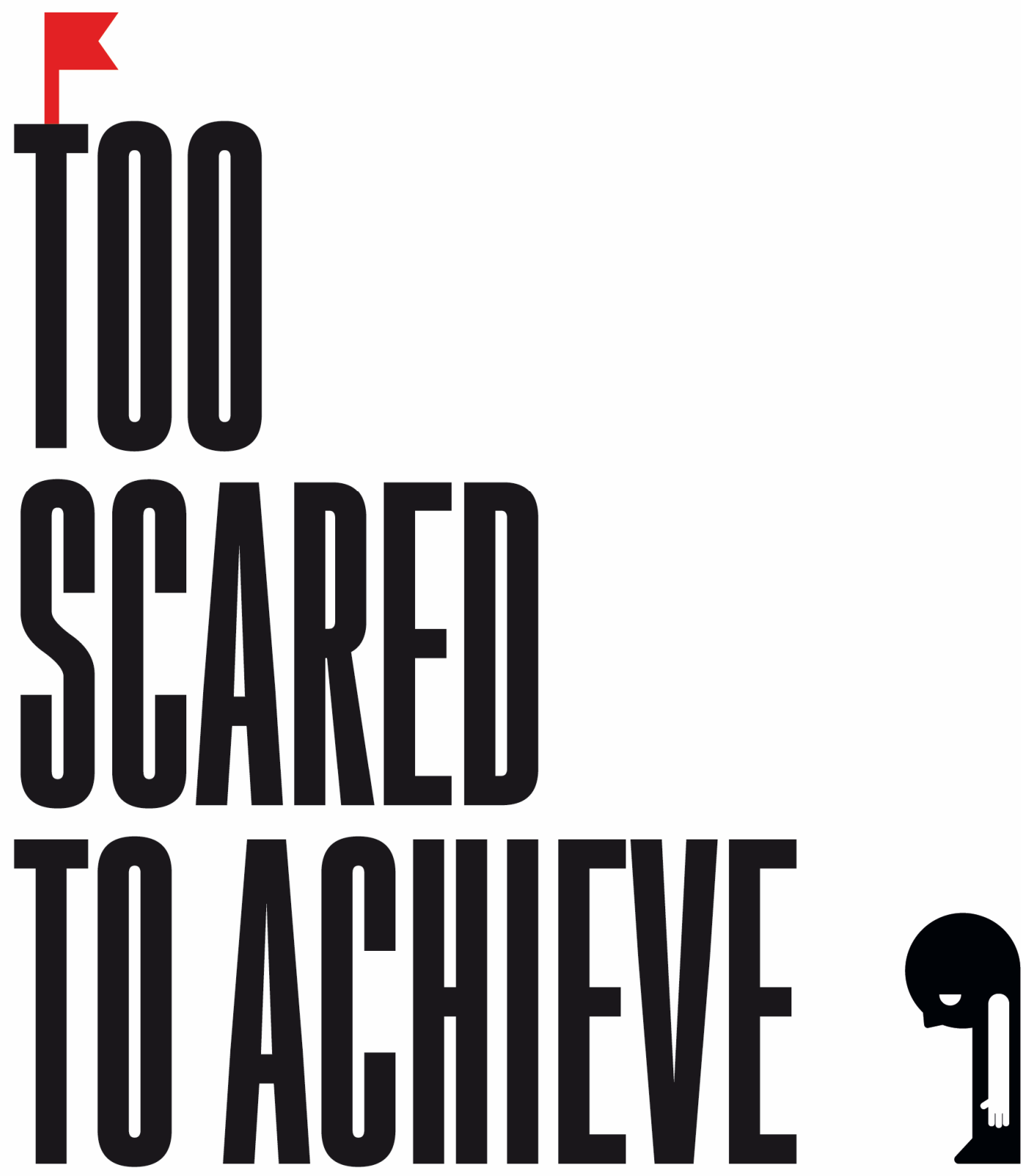

CAROLLINE WEWNER 



\section{TOO SCARED TO ACHIEVE}

THE RELATION BETWEEN NEUROTICISM, CONSCIENTIOUSNESS AND SOCIOECONOMIC OUTCOMES 
(c) Caroline Wehner, 2018

All rights reserved. No part of this publication may be reproduced, stored in a retrieval system or transmitted in any form, or by any means, electronic, mechanical, photocopying, recording, or otherwise, without the prior permission in writing, from the author.

Cover Design: Aryanti Ingenillem

Published by Boekenplan, Maastricht

ISBN: 9789086664498 


\section{TOO SCARED TO ACHIEVE}

\section{THE RELATION BETWEEN NEUROTICISM, CONSCIENTIOUSNESS AND SOCIOECONOMIC OUTCOMES}

\section{DISSERTATION}

to obtain the degree of Doctor at Maastricht University, on the authority of the Rector Magnificus, Prof. Dr. Rianne M. Letschert in accordance with the decision of the Board of Deans to be defended in public on Friday 25th May, 2018, at 12:00 hours

by

Caroline Wehner 


\section{Supervisor:}

Prof. Dr. Lex Borghans

\section{Co-supervisor:}

Dr. Trudie Schils

\section{Assessment Committee:}

Prof. Dr. Didier Fouarge (Chairman)

Dr. Bart Golsteyn

Prof. Dr. Brent W. Roberts (University of Illinois, United States)

Dr. Marion Spengler (University of Tübingen, Germany) 


\section{ACKNOWLEDGEMENTS}

First, I would like to thank my supervisors Trudie Schils and Lex Borghans. They gave me freedom and backup to investigate a research question that highly fascinates me by crossing the border between economics and psychology.

The dissertation largely benefited from many discourses with my colleagues at Maastricht University, the Institute for Labor Economics (IZA) and the Federal Institute for Vocational Education and Training (BIBB) as well as the comments by the assessment committee members Didier Fourage, Bart Golsteyn, Brent Roberts and Marion Spengler and the participants at various international conferences and workshops.

Moreover, I would like to thank my 2012 cohort members - Ayo, Saba, Eva, Tobias, Carla, Juan, Hasse, Stefania, Eriko, Martin, Gintare, Biljana, Tigist, Thuy, Raquel, Ralf and Sheng - and my colleagues from UNU-Merit and the Maastricht Graduate School of Governance who made my time in Maastricht a very positive and exceptional experience.

Last but not least, I am grateful for the continuous encourgagement of my friends and their assistance particularly in the final stage of my dissertation. My special thanks goes to my Markus for all his love, support and care also in tough times as well as to my family who provided me with the foundation to build upon. 



\section{SUMMARY}

Economic studies show that low emotional stability is negatively related to socioeconomic outcomes, while conscientiousness predicts desirable results. Possible mechanisms behind these relations are far less explored. The dissertation addresses this research gap both theoretically and empirically by including psychological insights into an economic model. Differences in expectations and the individual problemsolving ability are investigated as one important channel for the observed relations between personality and socioeconomic outcomes. The results suggest that particularly both low emotionally stable and low conscientious individuals have a higher risk to be trapped in a disadvantageous circle of negative expectations and experiences throughout life. Intervention already in school might reduce this risk. 



\section{CONTENT}

Acknowledgements

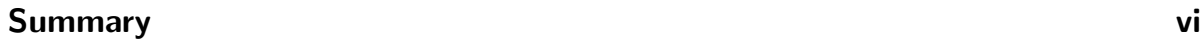

Main Tables xiv

Main Figures $\quad$ xv

1 Introduction 1

1.1 Motivation and Aim . . . . . . . . . . . . . . . . 2

1.2 Approach ...................... 6

2 Theoretical Considerations 9

2.1 Introduction . . . . . . . . . . . . . . . . . . . . . 10

2.2 Psychological Literature . . . . . . . . . . . . . . . . . . 10

2.2.1 Personality Traits . . . . . . . . . . . . . . . . 10

2.2.2 Cognitive Activation Theory of Stress . . . . . . . . . . . . . 13

2.2.3 Concepts of Neuroticism and Conscientiousness . . . . . . . . 14

2.2.4 Concept of Pessimistic Expectations . . . . . . . . . . . . 16

2.3 Economic Model . . . . . . . . . . . . . . . . . . . . . . . . . 18

2.3.1 Personality, Effort and Utility . . . . . . . . . . . . . . 19

2.3.2 Role of Pessimistic Expectations . . . . . . . . . . . . . . . . 24

2.4 Conclusion . . . . . . . . . . . . . . . . . . . . . . . . 29

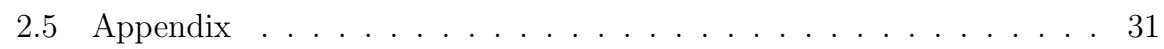

2.5.1 Personality, Effort and Utility . . . . . . . . . . . . 31

2.5.2 Role of Pessimistic Expectations . . . . . . . . . . . . . 37 
3 Personality and Educational Achievement 43

3.1 Introduction . . . . . . . . . . . . . . . . . . . 44

3.2 Data . . . . . . . . . . . . . . . . . . . 45

3.2.1 Educational Achievement . . . . . . . . . . . . . 46

3.2 .2 Personality Traits . . . . . . . . . . . . . . . . . . . 47

3.2 .3 Additional Controls . . . . . . . . . . . . . . . . . . 51

3.3 Results . . . . . . . . . . . . . . . . . . . . 52

3.3.1 Personality and Educational Achievement . . . . . . . . . 52

3.3.2 Personality Change and Educational Achievement . . . . . . . 56

3.4 Conclusion . . . . . . . . . . . . . . . . . . . 58

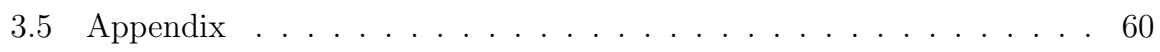

3.5.1 Data Description . . . . . . . . . . . . . . . 60

3.5.2 Robustness Checks . . . . . . . . . . . . . . . . 67

4 Pessimistic Expectations and Educational Achievement 71

4.1 Introduction . . . . . . . . . . . . . . . . . . . 72

4.2 Data . . . . . . . . . . . . . . . . . . 73

4.2.1 Educational Achievement . . . . . . . . . . . . . . . 74

4.2.2 Pessimistic Expectations . . . . . . . . . . . . . . . . . 75

4.2.3 Personality Traits . . . . . . . . . . . . . . . . . . . . . 75

4.2.4 Additional Controls . . . . . . . . . . . . . . . 77

4.3 Results . . . . . . . . . . . . . . . . . . 77

4.3.1 Personality and Pessimistic Expectations . . . . . . . . . . 78

4.3.2 Predictive Power of Pessimistic Expectations . . . . . . . . . . 80

4.4 Conclusion . . . . . . . . . . . . . . . . . . . . . . . . . . 84

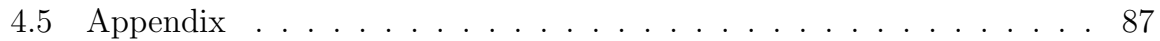

4.5.1 Data Description . . . . . . . . . . . . . 87

4.5.2 Robustness Checks . . . . . . . . . . . . . . . 91

5 Personality and Mental Health $\quad 99$

5.1 Introduction . . . . . . . . . . . . . . . . . . . . . . . 100

5.2 Data . . . . . . . . . . . . . . . . . . . . 101

5.2.1 Mental Health Outcomes . . . . . . . . . . . . . . . . . 102

5.2 .2 Personality Traits . . . . . . . . . . . . . . . . . . 104

5.2 .3 Additional Controls . . . . . . . . . . . . . . 106 
5.3 Results . . . . . . . . . . . . . . . . . . . 106

5.3 .1 Personality and Mental Ill-Health _ . . . . . . . . . 106

5.3.2 Personality Change and Mental Ill-Health . . . . . . . . . . 111

5.4 Conclusion . . . . . . . . . . . . . . . . . . . . . . . . . . . 113

5.5 Appendix . . . . . . . . . . . . . . . . . . . 115

5.5 .1 Data Description . . . . . . . . . . . . . . . . . . 115

5.5 .2 Robustness Checks . . . . . . . . . . . . . . . . . 122

6 Pessimistic Expectations and Mental Health 135

6.1 Introduction . . . . . . . . . . . . . . . . . . . . . . 136

6.2 Data . . . . . . . . . . . . . . . . . . . . . . 137

6.2 .1 Mental Health Outcomes . . . . . . . . . . . . . . . . . . 138

6.2 .2 Pessimistic Expectations . . . . . . . . . . . . . . . 138

6.2 .3 Personality Traits . . . . . . . . . . . . . . . . . . . . . 141

6.2 .4 Additional Controls . . . . . . . . . . . . . . . . . . . 142

6.3 Results . . . . . . . . . . . . . . . . . . . . . . . . . . . 142

6.3.1 Personality Traits and Pessimistic Expectations . . . . . . . 143

6.3.2 Predictive Power of Pessimistic Expectations . . . . . . . . . . 145

6.3.3 Change in Pessimistic Expectations . . . . . . . . . . . . 153

6.4 Conclusion . . . . . . . . . . . . . . . . . . . . 155

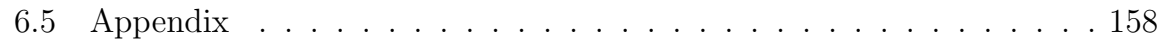

6.5.1 Data Description . . . . . . . . . . . . . . . 158

6.5.2 Robustness Checks . . . . . . . . . . . . . . . . 165

7 Discussion and Conclusion $\quad 185$

7.1 Main Results . . . . . . . . . . . . . . . . . . . . . . . 186

7.2 Discussion of Results . . . . . . . . . . . . . . . . . . . . . 189

7.3 Valorization Addendum . . . . . . . . . . . . . . . . . . 192

$\begin{array}{ll}\text { Bibliography } & 196\end{array}$

$\begin{array}{ll}\text { Curriculum Vitae } & 215\end{array}$ 



\section{MAIN TABLES}

2.1 Big Five Personality Traits . . . . . . . . . . . . . . . . . . . . 11

3.1 Personality Change Between Ages 10 and 16 . . . . . . . . . . . 50

3.2 Role of Emotional Stability and Conscientiousness . . . . . . . . . . . . . 53

3.3 Substitution Effect of Conscientiousness . . . . . . . . . . . . . . . . . 54

3.4 Personality Types and Educational Qualification . . . . . . . . . . . . 55

3.5 Personality Change and Educational Qualification . . . . . . . . . . 57

4.1 Personality and Pessimistic Expectations . . . . . . . . . . . . . . 79

4.2 Role of Pessimistic Expectations . . . . . . . . . . . . . . . . . . . . . . . 80

4.3 Interaction between Personality and Pessimistic Expectations . . . . . . 83

5.1 Correlation of Mental Ill-Health Scores . . . . . . . . . . . . . . . . . . . 104

5.2 Role of Emotional Stability and Conscientiousness . . . . . . . . . . . . . 107

5.3 Substitution Effect of Conscientiousness . . . . . . . . . . . . . 108

5.4 Personality Types and Mental Ill-Health . . . . . . . . . . . . . . . . . 109

5.5 Role of Emotional Stability and Conscientiousness -

Controlling for Mental Ill-Health at Age 16 . . . . . . . . . . . . . . 110

5.6 Personality Types and Mental Ill-Health -

Controlling for Mental Ill-Health at Age 16 . . . . . . . . . . . . . . . 111

5.7 Personality Trait Change and Mental Ill-Health . . . . . . . . . . . . . . 112

6.1 Personality Trait Change and Help- and Hopelessness . . . . . . . . . . . 143

6.2 Helplessness, Personality and Mental Ill-Health . . . . . . . . . . . . . 147

6.3 Hopelessness, Personality and Mental Ill-Health . . . . . . . . . . . . . . 148

6.4 Interaction between Helplessness and Personality . . . . . . . . . . . . . 149

6.5 Interaction between Hopelessness and Personality . . . . . . . . . . . . . 152

6.6 Helplessness Change, Gender and Mental Ill-Health . . . . . . . . . . . . 154 



\section{MAIN FIGURES}

2.1 Sociogenomic Model of Personality Traits . . . . . . . . . . . . . . . . . . 11

2.2 Utility-Maximizing Level of Effort . . . . . . . . . . . . . . . . . . . 21

2.3 Effort Substitution Effect of Conscientiousness . . . . . . . . . . . . . . . 22

2.4 Optimal Effort and Pessimistic Expectations . . . . . . . . . . . . . . 25

2.5 Optimal Effort and Pessimistic Expectations in Relation to Neuroticism and Conscientiousness . . . . . . . . . . . . . . 26

2.6 Effort Substitution Effect between Pessimistic Expectations, Neuroticism and Conscientiousness . . . . . . . . . . . . . . . . . . 28

3.1 Educational Achievement at Age $30 \ldots \ldots$. . . . . . . . . 46

3.2 Personality Traits at Age $10 \ldots \ldots$. . . . . . . . . . . . . . . 49

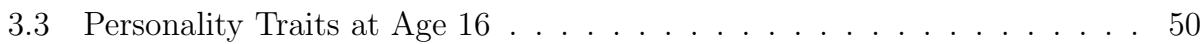

4.1 Educational Achievement at Age $30 \ldots \ldots$. . . . . . . . . . 74

4.2 Exam Expectations at Age $16 \ldots \ldots$. . . . . . . . . . . 76

4.3 Personality Traits at Ages 10 and $16 \ldots \ldots$. . . . . . . . . 77

5.1 Mental Ill-Health at Ages 16, 26, 30, 34 and $42 \ldots$. . . . . . . . . 103

5.2 Personality Traits at Ages 10 and $16 \ldots$. . . . . . . . . . 105

6.1 Mental Ill-Health at Ages 16, 26, 30, 34 and $42 \ldots$. . . . . . . . . . . . 139

6.2 Pessimistic Expectations at Age 16 . . . . . . . . . . . . . . . 140

6.3 Personality Traits at Ages 10 and $16 \ldots$. . . . . . . . . . . 141 

CHAPTER 1

INTRODUCTION 


\subsection{Motivation and Aim}

The personality trait neuroticism ${ }^{1}$ is observed to be mostly negatively related to educational and occupational achievement, financial well-being, marriage or health outcomes, while the personality trait conscientiousness is largely found to be a predictor for socioeconomic success throughout life (Bogg and Roberts, 2004; Roberts et al., 2007; Almlund et al., 2011; Fletcher, 2013; Lundberg, 2013; Dupuy and Galichon, 2014; Mendolia and Walker, 2014a,b; Xu et al., 2015; Egan et al., 2017). However, neuroticism seems to be not inevitably related to a socioeconomic disadvantage and some people concerned are more resilient than others. What drives such differences? To what extent is conscientiousness able to substitute for neuroticism in relation to socioeconomic outcomes by compensating for potentially hindering personality facets? And, what is the role of pessimistic expectations in these associations?

The dissertation aims to go beyond the current economic literature by addressing these questions from both a theoretical and an empirical perspective. First, a theoretical economic framework built on the human capital tradition, but fed with insights from the psychological literature is developed. Second, the hypothesized relationships between neuroticism and socioeconomic outcomes, the substitution effect of conscientiousness and the role of pessimistic expectations are empirically investigated using rich cohort data.

A rapidly growing number of economic studies use personality traits to predict important life outcomes. Personality traits are often measured based on a five-factor personality trait inventory that comprises neuroticism, conscientiousness, agreeableness, extraversion and openness to experience (McCrae and John, 1992; Goldberg, 1993). One possible reason for the observed negative relation between neuroticism and socioeconomic outcomes might be that neurotic individuals have a higher stressreactivity in challenging situations and are more likely to avoid problems rather than solving them due to pessimistic expectations (Connor-Smith and Flachsbart, 2007). On the one hand, this is likely to translate into worse outcomes in various areas of life such as educational achievement. On the other hand, this might result in feelings of sustained stress and mental health problems related to a lower perceived and actual

1 The term "neuroticism" is contrasted by "emotional stability", while "neuroticism", "low emotional stability" and "emotional instability" can be used synonymously. All terms are used in the dissertation. 
problem-solving ability (Penley et al., 2002; Ursin and Eriksen, 2004). Conscientiousness is likely to break the vicious circle of negative expectations and experiences, because conscientious individuals are more likely to apply problem-focused strategies (Connor-Smith and Flachsbart, 2007). However, personality traits are assumed to be rather stable and difficult to change (Roberts, 2009), which makes them relatively inappropriate for intervention.

By contrast, expectations are considered more volatile and adjustable (Roberts, 2009), whereby targeting pessimistic expectations might be a more suitable channel to stop destructive self-fulfilling prophecies. Economic insights into the role of pessimistic expectations in predicting socioeconomic outcomes above and beyond personality might help to further determine high-risk groups and investigate a potential starting point for effective and efficient (pre-)interventions to increase social and economic welfare and to reduce the high non-pecuniary and monetary costs of neuroticism (Cuijpers et al., 2010).

Traditionally, Becker's (1964) human capital theory is the most prominent framework to analyze individual education decisions. ${ }^{2}$ Human capital is defined as the individual knowledge and skills. Schooling is regarded as an investment to acquire these competencies and increase productivity, which results in higher earnings. The individual faces costs of education and a trade-off between current and future income represented by a human capital production function. ${ }^{3}$ The income obtained at the labor market is determined by the acquired human capital. The rational individual makes the education investment decision to maximize the own life cycle utility. Human capital theory usually focuses on formal schooling or post-school training. Nevertheless, the underlying idea can be theoretically transferred to all kinds of education decisions in the private and professional life such as learning how to ride a bike or play the guitar, socializing in school or at parties, searching and applying for a new job or handling a debt burden, because an individual cost-benefit evaluation is likely to be involved at least to some extent. ${ }^{4}$

Education policies subsidize schooling among others due to positive externalities of

2 Cahuc et al. (2014) provide an excellent introduction into human capital theory.

3 Education costs include enrollment fees, purchase of study materials or loss of earnings while studying.

4 For instance, it is less likely that a person is willing to learn to ride a bike when it is unlikely to benefit from the investment, because it is faster to walk due to an unsuitable infrastructure. 


\section{INTRODUCTION}

education $^{5}$ or intergenerational socioeconomic mobility to avoid underinvestment in education related to credit constraints based on market imperfections (Lochner and Monge-Naranjo, 2012). Education expenditures represent a large part of governmental budgets. In 2013, public expenditures in primary to tertiary education constitute on average $4.5 \%$ of the gross domestic product (GDP) in Organisation for Economic Co-operation and Development (OECD) countries (OECD, 2016, pp. 207). However, Carneiro and Heckman (2002) find that only up to $8 \%$ of US adolescents are credit constrained in the short run, whereby their enrollment in post-secondary schooling is impeded. Accordingly, the question arises why some individuals are not willing to attend post-secondary education despite of high expected private returns to education (Cahuc et al., 2014).

Carneiro and Heckman (2002) argue that this rather paradoxical outcome arises due to long-term credit constraints starting very early in life. They emphasize that children's cognitive and non-cognitive abilities as well as their expectations about life chances are mainly shaped by their parents. Indeed, there is evidence that the child's level of emotional stability is both genetically and culturally highly influenced by the family. Neuroticism is considered as heritable to a large extent, although it can also develop based on continuous exposure to anxiety and stress (Roberts, 2009; Cuijpers et al., 2010). There is further evidence that emotional stability is an important factor to explain differences in engagement where learning is necessary and future profits are uncertain (Fouarge et al., 2013; Ryan et al., 2013; Cobb-Clark et al., 2014; Mendolia and Walker, 2014a; Caliendo et al., 2015, 2016). ${ }^{6}$ Accordingly, Heckman, Lochner and Todd (2006) denote the "psychic costs of school" (p. 446) as one reason for the observed phenomenon.

The dissertation takes a deeper glance on these "psychic costs" by theoretically modeling and empirically analyzing potential mechanisms behind the observed relations and by including psychological knowledge into the traditional human capital frame-

\footnotetext{
5 Positive externalities of education include higher tax and social contribution payments, lower crime rates, higher democratic participation and social engagement as well as positive spillover to children (Currie and Moretti, 2003; Szirmai, 2005; Lochner, 2011; Machin, Marie and Vujić, 2011; Machin, Pelkonen and Salvanes, 2011). Cahuc et al. (2014) conclude that social returns even exceed private returns to education.

6 Some of these studies use the psychological concept "locus of control" that refers to the extent to which an individual believes to have control over events in life. Judge and Bono (2001) argue that self-esteem, locus of control and emotional stability are not equal but related psychological concepts.
} 
work. It builds on the economic literature that "extends rational choice [...] models" and "increases the explanatory power of economics by providing it with more realistic psychological foundations" (Wilkinson and Klaes, 2012, p. 3). The assumptions of rational choice models include perfect information, consistent preferences and utility-maximizing behavior. This means that regardless how complex the circumstances are, an individual is assumed to always have full information about the education and labor market choices in the present and the future, can always rank these choices from the worst to the best and will always choose the highest-ranked choice. Accordingly, it neglects individual bounds of rationality related to limited information and future insecurity or heterogeneity in economic preferences and personality traits. Hence, economists have started to address these issues and study social and economic phenomena from a broader perspective by taking into account approaches from disciplines such as psychology, sociology, biology and medicine.

Within economics, two main branches have developed. The first branch uses economic preferences such as risk and time preferences, positive and negative reciprocity, altruism and trust to enrich standard economic models (Dohmen et al., 2012; Wilkinson and Klaes, 2012; Fehr et al., 2013; Falk et al., 2015). The second branch takes advantage of psychological personality concepts such as the fivefactor personality traits (Big-Five), self-esteem or locus of control (Borghans et al., 2008; Almlund et al., 2011) and emphasizes the importance of personality traits for education investments as well as time-variant returns to education (Heckman and Rubinstein, 2001; Heckman, Lochner and Todd, 2006; Heckman, Stixrud and Urzua, 2006). Child development theory that is related to the second branch argues that (pre-)intervention programs aiming to improve the cognitive and non-cognitive competencies of disadvantaged children and their parents relatively early in life are promising in terms of reducing poverty and intergenerational inequalities (Almlund et al., 2011; Björklund and Kjell, 2011).

However, only few studies do provide a coherent framework of possible mechanisms behind the relationship between personality and socioeconomic outcomes (Cunha and Heckman, 2007; Cunha et al., 2010; Ruhm, 2012; Caliendo et al., 2015; Polachek et al., 2015; Caliendo et al., 2016; Rustichini et al., 2016), because the relation between psychological concepts and socioeconomic outcomes is not straightforward and more complicated mechanisms are likely to play a role. Facing this challenge is important, because "a deeper understanding of personality traits promises to 


\section{INTRODUCTION}

enrich economic theory and to understand the sources of, and solutions for, human inequality" (Borghans et al., 2008, p. 85).

Therefore, the dissertation covers a topic that is highly relevant for individuals and society but has been understudied to date. Moreover, it contributes to both the economic literature and the policy debate, because policy-makers still lack information about how to reduce the high non-pecuniary and monetary costs of neuroticism for individuals, employers and society (Cuijpers et al., 2010; Layard et al., 2013).

\subsection{Approach}

The interdisciplinary approach of the dissertation is twofold. On the one hand, the psychological literature is investigated and the insights gained are placed into a theoretical economic framework that (1) focusses on the relationship between neuroticism and socioeconomic outcomes as well as the potential substitution effect of conscientiousness and (2) is extended by the concept of pessimistic expectations (Chapter 2). On the other hand, potential mechanisms regarding the relationship between neuroticism, conscientiousness, pessimistic expectations and socioeconomic outcomes are analyzed empirically (Chapters 3, 4, 5 and 6). Gaining insights into these relations can help to understand heterogeneity in socioeconomic outcomes and identify high-risk individuals with regard to personality characteristics, which might yield important input for successful (pre-)intervention programs.

As representatives for socioeconomic outcomes, educational achievement and mental health are used. These outcomes are chosen for two reasons: On the one hand, analyzing educational achievement and mental health is an economically relevant starting point, because both domains are important for individual socioeconomic success throughout life and potential problems have their onset in childhood or adolescence (Peracchi, 2006; OECD, 2011; Oreopoulos and Salvanes, 2011; Tefft, 2012; Helliwell et al., 2013; Layard et al., 2013; Bratti and Mendola, 2014; Anderson et al., 2015; Cornaglia et al., 2015; Bubonya et al., 2017). On the other hand, educational achievement is an objective measure, while mental health is a subjective one. If the hypothesized relationships exist, variation in both outcomes related to personality traits should go hand in hand.

Chapters 3 and 4 focus on educational achievement. Chapter 3 investigates the predictive power of (1) low emotional stability at the age of 16 for educational achieve- 
ment at the age of 30 as well as the possible substitution effect of conscientiousness and (2) a change in emotional stability and conscientiousness between the ages of 10 and 16 for educational achievement at the age of 30. Chapter 4 analyzes the potential channel of pessimistic expectations for the relation between personality and educational achievement by testing the association (1) between emotional stability as well as conscientiousness at the ages of 10 and 16 and pessimistic expectations at the age of 16 and (2) between emotional stability, conscientiousness as well as pessimistic expectations at the age of 16 and educational achievement at the age of 30 .

Chapters 5 and 6 focus on mental health. Chapter 5 analyzes the predictive power of (1) low emotional stability at the age of 16 for mental health problems at the ages of $16,26,30,34$ and 42 as well as the possible substitution effect of conscientiousness and (2) a change in emotional stability and conscientiousness between the ages of 10 and 16 and mental ill-health in adolescence and adult age. Chapter 6 investigates the relation (1) between the personality traits emotional stability and conscientiousness at the age of 10 and 16 and pessimistic expectations at the age of 16, (2) between emotional stability, conscientiousness as well as pessimistic expectations at the age of 16 and mental ill-health in adolescence and adult age and (3) between a change in pessimistic expectations between the ages of 16 and 30 and mental health problems at later ages.

The analyses in the dissertation are based on data from the 1970 British Cohort Study (BCS70). The data is deposited at the UK Data Archive by the Centre for Longitudinal Studies at the Institute of Education, University of London. BCS70 is funded by the Economic and Social Research Council (ESRC) and allows following people from birth up until the age of 42 , with rich information about personality traits, pessimistic expectations, cognitive ability, socioeconomic background, educational achievement and mental health.

The dissertation concludes in Chapter 7 with a summary and discussion of the main results as well as a provision of policy implications and an introduction into existing (pre-)intervention programs. 



\section{CHAPTER 2}

\section{THEORETICAL CONSIDERATIONS*}




\section{$2.1 \quad$ Introduction}

What are potential mechanisms behind the relationship between neuroticism and socioeconomic outcomes? To what extent is conscientiousness able to substitute for neuroticism in relation to socioeconomic outcomes? And, what is the role of pessimistic expectations in these associations? To answer these questions, knowledge from psychology and economics is combined into a theoretical framework. The term "education investment" is thereby understood as the decision to tackle challenges and acquire new skills. This includes school- and career-related education situations, but also challenges in the private field without official testing.

First, insights from psychology are provided and discussed. Second, an economic framework of neuroticism, conscientiousness and pessimistic expectations is presented. The starting point is a basic model assuming no other differences between individuals except their level of neuroticism and conscientiousness, yet in a more advanced model the assumptions are relaxed by allowing for differences in the individuals' level of pessimistic expectations. In order to make the line of argumentation more comprehensible, a practical example of teenagers who have to face an exam at school and who differ in their exam preparation is provided. ${ }^{7}$

\section{$2.2 \quad$ Psychological Literature}

\subsubsection{Personality Traits}

Different personality theories exist. Roberts (2009) provides an introduction to personality theory and argues that "traits are made up of stable, enduring patterns of states (thoughts, feelings and behaviors) and thus cause future states. [...] It is the repetitions in states that capture what we mean by a trait. [...] The differentiation of state from trait and the proposal that environments affect states directly also helps to explain shifts in behaviors that may have little or nothing to do with changes in personality traits." (pp. 7, Figure 2.1). ${ }^{8}$

\footnotetext{
7 For the sake of simplicity, the masculine form is used throughout the chapter. Nevertheless, the economic model should be taken to refer equally to both males and females.

8 Alternative relationships shown in Figure 2.1 are discussed later in this chapter.
} 
Figure 2.1: Sociogenomic Model of Personality Traits

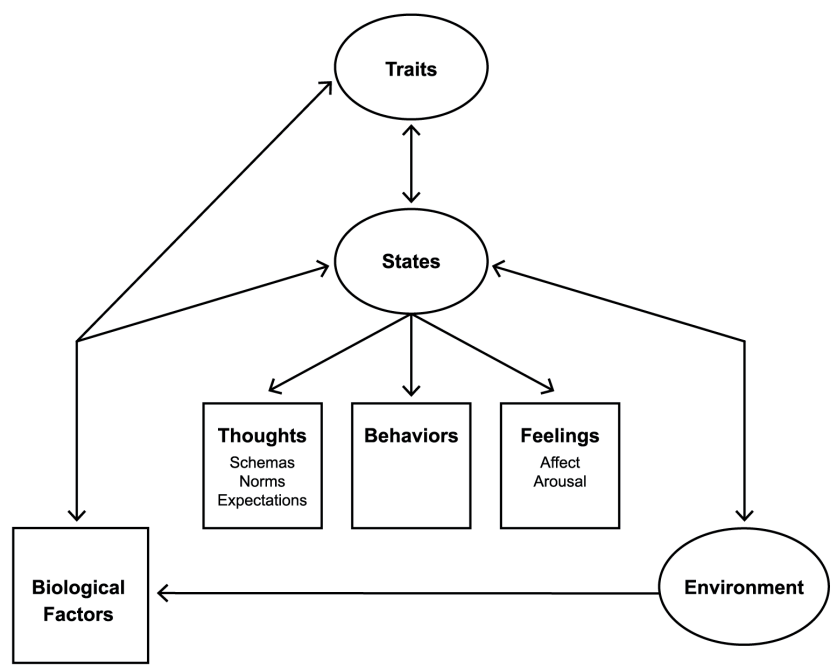

Source: Roberts (2009).

Table 2.1: Big Five Personality Traits

\begin{tabular}{|c|c|c|}
\hline Personality Trait & The degree to which a person... & Lower-Order Facets \\
\hline Neuroticism & $\begin{array}{l}\text {... experiences the world as threat- } \\
\text { ening. }\end{array}$ & $\begin{array}{l}\text { anxiety, angry hostility, depression, } \\
\text { self-consciousness, impulsiveness, } \\
\text { vulnerability }\end{array}$ \\
\hline Conscientiousness & $\begin{array}{l}\ldots \text { is willing to comply with con- } \\
\text { ventional rules. }\end{array}$ & $\begin{array}{l}\text { competence, order, dutifulness, } \\
\text { achievement, striving, self-discipline, } \\
\text { deliberation }\end{array}$ \\
\hline Extraversion & $\begin{array}{l}\ldots \text { needs attention and social inter- } \\
\text { action. }\end{array}$ & $\begin{array}{l}\text { warmth, gregariousness, assertive- } \\
\text { ness, activity, excitement-seeking, } \\
\text { positive emotions }\end{array}$ \\
\hline Agreeableness & $\begin{array}{l}\text {... needs pleasant and harmonious } \\
\text { relations with others. }\end{array}$ & $\begin{array}{l}\text { trust, straightforwardness, altru- } \\
\text { ism, compliance, modesty, tender- } \\
\text { mindedness }\end{array}$ \\
\hline Openness & ... needs intellectual stimulation. & $\begin{array}{l}\text { fantasy, aesthetics, feelings, actions, } \\
\text { ideas, values }\end{array}$ \\
\hline
\end{tabular}

Source: Hogan and Hogan (2007), p. 7 and Matthews et al. (2009), p. 25. 
The empirical analysis of personality traits started with Allport and Odbert (1936) who extracted five dimensions of personality out of 17,953 personality-related words based on a lexical approach. The most prominent system to measure personality traits is the five-factor model known as Big Five model (Matthews et al., 2009). The five personality dimensions are neuroticism or emotional stability, consciousness, agreeableness, extraversion and openness presented in Table 2.1.

Irrespective of the broad acceptance and application, the model is not without criticism: (1) The appropriate number of factors remains controversially discussed. ${ }^{9}$ (2) The model is derived from factor analysis and not explained by a basic theory. (3) Disagreement exists about the classification of lower-order facets into higher trait dimensions (Borghans et al., 2008). However, for the purpose of the dissertation the five-factor model is believed to be sufficiently tested and validated.

Most scholars believe that personality traits are to a certain extent determined by genes and develop in childhood and adolescence, but are rather mature and stable in adulthood (Robins et al., 2001; Cuijpers et al., 2010; Eisenberg et al., 2014). However, while Costa and McCrae (1994) argue that personality is "set like a plaster" by the age of 30 , others find that personality traits might change in adult age (Roberts, 1997; Scollon and Diener, 2006; van Aken et al., 2006; Lüdtke et al., 2011). The variation in findings might be explained by the type of personality change assessed (Robins et al., 2001). ${ }^{10}$ Additionally, the intensity of environmental stimuli are likely to play a role (Robins et al., 2001). In sum, one might conclude that personality traits are rank-order stable in adulthood (Roberts and Del Vecchio, 2000).

Economists who consider personality traits in their research can use valid measurement tools provided by psychology. Reasons for the omission of personality traits from most economic studies are a "lack of familiarity of economists with these personality measures" and the necessity "that many economists have yet to be convinced of their predictive validity, stability, or their causal status" (Borghans et al., 2008, p. 975).

\footnotetext{
9 See Cattell et al. (1970) and Eysenck (1992) who promote a 16 or three factor systematic.

10 For instance, Roberts et al. (2006) and Cobb-Clark and Schurer (2012) address mean level personality changes in the population that refer to the change in average personality trait levels over time. Roberts and Del Vecchio (2000) analyze rank-order stability of personality that refers to the correlation of personality scores between two points in time. Roberts (1997) investigates individual changes in personality traits.
} 


\subsubsection{Cognitive Activation Theory of Stress}

To gain deeper insights into possible mechanisms behind the relationship between personality and socioeconomic outcomes, Ursin and Eriksen (2004)'s Cognitive Activation Theory of Stress is useful. It argues that individuals react with arousal when they are confronted with a stimulus. A stimulus is defined as a discrepancy between what the individual expects and what happens in reality. The announcement of an exam in school might be considered as a negative and threatening stimulus. Individuals "report this as stress" (Ursin and Eriksen, 2004, p. 571), whereby the extent of stress depends on the individual's stimulus expectancies, which are based on previous experiences and learning. "When the subject has learned that one stimulus $[\ldots]$ predicts the occurrence of another event [...] this is referred to as stimulus expectancy" (Ursin and Eriksen, 2004, p. 573). For instance, holding all other factors constant, a teenager whose parents usually provided constructive feedback including in the event of failure - probably perceives the sudden announcement of an exam as less threatening than someone who experienced excessive parental punishment after previous failure.

The stress response is uncomfortable but not per se problematic regarding education or mental health outcomes. It is much more a "safety system, which guarantees priority to serious and sudden discrepancies" (Ursin and Eriksen, 2004, p. 573). Phasic stress is vitally important to find proper solutions in challenging situations, i.e. it might initiate a proper exam preparation. Nonetheless, feelings of stress persist until the problem is at least mentally solved. "Sustained arousal may lead to pathology" (Ursin and Eriksen, 2004, p. 570).

Whether the individual's arousal in the challenging exam situation causes harmless phasic or damaging sustained stress depends on the individual response outcome expectancy, which is also based on previous experiences and learning. "When the subject has learned that performance of a response [...] brings a certain outcome [...] this is referred to as response outcome expectancy" (Ursin and Eriksen, 2004, p. 573). Response outcome expectancies are defined as positive (coping), negative or uncertain. Coping means that the teenager has established the expectancy to handle the exam with a positive result. More generally, coping is defined as "problem-solving thoughts and actions" (Penley et al., 2002, p. 552). Coping leads to harmless, phasic stress. An uncertain or negative outcome expectancy is much more problematic. An 
uncertain response outcome expectancy means that the teenager cannot recognize a relationship between the response and the outcome, whereby he perceives having no control over the exam's result. By contrast, a negative response outcome expectancy reflects the opposite of coping, i.e. the teenager expects exam failure whatever he does and considers this as his fault, which is related to a feeling of guilt. Both uncertain and negative response outcome expectancies are related to harmful sustained stress because the perceived problem-solving ability is reduced and the challenge mentally persists.

\subsubsection{Concepts of Neuroticism and Conscientiousness}

Individuals who score high in neuroticism are defined as "worrying, nervous, emotional insecure" (Cervone and Pervin, 2014, p. 265). Neuroticism is closely related to low self-esteem, external locus of control and low generalized self-efficiency (Judge et al., 2002). For instance, Eyseneck (1990) defines self-esteem as a lower order indicator of neuroticism and Judge et al. (2002) propose using a common core construct for the four psychological concepts. Following these personality psychologists and simplifying the model, neuroticism is considered representative of the other concepts.

Referring to Ursin and Eriksen (2004)'s Cognitive Activation Theory of Stress, it is likely that individual stimulus and response outcome expectancies vary with personality traits. Low emotionally stable individuals are prone to anxiety, chronic worry and distress (Cervone and Pervin, 2014, p. 265). This suggests that low emotionally stable individuals are more likely to have both negative and threatening stimulus as well as negative or uncertain response outcome expectancies. In other words, low emotionally stable teenagers are likely to perceive more exams in school as threatening and to perceive an exam as more threatening compared with more emotionally stable teenagers. Accordingly, they are more likely to have a reduced perceived problem-solving ability, which is related to uncomfortable sustained stress and boosts the risk of mental health problems.

Furthermore, low emotional stability is associated with emotion-focused (e.g., avoidance) and disengagement coping (Connor-Smith and Flachsbart, 2007). "Personality may affect coping strategy selection directly, by constraining or facilitating use of specific strategies, or indirectly, by influencing the nature and severity of stressors 
experienced [...] the threat sensitivity underlying [neuroticism] may trigger disengagement [...] [Neuroticism] is associated with high rates of stress exposure and intense emotional and physiological reactivity to stress" (Connor-Smith and Flachsbart, 2007, p. 1081).

Emotion-focused and disengagement coping might reduce these negative emotions and produce relief, which can be considered a short-run gain. However, in this way destructive expectations are likely to translate into a reduced actual problem-solving ability, because procrastination or avoidance is likely to sustain the underlying challenge. For instance, someone who procrastinates or avoids the exam preparation in order to distract from the uncomfortable exam is more likely to have a worse exam result or repeat the exam.

Even worse, a vicious circle based on negative expectations and negative experiences is completed and likely to be sustained. "Although [neuroticism] may facilitate disengagement, and make it beneficial in the short-term, these immediate benefits for vulnerable individuals are likely outweighed by long-run costs" (Connor-Smith and Flachsbart, 2007, p. 1102). Furthermore, "short-term, personality-related benefits of disengagement for [neurotic] individuals may amplify the direct effect of [neuroticism] on the tendency to disengage, explaining why [neurotic] individuals continue to use strategies that produce poor long-term results" (Connor-Smith and Flachsbart, 2007, p. 1082).

Roberts (2009) argues that states of thoughts (expectancies), feelings (arousal) and behaviors (avoidance or effort) are to some extent triggered by personality traits in an unconscious, repetitive manner. Under the assumption that individuals are confronted with various smaller and larger challenges day-by-day (e.g., in school or at work as well as in social relations), situations where feelings of sustained stress related to destructive thoughts and avoidance behavior may occur are likely to cumulate particularly for less emotionally stable individuals. This might be associated with mental health problems, worse educational attainment and a general socioeconomic disadvantage in the long run. This line of reasoning is consistent with the psychology literature. Based on a meta-analytic review, Penley et al. (2002) show that avoidance coping is negatively correlated with mental health outcomes. ${ }^{11}$ They provide several explanations for the negative relation between avoidance coping and

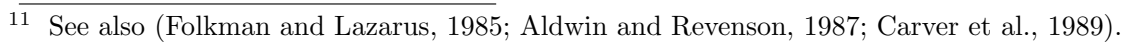


psychological health outcomes, including a further rise in anxiety and worsened outcomes, an impairment of social relations and an increase in unhealthy behaviors such as taking drugs or alcohol.

By contrast, conscientious individuals are more likely to apply problem-focused and engagement coping strategies (Connor-Smith and Flachsbart, 2007) and are considered as organized, persistent and ambitious (Cervone and Pervin, 2014). Referring to a situation in school, Trautwein et al. (2015) show that this is even the case when the individual is less interested in a specific subject. Accordingly, effective coping is more likely in situations of unavoidable, but influenceable challenges. Therefore, higher conscientiousness might be able to substitute for lower emotional stability, whereby less emotionally stable but more conscientious individuals are more likely to have improved education outcomes and are less likely to experience sustained stress and mental health issues compared with low emotionally stable and low conscientious individuals.

\subsubsection{Concept of Pessimistic Expectations}

A distinction is often made between traits and states (Steyer et al., 1999; Nezlek, 2007). Whereas traits - including personality traits such as neuroticism - could be regarded as more stable or more difficult to change in the short run, states including thoughts and pessimistic expectations - are presumed to be more volatile and change across situations. Roberts (2009) argues that thoughts, feelings and behavior are influenced by the individual personality, environmental conditions and biological factors (Figure 2.1). Projecting this idea on the relation between emotional stability, pessimistic expectations and social conditions, some related psychological concepts are discussed.

First, the social and economic environment is likely to influence states. Ursin and Eriksen (2004)'s idea of individual stimulus and response outcomes expectancies is related to cognitive vulnerability stress models that denote pessimistic expectations as negative cognitive style as well as negative attributional or inferential style (e.g., Hankin and Abramson, 2001, 2002; Mezulis et al., 2006; Hyde et al., 2008). Negative cognitive style is based on previous experiences and learning. It refers to the explanation of negative life events as being stable (versus unstable), global (versus specific) and internal (versus external) (Abramson et al., 1978). These models demonstrate a 
direct relationship between negative life events in childhood and negative cognitive style at older age. Mezulis et al. (2006) state that "repeated or chronic negative life events in childhood may directly contribute to the development of a more negative cognitive style as children, over time, internalize the belief that negative events are stable, have negative consequences, and are attributable to aspects of themselves." (p.1013). Earlier formulated concepts include the Learned Helplessness Theory introduced by Overmier and Seligman (1967), Helplessness Theory by Seligman (1975), the Reformulated Learned Helplessness Theory by Abramson et al. (1978) and the Hopelessness Theory by Abramson et al. (1989).

Psychological studies further suggest that the relationship between negative life events in childhood and the likelihood of developing a tendency for pessimistic thoughts is moderated by parenting style. Mezulis et al. (2006) argue that pessimistic thoughts are related to "more negative [parental] feedback about the causes and consequences of stressful events" (p. 1013), as well as "parental restrictiveness, rejection, low affection, and perfectionist expectations in childhood" (p. 1014). As an example, consider a situation of failure in a math exam in school. A teenager who has developed a negative cognitive style, i.e. a general tendency to have pessimistic expectations, is more likely to expect failure in subsequent math exams (stable) as well as in other disciplines with negative consequences in the future (global), whereby he will consider the experience of failure as a proof for his incompetence (internal). These pessimistic considerations are likely to be related to disengagement in the future (Connor-Smith and Flachsbart, 2007; Dunbar et al., 2013), whereby he is likely to devote less effort to the preparation of future exams because he believes that he will fail regardless.

Second, the relationship between negative life events in childhood and the likelihood of developing a tendency for pessimistic thoughts is moderated by personality. Children who score low in emotional stability have a higher risk of developing pessimistic thought patterns when experiencing negative life events. Mezulis et al. (2006) state that "children high in withdrawal negativity typically dislike or avoid novel situations; show distress to novelty; become upset, fearful, sad, or tearful easily; and appear highly sensitive to negative stimuli (Belsky et al., 1996)" (p. 1013). This suggests that pessimistic expectations in challenging situations are likely to be related to future outcomes which is analyzed in the dissertation. However, controlling for the source, number and severity of negative life events related to pessimistic 
expectations would exceed the scope of the dissertation.

Third, states are likely to influence personality in the long run. Neuroticism is considered as $50 \%$ to $60 \%$ heritable, but can also develop based on continuous exposure to anxiety and stress (Roberts, 2009; Cuijpers et al., 2010). Roberts (2009) states that "continuous states of anxiety and stress can lead to neuroanatomical changes in brain structures (McEwen et al., 2006). Stressful states likely interact with genes responsive to stress, which, in turn, affect the neuroanatomy that shapes the habitual ability of the person to respond to future environmental insults - thus, a trait is born." Furthermore, there is evidence that making positive experience in life can also change personality in the long-run (Scollon and Diener, 2006; van Aken et al., 2006; Lüdtke et al., 2011). This means that personality traits are no longer considered as being fixed by psychologists. Therefore, the role of a personality trait change between the ages of 10 and 16 for socioeconomic outcomes at older age is studied. However, analyzing the personality change itself is not the core of the dissertation.

\subsection{Economic Model}

Putting these insights from the psychological literature into economic terms, the individual aims to solve a challenging situation with a utility-maximizing level of effort determined by the individual's expected benefits and costs related to effort. It is considered that educational achievement and mental health problems are influenced by the individual's personality, which affects the individual perceived and actual problem-solving ability. The focus lies on neuroticism and conscientiousness. Subsequently, the role of pessimistic beliefs and expectations is introduced.

To illustrate the model, an example of teenagers facing an exam at school is used. It is assumed that the exam result fully depends on the individual's effort related to the exam preparation. Effort can be the time that the person devotes to exam preparation, although it could also represent the intensity with which someone prepares (i.e. concentration). The model refrains from any teacher effects and assumes that the teenagers have equal cognitive ability, live in an equal social environment and have equal biological characteristics. Therefore, they only differ with respect to the personality traits neuroticism and conscientiousness. 


\subsubsection{Personality, Effort and Utility}

Following human capital theory, it is assumed that every teenager makes his effort decision to maximize his own utility ( $U$, such as exam grade or happiness) based on the expected benefits $(B)$ and the costs of effort $(C)$ :

$$
\operatorname{Max} U=B-C
$$

Benefits are the returns to effort, i.e. the grade on the exam or other factors such as parental or labor market recognition. Costs are the costs of effort, i.e. time and energy devoted to the exam preparation. However, as discussed above, expected benefits $(B)$ are likely to be influenced by personality. Therefore, the utility function can be described such that:

$$
U(f, n, c)=c F(f)+(1-c)(1-n) F(f)-\beta f
$$

The first part of the equation $c F(f)+(1-c)(1-n) F(f)$ relates to benefits of effort, while $\beta f$ equals the costs of effort. Benefits of effort are related to a production function which comprises two elements: a routine component $(c F(f))$ and a non-routine component $((1-c)(1-n) F(f))$. The routine component is related to universally relevant skills such as an appropriate work organization and time management as well as the ambition and self-discipline to realize own plans. These skills are likely to increase the translation of effort into utility and they are related to conscientiousness $(c, 0<c<1)$. Thus, the higher conscientiousness, the higher the probability that the individual establishes and refines these skills. Related to the example of the exam preparation, this means that a more conscientious teenager is more likely to have his learning material completed and organized, is more likely to have a schedule prepared that splits the learning content into smaller and better digestible pieces, is more likely to stick to his learning schedule and thus, is more likely to have sufficient time to clarify open questions. Therefore, the routine-based component $(c F(f))$ is related to a gain of control over the challenge's outcome and a decrease of arousal in the challenging situation, whereby the translation of effort into utility is not hindered by negative or distracting emotions and cognition.

However, another part of the challenge is related to entering new territory, whereby a reliance on preparation is impossible. The non-routine component $((1-c)(1-$ 
$n) F(f))$ involves two types of production losses. First, the production loss increases with the non-routine character of the task, which is negatively related to conscientiousness $(1-c)$. The psychological literature shows that conscientiousness is related to attitudes such as self-discipline, deliberation and problem-focused coping. Those general skills are likely to be valuable also in non-routine situations. The conscientious teenager is more likely to deliberately consider possible problem-solving strategies, courageously tackle the non-routine task and successfully solve it. Second, the production loss increases with neuroticism $(1-n, 0<n<1)$. The psychological literature argues that the level of neuroticism affects the extent to which the person considers the exam as a challenge. Considering that the exam is decisive for the teenager's future, it can potentially be threatening and might work as a stress stimulus. A person with a higher level of neuroticism is probably more stressed about living up to the teacher's expectations and is expected to experience a higher level of arousal. This is leaving him with an uneasy feeling and the neurotic teenager is likely to devote effort to eliminate this uncomfortable arousal (i.e. reduced concentration or avoidance). This is expected to lower his grade compared with someone who is less neurotic.

Finally, the cost of effort are related to a scaling factor $(\beta)$ times the effort $(f)$ devoted. $\beta$ is assumed to be related to the opportunity costs of effort. Figure 2.2 shows the individual's benefits and costs of effort related to utility. The benefit function is positive and upward sloping, albeit at a diminishing rate or concave. Accordingly, utility increases with effort, although the effect of extra effort decreases when effort increases. The cost function is assumed to be linear and increasing with effort.

The individual aims to maximize own utility and can choose an effort level to achieve this. Given that $F(f)=f^{\alpha}$ with $0<\alpha<1$, the utility function can be described such that:

$$
U(f, n, c)=c f^{\alpha}+(1-c)(1-n) f^{\alpha}-\beta f
$$

The optimal level of effort $f^{*}$ is found by solving the first-order condition with 
Figure 2.2: Utility-Maximizing Level of Effort

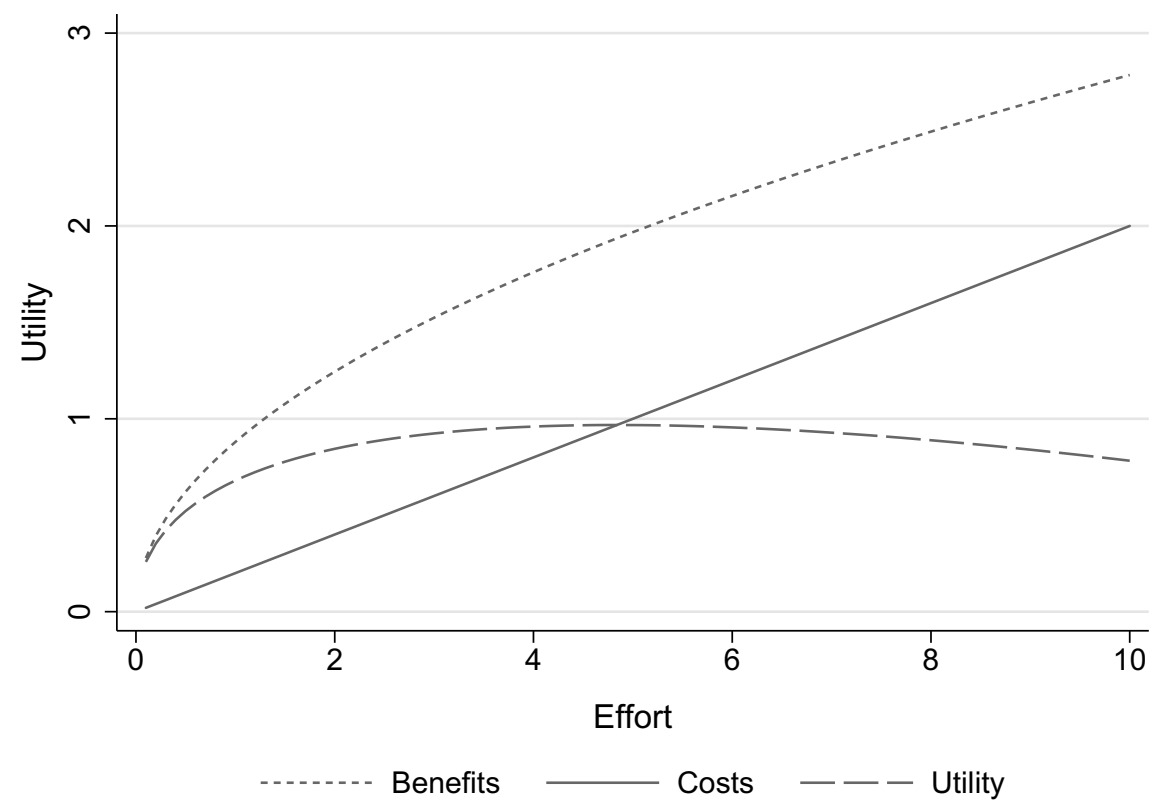

Note: Used parameters are $\alpha=0.5, \beta=0.2, c=0.4$ and $n=0.2$.

respect to effort:

$$
\begin{aligned}
\frac{\partial U}{\partial f} & =\alpha c f^{\alpha-1}+\alpha(1-c)(1-n) f^{\alpha-1}-\beta=0 \\
f^{*} & =\left(\frac{\beta}{\alpha(1-n+c n)}\right)^{\frac{1}{\alpha-1}}
\end{aligned}
$$

The individual's optimal effort level is affected by personality, here neuroticism $(n)$ and conscientiousness $(c) . f^{*}$ can be written as a function of $n$ and $c: f^{*}=f^{*}(n, c)$. In order to derive whether optimal effort $f^{*}$ increases or decreases in response to a change in neuroticism, or alternatively, conscientiousness, the first-order partial derivative of optimal effort regarding neuroticism, or alternatively, conscientiousness is calculated. Taking the partial derivative of Equation 2.2 with respect to $n$ yields:

$$
\frac{\partial f^{*}}{\partial n}=\frac{1}{\alpha-1} \frac{\beta^{\frac{1}{\alpha-1}} \alpha(1-c)}{(\alpha(1-n+c n))^{\frac{\alpha}{\alpha-1}}}<0
$$


Figure 2.3: Effort Substitution Effect of Conscientiousness

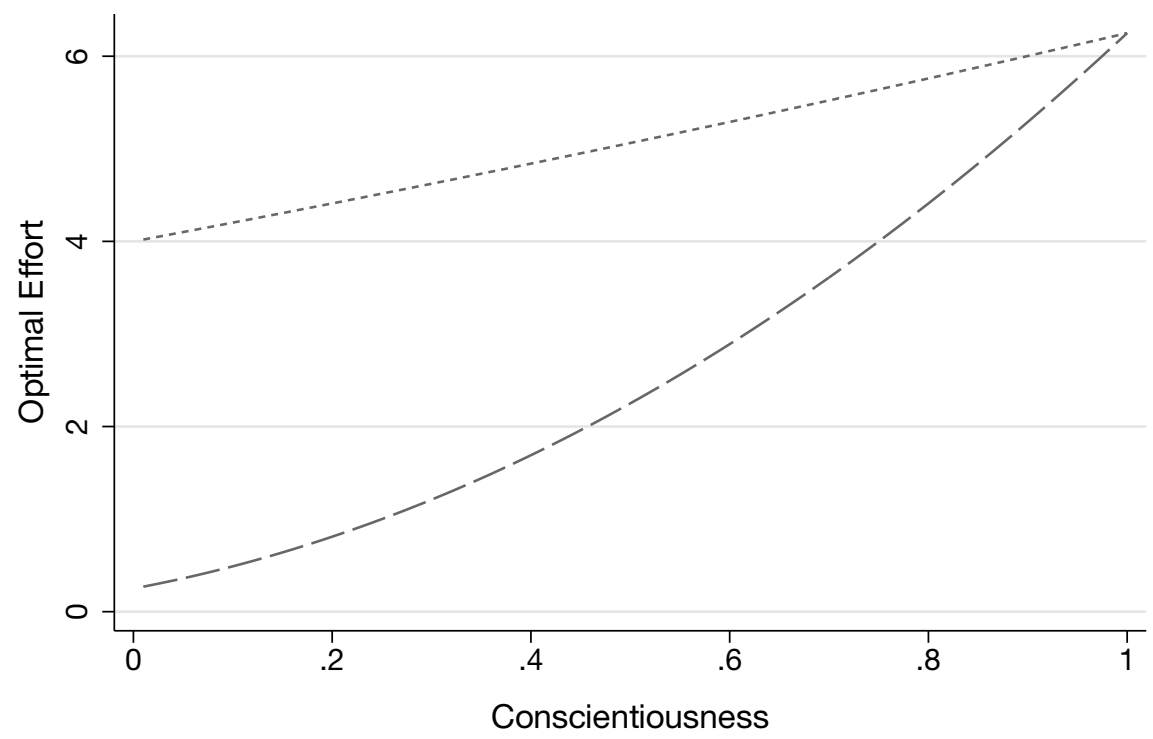

Neuroticism $=0.2---$ Neuroticism $=0.8$

Note: Used parameters are $\alpha=0.5$ and $\beta=0.2$.

Since $0<\alpha<1,0<\beta<1$ and $0<c<1$, it follows that the first term is negative and the second term is positive. Thus, $f_{n}^{*}<0$ and optimal effort decreases with neuroticism. Taking the partial derivative of Equation 2.2 with respect to $c$ yields:

$$
\frac{\partial f^{*}}{\partial c}=\frac{1}{\alpha-1} \frac{-\beta^{\frac{1}{\alpha-1}} \alpha n}{(\alpha(1-n+c n))^{\frac{\alpha}{\alpha-1}}}>0
$$

Since $0<\alpha<1,0<\beta<1$ and $0<c<1$, both the first and second terms are negative (nominator is negative and denominator is positive). Thus, $f_{c}^{*}>0$ and optimal effort increases with conscientiousness.

Further, the substitution effect of conscientiousness $(c)$ on optimal effort related to neuroticism $f_{n}^{*}$ can be derived:

$$
\frac{\partial^{2} f^{*}}{\partial n \partial c}=-\alpha \beta^{\frac{1}{\alpha-1}}\left(\frac{\alpha^{2}-\alpha(1-n+c n)}{(\alpha-1)^{2}(\alpha(1-n+c n))^{\frac{\alpha}{\alpha-1}+1}}\right)>0
$$


Given that both terms are negative, it is shown that the substitution effect of $c$ on optimal effort $f_{n}^{*}$ is positive $\left(f_{n c}^{*}>0\right)$. This means that the effect of neuroticism differs depending on the level of conscientiousness and that at any level of neuroticism, optimal effort is higher with increasing conscientiousness. Figure 2.3 shows that optimal effort increases stronger with an increase in conscientiousness for someone who is highly neurotic compared to someone who is less neurotic. However, this effect is smaller when the level of conscientiousness is low.

Next, the relation between optimal utility, neuroticism and conscientiousness is derived. Optimal utility $\left(U^{*}\right)$ can be written as:

$$
U^{*}\left(f^{*}, n, c\right)=f^{\alpha}(1-n+c n)-\beta f^{*}
$$

Optimal effort is:

$$
f^{*}=\beta^{\frac{1}{\alpha-1}}(\alpha(1-n+c n))^{\frac{1}{1-\alpha}}
$$

Substituting optimal effort $\left(f^{*}\right)$ into optimal utility $\left(U^{*}\right)$ yields:

$$
\begin{aligned}
U^{*}\left(f^{*}, n, c\right) & =\left(\beta^{\frac{1}{\alpha-1}}(\alpha(1-n+c n))^{\frac{1}{1-\alpha}}\right)^{\alpha}(1-n+c n) \\
& -\beta\left(\beta^{\frac{1}{\alpha-1}}(\alpha(1-n+c n))^{\frac{1}{1-\alpha}}\right)
\end{aligned}
$$

Taking the partial derivative of Equation 2.3 with respect to $n$ yields:

$$
\frac{\partial U^{*}}{\partial n}=-\beta^{\frac{\alpha}{\alpha-1}}(1-c)(\alpha(1-n+c n))^{\frac{\alpha}{1-\alpha}}<0
$$

Given that $-\beta^{\frac{\alpha}{\alpha-1}}<0,1-c>0$ and $\left.\alpha(1-n+c n)\right)^{\frac{\alpha}{1-\alpha}}>0$, it follows that $U_{n}^{*}<0$. Optimal utility decreases with neuroticism. Based on the psychological literature, it can be expected that neurotic teenagers are more likely to suffer from sustained stress and procrastinate or even avoid the challenging exam preparation. Thus, general discomfort is likely and the exam grade achieved is probably lower compared to the teenager who actively tackles the challenge. In sum, this scenario is related to a reduced utility for the neurotic individual. 
Taking the partial derivative of Equation 2.3 with respect to $c$ yields:

$$
\frac{\partial U^{*}}{\partial c}=n \beta^{\frac{\alpha}{\alpha-1}}(\alpha(1-n+c n))^{\frac{\alpha}{1-\alpha}}>0
$$

Given that $n \beta^{\frac{\alpha}{\alpha-1}}>0$ and $\alpha(1-n+c n)^{\frac{\alpha}{1-\alpha}}>0$, it follows that $U_{c}^{*}>0$. Optimal utility increases with conscientiousness. Conscientious individuals are by definition organized, ambitious and self-disciplined and more likely to use problem-focused coping, whereby the conscientious teenager is probably better prepared, less aroused and receives a better grade in the exam. Moreover, conscientiousness can potentially mitigate the negative relationship between neuroticism and optimal utility. To observe this, $U_{n c}^{*}$ is derived, which yields:

$$
\frac{\partial^{2} U^{*}}{\partial n \partial c}=\beta^{\frac{\alpha}{\alpha-1}}\left(\frac{1-\alpha-n+c n}{1-\alpha-n+c n+\alpha n-\alpha c n}(\alpha(1-n+c n))^{\frac{\alpha}{1-\alpha}}\right)>0
$$

Given that $\beta^{\frac{\alpha}{\alpha-1}}>0$ and $(\alpha(1-n+c n))^{\frac{\alpha}{1-\alpha}}>0, U_{n c}^{*}>0$. This substitution effect is generally positive. However, for very high levels of neuroticism in relation to conscientiousness, or when $n>\frac{1-\alpha}{1-c}$, the substitution effect is negative. This means that for extremely neurotic individuals, conscientiousness is no remedy.

\subsubsection{Role of Pessimistic Expectations}

As an extension of the economic framework presented above, pessimistic expectations are now added to the model. Now, expected benefits $(B)$ are not only influenced by the individual personality, but also by pessimistic expectations $(\pi, 0<\pi<1)$ related to environmental factors (for instance, negative experiences in the family or school) that pose a production loss, independent of personality. Again, the individual aims to maximize own utility and can choose an effort level to achieve this. The utility function can be described such that:

$$
U(f, \pi, n, c)=c F(f)+(1-\pi)(1-c)(1-n) F(f)-\beta f
$$

Assuming that $F(f)=f^{\alpha}$ with $0<\alpha<1$ yields:

$$
U(f, \pi, n, c)=c f^{\alpha}+(1-\pi)(1-c)(1-n) f^{\alpha}-\beta f
$$


Figure 2.4: Optimal Effort and Pessimistic Expectations

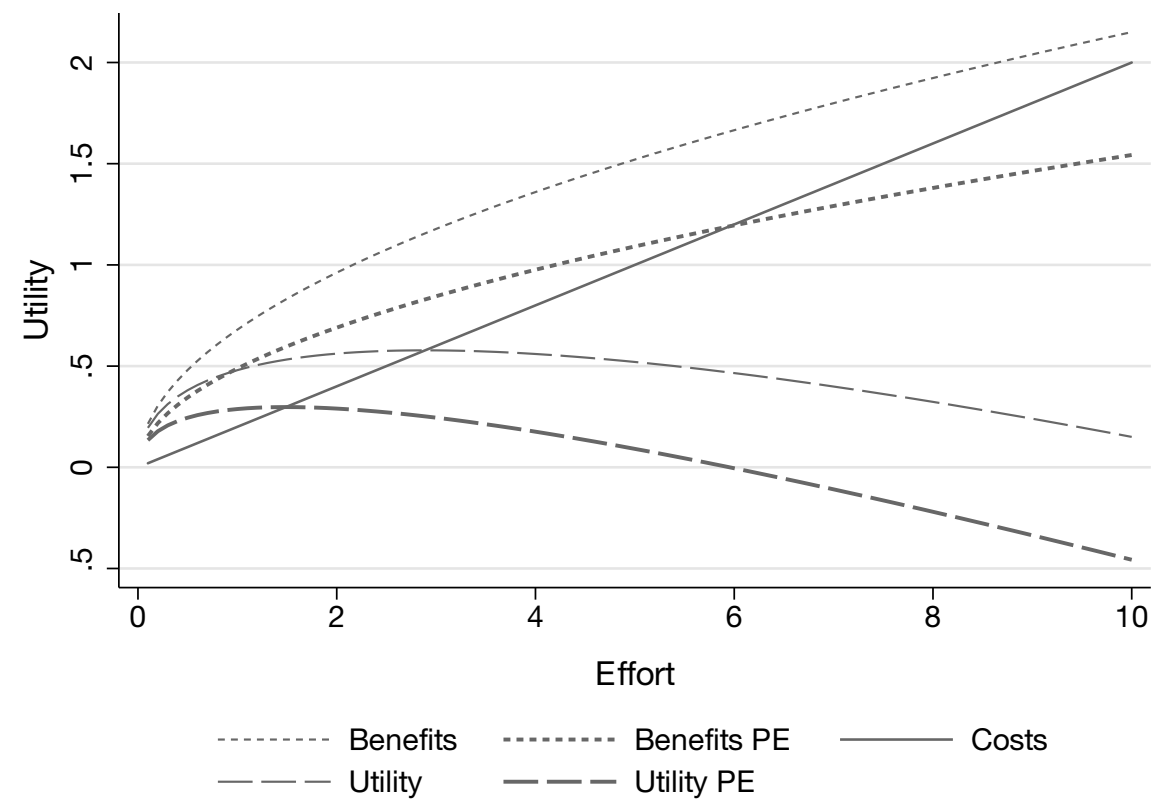

Note: Used parameters are $\alpha=0.5, \beta=0.2, c=0.2, n=0.4$ and $\pi=0.4$. Pessimistic Expectations $(\mathrm{PE})$.

Optimal effort $f^{*}$ can be derived:

$$
\begin{aligned}
\frac{\partial U}{\partial f} & =\alpha c f^{\alpha-1}+\alpha(1-\pi)(1-c)(1-n) f^{\alpha-1}-\beta=0 \\
f^{*} & =\left(\frac{\beta}{c \alpha+\alpha(1-\pi)(1-c)(1-n)}\right)^{\frac{1}{\alpha-1}}
\end{aligned}
$$

Figure 2.4 shows that the individual's optimal effort level is affected by personality, here neuroticism $(n)$ and conscientiousness $(c)$, and pessimistic expectations $(\pi)$ and that at any given level of personality traits, the expected benefits, the level of optimal effort and the optimal utility are lower for the pessimistic individual.

Taking the partial derivative of Equation 2.5 with respect to $n$ yields:

$$
\frac{\partial f^{*}}{\partial n}=\frac{1}{\alpha-1} \frac{\beta^{\frac{1}{\alpha-1}} \alpha(1-\pi)(1-c)}{(c \alpha+\alpha(1-\pi)(1-c)(1-n))^{\frac{\alpha}{\alpha-1}}}<0
$$


Figure 2.5: Optimal Effort and Pessimistic Expectations in Relation to Neuroticism and Conscientiousness
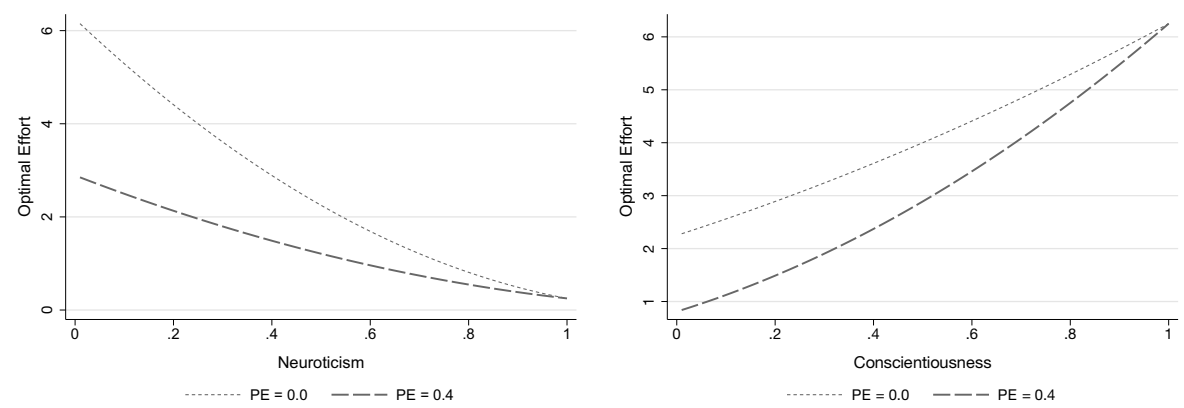

Note: Used parameters are $\alpha=0.5, \beta=0.2, c=0.2$ and $n=0.4$ respectively. Pessimistic Expectations $(\mathrm{PE})$.

Since $0<\alpha<1,0<\beta<1,0<c<1$ and $0<\pi<1$, it follows that the first term is negative and the second term is positive. Thus, $f_{n}^{*}<0$ and optimal effort decreases with neuroticism (Figure 2.5). Taking the partial derivative of Equation 2.5 with respect to $c$ yields:

$$
\frac{\partial f^{*}}{\partial c}=\frac{1}{\alpha-1} \frac{-\beta^{\frac{1}{\alpha-1}}(\alpha-\alpha(1-\pi)(1-n)}{(c \alpha+\alpha(1-\pi)(1-c)(1-n))^{\frac{\alpha}{\alpha-1}}}>0
$$

Since both the first and second term are negative, $f_{c}^{*}>0$ and optimal effort increases with conscientiousness (Figure 2.5). Figure 2.5 further depicts that the relation between personality and optimal effort changes if the individual has pessimistic expectations and that optimal effort is lower for the pessimistic individual. However, for highly neurotic individuals, there is now almost no additional decline in effort possible. Highly neurotic individuals are likely to have pessimistic beliefs, even without adverse environmental factors that might boost pessimistic expectations, whereby optimal effort is already very low for these individuals. On the other hand, this result suggests that highly neurotic individuals who receive environmental support to reduce and overcome pessimistic expectations are likely to have a higher optimal effort level.

Further, to derive the substitution effect of conscientiousness on optimal effort related to neuroticism, the second-order derivative of $f_{n}^{*}$ in response to a change in $c$ 
is calculated:

$$
\begin{aligned}
\frac{\partial^{2} f^{*}}{\partial n \partial c} & =-\alpha \beta^{\frac{1}{\alpha-1}}(1-\pi)\left(\frac{\alpha^{2}-c \alpha-\alpha(1-\pi)(1-c)(1-n)}{(\alpha-1)^{2}(c \alpha+\alpha(1-\pi)(1-c)(1-n))^{\frac{\alpha}{\alpha-1}+1}}\right) \\
& >0
\end{aligned}
$$

Given that both terms are negative, it is shown that the substitution effect of conscientiousness on optimal effort related to neuroticism $f_{n}^{*}$ is positive $\left(f_{n c}^{*}>0\right)$. This means that the effect of neuroticism differs depending on the level of conscientiousness and that at any level of neuroticism, optimal effort is higher with increasing conscientiousness (Figure 2.6).

Next, the relation between optimal utility $\left(U^{*}\right)$, pessimistic expectations $(\pi)$, neuroticism $(n)$ and conscientiousness $(c)$ is derived. Given that $A=(c+(1-\pi)(1-$ $c)(1-n))$, optimal utility can be written as:

$$
\begin{aligned}
U^{*}\left(f^{*}, \pi, n, c\right) & =c f^{* \alpha}+(1-\pi)(1-c)(1-n) f^{* \alpha}-\beta f^{*} \\
& =(c+(1-\pi)(1-c)(1-n)) f^{* \alpha}-\beta f^{*} \\
& =A f^{* \alpha}-\beta f^{*}
\end{aligned}
$$

Given that $A=(c+(1-\pi)(1-c)(1-n))$, optimal effort $f^{*}$ is:

$$
\begin{aligned}
f^{*} & =\beta^{\frac{1}{\alpha-1}}(c \alpha+\alpha(1-\pi)(1-c)(1-n))^{\frac{1}{1-\alpha}} \\
& =\beta^{\frac{1}{\alpha-1}}(\alpha A)^{\frac{1}{1-\alpha}}
\end{aligned}
$$

Substituting optimal effort $\left(f^{*}\right)$ into optimal utility $\left(U^{*}\right)$ yields:

$$
\begin{aligned}
U^{*}\left(f^{*}, \pi, n, c\right) & =A\left(\beta^{\frac{1}{\alpha-1}}(\alpha A)^{\frac{1}{1-\alpha}}\right)^{\alpha}-\beta \beta^{\frac{1}{\alpha-1}}(\alpha A)^{\frac{1}{1-\alpha}} \\
& =\beta^{\frac{1}{\alpha-1}} \alpha^{\frac{\alpha}{1-\alpha}}(1-\alpha) A^{\frac{1}{1-\alpha}}
\end{aligned}
$$

Given that $B=\beta^{\frac{1}{\alpha-1}} \alpha^{\frac{\alpha}{1-\alpha}}(1-\alpha)$, optimal utility can be written as:

$$
U^{*}\left(f^{*}, \pi, n, c\right)=B(A)^{\frac{1}{1-\alpha}}
$$


Figure 2.6: Effort Substitution Effect between Pessimistic Expectations, Neuroticism and Conscientiousness

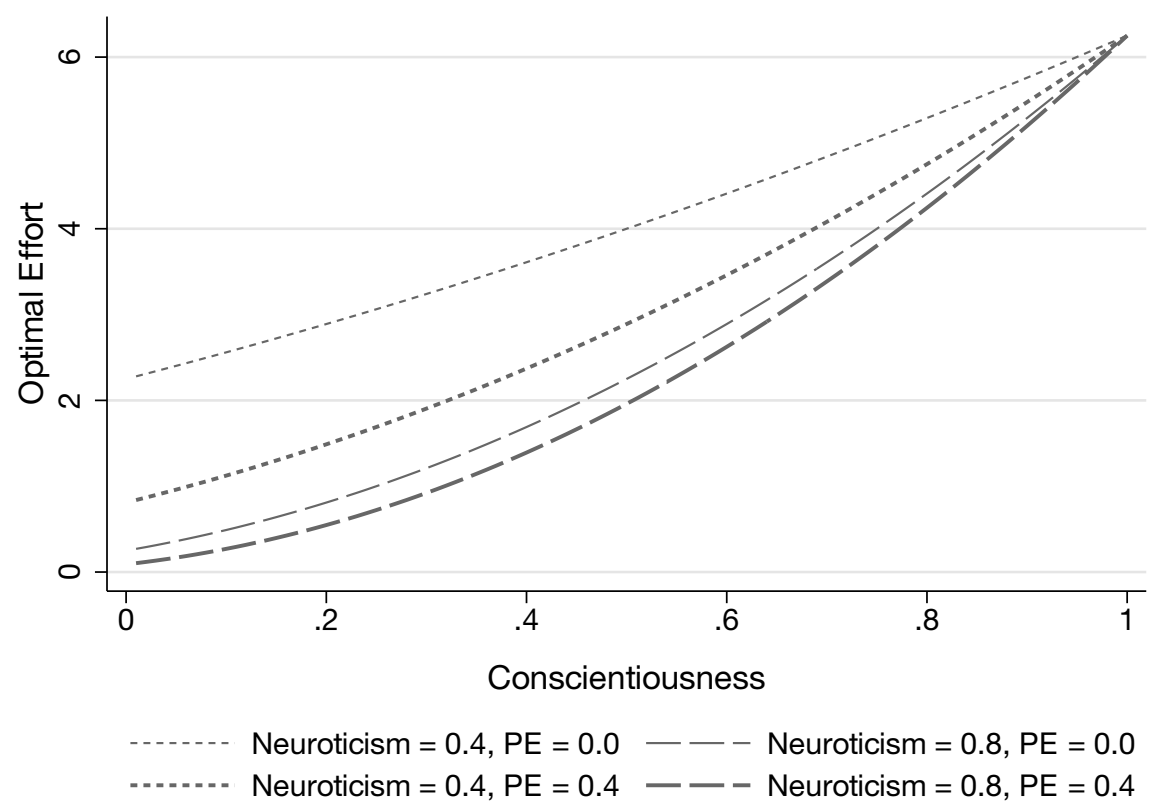

Note: Used parameters are $\alpha=0.5$ and $\beta=0.2$. Pessimistic Expectations (PE).

Taking the partial derivative of Equation 2.7 with respect to $n$ yields:

$$
\frac{\partial U^{*}}{\partial n}=-\beta^{\frac{1}{\alpha-1}}(1-\pi)(1-c)(\alpha c+\alpha(1-\pi)(1-c)(1-n))^{\frac{\alpha}{1-\alpha}}<0
$$

Given that $-\beta^{\frac{\alpha}{\alpha-1}}<0,(1-\pi)(1-c)>0$ and $(\alpha c+\alpha(1-\pi)(1-c)(1-n))^{\frac{\alpha}{1-\alpha}}>0$, it follows that $U_{n}^{*}<0$. Optimal utility decreases with neuroticism (see also discussion in Subsection 2.3.1). Taking the partial derivative of Equation 2.7 with respect to $c$ yields:

$$
\frac{d U^{*}}{d c}=\beta^{\frac{1}{\alpha-1}}(n(1-\pi)+\pi)(\alpha A)^{\frac{\alpha}{1-\alpha}}>0
$$

Given that $\left.\beta^{\frac{\alpha}{\alpha-1}}>0, n(1-\pi)+\pi\right)>0$ and $(\alpha A)^{\frac{\alpha}{1-\alpha}}$, it follows that $U_{c}^{*}>0$. Optimal utility increases with conscientiousness (see also discussion in Subsection 2.3.1). However, pessimistic expectations are likely to reduce the optimal effort level, 
independent of personality. Pessimistic expectations are likely to become a selffulfilling prophecy particularly for more neurotic and less conscientious individuals. Moreover, conscientiousness can potentially mitigate the negative relation between neuroticism and optimal utility. To observe this, $U_{n c}^{*}$ is derived. Given that $A=$ $(c+(1-\pi)(1-c)(1-n))$ and $C=-\beta^{\frac{1}{\alpha-1}} \alpha^{\frac{\alpha}{1-\alpha}}(1-\pi)$, this yields:

$$
\frac{\partial^{2} U^{*}}{\partial n \partial c}=C\left(\left(-1+\frac{\alpha(1-c)(n(1-\pi)+\pi)}{(1-\alpha)(c+(1-\pi)(1-c)(1-n))}\right) A^{\frac{\alpha}{1-\alpha}}\right)>0
$$

Given that $C<0$ and $A^{\frac{\alpha}{1-\alpha}}>0, U_{n c}^{*}>0$ if $\frac{\alpha(1-c)(n(1-\pi)+\pi)}{(1-\alpha)(c+(1-\pi)(1-c)(1-n))}<1$. Again, for high levels of $n$ or $\pi$ and low levels of $c$, the substitution effect is negative. This result suggests that for the highly neurotic individual, or alternatively, given severely adverse conditions, more conscientiousness is likely to be no remedy, but might even cause more harm.

\subsection{Conclusion}

The economic model presented here adds to human capital theory by considering variation in individual investment and utility related to personality differences and expectations. The theoretical considerations suggest that particularly low emotionally stable and low conscientious individuals who have pessimistic expectations are more likely to react with procrastination or avoidance in challenging situations (i.e. lower effort) based on short-run gains related to a reduction of negative emotions. However, these short-run benefits are likely to be outweighed by the long-run costs (i.e. lower utility).

Nevertheless, these short-term benefits as well as the self-fulfilling prophecy related to destructive expectations and negative experiences makes it likely that particularly this group at-risk unconsciously and repetitively uses maladaptive and ineffective coping strategies related to poor long-term outcomes. In other words, this group at-risk is likely to perceive procrastination or avoidance as beneficial and rational, whereby long-run costs and disadvantageous consequences might not be taken into consideration. Therefore, particularly low emotionally stable and low conscientious individuals who have pessimistic expectations are likely to be trapped in a selfsustained circle of multidimensional disadvantage. 
The economic framework provides an explanation for the empirical observation that low emotionally stable and low conscientious individuals are more likely to experience a socioeconomic disadvantage in life. It suggests that negatively biased expectations are likely to be related to reduced effort in challenging situations and an individual and social welfare loss. Furthermore, it indicates that low emotional stability is not inevitably related to a socioeconomic disadvantage and that both conscientiousness as well as more optimistic expectations might be a source of resilience.

Behavioral economists might interpret this scenario as variation in economic preferences. Accordingly, low emotionally stable individuals might be present-biased and prefer the short-run relief, while they discount possible future losses (Laibson, 1997). In terms of policy implications, this interpretation implies that no policy interventions are required, because the individual acts rationally according to own preferences.

In contrast, the economic framework supposes that the scenario should be rather interpreted as a rational, but biased choice than as naive hyperbolic discounting. ${ }^{12}$ In this context, the individual choice is considered as primarily rational, which is in line with the standard economic framework. However, it is biased, because the foresight of low emotionally stable individuals is likely to be more pessimistic compared to more emotionally stable individuals due to higher arousal and destructive thoughts. This scenario is related to market failure and suggests that a reduction of the expectation bias might improve socioeconomic outcomes (e.g., De Paola and Scoppa, 2015). Higher conscientiousness can be regarded as a proxy for a more organized and conscious mindset that is likely to reduce the noise adhered to process of decision-making and problem-solving.

$1 \overline{12 \text { This idea is related to a forthcoming }}$ paper by Gabaix and Laibson (2016). 


\subsection{Appendix}

In the following, all the equations are presented including more detailed calculation steps.

\subsubsection{Personality, Effort and Utility}

Utility function:

$$
U(f, n, c)=c f^{\alpha}+(1-c)(1-n) f^{\alpha}-\beta f
$$

Optimal effort:

$$
\begin{aligned}
\frac{\partial U}{\partial f} & =\alpha c f^{\alpha-1}+\alpha(1-c)(1-n) f^{\alpha-1}-\beta=0 \\
f^{\alpha-1} & =\frac{\beta}{c \alpha+\alpha(1-c)(1-n)} \\
f^{*} & =\left(\frac{\beta}{c \alpha+\alpha(1-c)(1-n)}\right)^{\frac{1}{\alpha-1}} \\
f^{*} & =\left(\frac{\beta}{\alpha(1-n+c n)}\right)^{\frac{1}{\alpha-1}}
\end{aligned}
$$


First-order partial derivative of optimal effort $\left(f^{*}\right)$ regarding neuroticism $(n)$ :

$$
\begin{aligned}
\frac{\partial f^{*}}{\partial n} & =\frac{1}{\alpha-1}\left(\frac{\beta}{\alpha(1-n+c n)}\right)^{\frac{1}{\alpha-1}-1} \frac{\alpha \beta(1-c)}{(\alpha(1-n+c n))^{2}} \\
& =\frac{1}{\alpha-1} \frac{\beta^{\frac{1}{\alpha-1}-1} \beta \alpha(1-c)}{(\alpha(1-n+c n))^{\frac{1}{\alpha-1}-1+2}} \\
& =\frac{1}{\alpha-1} \frac{\beta^{\frac{1}{\alpha-1}} \alpha(1-c)}{(\alpha(1-n+c n))^{\frac{\alpha}{\alpha-1}}} \\
& <0
\end{aligned}
$$

First-order partial derivative of optimal effort $\left(f^{*}\right)$ regarding conscientiousness $(c)$ :

$$
\begin{aligned}
\frac{\partial f^{*}}{\partial c} & =\frac{1}{\alpha-1}\left(\frac{\beta}{\alpha(1-n+c n)}\right)^{\frac{1}{\alpha-1}-1} \frac{-\alpha \beta n}{(\alpha(1-n+c n))^{2}} \\
& =\frac{1}{\alpha-1} \frac{-\beta^{\frac{1}{\alpha-1}} \alpha n}{(\alpha(1-n+c n))^{\frac{\alpha}{\alpha-1}}} \\
& >0
\end{aligned}
$$


Second-order partial derivative of optimal effort $\left(f^{*}\right)$ regarding neuroticism $(n)$ and conscientiousness $(c)$ :

$$
\begin{aligned}
& \frac{\partial^{2} f^{*}}{\partial n \partial c}=\frac{1}{\alpha-1} \\
& \frac{-\alpha \beta^{\frac{1}{\alpha-1}}(\alpha(1-n+c n))^{\frac{\alpha}{\alpha-1}}-\left(\alpha \beta^{\frac{1}{\alpha-1}}(1-c)\right)\left(\frac{\alpha}{\alpha-1}\right)}{(\alpha(1-n+c n))^{\frac{2 \alpha}{\alpha-1}}} \\
& \underline{\left.(\alpha(1-n+c n))^{\frac{\alpha}{\alpha-1}-1}(\alpha n)\right)} \\
& =\frac{1}{\alpha-1} \frac{\left.-\alpha \beta^{\frac{1}{\alpha-1}}-\left(\alpha \beta^{\frac{1}{\alpha-1}}(1-c)\right)\left(\frac{\alpha}{\alpha-1}\right)(\alpha(1-n+c n))^{-1}(\alpha n)\right)}{(\alpha(1-n+c n))^{\frac{\alpha}{\alpha-1}}} \\
& =\frac{1}{\alpha-1} \frac{\left.-\alpha \beta^{\frac{1}{\alpha-1}}(\alpha(1-n+c n))-\left(\alpha \beta^{\frac{1}{\alpha-1}}(1-c)\right)\left(\frac{\alpha}{\alpha-1}\right)(\alpha n)\right)}{(\alpha(1-n+c n))^{\frac{\alpha}{\alpha-1}+1}} \\
& =\frac{\left.-\alpha \beta^{\frac{1}{\alpha-1}}(\alpha(1-n+c n))-\left(\alpha \beta^{\frac{1}{\alpha-1}}(1-c)\right)\left(\frac{\alpha}{\alpha-1}\right)(\alpha n)\right)}{(\alpha-1)(\alpha(1-n+c n))^{\frac{\alpha}{\alpha-1}+1}} \\
& =-\alpha \beta^{\frac{1}{\alpha-1}} \frac{\alpha(1-n+c n)+(1-c) \frac{\alpha^{2} n}{\alpha-1}}{(\alpha-1)(\alpha(1-n+c n))^{\frac{\alpha}{\alpha-1}+1}} \\
& =-\alpha \beta^{\frac{1}{\alpha-1}} \frac{(\alpha-1)(\alpha(1-n+c n))+(1-c) \alpha^{2} n}{(\alpha-1)^{2}(\alpha(1-n+c n))^{\frac{\alpha}{\alpha-1}+1}} \\
& =-\alpha \beta^{\frac{1}{\alpha-1}} \frac{\alpha^{2}(1-n+c n)-\alpha(1-n+c n)+\alpha^{2} n-\alpha^{2} c n}{(\alpha-1)^{2}(\alpha(1-n+c n))^{\frac{\alpha}{\alpha-1}+1}} \\
& =-\alpha \beta^{\frac{1}{\alpha-1}}\left(\frac{\alpha^{2}-\alpha(1-n+c n)}{(\alpha-1)^{2}(\alpha(1-n+c n))^{\frac{\alpha}{\alpha-1}+1}}\right) \\
& >0
\end{aligned}
$$




\section{THEORETICAL CONSIDERATIONS}

First-order partial derivative of optimal utility $\left(U^{*}\right)$ regarding neuroticism $(n)$ :

$$
\begin{aligned}
\frac{\partial U^{*}}{\partial n} & =\beta^{\frac{\alpha}{\alpha-1}}(-1+c)(\alpha(1-n+c n))^{\frac{\alpha}{1-\alpha}} \\
& +\beta^{\frac{1}{\alpha-1}}(1-n+c n)\left(\frac{\alpha}{1-\alpha}\right)(\alpha(1-n+c n))^{\frac{\alpha}{1-\alpha}-1}(\alpha(-1+c)) \\
& -\beta^{\frac{1}{\alpha-1}}\left(\frac{1}{1-\alpha}\right)(\alpha(1-n+c n))^{\frac{1}{1-\alpha}-1}(\alpha(-1+c)) \\
& =\beta^{\frac{\alpha}{\alpha-1}}\left(-(1-c)(\alpha(1-n+c n))^{\frac{\alpha}{1-\alpha}}\right. \\
& \left.+\frac{(\alpha(1-n+c n))(-\alpha(1-c))}{1-\alpha}\right)(\alpha(1-n+c n))^{\frac{\alpha}{1-\alpha}-1} \\
& \left.-\frac{-\alpha(1-c)}{1-\alpha}(\alpha(1-n+c n))^{\frac{1}{1-\alpha}-1}\right) \\
& =\beta^{\frac{\alpha}{\alpha-1}}\left(-(1-c)(\alpha(1-n+c n))^{\frac{\alpha}{1-\alpha}}\right. \\
& +\frac{(\alpha(1-n+c n))(-\alpha(1-c)) \frac{(\alpha(1-n+c n))^{\frac{\alpha}{1-\alpha}}}{\alpha(1-n+c n)}}{1-\alpha} \\
& \left.-\frac{\alpha(1-c)}{1-\alpha}(\alpha(1-n+c n))^{\frac{\alpha}{1-\alpha}}+\frac{\alpha(1-c)}{1-\alpha}(\alpha(1-n+c n))^{\frac{\alpha}{1-\alpha}}\right) \\
& -\alpha(1-c) \\
& \left.\left.=\frac{\alpha}{1-\alpha}(1-n+c n)\right)^{\frac{\alpha}{1-\alpha}}\right)
\end{aligned}
$$


First-order partial derivative of optimal utility $\left(U^{*}\right)$ regarding conscientiousness $(c)$ :

$$
\begin{aligned}
\frac{\partial U^{*}}{\partial c} & =\beta^{\frac{\alpha}{\alpha-1}}\left(n(\alpha(1-n+c n))^{\frac{\alpha}{1-\alpha}}\right. \\
& +(1-n+c n)\left(\frac{\alpha}{1-\alpha}\right)(\alpha(1-n+c n))^{\frac{\alpha}{1-\alpha}-1} \alpha n \\
& \left.-\left(\frac{1}{1-\alpha}\right)(\alpha(1-n+c n))^{\frac{1}{1-\alpha}-1} \alpha n\right) \\
& =\beta^{\frac{\alpha}{\alpha-1}}\left(n(\alpha(1-n+c n))^{\frac{\alpha}{1-\alpha}}\right. \\
& +\left(\frac{\alpha n}{1-\alpha}\right)(\alpha(1-n+c n))^{\frac{\alpha}{1-\alpha}} \\
& \left.-\left(\frac{\alpha n}{1-\alpha}\right)(\alpha(1-n+c n))^{\frac{\alpha}{1-\alpha}}\right) \\
& =n \beta^{\frac{\alpha}{\alpha-1}}\left(\alpha(1-n+c n)^{\frac{\alpha}{1-\alpha}}\right) \\
& >0
\end{aligned}
$$




\section{THEORETICAL CONSIDERATIONS}

Second-order partial derivative of optimal utility $\left(U^{*}\right)$ regarding neuroticism $(n)$ and conscientiousness $(c)$ :

$$
\begin{aligned}
& \frac{\partial^{2} U^{*}}{\partial n \partial c}=\beta^{\frac{\alpha}{\alpha-1}}(\alpha(1-n+c n))^{\frac{\alpha}{1-\alpha}}-\beta^{\frac{\alpha}{1-\alpha}}(1-c)\left(\frac{\alpha}{1-\alpha}\right) \\
& (\alpha(1-n+c n))^{\frac{\alpha}{1-\alpha}-1}(\alpha n) \\
& =\beta^{\frac{\alpha}{\alpha-1}} \\
& \left((\alpha(1-n+c n))^{\frac{\alpha}{1-\alpha}}-\left(\frac{n \alpha^{2}(1-c)}{1-\alpha}\right)(\alpha(1-n+c n))^{\frac{\alpha}{1-\alpha}-1}\right) \\
& =\beta^{\frac{\alpha}{\alpha-1}}\left((\alpha(1-n+c n))^{\frac{\alpha}{1-\alpha}}-\frac{n \alpha^{2}(1-c)(\alpha(1-n+c n))^{\frac{\alpha}{1-\alpha}}}{(1-\alpha)(\alpha(1-n+c n)}\right) \\
& =\beta^{\frac{\alpha}{\alpha-1}}\left((\alpha(1-n+c n))^{\frac{\alpha}{1-\alpha}}-\frac{n \alpha(1-c)(\alpha(1-n+c n))^{\frac{\alpha}{1-\alpha}}}{(1-\alpha)(1-n+c n)}\right) \\
& =\beta^{\frac{\alpha}{\alpha-1}} \\
& \left((\alpha(1-n+c n))^{\frac{\alpha}{1-\alpha}}-\frac{n \alpha(1-c)}{(1-\alpha)(1-n+c n)}(\alpha(1-n+c n))^{\frac{\alpha}{1-\alpha}}\right) \\
& =\beta^{\frac{\alpha}{\alpha-1}}\left(1-\frac{n \alpha(1-c)}{(1-\alpha)(1-n+c n)}(\alpha(1-n+c n))^{\frac{\alpha}{1-\alpha}}\right) \\
& =\beta^{\frac{\alpha}{\alpha-1}}\left(\frac{1-\alpha-n+c n}{1-\alpha-n+c n+\alpha n-\alpha c n}(\alpha(1-n+c n))^{\frac{\alpha}{1-\alpha}}\right) \\
& >0
\end{aligned}
$$




\subsubsection{Role of Pessimistic Expectations}

Utility function:

$$
U(f, \pi, n, c)=c f^{\alpha}+(1-\pi)(1-c)(1-n) f^{\alpha}-\beta f
$$

Optimal effort:

$$
\begin{aligned}
\frac{\partial U}{\partial f} & =\alpha c f^{\alpha-1}+\alpha(1-\pi)(1-c)(1-n) f^{\alpha-1}-\beta=0 \\
f^{\alpha-1} & =\frac{\beta}{c \alpha+\alpha(1-\pi)(1-c)(1-n)} \\
f^{*} & =\left(\frac{\beta}{c \alpha+\alpha(1-\pi)(1-c)(1-n)}\right)^{\frac{1}{\alpha-1}}
\end{aligned}
$$

First-order partial derivative of optimal effort $\left(f^{*}\right)$ regarding neuroticism $(n)$ :

$$
\begin{aligned}
\frac{\partial f^{*}}{\partial n}= & \frac{1}{\alpha-1}\left(\frac{\beta}{c \alpha+\alpha(1-\pi)(1-c)(1-n)}\right)^{\frac{1}{\alpha-1}-1} \\
& \frac{\alpha \beta(1-\pi)(1-c)}{(c \alpha+\alpha(1-\pi)(1-c)(1-n))^{2}} \\
= & \frac{1}{\alpha-1} \frac{\beta^{\frac{1}{\alpha-1}-1} \beta \alpha(1-\pi)(1-c)}{(c \alpha+\alpha(1-\pi)(1-c)(1-n))^{\frac{1}{\alpha-1}-1+2}} \\
= & \frac{1}{\alpha-1} \frac{\beta^{\frac{1}{\alpha-1}} \alpha(1-\pi)(1-c)}{(c \alpha+\alpha(1-\pi)(1-c)(1-n))^{\frac{\alpha}{\alpha-1}}} \\
< & 0
\end{aligned}
$$


First-order partial derivative of optimal effort $\left(f^{*}\right)$ regarding conscientiousness $(c)$ :

$$
\begin{aligned}
\frac{\partial f^{*}}{\partial c}= & \frac{1}{\alpha-1}\left(\frac{\beta}{c \alpha+\alpha(1-\pi)(1-c)(1-n)}\right)^{\frac{1}{\alpha-1}-1} \\
& \frac{-\beta(\alpha-\alpha(1-\pi)(1-n))}{(c \alpha+\alpha(1-\pi)(1-c)(1-n))^{2}} \\
= & \frac{1}{\alpha-1} \frac{-\beta^{\frac{1}{\alpha-1}}(\alpha-\alpha(1-\pi)(1-n))}{(c \alpha+\alpha(1-\pi)(1-c)(1-n))^{\frac{\alpha}{\alpha-1}}} \\
> & 0
\end{aligned}
$$

The second-order partial derivative of optimal effort $\left(f^{*}\right)$ regarding neuroticism $(n)$ and conscientiousness $(c)$ is derived as follows. The first-order partial derivative of optimal effort $\left(f^{*}\right)$ regarding neuroticism $(n)$ is:

$$
\frac{\partial f^{*}}{\partial n}=\frac{1}{\alpha-1} \frac{\beta^{\frac{1}{\alpha-1}} \alpha(1-\pi)(1-c)}{(c \alpha+\alpha(1-\pi)(1-c)(1-n))^{\frac{\alpha}{\alpha-1}}}
$$

Given that $A=\alpha c+\alpha(1-\pi)(1-c)(1-n), f_{n c}^{*}$ is:

$$
\begin{aligned}
\frac{\partial^{2} f^{*}}{\partial n \partial c}= & \frac{1}{\alpha-1} \frac{-\alpha \beta^{\frac{1}{\alpha-1}}(1-\pi)(A)^{\frac{\alpha}{\alpha-1}}-\left(\frac{\frac{\alpha}{\alpha-1}(A)^{\frac{\alpha}{\alpha-1}}(\alpha-\alpha(1-\pi)(1-n))}{A}\right)}{(A)^{2 \frac{\alpha}{\alpha-1}}} \\
& \frac{\left(\beta^{\frac{1}{\alpha-1}} \alpha(1-\pi)(1-c)\right)}{A}
\end{aligned}
$$

Continued on next page ... 


$$
\begin{aligned}
& =\frac{1}{\alpha-1} \\
& \frac{-\alpha \beta^{\frac{1}{\alpha-1}}(1-\pi)-\left(\frac{\frac{\alpha}{\alpha-1}(\alpha-\alpha(1-\pi)(1-n))}{A}\right)\left(\beta^{\frac{1}{\alpha-1}} \alpha(1-\pi)(1-c)\right)}{(A)^{\frac{\alpha}{\alpha-1}}} \\
& =\frac{1}{\alpha-1} \frac{-\alpha \beta^{\frac{1}{\alpha-1}}(1-\pi)\left(A+\frac{\alpha}{\alpha-1}(\alpha-\alpha(1-\pi)(1-n))(1-c)\right.}{(A)^{\frac{\alpha}{\alpha-1}}+1} \\
& =\frac{-\alpha \beta^{\frac{1}{\alpha-1}}(1-\pi)\left(A+\frac{\alpha}{\alpha-1}(\alpha-\alpha(1-\pi)(1-n))(1-c)\right.}{(\alpha-1)(A)^{\frac{\alpha}{\alpha-1}+1}} \\
& =-\alpha \beta^{\frac{1}{\alpha-1}}(1-\pi) \frac{(\alpha-1) A+\alpha(\alpha-\alpha(1-\pi)(1-n))(1-c)}{(\alpha-1)^{2}(A)^{\frac{\alpha}{\alpha-1}+1}} \\
& =-\alpha \beta^{\frac{1}{\alpha-1}}(1-\pi) \frac{(\alpha-1) A+\alpha^{2}(1-c)-\alpha^{2}(1-\pi)(1-c)(1-n)}{(\alpha-1)^{2}(A)^{\frac{\alpha}{\alpha-1}+1}} \\
& =-\alpha \beta^{\frac{1}{\alpha-1}}(1-\pi) \frac{(\alpha-1) A+\alpha^{2}-\alpha(\alpha c+\alpha(1-\pi)(1-c)(1-n))}{(\alpha-1)^{2}(A)^{\frac{\alpha}{\alpha-1}+1}} \\
& =-\alpha \beta^{\frac{1}{\alpha-1}}(1-\pi) \frac{(\alpha-1) A+\alpha^{2}-\alpha A}{(\alpha-1)^{2}(A)^{\frac{\alpha}{\alpha-1}+1}} \\
& =-\alpha \beta^{\frac{1}{\alpha-1}}(1-\pi) \frac{\alpha A-A+\alpha^{2}-\alpha A}{(\alpha-1)^{2}(A)^{\frac{\alpha}{\alpha-1}+1}} \\
& =-\alpha \beta^{\frac{1}{\alpha-1}}(1-\pi) \frac{\alpha^{2}-A}{(\alpha-1)^{2}(A)^{\frac{\alpha}{\alpha-1}+1}} \\
& =-\alpha \beta^{\frac{1}{\alpha-1}}(1-\pi) \frac{\alpha^{2}-(\alpha c+\alpha(1-\pi)(1-c)(1-n))}{(\alpha-1)^{2}(\alpha c+\alpha(1-\pi)(1-c)(1-n))^{\frac{\alpha}{\alpha-1}+1}} \\
& >0
\end{aligned}
$$




\section{THEORETICAL CONSIDERATIONS}

The first-order partial derivative of optimal utility $\left(U^{*}\right)$ regarding neuroticism $(n)$ is derived as follows. Given that $A=(c+(1-\pi)(1-c)(1-n))$, the utility function is:

$$
\begin{aligned}
U(f, \pi, n, c) & =c f^{\alpha}+(1-\pi)(1-c)(1-n) f^{\alpha}-\beta f \\
& =(c+(1-\pi)(1-c)(1-n)) f^{\alpha}-\beta f \\
& =A f^{\alpha}-\beta f
\end{aligned}
$$

Given that $A=(c+(1-\pi)(1-c)(1-n))$, the optimal effort is:

$$
\begin{aligned}
f^{*} & =\left(\frac{\beta}{c \alpha+\alpha(1-\pi)(1-c)(1-n)}\right)^{\frac{1}{\alpha-1}} \\
& =\beta^{\frac{1}{\alpha-1}}\left(c \alpha+\alpha(1-\pi)(1-c)(1-n)^{\frac{-1}{\alpha-1}}\right. \\
& =\beta^{\frac{1}{\alpha-1}}\left(c \alpha+\alpha(1-\pi)(1-c)(1-n)^{\frac{1}{1-\alpha}}\right. \\
& =\beta^{\frac{1}{\alpha-1}}(\alpha A)^{\frac{1}{1-\alpha}}
\end{aligned}
$$

Including the optimal effort $f^{*}$ into the utility function and given that $B=\beta^{\frac{1}{\alpha-1}} \alpha^{\frac{\alpha}{1-\alpha}}(1-$ $\alpha)$ yields:

$$
\begin{aligned}
U(f, \pi, n, c) & =A\left(\beta^{\frac{1}{\alpha-1}}(\alpha A)^{\frac{1}{1-\alpha}}\right)^{\alpha}-\beta \beta^{\frac{1}{\alpha-1}}(\alpha A)^{\frac{1}{1-\alpha}} \\
& =A\left(\beta^{\frac{1}{\alpha-1}}(\alpha A)^{\frac{\alpha}{1-\alpha}}\right)-\beta^{\frac{\alpha}{\alpha-1}}(\alpha A)^{\frac{1}{1-\alpha}} \\
& =\beta^{\frac{1}{\alpha-1}}\left(A(\alpha A)^{\frac{\alpha}{1-\alpha}}-(\alpha A)^{\frac{1}{1-\alpha}}\right) \\
& =\beta^{\frac{1}{\alpha-1}}\left(\alpha^{\frac{\alpha}{1-\alpha}} A A^{\frac{\alpha}{1-\alpha}}-\alpha^{\frac{1}{1-\alpha}} A^{\frac{1}{1-\alpha}}\right) \\
& =\beta^{\frac{1}{\alpha-1}}\left(\alpha^{\frac{\alpha}{1-\alpha}} A^{\frac{1}{1-\alpha}}-\alpha^{\frac{1}{1-\alpha}} A^{\frac{1}{1-\alpha}}\right) \\
& =\beta^{\frac{1}{\alpha-1}}\left(\alpha^{\frac{\alpha}{1-\alpha}}(1-\alpha)\right)\left(A^{\frac{1}{1-\alpha}}\right) \\
& =B\left(A^{\frac{1}{1-\alpha}}\right)
\end{aligned}
$$


Given that $A_{n}^{\prime}=-(1-\pi)(1-c)$, the optimal utility regarding neuroticism $(n)$ is:

$$
\begin{aligned}
\frac{\partial U^{*}}{\partial n} & =B \frac{1}{1-\alpha} A^{\frac{1}{1-\alpha}-1} A_{n}^{\prime} \\
& =B \frac{1}{1-\alpha} A^{\frac{1}{1-\alpha}-1}(-(1-\pi)(1-c)) \\
& =B \frac{1}{1-\alpha} A^{\frac{\alpha}{1-\alpha}}(-(1-\pi)(1-c)) \\
& =\beta^{\frac{1}{\alpha-1}} \alpha^{\frac{\alpha}{1-\alpha}} A^{\frac{\alpha}{1-\alpha}}(-(1-\pi)(1-c)) \\
& =-\beta^{\frac{1}{\alpha-1}}(1-\pi)(1-c) \alpha A^{\frac{\alpha}{1-\alpha}} \\
& =-\beta^{\frac{1}{\alpha-1}}(1-\pi)(1-c)(\alpha c+\alpha(1-\pi)(1-c)(1-n))^{\frac{\alpha}{1-\alpha}} \\
& <0
\end{aligned}
$$

Given that $U^{*}=B\left(A^{\frac{1}{1-\alpha}}\right)$ and $A_{c}^{\prime}=1-(1-\pi)(1-n)=n+\pi-\pi n=n(1-\pi)+\pi$, the first-order partial derivative of optimal utility $\left(U^{*}\right)$ regarding conscientiousness $(c)$ :

$$
\begin{aligned}
\frac{\partial U^{*}}{\partial c} & =B \frac{1}{1-\alpha} A^{\frac{1}{1-\alpha}-1} A_{c}^{\prime} \\
& =B \frac{1}{1-\alpha} A^{\frac{1}{1-\alpha}-1}(n(1-\pi)+\pi) \\
& =\beta^{\frac{1}{\alpha-1}} \alpha^{\frac{\alpha}{1-\alpha}} A^{\frac{\alpha}{1-\alpha}}(n(1-\pi)+\pi) \\
& =\beta^{\frac{1}{\alpha-1}}(n(1-\pi)+\pi)(\alpha A)^{\frac{\alpha}{1-\alpha}} \\
& >0
\end{aligned}
$$




\section{THEORETICAL CONSIDERATIONS}

Finally, the second-order partial derivative of optimal utility $\left(U^{*}\right)$ regarding neuroti$\operatorname{cism}(n)$ and conscientiousness $(c)$ is derived. Given that $C=-\beta^{\frac{1}{\alpha-1}} \alpha^{\frac{\alpha}{1-\alpha}}(1-\pi)$, the the optimal utility regarding neuroticism $(n)$ is:

$$
\begin{gathered}
\frac{\partial U^{*}}{\partial n}=-\beta^{\frac{1}{\alpha-1}}(1-\pi)(1-c) \alpha A^{\frac{\alpha}{1-\alpha}} \\
=-\beta^{\frac{1}{\alpha-1}} \alpha^{\frac{\alpha}{1-\alpha}}(1-\pi)(1-c) A^{\frac{\alpha}{1-\alpha}} \\
=C(1-c) A^{\frac{\alpha}{1-\alpha}} \\
\frac{\partial^{2} U^{*}}{\partial n \partial c}=C\left(-A^{\frac{\alpha}{1-\alpha}}+(1-c)\left(\frac{\alpha}{1-\alpha}\right) A^{\frac{\alpha}{1-\alpha}-1} A_{c}^{\prime}\right) \\
=C\left(-A^{\frac{\alpha}{1-\alpha}}+\frac{\alpha(1-c) A_{c}^{\prime}}{(1-\alpha) A} A^{\frac{\alpha}{1-\alpha}}\right) \\
=C\left(-A^{\frac{\alpha}{1-\alpha}}+\frac{\alpha(1-c) A_{c}^{\prime}}{(1-\alpha) A} A^{\frac{\alpha}{1-\alpha}}\right) \\
=C\left(\left(-1+\frac{\alpha(1-c) A_{c}^{\prime}}{(1-\alpha) A} A^{\frac{\alpha}{1-\alpha}}\right)\right. \\
=C\left(\left(-1+\frac{\alpha(1-c)(n(1-\pi)+\pi)}{(1-\alpha)(c+(1-\pi)(1-c)(1-n))}\right) A^{\frac{\alpha}{1-\alpha}}\right)
\end{gathered}
$$

Given that $C=-\beta^{\frac{1}{\alpha-1}} \alpha^{\frac{\alpha}{1-\alpha}}(1-\pi)<0$ and $A^{\frac{\alpha}{1-\alpha}}=(c+(1-\pi)(1-c)(1-n))^{\frac{\alpha}{1-\alpha}}>0$, $\frac{\partial^{2} U^{*}}{\partial n \partial c}>0$ if $\frac{\alpha(1-c)(n(1-\pi)+\pi)}{(1-\alpha)(c+(1-\pi)(1-c)(1-n))}<1$. 


\section{CHAPTER 3}

\section{PERSONALITY AND}

\section{EDUCATIONAL ACHIEVEMENT*}

\footnotetext{
* The author received valuable comments from Arnaud Chevalier, Bart Golsteyn, Oliver Lüdtke and seminar participants at the "2016 LEAD Retreat" at the University Tübingen as well as at the "2017 PhD Conference in Behavioral Science" at the University College Dublin.
} 


\subsection{Introduction}

To what extent is there a relation between the personality traits neuroticism and conscientiousness and later life outcomes? This question is addressed by focusing on the objective measure of educational achievement at the age of 30. Education outcomes in adulthood can be considered as the aggregated result of many challenges individuals face in school or further education. If the theory presented in Chapter 2 holds, educational achievement at the age of 30 should vary with emotional stability and conscientiousness - also after controlling for cognitive ability and socioeconomic background - according to the hypothesized lines. To test this, the predictive power of (1) low emotional stability at the age of 16 for educational achievement at the age of 30 as well as the possible substitution effect of conscientiousness and (2) a change in emotional stability and conscientiousness between the ages of 10 and 16 and educational achievement at the age of 30 is analyzed.

Given that personality traits are considered mostly rank-order stable at adult age (Roberts and Del Vecchio, 2000; Borghans et al., 2008), it is hypothesized that the relation between emotional stability, conscientiousness and educational achievement remains relatively robust throughout life, because groups at-risk are likely to be trapped in less successful patterns of high arousal and avoidance behavior (e.g., Fouarge et al., 2013).

There is growing awareness in education economics that not only the individuals' intelligence is important for achievement in school and life, but that personality traits also play an important role (Heckman and Rubinstein, 2001). Borghans et al. (2011) argue that "grades and achievement test scores predict adult outcomes better than IQ because they also capture personality traits" (p.318; see also Borghans, Golsteyn, Heckman and Humphries, 2016). Economic studies show that low emotional stability reduces exam grades and education participation, while conscientiousness is related to improved results (Baron and Cobb-Clark, 2010; Lundberg, 2013; Ryan et al., 2013; Golsteyn and Schils, 2014; Mendolia and Walker, 2014b). However, the mechanisms behind these relations are far less explored. Gaining insights into these relations can help to identify high-risk individuals with regard to personality characteristics, which might yield important input for successful (pre-)intervention programs. In the next sections, the data are introduced and the results are discussed. The final section concludes. 


\subsection{Data}

The study is based on the BCS70, which provides detailed information about the individual's family background, cognitive ability, individual personality as well as educational achievement. Appendix Table 3.6 gives an overview of the overall number of observations and the variables investigated in each of the waves.

The analysis relies on a working sample restricted to cases with available information on cognitive ability at the age of 10 , personality at the ages of 10 and 16, mother's age, socioeconomic background and region of living at birth as well as educational achievement at the age of 30 . Only individuals who have no missing values are taken into account which results in 4203 observations (Appendix Table 3.7).

The issue of sample selection is addressed by comparing the working sample with the unrestricted sample (Appendix Table 3.7). The procedure yields that the working sample is positively selected. On average, people in the working sample less commonly show potentially problematic characteristics such as low emotional stability or low conscientiousness, but have higher cognitive ability, are from a more advantaged socioeconomic background, and have slightly older mothers. This is an intuitive result given that low conscientious people are less likely to continuously participate in the survey. The result further suggests that the individuals who are not covered at all by the survey presumably have even worse characteristics. Therefore, it is likely to estimate lower bound results and that the observed associations are even larger in the unrestricted sample.

To test the robustness of the results, multiple imputation of missing values with regard to the personality trait variables is performed. Personality item are imputed if there are five or fewer missing values out of 22 items. In this and the following chapters, the procedure is conducted by using multivariate imputation by chained equations. Imputation of missing values results in an increase of the sample size to 4908 observations. The results are consistent with the main results and available in Appendix Subsection 3.5.2.1. 
Figure 3.1: Educational Achievement (NVQ) at Age 30

Female
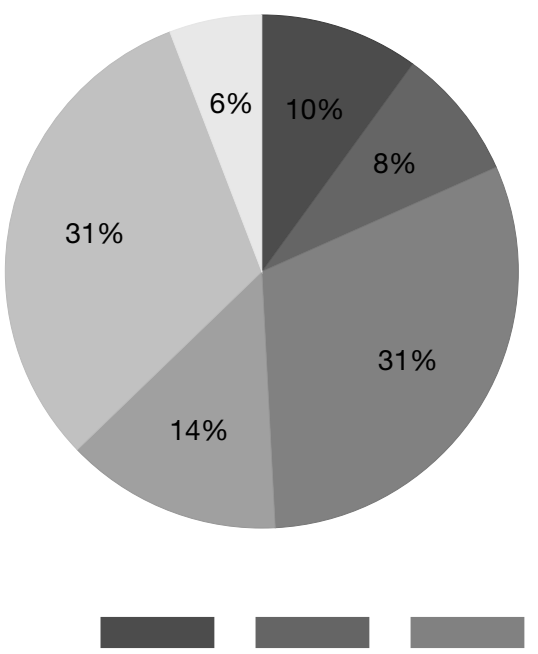

0

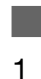

1

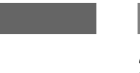

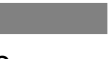

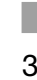

3
Male

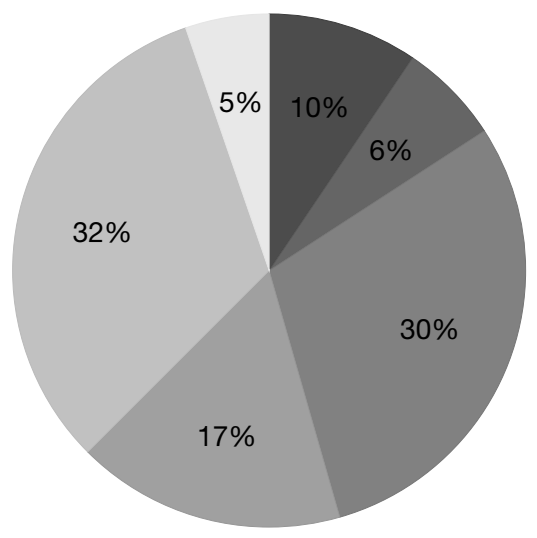

4

\section{5}

Source: BCS70. Own calculations, $\mathrm{n}=4203$.

\subsubsection{Educational Achievement}

Three dependent variables are used in the analysis. First, the highest National Vocational Qualification (NVQ) at the age of 30 is analyzed which comprises both academic and vocational qualifications. NVQ 0 means no qualification level reached, NVQ 1 equals a low Ordinary Level (O level) or a Certificate of Secondary Education (CSE) at grades 2 to 5, NVQ 2 includes a good O level, two or more AS (Advanced Subsidiary) levels or one A (Advanced) level, NVQ 3 equals more than one A level, NVQ 4 refers to a bachelor's or master's degree or PGCE (Postgraduate Certificate in Education) and NVQ 5 means higher degree such as doctorate (Dodgeon et al., 2011). Second, a binary variable that specifically accounts for the low educational achievers is created. The variable is 1 if the respondent has only reached NVQ 0 or 1 , while it is 0 if the respondent has reached NVQ 2 or higher at the age of 30. Finally, a binary measure of reaching an academic degree is constructed. The variable is 1 if the respondent has reached NVQ 4 or 5 , while it is 0 if the respondent has NVQ 
$0,1,2$ or 3 at the age of 30 .

Figure 3.1 provides information about the distribution of educational achievement at the age of 30 . It reveals that on average $17 \%$ of the working sample respondents only reach NVQ 0 or 1, 46\% have NVQ 2 or 3 and $37 \%$ reach NVQ 4 or 5 at the age of 30. To check the plausibility of the results, the working sample is compared with data from Eurostat (2017). In the Eurostat data in 2010, $22 \%$ of the 35 to 45 year olds reach less than primary or lower secondary education, while $37 \%$ of this age group have finished tertiary education. Differences with regard to low achievers might be explained by positive selection of respondents in the working sample, but also possible further education after the age of 30 .

\subsubsection{Personality Traits}

Throughout the dissertation, the focus lies on the personality traits neuroticism and conscientiousness. Neuroticism is defined as "the degree to which a person experiences the world as threatening. Lower-order facets are anxiety, angry hostility, depression, self-consciousness, impulsiveness and vulnerability" (Chapter 2 Table 2.1). The dimensions anxiety and impulsiveness are of particular interest, because they are linked to emotion-focused problem solving, feelings of arousal and pessimistic expectations. These dimensions are measured by the items "irritable", "miserable and tearful", "requests must be met immediately", "sullen or sulky", "changes mood quickly and drastically" or "outburst of temper unpredictable" (Appendix Table $3.10)$.

Conscientiousness is defined as "the degree to which a person is willing to comply with conventional rules. Low-order facets are competence, order, dutifulness, achievement, striving, self-discipline and deliberation" (Chapter 2 Table 2.1). The dimensions "self-discipline and deliberation" are of particular interest, because they are linked to problem-focused coping strategies, finishing tasks and reaching own goals. These dimensions are measured by the items "cannot settle", "inattentive, easily distracted", "fails to finish things" and "difficulty concentrating on task" (Appendix Table 3.10).

The BCS70 does not directly provide information about the personality traits emotional stability, conscientiousness, agreeableness and extraversion. In order to assess the personality of the individuals in the analysis, the study follows Prevoo and ter 
Weel (2015), who use BCS70 mother-rated behavioral items at the ages of 10 and 16 and extract emotional stability, conscientiousness, extraversion and agreeableness from an initial set of 38 behavioral items. To test the validity of the personality trait structure, a principle component factor analysis is calculated by using the proposed 22 standardized items. The first four factors have eigenvalues larger than one and most items load highly on their respective factors. ${ }^{13}$ The results are provided in Appendix Tables 3.8 and 3.9. Each trait is assessed by extracting the first principle component from the set of items belonging to that trait. The procedure is conducted with the behavioral items at the ages of 10 and 16 to derive personality variables at these ages. An overview of the reliability measures that correspond to the four personality traits is provided in Appendix Table 3.10. The results are comparable to those reported by Prevoo and ter Weel (2015).

In the analysis, personality traits at the ages of 10 and 16 are used threefold: First, they are used as continuous variables as shown in Figures 3.2 and 3.3. Second, four personality types capturing differences in emotional stability and conscientiousness are created. Thereby, the distribution of the standardized variables emotional stability and conscientiousness is split at the mean, i.e. at zero, and four above- and belowmean combinations of emotional stability and conscientiousness are constructed: (1) more emotionally stable and more conscientious, (2) more emotionally stable and less conscientious, (3) less emotionally stable and more conscientious, and (4) less emotionally stable and less conscientious individuals (Appendix Table 3.12). Third, the change in personality between the ages of 10 and 16 is used by subtracting the standardized personality score at the age of 10 from the standardized score at the age of 16 (Appendix Table 3.1).

Figures 3.2 and 3.3 present the distribution of personality traits at the ages of 10 and 16 by gender. The graphs show that on average individuals change their personality characteristics between the ages of 10 and 16, which is in line with the literature (Robins et al., 2001). Focusing on the personality traits emotional stability and conscientiousness, Appendix Table 3.10 shows a rank-order stability of around

\footnotetext{
13 The variables "often disobedient" and "not much liked by others" have also high loadings for another factor than the respective one which is in line with Prevoo and ter Weel (2015). However, due to theoretical considerations, they are assigned to the respective factors. For the item "interferes with others", the principle component analysis shows that the item refers to the factor agreeableness at the age of 10 , while it refers to emotional stability at the of 16 . Since the differences in loading are rather low, the item is sorted into the factor agreeableness at both ages.
} 
Figure 3.2: Personality Traits at Age 10
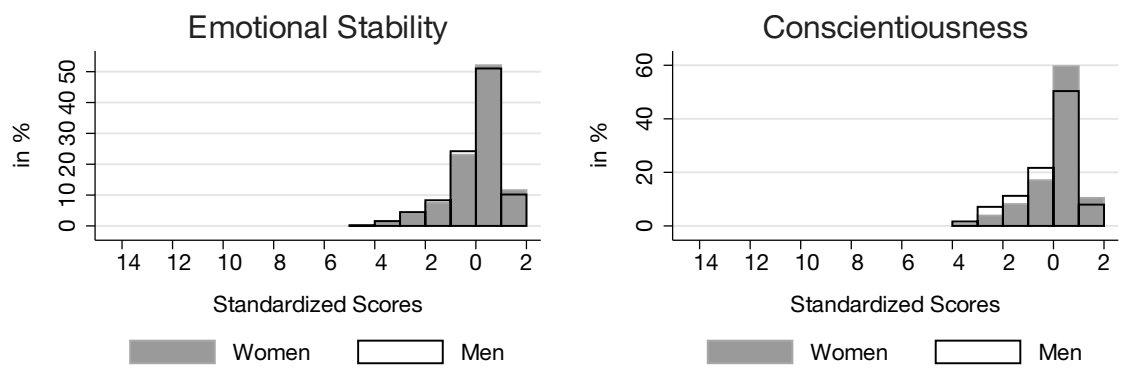

Agreeableness
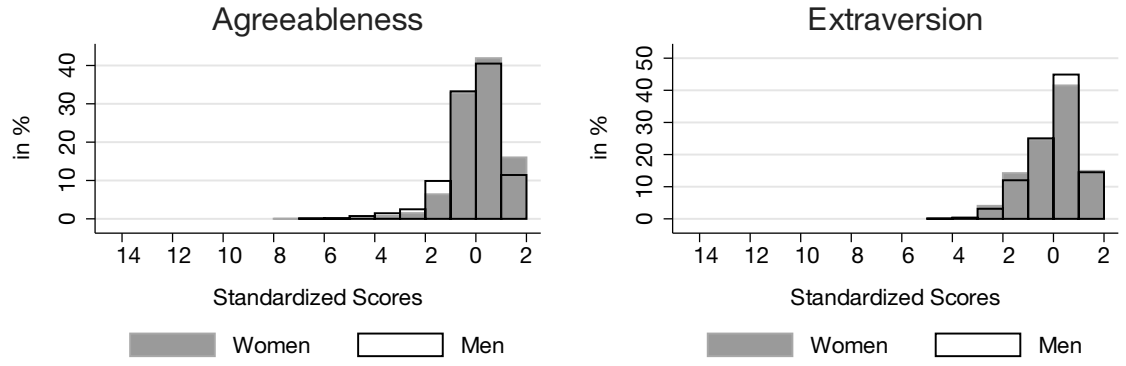

Source: BCS70. Own calculations, $\mathrm{n}=4203$.

0.40, which is lower compared to the finding in Robins et al. (2001) and might be explained by the immense psychological, physical and environmental changes individuals experience in puberty.

Taking a brief look at gender differences, the results show that females score higher in conscientiousness than males at the ages of both 10 and 16, while they score lower in emotional stability at the age of 16 (Figures 3.2 and 3.3). These findings are mainly consistent with Schmitt et al. (2008). Table 3.1 reveals that females more often decrease in emotional stability between the ages of 10 and 16 than males, while males more often increase or stay at the same emotional stability level compared to females. Regarding conscientiousness the changes are less pronounced. ${ }^{14}$

\footnotetext{
14 The discussion of the personality trait measures will be less detailed in the next chapters, because the descriptive statistics are similar throughout the dissertation.
} 
Figure 3.3: Personality Traits at Age 16
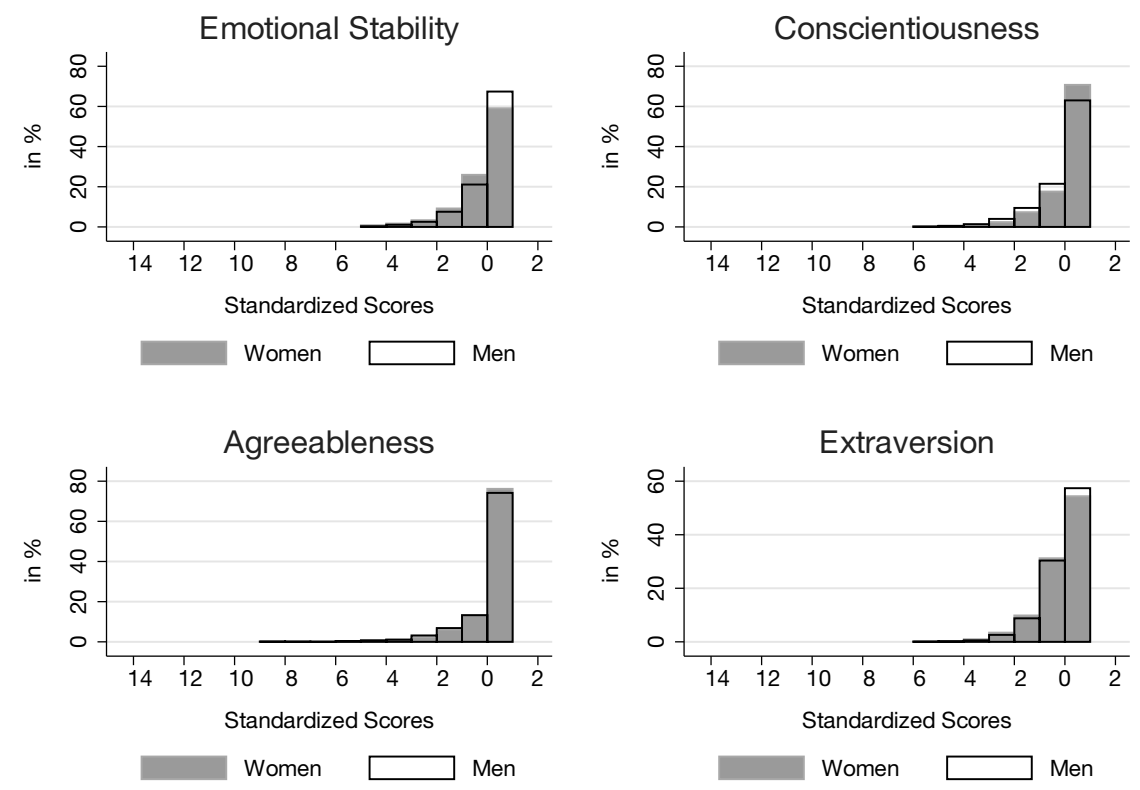

Source: BCS70. Own calculations, $\mathrm{n}=4203$.

Table 3.1: Personality Change Between Ages 10 and 16

\begin{tabular}{llll}
\hline Personality Trait Change & All & Female & Male \\
\hline Increase: Emotional Stability (Age 16-10) $\rangle 0.5$ std & $28 \%$ & $25 \%$ & $32 \%$ \\
Stable: -0.5 std $\leq$ Emotional Stability (Age 16-10) $\leq 0.5$ std & $46 \%$ & $38 \%$ & $47 \%$ \\
Decrease: Emotional Stability (Age 16-10) $<-0.5$ std & $26 \%$ & $30 \%$ & $21 \%$ \\
Increase: Conscientiousness (Age 16-10) $\rangle 0.5$ std & $25 \%$ & $25 \%$ & $28 \%$ \\
Stable: -0.5 std $\leq$ Conscientiousness (Age 16-10) $\leq 0.5$ std & $52 \%$ & $52 \%$ & $49 \%$ \\
Decrease: Conscientiousness (Age 16-10) $<-0.5$ std & $23 \%$ & $23 \%$ & $23 \%$ \\
\hline
\end{tabular}

Source: BCS70, own calculations based on working sample $\mathrm{n}=4203$. 
Appendix Tables 3.11 and 3.12 provide an initial idea about the correlation between personality and educational achievement as well as the distribution of the four personality types and their relation to educational achievement. Appendix Table 3.11 indicates that emotional stability is more correlated with educational achievement for females than for males, while conscientiousness is correlated with education outcomes for both genders. Appendix Table 3.12 shows that females and males have the same share in the groups of more emotionally stable and more conscientious as well as less emotionally stable and less conscientious individuals. Relatively more females are in the group of less emotionally stable, but more conscientious individuals, while relatively more males are in the group of more emotionally stable, but less conscientious individuals. On average, less emotionally stable and less conscientious females have the worst education outcomes, while less emotionally stable and more conscientious males have the highest educational achievement measured in NVQ at the age of 30 .

\subsubsection{Additional Controls}

In addition to the measures discussed above, controls are included for cognitive ability. Following Prevoo and ter Weel (2015), cognitive ability is measured by using test scores at the age of 10 because cognitive ability can be considered as rankorder stable at that age (Lebel and Beaulieu, 2011). Appendix Table 3.13 provides all test scores available at the age of 10 . For the analysis, the results based on the Shortened Edinburgh Reading Test (ERT), the Friendly Maths Test (FMT), the Pictorial Language Comprehension Test (PLCT) and the diagnostic spelling test are selected, because these tests contain the lowest numbers of missing values and measure a broad range of skills. To assess the measure of cognitive ability, the share of correct answers per test is calculated and a standardized score (mean of 0 and standard deviation of 1) out of these four shares by person is derived.

Furthermore, controls are included for the child's family background at birth by including the following variables in all the regressions: mother's age, mother's age squared, parental socioeconomic status and region of living at birth. The variable parental socioeconomic status is a binary variable with value 1 or 0 . The variable is coded as 1 if the highest parental occupation status is non-manual, managerial, technical or professional, whereas it is 0 if the child has a non-working single parent, 
has parents who are unskilled or partly-skilled or the highest occupation status of the parents is manual work.

\section{$3.3 \quad$ Results}

The results are based upon ordinary least squares (OLS) and linear probability regression methods. All regressions focus on the relation between emotional stability and conscientiousness and education outcomes at the age of 30. In all regressions, controls are included for the respondent's personality traits agreeableness and extraversion, region of living at birth as well as the mother's age and mother's age squared. To account for the possible problem of heteroscedasticity, robust standard errors are calculated.

\subsubsection{Personality and Educational Achievement}

Table 3.2 reveals that emotional stability in adolescence is significantly positively related to educational achievement in adult age for women. An increase in emotional stability by 1 standard deviation at the age of 16 is significantly related to an increase of the NVQ level reached by 0.09 , an almost 2 percentage point decrease in the probability of having a poor education qualification and a 2 percentage point increase in the probability of holding an academic degree at the age of 30. However, the emotional stability male interaction terms indicate that emotional stability is less related to educational achievement for men in comparison to women. For men, emotional stability seems to only play a role regarding academic education.

By contrast, conscientiousness in adolescence is positively associated with educational achievement in adulthood for both genders. An increase in conscientiousness of 1 standard deviation at the age of 16 is significantly associated with a boost of the NVQ level reached by 0.15 , a reduced probability of having a poor education qualification by 2.4 percentage points and an almost 5 percentage point higher probability of holding an academic degree at the age of 30 . These results are basically in line with the theoretical considerations, but suggest that low emotional stability particularly constitutes a penalty for women.

Finally, the analysis reveals that both cognitive ability and parental socioeconomic background are significantly positively associated with educational achievement. 
This is a well established result, and therefore, it is not discussed in detail. The size and significance of the related coefficients stays robust in all regressions in this chapters.

Table 3.2: Role of Emotional Stability and Conscientiousness

\begin{tabular}{llll}
\hline Education Level (Age 30) $\ldots$ & NVQ $(0-5)$ & Poor $(0,1)$ & Academic $(4,5)$ \\
\hline Emotional Stability (Age 16) & $0.086^{* *}$ & $-0.019^{*}$ & $0.020^{*}$ \\
& $(0.013)$ & $(0.088)$ & $(0.070)$ \\
Conscientiousness (Age 16) & $0.151^{* * *}$ & $-0.024^{* *}$ & $0.049^{* * *}$ \\
& $(0.000)$ & $(0.030)$ & $(0.000)$ \\
Emotional Stability Male (Age 16) & $-0.134^{* * *}$ & $0.036^{* *}$ & -0.024 \\
& $(0.008)$ & $(0.021)$ & $(0.168)$ \\
Conscientiousness Male (Age 16) & -0.011 & 0.007 & -0.008 \\
& $(0.799)$ & $(0.646)$ & $(0.608)$ \\
Male & $0.133^{* * *}$ & $-0.038^{* * *}$ & 0.021 \\
& $(0.000)$ & $(0.000)$ & $(0.124)$ \\
Cognitive Ability (Age 10) & $0.488^{* * *}$ & $-0.103^{* * *}$ & $0.136^{* * *}$ \\
& $(0.000)$ & $(0.000)$ & $(0.000)$ \\
Higher SES Parents (Birth) & $0.341^{* * *}$ & $-0.048^{* * *}$ & $0.142^{* * *}$ \\
\hline$N$ & $(0.000)$ & $(0.000)$ & $(0.000)$ \\
\hline$R^{2}$ & 4203 & 4203 & 4203 \\
\hline
\end{tabular}

Source: British Cohort Study (BCS70), own calculations.

Note: $p$-values in parentheses ${ }^{*} p<0.10,{ }^{* *} p<0.05,{ }^{* * *} p<0.01$. Poor and academic education are binary variables, whereby the values in brackets are coded as 1 . The regressions include controls for mother's age, mother's age squared, region of living at birth as well as the personality traits agreeableness and extraversion measured at the age of 16 .

In Tables 3.3 and 3.4, the substitution effect of conscientiousness for the relationship between low emotional stability and educational achievement is analyzed. It is hypothesized that at any level of emotional stability, education outcomes are better with increasing conscientiousness. This means that conscientiousness is able to buffer the negative relation between low emotional stability and educational achievement, because it is likely to compensate for the reduced problem-solving ability related to low emotional stability. 
Table 3.3: Substitution Effect of Conscientiousness

\begin{tabular}{llll}
\hline Education Level (Age 30) $\ldots$ & NVQ $(0-5)$ & Poor $(0,1)$ & Academic $(4,5)$ \\
\hline Emotional Stability (Age 16) & $0.097^{* * *}$ & $-0.021^{* *}$ & $0.023^{*}$ \\
& $(0.003)$ & $(0.046)$ & $(0.030)$ \\
Conscientiousness (Age 16) & $0.196^{* * *}$ & $-0.034^{* * *}$ & $0.063^{* * *}$ \\
& $(0.000)$ & $(0.007)$ & $(0.000)$ \\
Emotional Stability Male (Age 16) & $-0.134^{* * *}$ & $0.040^{* * *}$ & -0.019 \\
& $(0.004)$ & $(0.004)$ & $(0.235)$ \\
Conscientiousness Male (Age 16) & -0.045 & 0.016 & -0.017 \\
& $(0.322)$ & $(0.304)$ & $(0.279)$ \\
Emotional Stability $\times$ & $0.056^{* * *}$ & $-0.013^{* *}$ & $0.017^{* * *}$ \\
Conscientiousness (Age 16) & $(0.003)$ & $(0.046)$ & $(0.004)$ \\
Emotional Stability $\times$ & -0.044 & $0.020^{* *}$ & -0.007 \\
Conscientiousness Male (Age 16) & $(0.137)$ & $(0.047)$ & $(0.425)$ \\
Male & $0.160^{* * *}$ & $-0.048^{* * *}$ & $0.027^{*}$ \\
\hline$N$ & $(0.000)$ & $(0.000)$ & $(0.067)$ \\
$R^{2}$ & 4203 & 4203 & 4203 \\
\hline
\end{tabular}

Source: British Cohort Study (BCS70), own calculations.

Note: $p$-values in parentheses ${ }^{*} p<0.10,{ }^{* *} p<0.05,{ }^{* * *} p<0.01$. Poor and academic education are binary variables, whereby the values in brackets are coded as 1 . The regressions include controls for mother's age, mother's age squared, region of living and parental socioeconomic background at birth, the personality traits agreeableness and extraversion measured at the age of 16 as well as cognitive ability measured at the age of 10 .

Table 3.3 shows a significant interaction effect between the continuous variables emotional stability and conscientiousness for women. This means that the positive association between emotional stability and educational achievement is stronger with increasing conscientiousness. Accordingly, conscientiousness is likely to mitigate the negative relationship between low emotional stability and educational achievement which is in line with the theoretical considerations. For men, the substitution effect of conscientiousness only arises for academic education.

In Table 3.4, the substitution effect of conscientiousness is further illuminated by including four personality types comprising (1) more emotionally stable and more conscientious, (2) more emotionally stable and less conscientious, (3) less emotionally stable and more conscientious and (4) less emotionally stable and less conscientious individuals in the regression model. 
Table 3.4: Personality Types and Educational Qualification

\begin{tabular}{llll}
\hline Education Level (Age 30) $\ldots$ & NVQ $(0-5)$ & Poor $(0,1)$ & Academic $(4,5)$ \\
\hline High Emotional Stability, & $-0.357^{* * *}$ & 0.044 & $-0.133^{* * *}$ \\
Low Conscientiousness (Age 16) & $(0.000)$ & $(0.143)$ & $(0.000)$ \\
Low Emotional Stability, & $-0.184^{* * *}$ & 0.022 & $-0.046^{*}$ \\
High Conscientiousness (Age 16) & $(0.009)$ & $(0.261)$ & $(0.071)$ \\
Low Emotional Stability, & $-0.405^{* * *}$ & $0.085^{* * *}$ & $-0.130^{* * *}$ \\
Low Conscientiousness (Age 16) & $(0.000)$ & $(0.001)$ & $(0.000)$ \\
High Emotional Stability, & 0.120 & -0.030 & 0.043 \\
Low Conscientiousness Male (Age 16) & $(0.329)$ & $(0.436)$ & $(0.305)$ \\
Low Emotional Stability, & $0.310^{* * *}$ & $-0.053^{*}$ & 0.060 \\
High Conscientiousness Male (Age 16) & $(0.005)$ & $(0.075)$ & $(0.159)$ \\
Low Emotional Stability, & 0.184 & $-0.093^{* *}$ & 0.036 \\
Low Conscientiousness Male (Age 16) & $(0.113)$ & $(0.011)$ & $(0.368)$ \\
Male & 0.032 & -0.006 & 0.001 \\
\hline$N$ & $(0.569)$ & $(0.684)$ & $(0.969)$ \\
$R^{2}$ & 4203 & 4203 & 4203 \\
\hline
\end{tabular}

Source: British Cohort Study (BCS70), own calculations.

Note: $p$-values in parentheses ${ }^{*} p<0.10,{ }^{* *} p<0.05,{ }^{* * *} p<0.01$. Poor and academic education are binary variables, whereby the values in brackets are coded as 1 . The reference group for the personality types comprises emotionally stable and highly conscientious individuals. The regressions include controls for mother's age, mother's age squared, region of living and parental socioeconomic background at birth, the personality traits agreeableness and extraversion measured at the age of 16 as well as cognitive ability measured at the age of 10 .

For women, Table 3.4 suggests that scoring both low in emotional stability and conscientiousness at the age of 16 is negatively related to educational achievement at the age of 30. Low emotionally stable and low conscientious women score 0.41 lower in the NVQ level, have a 8.5 percentage points higher probability of having no or a very limited education qualification and have a 13 percentage points lower probability of holding an academic degree at the age of 30 compared to the reference group. Higher conscientiousness significantly substitutes for low emotional stability.

Moreover, the risk of poor educational achievement at the age of 30 is not significantly different for more emotionally stable, but less conscientious women compared to the reference group. This suggests that more emotional stability is meaningful to overcome the risk of poor education achievement at the age of 30, which might 
be dedicated to a lower stress-reactivity of emotionally stable women. However, for average educational achievement or academic achievement, conscientiousness seems to be crucial.

In contrast, low emotionally stable and low conscientious men have a 9 percentage point lower risk to only reach poor education outcomes at the age of 30 compared to women with the same characteristics. For academic education, particularly low conscientiousness constitutes a penalty for both women and men by decreasing the probability of holding an academic degree at the age of 30 by 13 percentage points.

Interestingly, low emotionally stable, but more conscientious men have significantly better education outcomes than women with the same characteristics. However, their education outcomes are as well slightly better than those for emotionally stable and more conscientious men, i.e. theoretically considered as the most favorable group (see also Appendix Table 3.12). This suggests that men might even profit from low emotional stability - at least if low emotional stability is combined with more conscientiousness.

Finally, Table 3.2 reveals that men have significantly improved education outcomes compared to women at the age of 30. In Table 3.4, both size and significance of these coefficients have vanished. This result is further investigated and discussed in Chapter 4.

\subsubsection{Personality Change and Educational Achievement}

Table 3.5 shows that emotional stability at the age of 10 is positively related to educational achievement at the age of 30 for women. A 1 standard deviation higher level of emotional stability is (borderline) significantly associated with a 0.10 higher NVQ level, an almost 2 percentage points lower probability of having no or a limited education qualification and a 2.4 percentage points higher probability of holding an academic degree at the age of 30 . 
Table 3.5: Personality Change and Educational Qualification

\begin{tabular}{|c|c|c|c|}
\hline Education Level (Age 30) ... & NVQ $(0-5)$ & Poor $(0,1)$ & Academic $(4,5)$ \\
\hline Emotional Stability (Age 10) & $\begin{array}{l}0.102^{* *} \\
(0.012)\end{array}$ & $\begin{array}{l}-0.019 \\
(0.148)\end{array}$ & $\begin{array}{l}0.024^{*} \\
(0.064)\end{array}$ \\
\hline Conscientiousness (Age 10) & $\begin{array}{l}0.131^{* * *} \\
(0.001)\end{array}$ & $\begin{array}{c}-0.028^{*} \\
(0.051)\end{array}$ & $\begin{array}{l}0.034^{* * *} \\
(0.010)\end{array}$ \\
\hline Emotional Stability Male (Age 10) & $\begin{array}{l}-0.159^{* * *} \\
(0.006)\end{array}$ & $\begin{array}{l}0.049^{* * *} \\
(0.008)\end{array}$ & $\begin{array}{l}-0.026 \\
(0.175)\end{array}$ \\
\hline Conscientiousness Male (Age 10) & $\begin{array}{l}-0.059 \\
(0.273)\end{array}$ & $\begin{array}{l}0.021 \\
(0.238)\end{array}$ & $\begin{array}{l}-0.017 \\
(0.354)\end{array}$ \\
\hline Emotional Stability (Age 16-10) $>0.5$ & $\begin{array}{l}0.135^{*} \\
(0.086)\end{array}$ & $\begin{array}{c}-0.030 \\
(0.191)\end{array}$ & $\begin{array}{l}0.019 \\
(0.501)\end{array}$ \\
\hline Emotional Stability (Age 16-10) $<-0.5$ & $\begin{array}{l}-0.032 \\
(0.623)\end{array}$ & $\begin{array}{l}-0.002 \\
(0.898)\end{array}$ & $\begin{array}{l}-0.020 \\
(0.404)\end{array}$ \\
\hline Conscientiousness (Age 16-10) $>0.5$ & $\begin{array}{l}0.021 \\
(0.811)\end{array}$ & $\begin{array}{l}0.011 \\
(0.680)\end{array}$ & $\begin{array}{l}-0.002 \\
(0.947)\end{array}$ \\
\hline Conscientiousness (Age 16-10) $<-0.5$ & $\begin{array}{l}-0.322^{* * *} \\
(0.000)\end{array}$ & $\begin{array}{l}0.069^{* * *} \\
(0.002)\end{array}$ & $\begin{array}{l}-0.109^{* * *} \\
(0.000)\end{array}$ \\
\hline Emotional Stability Male (Age 16-10) $>0.5$ & $\begin{array}{l}-0.222^{* *} \\
(0.043)\end{array}$ & $\begin{array}{l}0.053 \\
(0.106)\end{array}$ & $\begin{array}{l}-0.039 \\
(0.322)\end{array}$ \\
\hline Emotional Stability Male (Age 16-10) $<-0.5$ & $\begin{array}{l}0.096 \\
(0.333)\end{array}$ & $\begin{array}{l}-0.040 \\
(0.169)\end{array}$ & $\begin{array}{l}0.009 \\
(0.799)\end{array}$ \\
\hline Conscientiousness Male (Age 16-10) $>0.5$ & $\begin{array}{l}0.024 \\
(0.836)\end{array}$ & $\begin{array}{l}-0.030 \\
(0.405)\end{array}$ & $\begin{array}{l}0.004 \\
(0.925)\end{array}$ \\
\hline Conscientiousness Male (Age 16-10) $<-0.5$ & $\begin{array}{l}0.100 \\
(0.322)\end{array}$ & $\begin{array}{l}-0.040 \\
(0.192)\end{array}$ & $\begin{array}{l}0.037 \\
(0.292)\end{array}$ \\
\hline Male & $\begin{array}{l}0.085 \\
(0.328)\end{array}$ & $\begin{array}{l}-0.031 \\
(0.214)\end{array}$ & $\begin{array}{c}-0.020 \\
(0.528)\end{array}$ \\
\hline$N$ & 4203 & 4203 & 4203 \\
\hline$R^{2}$ & 0.252 & 0.131 & 0.186 \\
\hline
\end{tabular}

Source: British Cohort Study (BCS70), own calculations.

Note: $p$-values in parentheses ${ }^{*} p<0.10,{ }^{* *} p<0.05,{ }^{* * *} p<0.01$. Poor and academic education are binary variables, whereby the values in brackets are coded as 1 . The OLS regressions include controls for mother's age, mother's age squared, region of living and parental socioeconomic background at birth, the personality traits agreeableness and extraversion measured at the ages of 10 and 16 as well as cognitive ability measured at the age of 10 . 
Furthermore, an increase in emotional stability of 0.5 standard deviations or more between the ages of 10 and 16 is (borderline) significantly related to a 0.14 higher NVQ level and a 3 percentage points lower probability of having a poor education outcome at the age of 30 . Moreover, the male interaction terms again suggest that low emotional stability is significantly less important for men compared to women when it comes to educational achievement.

By contrast, conscientiousness plays a role for both genders regarding educational achievement. A 1 standard deviation higher level of conscientiousness at the age of 10 is significantly associated with a boost of the NVQ level reached by 0.13 , a 2.8 lower probability of having no or a limited education qualification and a 3.4 percentage points higher probability of holding an academic degree at the age of 30 . A drop in conscientiousness of 0.5 standard deviations or more between the ages of 10 and 16 is significantly related to a reduction of the NVQ level reached by 0.32 , an almost 7 percentage points increase in having a poor education qualification and a 11 percentage points lower probability of holding an academic degree at the age of 30 .

\subsection{Conclusion}

The empirical analysis tests the economic framework presented in Chapter 2 by focusing on an objective measure of educational achievement at the age of 30 . It analyzes the predictive power of (1) low emotional stability at the age of 16 for educational achievement at the age of 30 as well as the possible substitution effect of conscientiousness and (2) a change in emotional stability and conscientiousness between the ages of 10 and 16 and educational achievement at the age of 30 .

The main results are summarized as follows: First, the results reveal that emotional stability predicts educational achievement for women. Women who score low in both emotional stability and conscientiousness at the age of 16 have the worst educational achievement at the age of 30. For instance, low emotionally stable and low conscientious women have an 8.5 percentage points higher risk to reach no or very limited education at the age of 30 compared to more emotionally stable and more conscientious women. For men, emotional stability seems to be less of concern. Moreover, the analysis shows that conscientiousness is an important predictor for educational achievement for both women and men. Accordingly, more conscientiousness at the 
age of 16 significantly boosts educational achievement.

Second, an increase in emotional stability between the ages of 10 and 16 is significantly associated with improved educational achievement for women. Both females and males who decrease in conscientiousness between the ages of 10 and 16 have a significantly lower educational achievement at the age of 30. These findings emphasize the importance of personality development for socioeconomic success in later life (see Chapter 5). Taking into account that the regressions control for cognitive ability at the age of 10 , the results are not only statistically significant, but also economically meaningful.

For women, the results regarding the association between emotional stability and educational achievement are in line with the theoretical considerations. Low emotional stability is likely to be related to arousal and procrastination or avoidance temperament. This is likely to be an important channel for worse experiences in school and earlier school dropout (De Paola and Scoppa, 2015). Moreover, the findings emphasize the importance of conscientiousness for educational achievement independent of gender. This is likely to be related to improved problem-solving skills and more self-discipline in challenging situations (Johnson and Bloom, 1995). The economic framework is further tested in the next chapter. 


\subsection{Appendix}

\subsubsection{Data Description}

Table 3.6: Number of Observations by Wave and Variables Used

\begin{tabular}{|c|c|c|c|}
\hline Wave; Year & $\mathrm{N}$ & Dataset & Variables \\
\hline $\begin{array}{l}1 ; \\
1970\end{array}$ & 17,196 & SN2666: Chamberlain et al. (2013) & $\begin{array}{l}\text { Mother's age at birth, region of } \\
\text { birth, child's gender, parental so- } \\
\text { cioeconomic status at birth (all } \\
\text { chapters). }\end{array}$ \\
\hline $\begin{array}{l}3 ; \\
1980\end{array}$ & 14,875 & SN3723: Butler et al. (2014) & $\begin{array}{l}\text { Test scores and personality traits at } \\
\text { the age of } 10 \text { (all chapters). }\end{array}$ \\
\hline $\begin{array}{l}4 ; \\
1986\end{array}$ & 11,615 & SN3535: Butler et al. (2013) & $\begin{array}{l}\text { Personality traits (all chapters), in- } \\
\text { dicator about pessimistic expecta- } \\
\text { tions with regard to exams (Chap- } \\
\text { ter } 4 \text { ), indicators of helplessness and } \\
\text { hopelessness (Chapter } 6 \text { ) and mental } \\
\text { ill-health (Chapters } 5 \text { and } 6 \text { ) at the } \\
\text { age of } 16 \text {. }\end{array}$ \\
\hline $\begin{array}{l}5 ; \\
1996\end{array}$ & 9,003 & $\begin{array}{l}\text { SN3833: Bynner and University } \\
\text { of London, Institute of Educa- } \\
\text { tion, Centre for Longitudinal Studies } \\
(2013)\end{array}$ & $\begin{array}{l}\text { Mental ill-health at the age of } 26 \\
\text { (Chapters } 5 \text { and } 6 \text { ). }\end{array}$ \\
\hline $\begin{array}{l}6 ; \\
2000\end{array}$ & 11,261 & $\begin{array}{l}\text { SN5558: University of London, Insti- } \\
\text { tute of Education, Centre for Longi- } \\
\text { tudinal Studies (2013b) }\end{array}$ & $\begin{array}{l}\text { Education achievement (Chapters } 3 \\
\text { and } 4 \text { ), mental ill-health (Chapters } 5 \\
\text { and } 6 \text { ) and indicator of helplessness } \\
\text { (Chapter 6) at the age of } 30 .\end{array}$ \\
\hline $\begin{array}{l}7 \\
2004\end{array}$ & 9,665 & $\begin{array}{l}\text { SN5585: University of London, Insti- } \\
\text { tute of Education, Centre for Longi- } \\
\text { tudinal Studies (2013a) }\end{array}$ & $\begin{array}{l}\text { Mental ill-health at the age of } 34 \\
\text { (Chapters } 5 \text { and } 6 \text { ). }\end{array}$ \\
\hline $\begin{array}{l}9 ; \\
2012\end{array}$ & 9,841 & $\begin{array}{l}\text { SN7473: University of London. Insti- } \\
\text { tute of Education. Centre for Longi- } \\
\text { tudinal Studies }(2012)\end{array}$ & $\begin{array}{l}\text { Mental ill-health at the age of } 42 \\
\text { (Chapters } 5 \text { and } 6 \text { ). }\end{array}$ \\
\hline
\end{tabular}


Table 3.7: Descriptive Statistics

\begin{tabular}{|c|c|c|c|c|c|c|c|c|c|c|}
\hline \multirow[b]{2}{*}{ Variable Names } & \multicolumn{5}{|c|}{ Working Sample } & \multicolumn{5}{|c|}{ Unrestricted Sample } \\
\hline & $\mathrm{N}$ & Mean & SD & Min & $\operatorname{Max}$ & $\mathrm{N}$ & Mean & SD & Min & $\operatorname{Max}$ \\
\hline Highest NVQ Level (Age 30) & 4203 & 2.69 & 1.38 & 0.00 & 5.00 & 7704 & 2.56 & 1.42 & 0.00 & 5.00 \\
\hline No Qualifications (Age 30) & 4203 & 0.17 & 0.38 & 0.00 & 1.00 & 7704 & 0.20 & 0.40 & 0.00 & 1.00 \\
\hline Academic Degree (Age 30) & 4203 & 0.37 & 0.48 & 0.00 & 1.00 & 7704 & 0.34 & 0.48 & 0.00 & 1.00 \\
\hline Emotional Stability (ES, Age 10) & 4203 & 0.07 & 0.94 & -4.12 & 1.33 & 8910 & 0.00 & 1.00 & -4.28 & 1.33 \\
\hline Conscientiousness (C, Age 10) & 4203 & 0.09 & 0.94 & -3.41 & 1.20 & 8910 & 0.00 & 1.00 & -3.45 & 1.20 \\
\hline Agreeableness (A, Age 10) & 4203 & 0.08 & 0.91 & -6.64 & 1.34 & 8910 & 0.00 & 1.00 & -6.64 & 1.34 \\
\hline Extraversion (E, Age 10) & 4203 & 0.03 & 0.97 & -4.37 & 1.61 & 8910 & -0.00 & 1.00 & -4.37 & 1.61 \\
\hline Emotional Stability (ES, Age 16) & 4203 & 0.06 & 0.95 & -4.60 & 0.85 & 6768 & 0.00 & 1.00 & -4.60 & 0.85 \\
\hline Conscientiousness (C, Age 16) & 4203 & 0.06 & 0.94 & -4.76 & 0.71 & 6768 & -0.00 & 1.00 & -4.76 & 0.71 \\
\hline Agreeableness (A, Age 16) & 4203 & 0.07 & 0.90 & -7.69 & 0.52 & 6768 & 0.00 & 1.00 & -8.29 & 0.52 \\
\hline Extraversion (E, Age 16) & 4203 & 0.03 & 0.96 & -5.49 & 0.98 & 6768 & 0.00 & 1.00 & -5.49 & 0.98 \\
\hline High ES, High C (Age 16) & 4203 & 0.50 & 0.50 & 0.00 & 1.00 & 6768 & 0.48 & 0.50 & 0.00 & 1.00 \\
\hline High ES, Low C (Age 16) & 4203 & 0.13 & 0.34 & 0.00 & 1.00 & 6768 & 0.13 & 0.34 & 0.00 & 1.00 \\
\hline Low ES, High C (Age 16) & 4203 & 0.17 & 0.38 & 0.00 & 1.00 & 6768 & 0.17 & 0.38 & 0.00 & 1.00 \\
\hline Low ES, Low C (Age 16) & 4203 & 0.20 & 0.40 & 0.00 & 1.00 & 6768 & 0.22 & 0.41 & 0.00 & 1.00 \\
\hline Male & 4203 & 0.46 & 0.50 & 0.00 & 1.00 & 10046 & 0.50 & 0.50 & 0.00 & 1.00 \\
\hline Cognitive Ability (Age 10) & 4203 & 0.15 & 0.93 & -3.51 & 2.18 & 8586 & -0.00 & 1.00 & -4.10 & 2.18 \\
\hline Higher SES Parents (Birth) & 4203 & 0.20 & 0.40 & 0.00 & 1.00 & 10033 & 0.19 & 0.39 & 0.00 & 1.00 \\
\hline Region (Birth) & 4203 & 5.66 & 2.71 & 1.00 & 10.00 & 10046 & 5.69 & 2.64 & 1.00 & 11.00 \\
\hline Age Mother (Birth) & 4203 & 22.64 & 3.94 & 14.00 & 46.00 & 9989 & 22.33 & 3.96 & 13.00 & 47.00 \\
\hline
\end{tabular}

Source: BCS70, own calculations. SES refers to the term socioeconomic status. 
Table 3.8: Orthogonal Principle Component Factoring at Age 10

\begin{tabular}{|c|c|c|c|c|c|}
\hline Items & ES & $\mathrm{CO}$ & AG & EX & Uniqueness \\
\hline R8. Irritable. & 0.752 & & & & 0.396 \\
\hline R9. Miserable and tearful. & 0.444 & & 0.264 & 0.363 & 0.590 \\
\hline C8. Requests must be met. & 0.658 & 0.209 & & & 0.483 \\
\hline C12. Sullen or sulky. & 0.598 & & & & 0.568 \\
\hline C16. Changes mood quickly. & 0.742 & 0.208 & & & 0.373 \\
\hline C17. Temper unpredictable. & 0.757 & & 0.214 & & 0.361 \\
\hline R15. Cannot settle. & & 0.745 & & & 0.387 \\
\hline C3. Easily distracted. & & 0.754 & & & 0.375 \\
\hline C13. Fails to finish things. & & 0.832 & & & 0.273 \\
\hline C19. Difficulty concentrating. & & 0.778 & & & 0.345 \\
\hline R3. Destroys belongings. & & & 0.675 & & 0.493 \\
\hline R4. Frequently fights. & 0.254 & & 0.613 & & 0.544 \\
\hline R10. Takes others' belongings. & & & 0.715 & & 0.454 \\
\hline R14. Often disobedient. & 0.468 & 0.278 & 0.407 & & 0.536 \\
\hline R18. Often tells lies. & 0.213 & 0.219 & 0.658 & & 0.474 \\
\hline R19. Bullies other children. & 0.230 & & 0.690 & & 0.452 \\
\hline C11. Interferes with others. & 0.282 & 0.283 & 0.513 & & 0.571 \\
\hline R5. Not much liked by others. & & & 0.424 & 0.285 & 0.701 \\
\hline R6. Often worried. & & & & 0.745 & 0.392 \\
\hline R7. Rather solitary. & & & & 0.490 & 0.728 \\
\hline R16. Afraid of new things. & & & & 0.714 & 0.457 \\
\hline R17. Fussy or over-particular. & 0.245 & & & 0.491 & 0.682 \\
\hline
\end{tabular}

Source: BCS70, own calculations based on working sample $\mathrm{n}=4203$.

Note: Items are stated in original terms, but reversely used in the analysis. ES = Emotional Stability, $\mathrm{CO}=$ Conscientiousness, $\mathrm{AG}=$ Agreeableness, $\mathrm{EX}=$ Extraversion. Only loadings larger than 0.2 are reported. 
Table 3.9: Orthogonal Principle Component Factoring at Age 16

\begin{tabular}{|c|c|c|c|c|c|}
\hline Items & ES & $\mathrm{CO}$ & AG & EX & Uniqueness \\
\hline R8. Irritable. & 0.746 & & & & 0.388 \\
\hline R9. Miserable and tearful. & 0.469 & & 0.213 & 0.387 & 0.584 \\
\hline C8. Requests must be met. & 0.669 & 0.289 & & & 0.448 \\
\hline C12. Sullen or sulky. & 0.629 & & & & 0.539 \\
\hline C16. Changes mood quickly. & 0.772 & 0.243 & & & 0.325 \\
\hline C17. Temper unpredictable. & 0.760 & 0.204 & & & 0.348 \\
\hline R15. Cannot settle. & & 0.681 & 0.236 & & 0.445 \\
\hline C3. Easily distracted. & 0.246 & 0.750 & & & 0.365 \\
\hline C13. Fails to finish things. & 0.206 & 0.806 & & & 0.297 \\
\hline C19. Difficulty concentrating. & & 0.776 & & & 0.365 \\
\hline R3. Destroys belongings. & & & 0.649 & & 0.528 \\
\hline R4. Frequently fights. & 0.343 & & 0.564 & & 0.564 \\
\hline R10. Takes others' belongings. & & 0.215 & 0.667 & & 0.491 \\
\hline R14. Often disobedient. & 0.454 & 0.302 & 0.448 & & 0.500 \\
\hline R18. Often tells lies. & 0.247 & 0.295 & 0.593 & & 0.500 \\
\hline R19. Bullies other children. & 0.285 & & 0.571 & & 0.590 \\
\hline C11. Interferes with others. & 0.416 & 0.282 & 0.307 & & 0.654 \\
\hline R5. Not much liked by others. & & & 0.494 & 0.332 & 0.640 \\
\hline R6. Often worried. & & & & 0.720 & 0.430 \\
\hline R7. Rather solitary. & & & & 0.554 & 0.650 \\
\hline R16. Afraid of new things. & & & & 0.688 & 0.488 \\
\hline R17. Fussy or over-particular. & 0.293 & & & 0.419 & 0.730 \\
\hline
\end{tabular}

Source: BCS70, own calculations based on working sample $\mathrm{n}=4203$.

Note: Items are stated in original terms, but reversely used in the analysis. ES = Emotional Stability, $\mathrm{CO}=$ Conscientiousness, $\mathrm{AG}=$ Agreeableness, $\mathrm{EX}=$ Extraversion. Only loadings larger than 0.2 are reported. 
Table 3.10: Personality Traits at the Ages of 10 and 16

\begin{tabular}{|c|c|c|c|c|}
\hline $\begin{array}{l}\text { Personality } \\
\text { Trait }\end{array}$ & $\begin{array}{l}\text { Correlation } \\
80-86\end{array}$ & $\begin{array}{l}\text { Explained } \\
\text { Variance }\end{array}$ & $\begin{array}{l}\text { Cronbach's } \\
\text { Alpha }\end{array}$ & Items \\
\hline \multicolumn{5}{|c|}{ Emotional Stability } \\
\hline Age 10 & & $52.90 \%$ & 0.82 & R8. Irritable. \\
\hline Age 16 & 0.40 & $55.40 \%$ & 0.84 & $\begin{array}{l}\text { R9. Miserable and tearful. } \\
\text { C8. Requests must be met. } \\
\text { C12. Sullen or sulky. } \\
\text { C16. Changes mood quickly. } \\
\text { C17. Temper unpredictable. }\end{array}$ \\
\hline \multicolumn{5}{|c|}{ Conscientiousness } \\
\hline Age 10 & & $66.08 \%$ & 0.83 & R15. Cannot settle. \\
\hline Age 16 & 0.42 & $63.85 \%$ & 0.81 & $\begin{array}{l}\text { C3. Easily distracted. } \\
\text { C13. Fails to finish things. } \\
\text { C19. Difficulty concentrating. }\end{array}$ \\
\hline \multicolumn{5}{|c|}{ Agreeableness } \\
\hline Age 10 & & $48.04 \%$ & 0.82 & R3. Destroys belongings. \\
\hline Age 16 & 0.35 & $42.32 \%$ & 0.77 & $\begin{array}{l}\text { R4. Frequently fights. } \\
\text { R10. Takes others' belongings. } \\
\text { R14. Often disobedient. } \\
\text { R18. Often tells lies. } \\
\text { R19. Bullies other children. } \\
\text { C11. Interferes with others. }\end{array}$ \\
\hline \multicolumn{5}{|l|}{ Extraversion } \\
\hline Age 10 & & $37.13 \%$ & 0.56 & R5. Not much liked by others. \\
\hline Age 16 & 0.34 & $36.28 \%$ & 0.57 & $\begin{array}{l}\text { R6. Often worried. } \\
\text { R7. Rather solitary. } \\
\text { R16. Afraid of new things. } \\
\text { R17. Fussy or over-particular. }\end{array}$ \\
\hline
\end{tabular}

Source: BCS70, own calculations based on working sample $\mathrm{n}=4203$.

Note: Items are stated in original terms, but reversely used in the analysis. 
Table 3.11: Correlation between Personality and Educational Qualification

\begin{tabular}{lllllll}
\hline & $\begin{array}{l}\text { Female } \\
\text { NVQ }\end{array}$ & Poor & Academic & $\begin{array}{l}\text { Male } \\
\text { NVQ }\end{array}$ & Poor & Academic \\
\hline Emotional Stability & & & & & & \\
Age 10 & .15 & -.11 & .12 & .10 & -.04 & .11 \\
Age 16 & .18 & -.12 & .09 & .07 & - & .09 \\
Conscientiousness & & & & & & \\
Age 10 & .20 & -.15 & .16 & .20 & -.12 & .17 \\
Age 16 & .23 & -.15 & .20 & .22 & -.13 & .19 \\
\hline
\end{tabular}

Source: BCS70, own calculations based on working sample $\mathrm{n}=4203$.

Note: Correlation coefficients reported are significant at the $5 \%$ level or higher. NVQ = National Vocational Qualification (Scale 0-5), Poor Education Qualification (Binary: 1=0,1), Academic Qualification (Binary: $1=4,5$ ) at the age of 30 .

Table 3.12: Personality Types and Mean Educational Qualification

\begin{tabular}{|c|c|c|c|c|c|c|}
\hline Personality Type & $\begin{array}{l}\text { All } \\
\text { Share }\end{array}$ & $\varnothing \mathrm{NVQ}$ & $\begin{array}{l}\text { Female } \\
\text { Share }\end{array}$ & $\varnothing \mathrm{NVQ}$ & $\begin{array}{l}\text { Male } \\
\text { Share }\end{array}$ & $\varnothing \mathrm{NVQ}$ \\
\hline $\begin{array}{l}\text { High Emotional Stability, } \\
\text { High Conscientiousness }\end{array}$ & $50 \%$ & 2.92 & $50 \%$ & 2.93 & $50 \%$ & 2.91 \\
\hline $\begin{array}{l}\text { High Emotional Stability, } \\
\text { Low Conscientiousness }\end{array}$ & $13 \%$ & 2.35 & $9 \%$ & 2.32 & $17 \%$ & 2.37 \\
\hline $\begin{array}{l}\text { Low Emotional Stability, } \\
\text { High Conscientiousness }\end{array}$ & $17 \%$ & 2.76 & $21 \%$ & 2.62 & $13 \%$ & 3.02 \\
\hline $\begin{array}{l}\text { Low Emotional Stability, } \\
\text { Low Conscientiousness }\end{array}$ & $20 \%$ & 2.25 & $20 \%$ & 2.16 & $20 \%$ & 2.35 \\
\hline Total & $100 \%$ & 2.69 & $100 \%$ & 2.65 & $100 \%$ & 2.72 \\
\hline
\end{tabular}

Source: BCS70, own calculations based on working sample $\mathrm{n}=4203$.

Note: Column percentages by gender are shown. NVQ = National Vocational Qualification (Scale $0-5)$. 
3 PERSONALITY AND EDUCATIONAL ACHIEVEMENT

Table 3.13: Cognitive Ability Measures at the Age of 10

\begin{tabular}{|c|c|}
\hline Educational Test & Components \\
\hline British Abilities Scale (BAS) & $\begin{array}{l}\text { Self-completion; Non-verbal: Recall of dig- } \\
\text { its ( } 34 \text { items), matrices ( } 28 \text { items); Verbal: } \\
\text { Word definitions ( } 37 \text { items), word similari- } \\
\text { ties ( } 42 \text { items). }\end{array}$ \\
\hline Shortened Edinburgh Reading Test (ERT) & Self-completion (64 items). \\
\hline Friendly Maths Test (FMT) & 72 multiple choice questions. \\
\hline Pictorial Language Comprehension Test (PLCT) & $\begin{array}{l}\text { Vocabulary items, sequencing items, sen- } \\
\text { tence comprehension items ( } 100 \text { items). }\end{array}$ \\
\hline Diagnostic Test & $\begin{array}{l}\text { Reading (108 items) and spelling test ( } 50 \\
\text { items). }\end{array}$ \\
\hline
\end{tabular}

Source: BCS70, SN3723: Butler et al. (2014). 


\subsubsection{Robustness Checks}

\subsubsection{Imputation of Missing Values}

Table 3.14: Role of Emotional Stability and Conscientiousness

\begin{tabular}{|c|c|c|c|}
\hline Education Level (Age 30) ... & NVQ $(0-5)$ & Poor $(0,1)$ & Academic $(4,5)$ \\
\hline Emotional Stability (Age 16) & $\begin{array}{l}0.076^{* *} \\
(0.021)\end{array}$ & $\begin{array}{l}-0.015 \\
(0.144)\end{array}$ & $\begin{array}{l}0.022^{* *} \\
(0.039)\end{array}$ \\
\hline Conscientiousness (Age 16) & $\begin{array}{l}0.159^{* * *} \\
(0.000)\end{array}$ & $\begin{array}{l}-0.030^{* * *} \\
(0.004)\end{array}$ & $\begin{array}{l}0.051^{* * *} \\
(0.000)\end{array}$ \\
\hline Emotional Stability Male (Age 16) & $\begin{array}{l}-0.104^{* *} \\
(0.031)\end{array}$ & $\begin{array}{l}0.028^{*} \\
(0.072)\end{array}$ & $\begin{array}{l}-0.022 \\
(0.172)\end{array}$ \\
\hline Conscientiousness Male (Age 16) & $\begin{array}{l}0.001 \\
(0.979)\end{array}$ & $\begin{array}{l}0.006 \\
(0.659)\end{array}$ & $\begin{array}{l}-0.002 \\
(0.880)\end{array}$ \\
\hline Male & $\begin{array}{l}0.136^{* * *} \\
(0.000)\end{array}$ & $\begin{array}{l}-0.038^{* * *} \\
(0.000)\end{array}$ & $\begin{array}{l}0.019 \\
(0.141)\end{array}$ \\
\hline Cognitive Ability (Age 10) & $\begin{array}{l}0.494^{* * *} \\
(0.000)\end{array}$ & $\begin{array}{l}-0.106^{* * *} \\
(0.000)\end{array}$ & $\begin{array}{l}0.136^{* * *} \\
(0.000)\end{array}$ \\
\hline Higher SES Parents (Birth) & $\begin{array}{l}0.372^{* * *} \\
(0.000)\end{array}$ & $\begin{array}{l}-0.057^{* * *} \\
(0.000)\end{array}$ & $\begin{array}{l}0.142^{* * *} \\
(0.000)\end{array}$ \\
\hline $\begin{array}{l}N \\
R^{2}\end{array}$ & $\begin{array}{l}4908 \\
0.251\end{array}$ & $\begin{array}{l}4908 \\
0.130\end{array}$ & $\begin{array}{l}4908 \\
0.185\end{array}$ \\
\hline
\end{tabular}

Source: British Cohort Study (BCS70), own calculations.

Note: $p$-values in parentheses ${ }^{*} p<0.10,{ }^{* *} p<0.05,{ }^{* * *} p<0.01$. Poor and academic education are binary variables, whereby the values in brackets are coded as 1 . The regressions include controls for mother's age, mother's age squared, region of living at birth as well as the personality traits agreeableness and extraversion measured at the age of 16 . 
Table 3.15: Substitution Effect of Conscientiousness

\begin{tabular}{llll}
\hline Education Level (Age 30) $\ldots$ & NVQ $(0-5)$ & Poor $(0,1)$ & Academic $(4,5)$ \\
\hline Emotional Stability (Age 16) & $0.083^{* * *}$ & -0.013 & $0.024^{* *}$ \\
& $(0.008)$ & $(0.168)$ & $(0.017)$ \\
Conscientiousness (Age 16) & $0.189^{* * *}$ & $-0.034^{* * *}$ & $0.059^{* * *}$ \\
& $(0.000)$ & $(0.004)$ & $(0.000)$ \\
Emotional Stability Male (Age 16) & $-0.096^{* *}$ & $0.025^{*}$ & -0.014 \\
& $(0.028)$ & $(0.058)$ & $(0.364)$ \\
Conscientiousness Male (Age 16) & -0.015 & 0.008 & -0.004 \\
& $(0.728)$ & $(0.567)$ & $(0.754)$ \\
Emotional Stability $\times$ & $0.040^{* *}$ & -0.008 & $0.011^{* *}$ \\
Conscientiousness (Age 16) & $(0.027)$ & $(0.218)$ & $(0.049)$ \\
Emotional Stability $\times$ & -0.016 & 0.011 & -0.003 \\
Conscientiousness Male (Age 16) & $(0.561)$ & $(0.243)$ & $(0.704)$ \\
Male & $0.147^{* * *}$ & $-0.043^{* * *}$ & 0.018 \\
& $(0.000)$ & $(0.000)$ & $(0.176)$ \\
\hline$N$ & 4908 & 4908 & 4908 \\
$R^{2}$ & 0.251 & 0.129 & 0.186 \\
\hline
\end{tabular}

Source: British Cohort Study (BCS70), own calculations.

Note: $p$-values in parentheses ${ }^{*} p<0.10,{ }^{* *} p<0.05,{ }^{* *} p<0.01$. Poor and academic education are binary variables, whereby the values in brackets are coded as 1 . The regressions include controls for mother's age, mother's age squared, region of living and parental socioeconomic background at birth, the personality traits agreeableness and extraversion measured at the age of 16 as well as cognitive ability measured at the age of 10 . 
Table 3.16: Personality Types and Educational Qualification

\begin{tabular}{llll}
\hline Education Level (Age 30) ... & NVQ (0-5) & Poor $(0,1)$ & Academic $(4,5)$ \\
\hline High Emotional Stability, & $-0.343^{* * *}$ & $0.053^{*}$ & $-0.113^{* * *}$ \\
Low Conscientiousness (Age 16) & $(0.000)$ & $(0.063)$ & $(0.000)$ \\
Low Emotional Stability, & $-0.136^{* *}$ & 0.016 & -0.034 \\
High Conscientiousness (Age 16) & $(0.037)$ & $(0.407)$ & $(0.143)$ \\
Low Emotional Stability, & $-0.390^{* * *}$ & $0.082^{* * *}$ & $-0.125^{* * *}$ \\
Low Conscientiousness (Age 16) & $(0.000)$ & $(0.001)$ & $(0.000)$ \\
High Emotional Stability, & 0.065 & -0.024 & 0.015 \\
Low Conscientiousness Male (Age 16) & $(0.573)$ & $(0.509)$ & $(0.693)$ \\
Low Emotional Stability, & & & \\
High Conscientiousness Male (Age 16) & $0.218^{* *}$ & -0.036 & 0.033 \\
Low Emotional Stability, & $(0.033)$ & $(0.206)$ & $(0.388)$ \\
Low Conscientiousness Male (Age 16) & 0.106 & $-0.060^{*}$ & 0.010 \\
Male & $(0.321)$ & $(0.080)$ & $(0.781)$ \\
& & & \\
$N$ & 0.071 & -0.015 & 0.011 \\
$R^{2}$ & $(0.182)$ & $(0.299)$ & $(0.595)$ \\
\hline
\end{tabular}

Source: British Cohort Study (BCS70), own calculations.

Note: $p$-values in parentheses ${ }^{*} p<0.10,{ }^{* *} p<0.05,{ }^{* * *} p<0.01$. Poor and academic education are binary variables, whereby the values in brackets are coded as 1 . The reference group for the personality types comprises emotionally stable and highly conscientious individuals. The regressions include controls for mother's age, mother's age squared, region of living and parental socioeconomic background at birth, the personality traits agreeableness and extraversion measured at the age of 16 as well as cognitive ability measured at the age of 10 . 
Table 3.17: Personality Change and Educational Qualification

\begin{tabular}{|c|c|c|c|}
\hline Education Level (Age 30) ... & NVQ $(0-5)$ & Poor $(0,1)$ & Academic $(4,5)$ \\
\hline Emotional Stability (Age 10) & $\begin{array}{l}0.092^{* *} \\
(0.015)\end{array}$ & $\begin{array}{l}-0.014 \\
(0.278)\end{array}$ & $\begin{array}{l}0.026^{* *} \\
(0.038)\end{array}$ \\
\hline Conscientiousness (Age 10) & $\begin{array}{l}0.114^{* * *} \\
(0.003)\end{array}$ & $\begin{array}{c}-0.024^{*} \\
(0.080)\end{array}$ & $\begin{array}{l}0.030^{* *} \\
(0.015)\end{array}$ \\
\hline Emotional Stability Male (Age 10) & $\begin{array}{l}-0.138^{* * *} \\
(0.009)\end{array}$ & $\begin{array}{l}0.040^{* *} \\
(0.022)\end{array}$ & $\begin{array}{l}-0.023 \\
(0.194)\end{array}$ \\
\hline Conscientiousness Male (Age 10) & $\begin{array}{l}-0.012 \\
(0.811)\end{array}$ & $\begin{array}{l}0.005 \\
(0.760)\end{array}$ & $\begin{array}{l}-0.004 \\
(0.803)\end{array}$ \\
\hline Emotional Stability (Age 16-10) $>0.5$ & $\begin{array}{l}0.119 \\
(0.103)\end{array}$ & $\begin{array}{l}-0.023 \\
(0.292)\end{array}$ & $\begin{array}{l}0.031 \\
(0.222)\end{array}$ \\
\hline Emotional Stability (Age 16-10) $<-0.5$ & $\begin{array}{l}-0.021 \\
(0.728)\end{array}$ & $\begin{array}{l}-0.002 \\
(0.926)\end{array}$ & $\begin{array}{l}-0.014 \\
(0.505)\end{array}$ \\
\hline Conscientiousness (Age 16-10) $>0.5$ & $\begin{array}{l}0.005 \\
(0.954)\end{array}$ & $\begin{array}{l}0.015 \\
(0.537)\end{array}$ & $\begin{array}{l}-0.010 \\
(0.728)\end{array}$ \\
\hline Conscientiousness (Age 16-10) $<-0.5$ & $\begin{array}{l}-0.312^{* * *} \\
(0.000)\end{array}$ & $\begin{array}{l}0.070^{* * *} \\
(0.001)\end{array}$ & $\begin{array}{l}-0.104^{* * *} \\
(0.000)\end{array}$ \\
\hline Emotional Stability Male (Age 16-10) $>0.5$ & $\begin{array}{l}-0.176^{*} \\
(0.085)\end{array}$ & $\begin{array}{l}0.043 \\
(0.161)\end{array}$ & $\begin{array}{l}-0.034 \\
(0.350)\end{array}$ \\
\hline Emotional Stability Male (Age 16-10) $<-0.5$ & $\begin{array}{l}0.077 \\
(0.408)\end{array}$ & $\begin{array}{l}-0.021 \\
(0.460)\end{array}$ & $\begin{array}{l}0.020 \\
(0.555)\end{array}$ \\
\hline Conscientiousness Male (Age 16-10) $>0.5$ & $\begin{array}{l}0.018 \\
(0.871)\end{array}$ & $\begin{array}{l}-0.035 \\
(0.304)\end{array}$ & $\begin{array}{l}0.011 \\
(0.775)\end{array}$ \\
\hline Conscientiousness Male (Age 16-10) $<-0.5$ & $\begin{array}{l}-0.015 \\
(0.872)\end{array}$ & $\begin{array}{l}-0.015 \\
(0.616)\end{array}$ & $\begin{array}{l}0.011 \\
(0.737)\end{array}$ \\
\hline Male & $\begin{array}{l}0.152^{*} \\
(0.063)\end{array}$ & $\begin{array}{l}-0.040^{*} \\
(0.093)\end{array}$ & $\begin{array}{l}-0.002 \\
(0.955)\end{array}$ \\
\hline $\begin{array}{l}N \\
R^{2}\end{array}$ & $\begin{array}{l}4908 \\
0.255\end{array}$ & $\begin{array}{l}4908 \\
0.136\end{array}$ & $\begin{array}{l}4908 \\
0.186\end{array}$ \\
\hline
\end{tabular}

Source: British Cohort Study (BCS70), own calculations.

Note: $p$-values in parentheses ${ }^{*} p<0.10,{ }^{* *} p<0.05,{ }^{* * *} p<0.01$. Poor and academic education are binary variables, whereby the values in brackets are coded as 1 . The regressions include controls for mother's age, mother's age squared, region of living and parental socioeconomic background at birth, the personality traits agreeableness and extraversion measured at the ages of 10 and 16 as well as cognitive ability measured at the age of 10 . 
CHAPTER 4

PESSIMISTIC EXPECTATIONS AND EDUCATIONAL ACHIEVEMENT 


\subsection{Introduction}

Given the relationship between emotional stability, conscientiousness and education outcomes, it is interesting to closer investigate potential mechanisms behind the observed relationship. This might help to further determine high-risk groups and investigate a potential starting point for effective and efficient (pre-)intervention. Personality psychologists argue that personality traits are less likely to change in the short-run. However, thoughts and expectations are directly malleable by the environment (Roberts (2009), see Chapter 2 Figure 2.1).

Accordingly, the question about the role of pessimistic expectations for the association between emotional stability, conscientiousness and educational achievement arises which is investigated by empirically analyzing the relation (1) between emotional stability and conscientiousness at the ages of 10 and 16 and pessimistic expectations at the age of 16 and (2) between emotional stability, conscientiousness and pessimistic expectations at the age of 16 and educational achievement at the age of 30 .

It is hypothesized that pessimistic expectations predict worse education outcomes, because expected benefits of effort decrease, which might translate into lower effort invested. Less emotionally stable individuals are defined as anxious and worrying so that they are expected to be more likely to have pessimistic expectations. This might be related to disengagement and negative experiences, which is in turn assumed to reinforce pessimistic expectations. Conscientiousness is expected to break the vicious circle of negative expectations and experiences. Conscientious individuals are assumed to be more self-disciplined and more likely to overcome avoidance temperament. Moreover, they are more likely to apply problem-focused strategies such as conducting an appropriate exam preparation which increases control and the probability of making experiences of success and mastering.

Therefore, it is hypothesized that pessimistic expectations partially mediate the relation between emotional stability, conscientiousness and educational achievement. Mediation means that pessimistic expectations are related to emotional stability, conscientiousness and educational achievement, whereby pessimistic expectations account for some of the relation between personality and education outcomes. However, partial mediation means that both personality traits and pessimistic expectations also have an independent direct association with educational achievement. 
Accordingly, also environmental factors such as social and economic conditions are likely to influence beliefs and expectations (Roberts, 2009).

Studies already show that low emotional stability and low conscientiousness are positively related to academic procrastination (Johnson and Bloom, 1995) and that procrastination is negatively related to educational achievement (De Paola and Scoppa, 2015). However, the potential channel of negative expectations is less investigated yet. In the next sections, the data are introduced and the results are discussed. The final section concludes.

\subsection{Data}

The analysis is based on the BCS70, which follows the lives of 17,196 people born in England, Scotland and Wales during one week in 1970. The BCS70 provides detailed information about the individual's family background, cognitive ability, personality and educational achievement. Chapter 3 Appendix Table 3.6 provides an overview of the BCS70 datasets used in the analysis, the variables investigated and the overall number of observations in each of the eight waves.

The analysis relies on a working sample restricted to cases with available information on cognitive ability at the age of 10, personality at the age of 10 and 16, mother's age, socioeconomic background and region of living at birth, expectations about the own ability in exams as well as educational achievement at the age of 30. Only individuals who have no missing values are taken into account. The working sample is presented in Appendix Table 4.4 and consists of 2308 observations.

The initial number of observations presented in Chapter 3 Appendix Table 3.6 is not congruent with the actual number of observations used in the analysis due to a considerable amount of panel attrition and missing values within the variables of interest. The issue of sample selection is addressed in Appendix Table 4.4 that compares the working sample consisting of 2308 observations with the unrestricted sample. Based on these results, it can be concluded that the working sample is positively selected. Therefore, it is likely to estimate lower bound results and that the observed associations are even larger in the unrestricted sample. For a more detailed discussion see Chapter 3 Section 3.2.

To test the robustness of the results, multiple imputation of missing values with 
Figure 4.1: Educational Achievement (NVQ) at Age 30

Female
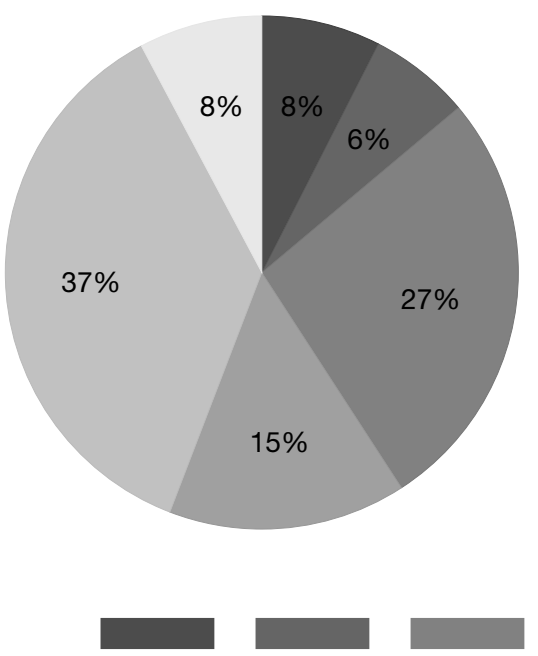

0

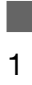

1

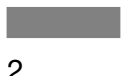

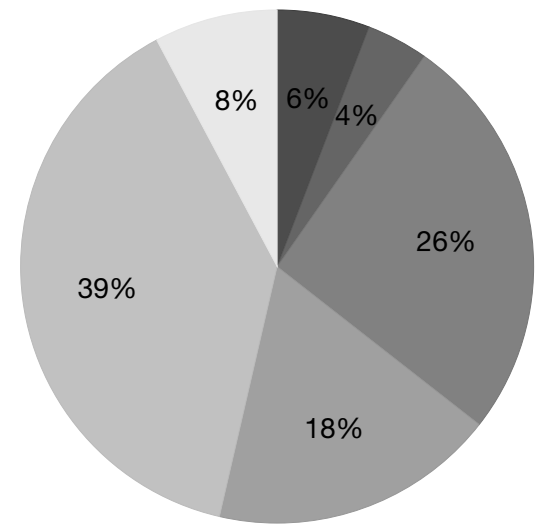

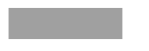

3
Male

5

Source: BCS70. Own calculations, $\mathrm{n}=2308$.

regard to the personality trait variables is performed. Personality items are imputed if there are five or fewer missing values out of 22 items. Imputation of missing values results in an increased sample size to 2614 observations. The results are consistent with the main results and available in Appendix Subsection 4.5.2.2.

\subsubsection{Educational Achievement}

Three dependent variables are used in the analysis. First, the highest National Vocational Qualification (NVQ) at the age of 30 is analyzed which comprises both academic and vocational qualifications (Figure 4.1). NVQ 0 means no qualification level reached, NVQ 1 equals a low Ordinary Level (O level) or a Certificate of Secondary Education (CSE) at grades 2 to 5, NVQ 2 includes a good O level, two or more AS (Advanced Subsidiary) levels or one A (Advanced) level, NVQ 3 equals more than one A level, NVQ 4 refers to a bachelor's or master's degree or PGCE (Postgraduate Certificate in Education) and NVQ 5 means higher degree such as 
doctorate (Dodgeon et al., 2011). Second, a binary variable that specifically accounts for the low educational achievers is created. The variable is 1 if the respondent has only reached NVQ 0 or 1 , while it is 0 if the respondent has reached NVQ 2 or higher at the age of 30. Finally, a binary measure of reaching an academic degree is constructed. The variable is 1 if the respondent has reached NVQ 4 or 5 , while it is 0 if the respondent has NVQ 0,1, 2 or 3 at the age of 30. Figure 4.1 provides information about the distribution of educational achievement at the age of 30 based on 2308 observations.

\subsubsection{Pessimistic Expectations}

A school related indicator of beliefs and expectations about the own ability at the age of 16 is the questions "I am good in exams". The statement "I am good in exams" has the valid answer categories 1 "Applies very much", 2 "Applies somewhat" and 3 "Does not apply". The answer categories "not asked", "not stated" and "no questionnaire" are recoded as missing values. Figure 4.2 provides an overview of the distribution of the variable and shows that females are more likely to have pessimistic expectations with regard to their own ability in exams than males. $22 \%$ of females respond that the statement "does not apply", while only $16 \%$ of males provide the same answer. In contrast, $22 \%$ of males believe that the statement "applies very much", while only $15 \%$ of females have this perception. This finding is in line with the results regarding gender differences in pessimistic expectations in Chapter 6.

\subsubsection{Personality Traits}

In the analysis, personality traits at the ages of 10 and 16 are used threefold: First, they are used as continuous variables as shown in Figure 4.3. Second, four personality types capturing differences in emotional stability and conscientiousness are created. Thereby, the distribution of the standardized variables emotional stability and conscientiousness is split at the mean, i.e. at zero, and four above- and belowmean combinations of emotional stability and conscientiousness are constructed: (1) more emotionally stable and more conscientious, (2) more emotionally stable and less conscientious, (3) less emotionally stable and more conscientious, and (4) less 
Figure 4.2: Exam Expectations at Age 16

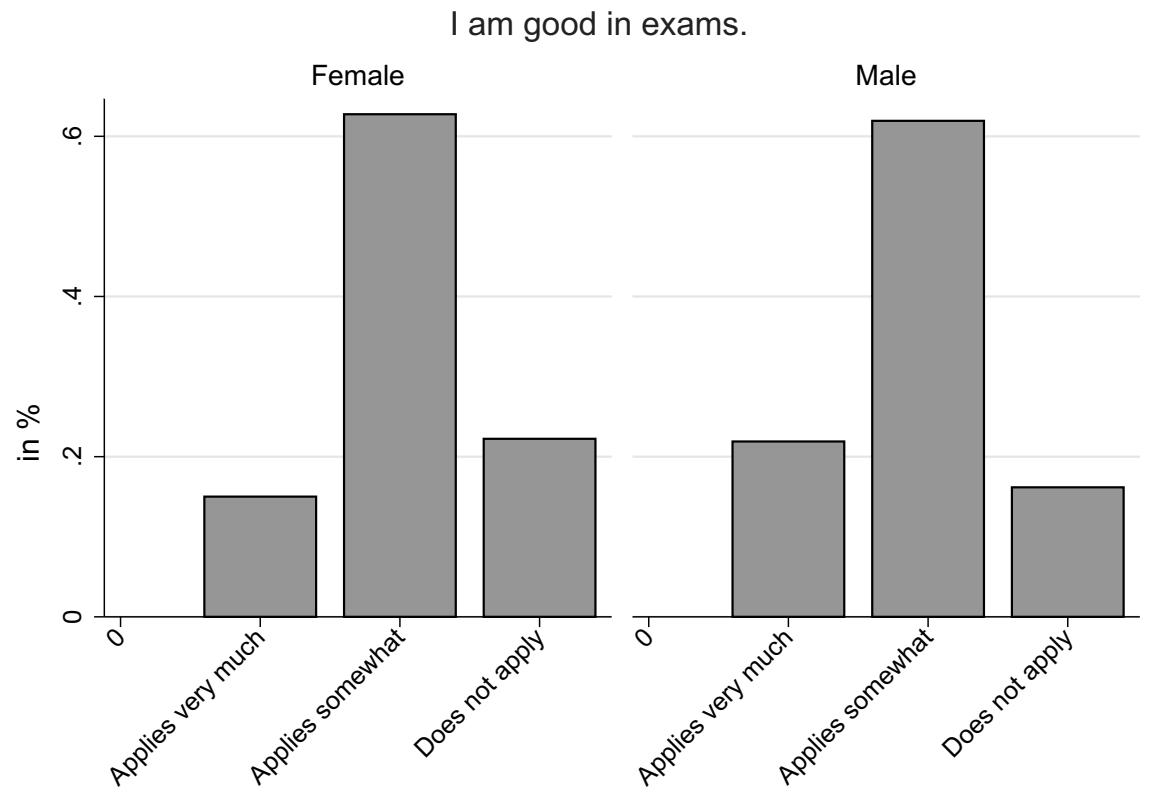

Source: BCS70. Own calculations, $\mathrm{n}=2308$.

emotionally stable and less conscientious individuals. Third, the change in personality between the ages of 10 and 16 is used by subtracting the standardized personality score at the age of 10 from the standardized score at the age of 16 .

A detailed discussion about the personality trait variables used in the analysis is provided in Chapter 3 Subsection 3.2.2. In this chapter, the number of observations varies due to the inclusion of the variable pessimistic expectations. An overview of the reliability measures that correspond to the four personality traits is provided in Appendix Table 4.5. The distributions of emotional stability, conscientiousness, agreeableness and extraversion are presented in Figure 4.3.

Appendix Table 4.6 provides a correlation matrix between emotional stability, conscientiousness, exam expectations and education outcomes. Appendix Table 4.7 shows the share of personality types by gender and related mean education outcomes. And, Appendix Table 4.8 presents the distribution of exam expectations by personality types and gender. It is shown that the most pessimistic expectations 
Figure 4.3: Personality Traits at Ages 10 and 16
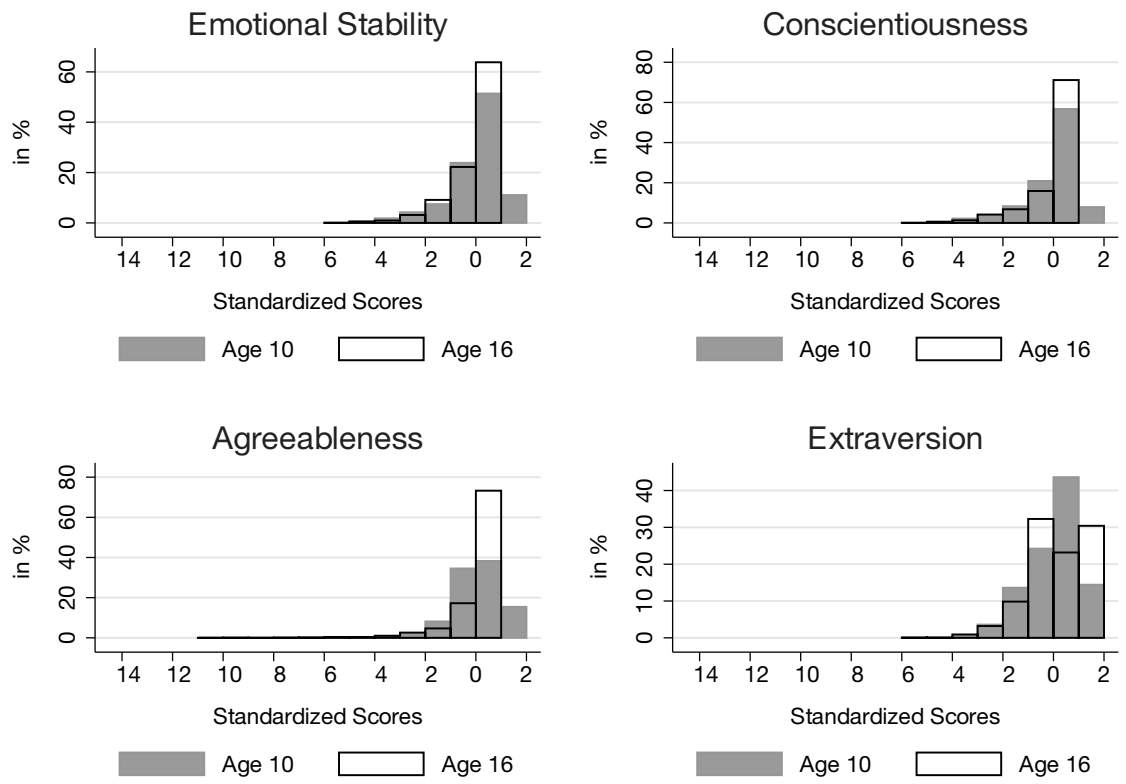

Source: BCS70. Own calculations, $\mathrm{n}=2308$.

have both low emotionally stable and low conscientious females (36\%) and males $(26 \%)$.

\subsubsection{Additional Controls}

In addition to the variables discussed above, controls are included for the individual's cognitive ability at the age of 10 and the individual family background at birth. Detailed information is provided in Chapter 3 Subsection 3.2.3.

\subsection{Results}

The results are based upon OLS and linear probability regression methods. The regressions focus on the relation between emotional stability and conscientiousness, pessimistic expectations and education outcomes at the age of 30 . In all of the re- 
gressions, controls are included for the respondent's cognitive ability, the personality traits agreeableness and extraversion, parental socioeconomic background and region of living at birth as well as the mother's age. To account for the possible problem of heteroscedasticity, robust standard errors are calculated.

\subsubsection{Personality and Pessimistic Expectations}

Table 4.11 addresses the relationship between emotional stability and conscientiousness at the ages of 10 and 16 and pessimistic expectations about one's own ability regarding exams at the age of 16 . The dependent variable "I am good in exams" is an ordinal variable. Therefore, an ordered logit regression model would be appropriate. However, in order to simplify the presentation and discussion of the results, an OLS regression model is provided, while the ordered logit regression model is presented in Appendix Subsection 4.5.2.1. The results based on both regression methods are consistent.

For girls, the analysis indicates that both emotional stability and conscientiousness at the age of 10 are significantly negatively related to the expectation of "I am good in exams: does not apply". An emotional stability increase between the ages of 10 and 16 is significantly related to less pessimistic expectations regarding the own ability in exams, while a decrease in conscientiousness between the ages of 10 and 16 is significantly associated with an increase in pessimistic expectations compared to those girls who do not experience a change in the personality traits emotional stability and conscientiousness between the ages of 10 and 16 .

For boys, emotional stability is less of concern, while only conscientiousness at the age of 10 is significantly related to exam expectations. There are no significant associations between personality change and pessimistic expectations. These results are mainly in line with the findings in Chapter 3. The findings suggest that boys have a significantly better expectations about their own ability in exams compared to girls which is in accordance with the findings in Chapter 6.

Moreover, it is shown that cognitive ability is negatively related to pessimistic exam expectations and likely to be related to a higher probability of making experiences of success and mastering in school. 
Table 4.1: Personality and Pessimistic Expectations

\begin{tabular}{|c|c|}
\hline Pessimistic Expectations (Age 16) ... & I am good in exams (reverse) \\
\hline Emotional Stability (Age 10) & $\begin{array}{l}-0.054^{* *} \\
(0.031)\end{array}$ \\
\hline Conscientiousness (Age 10) & $\begin{array}{l}-0.073^{* * *} \\
(0.003)\end{array}$ \\
\hline Emotional Stability Male (Age 10) & $\begin{array}{l}0.061 \\
(0.104)\end{array}$ \\
\hline Conscientiousness Male (Age 10) & $\begin{array}{l}0.024 \\
(0.483)\end{array}$ \\
\hline Emotional Stability (Age 16-10) $>0.5$ & $\begin{array}{l}-0.111^{* *} \\
(0.019)\end{array}$ \\
\hline Emotional Stability (Age 16-10) $<-0.5$ & $\begin{array}{l}0.032 \\
(0.399)\end{array}$ \\
\hline Conscientiousness (Age 16-10) $>0.5$ & $\begin{array}{l}-0.020 \\
(0.675)\end{array}$ \\
\hline Conscientiousness (Age 16-10) $<-0.5$ & $\begin{array}{l}0.170^{* * *} \\
(0.000)\end{array}$ \\
\hline Emotional Stability Male (Age 16-10) > 0.5 & $\begin{array}{l}0.174^{* *} \\
(0.013)\end{array}$ \\
\hline Emotional Stability Male (Age 16-10) $<-0.5$ & $\begin{array}{l}0.017 \\
(0.808)\end{array}$ \\
\hline Conscientiousness Male (Age 16-10) $>0.5$ & $\begin{array}{l}0.019 \\
(0.801)\end{array}$ \\
\hline Conscientiousness Male (Age 16-10) $<-0.5$ & $\begin{array}{c}-0.110^{*} \\
(0.083)\end{array}$ \\
\hline Male & $\begin{array}{l}-0.131^{* *} \\
(0.021)\end{array}$ \\
\hline Cognitive Ability (Age 10) & $\begin{array}{l}-0.203^{* * *} \\
(0.000)\end{array}$ \\
\hline Higher SES Parents (Birth) & $\begin{array}{l}-0.029 \\
(0.337)\end{array}$ \\
\hline $\begin{array}{l}N \\
R^{2}\end{array}$ & $\begin{array}{l}2308 \\
0.173\end{array}$ \\
\hline
\end{tabular}

Source: British Cohort Study (BCS70), own calculations.

Note: $p$-values in parentheses ${ }^{*} p<0.10,{ }^{* *} p<0.05,{ }^{* * *} p<0.01$. The OLS regressions include controls for mother's age, mother's age squared, region of living at birth as well as the personality traits agreeableness and extraversion measured at the ages of 10 and 16 . 


\subsubsection{Predictive Power of Pessimistic Expectations}

Table 4.2 targets the predictive power of pessimistic expectations regarding one's own ability in exams at the age of 16 for educational achievement at the age of 30 . The results show that women who respond that the statement "I am good in exams: applies somewhat" reach a 0.53 lower NVQ level, have an almost 4 percentage points higher probability of having no or limited education outcomes and a 21 percentage points lower probability of holding an academic degree at the age of 30 compared to women who state "I am good in exams: applies very much". Men who respond "I am good in exams: applies somewhat" have significantly improved education outcomes compared to women. This result is in line with Chapter 6 and suggests that women are particularly vulnerable to pessimistic expectations.

Table 4.2: Role of Pessimistic Expectations

\begin{tabular}{|c|c|c|c|c|c|c|}
\hline \multirow{2}{*}{$\begin{array}{l}\text { Education Level (Age 30) ... } \\
\text { High Emotional Stability, } \\
\text { Low Conscientiousness } \\
\text { (Age 16) }\end{array}$} & \multicolumn{2}{|l|}{ NVQ (0-5) } & \multicolumn{2}{|c|}{ Poor $(0,1)$} & \multicolumn{2}{|c|}{ Academic $(4,5)$} \\
\hline & $\begin{array}{l}-0.427^{* * *} \\
(0.000)\end{array}$ & $\begin{array}{l}-0.335^{* * *} \\
(0.005)\end{array}$ & $\begin{array}{l}0.058 \\
(0.108)\end{array}$ & $\begin{array}{l}0.049 \\
(0.180)\end{array}$ & $\begin{array}{l}-0.141^{* * *} \\
(0.002)\end{array}$ & $\begin{array}{l}-0.106^{* *} \\
(0.017)\end{array}$ \\
\hline $\begin{array}{l}\text { Low Emotional Stability, } \\
\text { High Conscientiousness } \\
\text { (Age 16) }\end{array}$ & $\begin{array}{l}-0.227^{* * *} \\
(0.009)\end{array}$ & $\begin{array}{l}-0.194^{* *} \\
(0.022)\end{array}$ & $\begin{array}{l}0.047^{* *} \\
(0.046)\end{array}$ & $\begin{array}{l}0.043^{*} \\
(0.062)\end{array}$ & $\begin{array}{l}-0.049 \\
(0.128)\end{array}$ & $\begin{array}{l}-0.036 \\
(0.243)\end{array}$ \\
\hline $\begin{array}{l}\text { Low Emotional Stability, } \\
\text { Low Conscientiousness } \\
\text { (Age 16) }\end{array}$ & $\begin{array}{l}-0.480^{* * *} \\
(0.000)\end{array}$ & $\begin{array}{l}-0.390 * * * \\
(0.000)\end{array}$ & $\begin{array}{l}0.088^{* * * *} \\
(0.005)\end{array}$ & $\begin{array}{l}0.079 * * \\
(0.013)\end{array}$ & $\begin{array}{l}-0.137^{* * *} \\
(0.000)\end{array}$ & $\begin{array}{l}-0.103^{* * *} \\
(0.006)\end{array}$ \\
\hline $\begin{array}{l}\text { High Emotional Stability, } \\
\text { Low Conscientiousness } \\
\text { Male (Age 16) }\end{array}$ & $\begin{array}{l}0.145 \\
(0.387)\end{array}$ & $\begin{array}{l}0.051 \\
(0.760)\end{array}$ & $\begin{array}{l}-0.051 \\
(0.279)\end{array}$ & $\begin{array}{l}-0.039 \\
(0.410)\end{array}$ & $\begin{array}{l}0.024 \\
(0.707)\end{array}$ & $\begin{array}{l}-0.006 \\
(0.923)\end{array}$ \\
\hline $\begin{array}{l}\text { Low Emotional Stability, } \\
\text { High Conscientiousness } \\
\text { Male (Age 16) }\end{array}$ & $\begin{array}{l}0.318^{* *} \\
(0.023)\end{array}$ & $\begin{array}{l}0.292^{* *} \\
(0.034)\end{array}$ & $\begin{array}{l}-0.062^{*} \\
(0.077)\end{array}$ & $\begin{array}{l}-0.060^{*} \\
(0.087)\end{array}$ & $\begin{array}{l}0.028 \\
(0.630)\end{array}$ & $\begin{array}{l}0.017 \\
(0.758)\end{array}$ \\
\hline $\begin{array}{l}\text { Low Emotional Stability, } \\
\text { Low Conscientiousness } \\
\text { Male (Age 16) }\end{array}$ & $\begin{array}{l}-0.010 \\
(0.948)\end{array}$ & $\begin{array}{l}-0.073 \\
(0.646)\end{array}$ & $\begin{array}{l}-0.042 \\
(0.368)\end{array}$ & $\begin{array}{l}-0.038 \\
(0.424)\end{array}$ & $\begin{array}{l}-0.056 \\
(0.348)\end{array}$ & $\begin{array}{l}-0.079 \\
(0.190)\end{array}$ \\
\hline $\begin{array}{l}\text { Good in Exams: } \\
\text { Applies somewhat } \\
\text { (Age 16) }\end{array}$ & & $\begin{array}{l}-0.529 * * * \\
(0.000)\end{array}$ & & $\begin{array}{l}0.039 * * \\
(0.011)\end{array}$ & & $\begin{array}{l}-0.209^{* * *} \\
(0.000)\end{array}$ \\
\hline $\begin{array}{l}\text { Good in Exams: } \\
\text { Does not apply }\end{array}$ & & $\begin{array}{l}-0.831^{* * *} \\
(0.000)\end{array}$ & & $\begin{array}{l}0.083^{* * *} \\
(0.001)\end{array}$ & & $\begin{array}{l}-0.316^{* * *} \\
(0.000)\end{array}$ \\
\hline
\end{tabular}


... Table 4.2 continued

\begin{tabular}{|c|c|c|c|c|c|c|}
\hline Education Level (Age 30) ... & \multicolumn{2}{|c|}{ NVQ $(0-5)$} & \multicolumn{2}{|l|}{ Poor $(0,1)$} & \multicolumn{2}{|c|}{ Academic $(4,5)$} \\
\hline $\begin{array}{l}\text { Good in Exams: } \\
\text { Applies somewhat } \\
\text { (Male, Age 16) }\end{array}$ & & $\begin{array}{l}0.389^{* * *} \\
(0.001)\end{array}$ & & $\begin{array}{l}-0.049^{* *} \\
(0.035)\end{array}$ & & $\begin{array}{l}0.111^{* *} \\
(0.024)\end{array}$ \\
\hline $\begin{array}{l}\text { Good in Exams: } \\
\text { Does not apply } \\
\text { (Male, Age } 16 \text { ) }\end{array}$ & & $\begin{array}{l}0.157 \\
(0.331)\end{array}$ & & $\begin{array}{l}0.022 \\
(0.625)\end{array}$ & & $\begin{array}{l}0.075 \\
(0.220)\end{array}$ \\
\hline Male & $\begin{array}{l}0.098 \\
(0.168)\end{array}$ & $\begin{array}{l}-0.199 * * \\
(0.049)\end{array}$ & $\begin{array}{l}-0.022 \\
(0.198)\end{array}$ & $\begin{array}{l}0.008 \\
(0.702)\end{array}$ & $\begin{array}{l}0.033 \\
(0.254)\end{array}$ & $\begin{array}{l}-0.061 \\
(0.171)\end{array}$ \\
\hline $\begin{array}{l}\text { Cognitive Ability } \\
\text { (Age 10) }\end{array}$ & $\begin{array}{l}0.462^{* * *} \\
(0.000)\end{array}$ & $\begin{array}{l}0.389 * * * \\
(0.000)\end{array}$ & $\begin{array}{l}-0.083^{* * *} \\
(0.000)\end{array}$ & $\begin{array}{l}-0.075^{* * *} \\
(0.000)\end{array}$ & $\begin{array}{l}0.140^{* * *} \\
(0.000)\end{array}$ & $\begin{array}{l}0.112^{* * *} \\
(0.000)\end{array}$ \\
\hline $\begin{array}{l}\text { Higher SES Parents } \\
\text { (Birth) }\end{array}$ & $\begin{array}{l}0.343^{* * *} \\
(0.000)\end{array}$ & $\begin{array}{l}0.329^{* * *} \\
(0.000)\end{array}$ & $\begin{array}{l}-0.033^{* *} \\
(0.012)\end{array}$ & $\begin{array}{l}-0.032^{* *} \\
(0.015)\end{array}$ & $\begin{array}{l}0.147^{* * *} \\
(0.000)\end{array}$ & $\begin{array}{l}0.142^{* * *} \\
(0.000)\end{array}$ \\
\hline $\begin{array}{l}N \\
R^{2}\end{array}$ & $\begin{array}{l}2308 \\
0.247\end{array}$ & $\begin{array}{l}2308 \\
0.274\end{array}$ & $\begin{array}{l}2308 \\
0.111\end{array}$ & $\begin{array}{l}2308 \\
0.120\end{array}$ & $\begin{array}{l}2308 \\
0.190\end{array}$ & $\begin{array}{l}2308 \\
0.216\end{array}$ \\
\hline
\end{tabular}

Source: British Cohort Study (BCS70), own calculations.

Note: $p$-values in parentheses $* p<0.10,{ }^{* *} p<0.05,{ }^{* * *} p<0.01$. Poor and academic education are binary variables, whereby the values in brackets are coded as 1 . The reference group for the personality types comprises more emotionally stable and more conscientious individuals. The reference group for the exam expectations are individuals who respond that the statement "I am good in exams" applies very much. The OLS regressions include controls for mother's age, mother's age squared, region of living at birth as well as the personality traits agreeableness and extraversion measured at the age of 16 .

For the statement "I am good in exams: does not apply", there are no gender differences. Individuals who believe "I am good in exams: does not apply" reach a 0.83 lower NVQ level, have an 8 percentage points higher probability of having no or limited educational achievement and have a 32 percentage points lower probability of holding an academic degree at the age of 30 compared to pupils who believe that the statement "I am good in exams: applies very much".

Furthermore, the results in Table 4.2 reveal that after including the pessimistic expectation dummies into the regression, the coefficient size of the personality types decrease. For instance, scoring below average in emotional stability and conscientiousness is related to a 9 percentage points higher probability of having no or limited education outcomes at the age of 30 . After introducing the pessimistic expectation 
dummies into the regression, the coefficient for individuals who score below average in emotional stability and conscientiousness is decreasing from 9 to 8 percentage points higher probability of having no or limited educational achievement at the age of 30 compared to the reference group. These decreases are not significant, but consistent for all personality types.

This indicates that pessimistic expectations partially mediate the relation between personality and educational achievement. Mediation means that pessimistic expectations are related to emotional stability, conscientiousness and educational achievement, whereby pessimistic expectations account for some of the relation between personality and education outcomes. However, partial mediation means that both personality traits and pessimistic expectations also have an independent direct association with educational achievement. Accordingly, various findings emphasize that also environmental factors such as family, teacher or peer effects are likely to influence beliefs and expectations. The results are in line with these theoretical considerations.

Moreover, it is interesting to know whether the predictive power of pessimistic expectations for educational attainment varies with personality characteristics. To test this, a dummy for having severe pessimistic expectations is built. It has the value 1 if the individual responds that the statement "I am good in exams: does not apply". The dummy is interacted with the personality types comprising (1) more emotionally stable and more conscientious, (2) more emotionally stable and less conscientious, (3) less emotionally stable and more conscientious and (4) less emotionally stable and less conscientious individuals. The reference group comprises low emotionally stable and low conscientious individuals who do have pessimistic expectations, i.e. theoretically considered as the group with the highest risk for educational failure.

Table 4.3 reveals that less pessimistic individuals who are both low emotionally stable and low conscientious reach a 0.28 higher NVQ level and an 8.8 percentage points higher probability of holding an academic degree at the age of 30 compared to more pessimistic individuals with similar personality characteristics. The analysis shows that also the other personality types have significantly better education outcomes if they do not have pessimistic expectations regarding their own ability in exams. The negative effect of pessimistic expectations seems to decrease with potentially less problematic personality characteristics. This result is in line with the theoretical considerations. It suggests that pessimistic expectations are predictive 
for educational achievement and that targeting pessimistic expectations might help to boost education outcomes.

Finally, the results suggest that low emotionally stable, but more conscientious men who have pessimistic expectations have significantly better education outcomes compared to women with the same characteristics. In addition, the results indicate that these men even overcompensate in comparison to more emotionally stable and more conscientious ones who have no pessimistic expectations.

Table 4.3: Interaction between Personality and Pessimistic Expectations

\begin{tabular}{|c|c|c|c|}
\hline Education Level (Age 30) ... & NVQ (0-5) & Poor $(0,1)$ & Academic $(4,5)$ \\
\hline $\begin{array}{l}\text { High Emotional Stability, High Conscientiousness } \\
\text { Good in Exams: Applies very much, somewhat } \\
\text { (Age 16) }\end{array}$ & $\begin{array}{l}0.725^{* * *} \\
(0.000)\end{array}$ & $\begin{array}{l}-0.117^{* *} \\
(0.022)\end{array}$ & $\begin{array}{l}0.217^{* * *} \\
(0.000)\end{array}$ \\
\hline $\begin{array}{l}\text { High Emotional Stability, High Conscientiousness } \\
\text { Good in Exams: Does not apply (Age 16) }\end{array}$ & $\begin{array}{l}0.447^{* *} \\
(0.011)\end{array}$ & $\begin{array}{l}-0.096^{*} \\
(0.096)\end{array}$ & $\begin{array}{l}0.110^{*} \\
(0.069)\end{array}$ \\
\hline $\begin{array}{l}\text { High Emotional Stability, Low Conscientiousness } \\
\text { Good in Exams: Applies very much, somewhat } \\
\text { (Age 16) }\end{array}$ & $\begin{array}{l}0.406^{* *} \\
(0.037)\end{array}$ & $\begin{array}{l}-0.051 \\
(0.417)\end{array}$ & $\begin{array}{l}0.126^{*} \\
(0.060)\end{array}$ \\
\hline $\begin{array}{l}\text { High Emotional Stability, Low Conscientiousness } \\
\text { Good in Exams: Does not apply (Age 16) }\end{array}$ & $\begin{array}{l}-0.116 \\
(0.601)\end{array}$ & $\begin{array}{l}-0.057 \\
(0.475)\end{array}$ & $\begin{array}{l}-0.099 \\
(0.150)\end{array}$ \\
\hline $\begin{array}{l}\text { Low Emotional Stability, High Conscientiousness } \\
\text { Good in Exams: Applies very much, somewhat } \\
\text { (Age 16) }\end{array}$ & $\begin{array}{l}0.577^{* * * *} \\
(0.000)\end{array}$ & $\begin{array}{c}-0.099 * \\
(0.060)\end{array}$ & $\begin{array}{l}0.192^{* * *} \\
(0.000)\end{array}$ \\
\hline $\begin{array}{l}\text { Low Emotional Stability, High Conscientiousness } \\
\text { Good in Exams: Does not apply (Age 16) }\end{array}$ & $\begin{array}{l}-0.027 \\
(0.895)\end{array}$ & $\begin{array}{l}0.057 \\
(0.438)\end{array}$ & $\begin{array}{l}-0.008 \\
(0.892)\end{array}$ \\
\hline $\begin{array}{l}\text { Low Emotional Stability, Low Conscientiousness } \\
\text { Good in Exams: Applies very much, somewhat } \\
\text { (Age 16) }\end{array}$ & $\begin{array}{l}0.280^{*} \\
(0.086)\end{array}$ & $\begin{array}{l}-0.034 \\
(0.544)\end{array}$ & $\begin{array}{l}0.088^{*} \\
(0.094)\end{array}$ \\
\hline $\begin{array}{l}\text { High Emotional Stability, High Conscientiousness } \\
\text { Good in Exams: Applies very much, somewhat } \\
\text { (Male, Age 16) }\end{array}$ & $\begin{array}{l}0.363 \\
(0.208)\end{array}$ & $\begin{array}{l}-0.126 \\
(0.175)\end{array}$ & $\begin{array}{l}0.090 \\
(0.296)\end{array}$ \\
\hline $\begin{array}{l}\text { High Emotional Stability, High Conscientiousness } \\
\text { Good in Exams: Does not apply (Male, Age 16) }\end{array}$ & $\begin{array}{l}-0.060 \\
(0.859)\end{array}$ & $\begin{array}{l}-0.014 \\
(0.903)\end{array}$ & $\begin{array}{l}-0.035 \\
(0.741)\end{array}$ \\
\hline $\begin{array}{l}\text { High Emotional Stability, Low Conscientiousness } \\
\text { Good in Exams: Applies very much, somewhat }\end{array}$ & $\begin{array}{l}0.308 \\
(0.338)\end{array}$ & $\begin{array}{l}-0.161 \\
(0.116) \\
\text { Contint }\end{array}$ & $\begin{array}{l}0.032 \\
(0.752) \\
\text { on next page. }\end{array}$ \\
\hline
\end{tabular}


... Table 4.3 continued

\begin{tabular}{llll}
\hline Education Level (Age 30) $\ldots$ & NVQ $(0-5)$ & Poor $(0,1)$ & Academic $(4,5)$ \\
\hline (Male, Age 16) & & & \\
High Emotional Stability, Low Conscientiousness & 0.688 & -0.159 & $0.226^{*}$ \\
Good in Exams: Does not apply (Male, Age 16) & $(0.114)$ & $(0.233)$ & $(0.093)$ \\
& & & \\
Low Emotional Stability, High Conscientiousness & $0.564^{*}$ & -0.135 & 0.089 \\
Good in Exams: Applies very much, somewhat & $(0.052)$ & $(0.149)$ & $(0.325)$ \\
(Male, Age 16) & & & \\
Low Emotional Stability, High Conscientiousness & $0.781^{* *}$ & $-0.308^{* *}$ & 0.127 \\
Good in Exams: Does not apply (Male, Age 16) & $(0.049)$ & $(0.012)$ & $(0.323)$ \\
& & & \\
Low Emotional Stability, Low Conscientiousness & 0.342 & $-0.192^{* *}$ & 0.012 \\
Good in Exams: Applies very much, somewhat & $(0.240)$ & $(0.045)$ & $(0.892)$ \\
(Male, Age 16) & & & \\
Male & -0.214 & 0.088 & -0.044 \\
\hline$N$ & $(0.428)$ & $(0.328)$ & $(0.566)$ \\
\hline$R^{2}$ & 2308 & 2308 & 2308 \\
\hline
\end{tabular}

Source: British Cohort Study (BCS70), own calculations.

Note: $p$-values in parentheses $* p<0.10,{ }^{* *} p<0.05, * * * p<0.01$. Poor and academic education are binary variables, whereby the values in brackets are coded as 1 . The reference group for the personality types comprises low emotionally stable and low conscientious individuals who have pessimistic exam expectations. The OLS regressions include controls for mother's age, mother's age squared, region of living and parental socioeconomic background at birth, the personality traits agreeableness and extraversion measured at the age of 16 as well as cognitive ability measured at the age of 10 .

\subsection{Conclusion}

Given the relationship between emotional stability, conscientiousness and educational achievement, the question about the role of pessimistic expectation arises. This question is investigated by analyzing the relation (1) between emotional stability, conscientiousness and pessimistic expectations at the ages of 10 and 16 and (2) between emotional stability, conscientiousness and pessimistic expectations at the age of 16 and educational attainment at the age of 30 .

The main results are summarized as follows: First, the analysis shows that pessimistic expectations regarding one's own ability in exams at the age of 16 are quite 
common among teenagers. 20\% of teenagers have the perception that the general statement "I am good in exams" does not apply. The analysis shows that girls are more pessimistic than boys. $22 \%$ of girls respond that the statement "I am good in exams" does not apply, while only $16 \%$ of boys provide the same answer.

For women, emotional stability and conscientiousness at the age of 10 are significantly associated with pessimistic expectations about the own ability in exams even after controlling for cognitive ability. Moreover, an increase in emotional stability between the ages of 10 and 16 is associated with less pessimistic expectations, while a decrease in conscientiousness is related to worse perceptions about the own ability in exams. This is in line with the theoretical considerations. In contrast, only conscientiousness at the age of 10 is significantly associated with the pessimistic expectations for men.

Second, the results suggest that pessimistic expectations at the age of 16 significantly predict educational achievement at the age of 30 after controlling for personality traits and cognitive ability and that those pessimistic expectations partially mediate the relation between personality and educational achievement. However, the negative association between pessimistic expectations and educational achievement is stronger for women compared to men. This suggests that women are more likely to have pessimistic expectations and that they are more vulnerable to pessimistic expectations compared to men. This finding is in line with the results regarding gender differences in pessimistic expectations presented in Chapter 6.

The results show that low emotionally stable, but more conscientious men who have pessimistic expectations have significantly improved education outcomes compared to women with similar characteristics. Arguing in terms of the model it is likely that these men act in contrast to the predictions of the model. They might force themselves to overcome pessimistic expectations more intensively by putting effort instead of avoiding challenging situations. This is likely to result in improved education outcomes. These gender differences in coping strategies might be related to variation in societal expectations regarding women and men. Accordingly, Osborne (2000) studies personality differences and labor market outcomes and finds that men face a much higher wage penalty for withdrawal compared to women.

Finally, Chapter 3 Table 3.2 suggests that men have significantly better education outcomes at the age of 30 compared to women. After controlling for personality types (Chapter 3 Table 3.4) size and significance of the male coefficients vanish. In this 
Chapter, the male coefficients even turns negative after controlling for pessimistic expectations (Table 4.2). On the one hand, this means that more emotionally stable and more conscientious men are likely to have worse education outcomes at the age of 30 compared to women with similar personality characteristics. On the other hand, the findings suggest that gender differences regarding the selection in and the reaction to pessimistic expectations might be able to contribute to the explanation of gender differences in educational achievement. This might be particularly relevant in male dominated disciplines such as math or technological studies. Gevrek et al. (2018) find that the gender test score gap in math is smaller in more genderequal countries. Therefore, a reduction of societal gender inequalities might help to decrease downward biased expectations of women and boost their educational achievement in these disciplines.

Are the observed relationships between emotional stability, conscientiousness and pessimistic expectations as well as gender differences solely related to educational achievement or do they also hold regarding more subjective outcomes such as mental health? This question is further investigated in the next chapters. 


\subsection{Appendix}

\subsubsection{Data Description}

Table 4.4: Descriptive Statistics

\begin{tabular}{|c|c|c|c|c|c|c|c|c|c|c|}
\hline \multirow[b]{2}{*}{ Variable Names } & \multicolumn{5}{|c|}{ Working Sample } & \multicolumn{5}{|c|}{ Unrestricted Sample } \\
\hline & $\mathrm{N}$ & Mean & SD & Min & $\operatorname{Max}$ & $\mathrm{N}$ & Mean & SD & Min & $\operatorname{Max}$ \\
\hline Highest NVQ Level (Age 30) & 2308 & 2.95 & 1.33 & 0.00 & 5.00 & 7704 & 2.56 & 1.42 & 0.00 & 5.00 \\
\hline No Qualifications (Age 30) & 2308 & 0.12 & 0.33 & 0.00 & 1.00 & 7704 & 0.20 & 0.40 & 0.00 & 1.00 \\
\hline Academic Degree (Age 30) & 2308 & 0.45 & 0.50 & 0.00 & 1.00 & 7704 & 0.34 & 0.48 & 0.00 & 1.00 \\
\hline Exam: Applies very much (Age 16) & 2308 & 0.18 & 0.38 & 0.00 & 1.00 & 4814 & 0.16 & 0.37 & 0.00 & 1.00 \\
\hline Exam: Applies somewhat (Age 16) & 2308 & 0.62 & 0.48 & 0.00 & 1.00 & 4814 & 0.62 & 0.48 & 0.00 & 1.00 \\
\hline Exam: Does not apply (Age 16) & 2308 & 0.20 & 0.40 & 0.00 & 1.00 & 4814 & 0.22 & 0.41 & 0.00 & 1.00 \\
\hline Emotional Stability (ES, Age 10) & 2308 & 0.12 & 0.92 & -3.64 & 1.33 & 8910 & 0.00 & 1.00 & -4.28 & 1.33 \\
\hline Conscientiousness (C, Age 10) & 2308 & 0.17 & 0.89 & -3.41 & 1.20 & 8910 & 0.00 & 1.00 & -3.45 & 1.20 \\
\hline Agreeableness (A, Age 10) & 2308 & 0.16 & 0.83 & -5.55 & 1.34 & 8910 & 0.00 & 1.00 & -6.64 & 1.34 \\
\hline Extraversion (E, Age 10) & 2308 & 0.04 & 0.97 & -3.76 & 1.61 & 8910 & -0.00 & 1.00 & -4.37 & 1.61 \\
\hline Emotional Stability (ES, Age 16) & 2308 & 0.10 & 0.90 & -4.60 & 0.85 & 6768 & 0.00 & 1.00 & -4.60 & 0.85 \\
\hline Conscientiousness (C, Age 16) & 2308 & 0.16 & 0.86 & -4.76 & 0.71 & 6768 & -0.00 & 1.00 & -4.76 & 0.71 \\
\hline Agreeableness (A, Age 16) & 2308 & 0.16 & 0.79 & -7.55 & 0.52 & 6768 & 0.00 & 1.00 & -8.29 & 0.52 \\
\hline Extraversion (E, Age 16) & 2308 & 0.01 & 0.97 & -5.49 & 0.98 & 6768 & 0.00 & 1.00 & -5.49 & 0.98 \\
\hline High ES, High C (Age 16) & 2308 & 0.53 & 0.50 & 0.00 & 1.00 & 6768 & 0.48 & 0.50 & 0.00 & 1.00 \\
\hline High ES, Low C (Age 16) & 2308 & 0.11 & 0.31 & 0.00 & 1.00 & 6768 & 0.13 & 0.34 & 0.00 & 1.00 \\
\hline Low ES, High C (Age 16) & 2308 & 0.19 & 0.39 & 0.00 & 1.00 & 6768 & 0.17 & 0.38 & 0.00 & 1.00 \\
\hline Low ES, Low C (Age 16) & 2308 & 0.17 & 0.38 & 0.00 & 1.00 & 6768 & 0.22 & 0.41 & 0.00 & 1.00 \\
\hline Male & 2308 & 0.39 & 0.49 & 0.00 & 1.00 & 10046 & 0.50 & 0.50 & 0.00 & 1.00 \\
\hline Cognitive Ability (Age 10) & 2308 & 0.32 & 0.87 & -3.37 & 2.18 & 8586 & -0.00 & 1.00 & -4.10 & 2.18 \\
\hline Higher SES Parents (Birth) & 2308 & 0.24 & 0.42 & 0.00 & 1.00 & 10033 & 0.19 & 0.39 & 0.00 & 1.00 \\
\hline Region (Birth) & 2308 & 5.74 & 2.68 & 1.00 & 10.00 & 10046 & 5.69 & 2.64 & 1.00 & 11.00 \\
\hline Age Mother & 2308 & 23.12 & 3.97 & 14.00 & 42.00 & 9989 & 22.33 & 3.96 & 13.00 & 47.00 \\
\hline
\end{tabular}

Source: BCS70, own calculations. SES refers to the term socioeconomic status. 
Table 4.5: Personality Traits at the Ages of 10 and 16

\begin{tabular}{|c|c|c|c|c|}
\hline $\begin{array}{l}\text { Personality } \\
\text { Trait }\end{array}$ & $\begin{array}{l}\text { Correlation } \\
80-86\end{array}$ & $\begin{array}{l}\text { Explained } \\
\text { Variance }\end{array}$ & $\begin{array}{l}\text { Cronbach's } \\
\text { Alpha }\end{array}$ & Items \\
\hline \multicolumn{5}{|c|}{ Emotional Stability } \\
\hline Age 10 & & $53.16 \%$ & 0.82 & R8. Irritable. \\
\hline Age 16 & 0.39 & $54.75 \%$ & 0.83 & $\begin{array}{l}\text { R9. Miserable and tearful. } \\
\text { C8. Requests must be met. } \\
\text { C12. Sullen or sulky. } \\
\text { C16. Changes mood quickly. } \\
\text { C17. Temper unpredictable. }\end{array}$ \\
\hline \multicolumn{5}{|c|}{ Conscientiousness } \\
\hline Age 10 & & $66.09 \%$ & 0.83 & R15. Cannot settle. \\
\hline Age 16 & 0.42 & $63.18 \%$ & 0.81 & $\begin{array}{l}\text { C3. Easily distracted. } \\
\text { C13. Fails to finish things. } \\
\text { C19. Difficulty concentrating. }\end{array}$ \\
\hline \multicolumn{5}{|c|}{ Agreeableness } \\
\hline Age 10 & & $46.86 \%$ & 0.81 & R3. Destroys belongings. \\
\hline Age 16 & 0.33 & $40.98 \%$ & 0.76 & $\begin{array}{l}\text { R4. Frequently fights. } \\
\text { R10. Takes others' belongings. } \\
\text { R14. Often disobedient. } \\
\text { R18. Often tells lies. } \\
\text { R19. Bullies other children. } \\
\text { C11. Interferes with others. }\end{array}$ \\
\hline \multicolumn{5}{|l|}{ Extraversion } \\
\hline Age 10 & & $38.19 \%$ & 0.57 & R5. Not much liked by others. \\
\hline Age 16 & 0.36 & $36.35 \%$ & 0.55 & $\begin{array}{l}\text { R6. Often worried. } \\
\text { R7. Rather solitary. } \\
\text { R16. Afraid of new things. } \\
\text { R17. Fussy or over-particular. }\end{array}$ \\
\hline
\end{tabular}

Source: BCS70, own calculations based on working sample $\mathrm{n}=2308$.

Note: Items are stated in original terms, but reversely used in the analysis. 
Table 4.6: Personality, Pessimistic Exam Expectations and Educational Achievement

\begin{tabular}{lcccccccc}
\hline \multicolumn{2}{c}{$\begin{array}{c}\text { Female } \\
\text { NVQ }\end{array}$} & Poor & \multicolumn{2}{c}{ AcademicExam } & $\begin{array}{c}\text { Male } \\
\text { NVQ }\end{array}$ & Poor & AcademicExam \\
\hline Emotional Stability & & & & & & & & \\
Age 10 & .14 & -.11 & .12 & -.09 & .10 & - & .14 & -.09 \\
Age 16 & .18 & -.13 & .14 & -.16 & .12 & - & .14 & -.14 \\
& & & & & & & & \\
Conscientiousness & & & & & & & & \\
Age 10 & .21 & -.17 & .15 & -.17 & .23 & -.12 & .20 & -.20 \\
Age 16 & .20 & -.11 & .18 & -.24 & .27 & -.14 & .25 & -.19 \\
Exam & & & & & & & & \\
Age 16 & -.33 & .18 & -.31 & - & -.33 & .21 & -.29 & - \\
\hline
\end{tabular}

Source: BCS70, own calculations based on working sample $\mathrm{n}=2308$.

Note: Correlation coefficients reported are significant at the $5 \%$ level or higher. NVQ $=$ National Vocational Qualification (Scale 0-5), Poor Education Qualification (Binary: 1=0,1), Academic Qualification (Binary: 1=4,5) at the age of 30. Exam expectations (good in exams: $1=$ applies very much, $2=$ applies somewhat, $3=$ does not apply).

Table 4.7: Personality Types and Mean Educational Qualification

\begin{tabular}{|c|c|c|c|c|c|c|}
\hline Personality Type & $\begin{array}{l}\text { All } \\
\text { Share }\end{array}$ & $\varnothing \mathrm{NVQ}$ & $\begin{array}{l}\text { Female } \\
\text { Share }\end{array}$ & $\varnothing \mathrm{NVQ}$ & $\begin{array}{l}\text { Male } \\
\text { Share }\end{array}$ & $\varnothing \mathrm{NVQ}$ \\
\hline $\begin{array}{l}\text { High Emotional Stability, } \\
\text { High Conscientiousness }\end{array}$ & $52 \%$ & 3.19 & $52 \%$ & 3.14 & $53 \%$ & 3.25 \\
\hline $\begin{array}{l}\text { High Emotional Stability, } \\
\text { Low Conscientiousness }\end{array}$ & $11 \%$ & 2.61 & $9 \%$ & 2.52 & $15 \%$ & 2.69 \\
\hline $\begin{array}{l}\text { Low Emotional Stability, } \\
\text { High Conscientiousness }\end{array}$ & $19 \%$ & 2.99 & $21 \%$ & 2.86 & $15 \%$ & 3.28 \\
\hline $\begin{array}{l}\text { Low Emotional Stability, } \\
\text { Low Conscientiousness }\end{array}$ & $18 \%$ & 2.41 & $18 \%$ & 2.39 & $17 \%$ & 2.43 \\
\hline Total & $100 \%$ & 2.95 & $100 \%$ & 2.89 & $100 \%$ & 3.03 \\
\hline
\end{tabular}

Source: BCS70, own calculations based on working sample $\mathrm{n}=2308$.

Note: Column percentages by gender are shown. NVQ $=$ National Vocational Qualification (Scale $0-5)$. 
Table 4.8: Personality Types and Statement "I am good in exams"

\begin{tabular}{lllllll}
\hline & $\begin{array}{l}\text { Female } \\
\text { Applies } \\
\text { very much }\end{array}$ & $\begin{array}{l}\text { Applies } \\
\text { somewhat }\end{array}$ & $\begin{array}{l}\text { Does not } \\
\text { apply }\end{array}$ & $\begin{array}{l}\text { Male } \\
\text { Applies } \\
\text { very much }\end{array}$ & $\begin{array}{l}\text { Applies } \\
\text { somewhat }\end{array}$ & $\begin{array}{l}\text { Does not } \\
\text { apply }\end{array}$ \\
\hline $\begin{array}{l}\text { Personality Type } \\
\text { High Emotional Stability, }\end{array}$ & $19 \%$ & $64 \%$ & $16 \%$ & $28 \%$ & $60 \%$ & $12 \%$ \\
$\begin{array}{l}\text { High Conscientiousness } \\
\text { Low Conscientiousness }\end{array}$ & $6 \%$ & $62 \%$ & $32 \%$ & $11 \%$ & $72 \%$ & $17 \%$ \\
$\begin{array}{l}\text { Low Emotional Stability, } \\
\text { High Conscientiousness }\end{array}$ & $15 \%$ & $64 \%$ & $21 \%$ & $23 \%$ & $59 \%$ & $17 \%$ \\
$\begin{array}{l}\text { Low Emotional Stability, } \\
\text { Low Conscientiousness }\end{array}$ & $7 \%$ & $57 \%$ & $36 \%$ & $13 \%$ & $61 \%$ & $26 \%$ \\
Total & $15 \%$ & $63 \%$ & $22 \%$ & $22 \%$ & $66 \%$ & $16 \%$ \\
\hline
\end{tabular}

Source: BCS70, own calculations based on working sample $\mathrm{n}=2308$. 


\subsubsection{Robustness Checks}

\subsubsection{Ordered Logit Regression Model}

Table 4.9 addresses the relationship between emotional stability and conscientiousness at the ages of 10 and 16 and pessimistic expectations about one's own ability regarding exams at the age of 16 using a generalized ordered logit model. The results are presented following Williams (2006), p.74. It shows that most of the independent variables fit the proportional odds or parallel regression assumption (i.e. no increment of $\log$ odds ratio at cut-off points). However, in the regression model the variable "cognitive ability" does not fit the assumption, which is the reason why two coefficients $(0.337(0.000)$ and $1.783(0.000))$ are presented. This means that scoring higher in cognitive ability significantly increases the likelihood of being in the categories "I am good in exams: applies very much" or "I am good in exams: applies very much or applies somewhat". However, respondents scoring higher in cognitive ability are especially likely to state "I am good in exams: applies very much" $(0.337(0.000))$ as opposed to "I am good in exams: applies very much or applies somewhat" $(0.337(0.000) * 1.783(0.000)=0.601(0.000))$.

The ordinal variable "I am good in exams" is regressed on emotional stability and conscientiousness at the age of 10 as well as a possible personality trait change between the ages of 10 and 16. A personality trait increase (decrease) is considered if the particular trait increases (decreases) by 0.5 of a standard deviation between the ages of 10 and 16. Individuals who do not experience a personality trait change above or below 0.5 of a standard deviation between the ages of 10 and 16 are considered as the reference group. The results are presented as odds ratios. Odds ratios can be interpreted as "the expected number of people with [the expectations "I am good in exams: does not apply"] for every person with [the expectation "I am good in exams: applies very much or applies somewhat"]" (Buis, 2010, p. 306).

The results indicate that both emotional stability and conscientiousness at the age of 10 are significantly negatively related to the expectation of "I am good in exams: does not apply" for girls. The odds for having the expectation of "I am good in exams: does not apply" are 0.86 when emotional stability at the age of 10 increases by 1 standard deviation. This means that in the group of girls who score 1 standard deviation above mean emotional stability, it is to be expected that 0.86 girls have 
negative expectations for every girl with more moderate or positive expectations. Or alternatively, the odds of having the expectation of "I am good in exams: does not apply" decrease by $14 \%$ ( 1 - 0.86) when emotional stability increases by 1 standard deviation. Similarly interpretable, the odds for having the expectation of "I am good in exams: does not apply" are 0.73 when conscientiousness increases by 1 standard deviation. For boys, only conscientiousness at the age of 10 is significantly related to pessimistic expectations. The linear combination of the baseline effect for conscientiousness at the age of 10 and the conscientiousness male interaction effect shows that the odds ratios are 0.84 for boys when conscientiousness increases by 1 standard deviation.

Table 4.9: Personality and Pessimistic Expectations

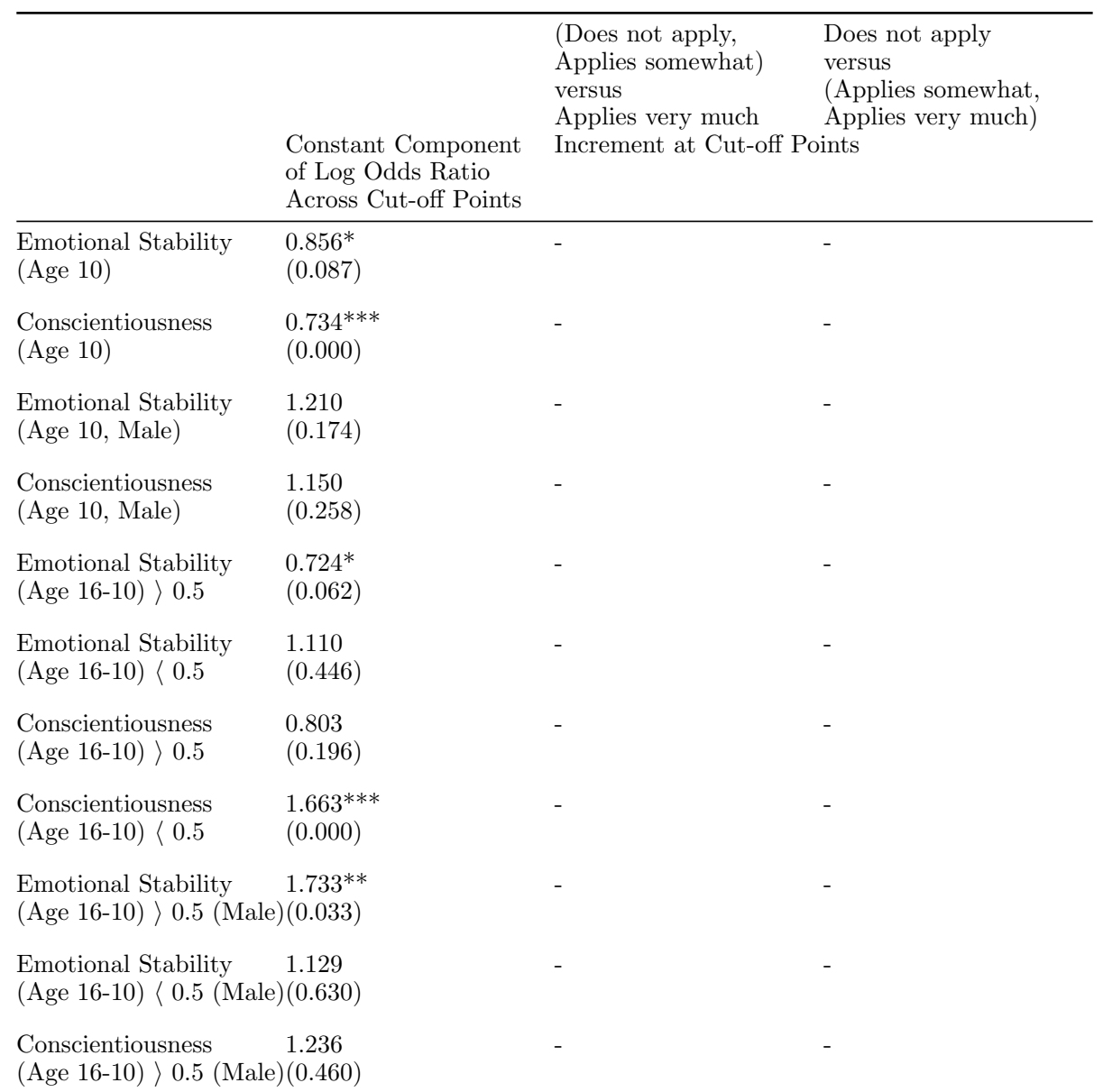




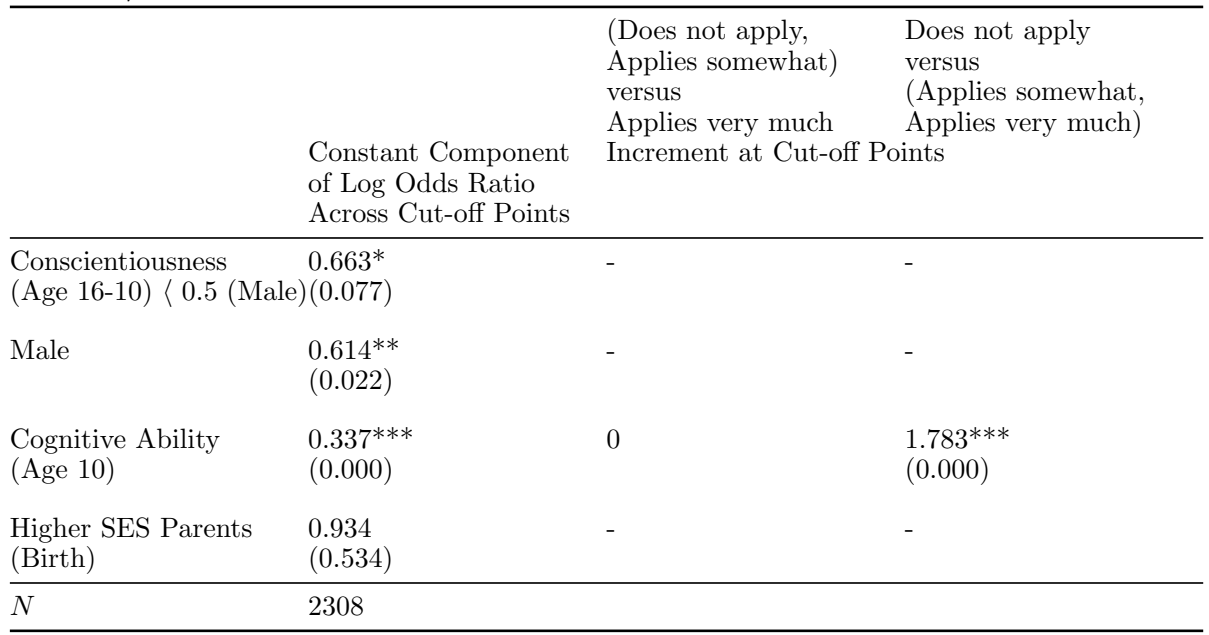

Source: British Cohort Study (BCS70), own calculations. ${ }^{*} p<0.10,{ }^{* *} p<0.05,{ }^{*} * * p<0.01$.

Note: Log odds for unconstrained partial proportional odds model. Generalized ordered logit regression include controls for mother's age, mother's age squared, region of living at birth as well as the personality traits agreeableness and extraversion measured at the ages of 10 and 16 .

For girls, an emotional stability increase between the ages of 10 and 16 is significantly related to less pessimistic expectations regarding one's own ability in exams, while a decrease in conscientiousness between the ages of 10 and 16 is significantly associated to an increase in pessimistic expectations compared to those girls who do not experience a change in the personality traits emotional stability and conscientiousness between the ages of 10 and 16. For boys, there are no significant associations between personality change and pessimistic expectations.

To better understand these associations, predicted probabilities by personality change and gender are estimated. The results are presented in Table 4.10. For girls, the predicted probability to have pessimistic expectations regarding the own ability in exams is decreasing from $22 \%$ to $17 \%$ for girls and from $15 \%$ to $11 \%$ for boys if emotional stability is increasing between the ages of 10 and 16 by more than 0.5 of a standard deviation. The predicted probability to have pessimistic expectations regarding the own ability in exams is increasing from $19 \%$ to $27 \%$ for girls and from $13 \%$ to $19 \%$ for boys if conscientiousness is decreasing between the ages of 10 and 16. 
Table 4.10: Predicted Probabilities for Exam Expectations

\begin{tabular}{lllllll}
\hline \multirow{2}{*}{ Personality Change } & \multicolumn{2}{l}{ Female } & \multicolumn{2}{l}{ Male } \\
\hline Emotional Stability (Age 16-10) $\rangle 0.5=1$ & $12 \%$ & $71 \%$ & $17 \%$ & $18 \%$ & $70 \%$ & $11 \%$ \\
Emotional Stability (Age 16-10) $\rangle 0.5=0$ & $10 \%$ & $69 \%$ & $22 \%$ & $14 \%$ & $71 \%$ & $15 \%$ \\
Conscientiousness (Age 16-10) $\langle 0.5=1$ & $7 \%$ & $66 \%$ & $27 \%$ & $11 \%$ & $70 \%$ & $19 \%$ \\
Conscientiousness (Age 16-10) $\langle 0.5=0$ & $11 \%$ & $70 \%$ & $19 \%$ & $17 \%$ & $70 \%$ & $13 \%$ \\
\hline
\end{tabular}

Source: BCS70, own calculations based on working sample $\mathrm{n}=2308$.

Note: The predicted probability regarding the dependent variable "I am good in exams" refers to the answer categories "p1: applies very much", "p2: applies somewhat" and "p3: does not apply". 


\subsubsection{Imputation of Missing Values}

Table 4.11: Personality and Pessimistic Expectations

\begin{tabular}{|c|c|}
\hline Pessimistic Expectations (Age 16) ... & I am Good in Exams (reverse) \\
\hline Emotional Stability (Age 10) & $\begin{array}{c}-0.039^{*} \\
(0.083)\end{array}$ \\
\hline Conscientiousness (Age 10) & $\begin{array}{l}-0.073^{* * *} \\
(0.001)\end{array}$ \\
\hline Emotional Stability Male (Age 10) & $\begin{array}{l}0.023 \\
(0.499)\end{array}$ \\
\hline Conscientiousness Male (Age 10) & $\begin{array}{l}0.018 \\
(0.548)\end{array}$ \\
\hline Emotional Stability (Age 16-10) $>0.5$ & $\begin{array}{c}-0.082^{*} \\
(0.061)\end{array}$ \\
\hline Emotional Stability (Age 16-10) $<-0.5$ & $\begin{array}{l}0.038 \\
(0.281)\end{array}$ \\
\hline Conscientiousness (Age 16-10) $>0.5$ & $\begin{array}{l}-0.022 \\
(0.617)\end{array}$ \\
\hline Conscientiousness (Age 16-10) $<-0.5$ & $\begin{array}{l}0.151^{* * *} \\
(0.000)\end{array}$ \\
\hline Emotional Stability Male (Age 16-10) $>0.5$ & $\begin{array}{l}0.081 \\
(0.215)\end{array}$ \\
\hline Emotional Stability Male (Age 16-10) $<-0.5$ & $\begin{array}{l}-0.011 \\
(0.861)\end{array}$ \\
\hline Conscientiousness Male (Age 16-10) $>0.5$ & $\begin{array}{l}0.027 \\
(0.697)\end{array}$ \\
\hline Conscientiousness Male (Age 16-10) $<-0.5$ & $\begin{array}{c}-0.105^{*} \\
(0.072)\end{array}$ \\
\hline Male & $\begin{array}{l}-0.107^{* *} \\
(0.049)\end{array}$ \\
\hline Cognitive Ability (Age 10) & $\begin{array}{l}-0.188^{* * *} \\
(0.000)\end{array}$ \\
\hline Higher SES Parents (Birth) & $\begin{array}{l}-0.017 \\
(0.536)\end{array}$ \\
\hline $\begin{array}{l}N \\
R^{2}\end{array}$ & $\begin{array}{l}2614 \\
0.171\end{array}$ \\
\hline
\end{tabular}

Source: British Cohort Study (BCS70), own calculations.

Note: $p$-values in parentheses ${ }^{*} p<0.10,{ }^{* *} p<0.05,{ }^{* * *} p<0.01$. The OLS regressions include controls for mother's age, mother's age squared, region of living at birth as well as the personality traits agreeableness and extraversion measured at the ages of 10 and 16 . 
Table 4.12: Role of Pessimistic Expectations

\begin{tabular}{|c|c|c|c|c|c|c|}
\hline Education Level (Age 30) ... & NVQ $(0-5$ & & Poor $(0,1)$ & & Academic & $(4,5)$ \\
\hline $\begin{array}{l}\text { High Emotional Stability, } \\
\text { Low Conscientiousness } \\
\text { (Age 16) }\end{array}$ & $\begin{array}{l}-0.437 \text { *** } \\
(0.000)\end{array}$ & $\begin{array}{l}-0.344^{* * *} \\
(0.002)\end{array}$ & $\begin{array}{l}0.074 * * \\
(0.031)\end{array}$ & $\begin{array}{l}0.064^{*} \\
(0.065)\end{array}$ & $\begin{array}{l}-0.132^{* * *} \\
(0.001)\end{array}$ & $\begin{array}{l}-0.097^{* *} \\
(0.016)\end{array}$ \\
\hline $\begin{array}{l}\text { Low Emotional Stability, } \\
\text { High Conscientiousness } \\
\text { (Age 16) }\end{array}$ & $\begin{array}{l}-0.209^{* * *} \\
(0.010)\end{array}$ & $\begin{array}{l}-0.174^{* *} \\
(0.028)\end{array}$ & $\begin{array}{l}0.040^{*} \\
(0.068)\end{array}$ & $\begin{array}{l}0.037^{*} \\
(0.095)\end{array}$ & $\begin{array}{l}-0.049 \\
(0.107)\end{array}$ & $\begin{array}{l}-0.035 \\
(0.229)\end{array}$ \\
\hline $\begin{array}{l}\text { Low Emotional Stability, } \\
\text { Low Conscientiousness } \\
\text { (Age 16) }\end{array}$ & $\begin{array}{l}-0.494^{* * *} \\
(0.000)\end{array}$ & $\begin{array}{l}-0.391^{* * *} \\
(0.000)\end{array}$ & $\begin{array}{l}0.091^{* * *} \\
(0.002)\end{array}$ & $\begin{array}{l}0.080 * * * \\
(0.009)\end{array}$ & $\begin{array}{l}-0.140^{* * *} \\
(0.000)\end{array}$ & $\begin{array}{l}-0.101^{* * *} \\
(0.004)\end{array}$ \\
\hline $\begin{array}{l}\text { High Emotional Stability, } \\
\text { Low Conscientiousness } \\
\text { (Male, Age 16) }\end{array}$ & $\begin{array}{l}0.101 \\
(0.517)\end{array}$ & $\begin{array}{l}0.019 \\
(0.904)\end{array}$ & $\begin{array}{l}-0.058 \\
(0.194)\end{array}$ & $\begin{array}{l}-0.048 \\
(0.288)\end{array}$ & $\begin{array}{l}-0.004 \\
(0.945)\end{array}$ & $\begin{array}{l}-0.032 \\
(0.586)\end{array}$ \\
\hline $\begin{array}{l}\text { Low Emotional Stability, } \\
\text { High Conscientiousness } \\
\text { (Male, Age 16) }\end{array}$ & $\begin{array}{l}0.231^{*} \\
(0.086)\end{array}$ & $\begin{array}{l}0.214 \\
(0.105)\end{array}$ & $\begin{array}{l}-0.035 \\
(0.323)\end{array}$ & $\begin{array}{c}-0.034 \\
(0.330)\end{array}$ & $\begin{array}{l}0.017 \\
(0.751)\end{array}$ & $\begin{array}{l}0.010 \\
(0.856)\end{array}$ \\
\hline $\begin{array}{l}\text { Low Emotional Stability, } \\
\text { Low Conscientiousness } \\
\text { (Male, Age 16) }\end{array}$ & $\begin{array}{l}-0.052 \\
(0.734)\end{array}$ & $\begin{array}{l}-0.130 \\
(0.393)\end{array}$ & $\begin{array}{l}-0.017 \\
(0.711)\end{array}$ & $\begin{array}{l}-0.008 \\
(0.859)\end{array}$ & $\begin{array}{l}-0.055 \\
(0.320)\end{array}$ & $\begin{array}{l}-0.084 \\
(0.135)\end{array}$ \\
\hline $\begin{array}{l}\text { Good in Exams: } \\
\text { Applies somewhat } \\
\text { (Age 16) }\end{array}$ & & $\begin{array}{l}-0.569^{* * *} \\
(0.000)\end{array}$ & & $\begin{array}{l}0.046^{* * *} \\
(0.002)\end{array}$ & & $\begin{array}{l}-0.226^{* * *} \\
(0.000)\end{array}$ \\
\hline $\begin{array}{l}\text { Good in Exams: } \\
\text { Does not apply } \\
\text { (Age 16) }\end{array}$ & & $\begin{array}{l}-0.864^{* * *} \\
(0.000)\end{array}$ & & $\begin{array}{l}0.094^{* * *} \\
(0.000)\end{array}$ & & $\begin{array}{l}-0.331^{* * *} \\
(0.000)\end{array}$ \\
\hline $\begin{array}{l}\text { Good in Exams: } \\
\text { Applies somewhat } \\
\text { (Male, Age } 16 \text { ) }\end{array}$ & & $\begin{array}{l}0.421^{\text {*** }} \\
(0.000)\end{array}$ & & $\begin{array}{l}-0.054^{* *} \\
(0.016)\end{array}$ & & $\begin{array}{l}0.125^{* * *} \\
(0.007)\end{array}$ \\
\hline $\begin{array}{l}\text { Good in Exams: } \\
\text { Does not apply } \\
\text { (Male, Age } 16 \text { ) }\end{array}$ & & $\begin{array}{l}0.234 \\
(0.130)\end{array}$ & & $\begin{array}{l}-0.006 \\
(0.895)\end{array}$ & & $\begin{array}{l}0.103^{*} \\
(0.076)\end{array}$ \\
\hline Male & $\begin{array}{l}0.138^{* *} \\
(0.042)\end{array}$ & $\begin{array}{l}-0.193^{* *} \\
(0.043)\end{array}$ & $\begin{array}{c}-0.029 * \\
(0.085)\end{array}$ & $\begin{array}{l}0.009 \\
(0.634)\end{array}$ & $\begin{array}{l}0.042 \\
(0.123)\end{array}$ & $\begin{array}{l}-0.065 \\
(0.122)\end{array}$ \\
\hline $\begin{array}{l}\text { Cognitive Ability } \\
\text { (Age 10) }\end{array}$ & $\begin{array}{l}0.470^{* * *} \\
(0.000)\end{array}$ & $\begin{array}{l}0.396^{* * *} \\
(0.000)\end{array}$ & $\begin{array}{l}-0.088^{* * *} \\
(0.000)\end{array}$ & $\begin{array}{l}-0.080^{* * *} \\
(0.000)\end{array}$ & $\begin{array}{l}0.141^{* * *} \\
(0.000)\end{array}$ & $\begin{array}{l}0.113^{* * *} \\
(0.000)\end{array}$ \\
\hline $\begin{array}{l}\text { Higher SES Parents } \\
\text { (Birth) }\end{array}$ & $\begin{array}{l}0.374^{* * *} \\
(0.000)\end{array}$ & $\begin{array}{l}0.365^{* * *} \\
(0.000)\end{array}$ & $\begin{array}{l}-0.047^{* * *} \\
(0.000)\end{array}$ & $\begin{array}{l}-0.047^{* * *} \\
(0.000)\end{array}$ & $\begin{array}{l}0.145^{* * *} \\
(0.000)\end{array}$ & $\begin{array}{l}0.141^{* * *} \\
(0.000)\end{array}$ \\
\hline
\end{tabular}


... Table 4.12 continued

\begin{tabular}{lllllll}
\hline Education Level (Age 30) $\ldots$ & NVQ (0-5) & Poor $(0,1)$ & Academic $(4,5)$ \\
\hline$N$ & 2614 & 2614 & 2614 & 2614 & 2614 & 2614 \\
$R^{2}$ & 0.252 & 0.278 & 0.116 & 0.124 & 0.191 & 0.218 \\
\hline
\end{tabular}

Source: British Cohort Study (BCS70), own calculations.

Note: $p$-values in parentheses ${ }^{*} p<0.10,{ }^{* *} p<0.05,{ }^{* * *} p<0.01$. Poor and academic education are binary variables, whereby the values in brackets are coded as 1 . The reference group for the personality types comprises more emotionally stable and more conscientious individuals. The reference group for the exam expectations are individuals who respond that the statement "I am good in exams" applies very much. The OLS regressions include controls for mother's age, mother's age squared, region of living at birth as well as the personality traits agreeableness and extraversion measured at the age of 16 .

Table 4.13: Interaction between Personality and Pessimistic Expectations

\begin{tabular}{|c|c|c|c|}
\hline Education Level (Age 30) ... & NVQ (0-5) & Poor $(0,1)$ & Academic $(4,5)$ \\
\hline $\begin{array}{l}\text { High Emotional Stability, High Conscientiousness } \\
\text { Good in Exams: Applies very much, somewhat } \\
\text { (Age 16) }\end{array}$ & $\begin{array}{l}0.706^{* * *} \\
(0.000)\end{array}$ & $\begin{array}{l}-0.121^{* *} \\
(0.011)\end{array}$ & $\begin{array}{l}0.209^{* * * *} \\
(0.000)\end{array}$ \\
\hline $\begin{array}{l}\text { High Emotional Stability, High Conscientiousness } \\
\text { Good in Exams: Does not apply (Age 16) }\end{array}$ & $\begin{array}{l}0.397^{* *} \\
(0.016)\end{array}$ & $\begin{array}{l}-0.088 \\
(0.109)\end{array}$ & $\begin{array}{l}0.083 \\
(0.137)\end{array}$ \\
\hline $\begin{array}{l}\text { High Emotional Stability, Low Conscientiousness } \\
\text { Good in Exams: Applies very much, somewhat } \\
\text { (Age 16) }\end{array}$ & $\begin{array}{l}0.366^{* *} \\
(0.039)\end{array}$ & $\begin{array}{l}-0.045 \\
(0.439)\end{array}$ & $\begin{array}{l}0.121^{* *} \\
(0.042)\end{array}$ \\
\hline $\begin{array}{l}\text { High Emotional Stability, Low Conscientiousness } \\
\text { Good in Exams: Does not apply (Age 16) }\end{array}$ & $\begin{array}{l}-0.169 \\
(0.412)\end{array}$ & $\begin{array}{l}-0.024 \\
(0.753)\end{array}$ & $\begin{array}{l}-0.110^{*} \\
(0.081)\end{array}$ \\
\hline $\begin{array}{l}\text { Low Emotional Stability, High Conscientiousness } \\
\text { Good in Exams: Applies very much, somewhat } \\
\text { (Age 16) }\end{array}$ & $\begin{array}{l}0.562^{* * *} \\
(0.000)\end{array}$ & $\begin{array}{l}-0.104^{* *} \\
(0.032)\end{array}$ & $\begin{array}{l}0.175^{* * *} \\
(0.000)\end{array}$ \\
\hline $\begin{array}{l}\text { Low Emotional Stability, High Conscientiousness } \\
\text { Good in Exams: Does not apply (Age 16) }\end{array}$ & $\begin{array}{l}-0.036 \\
(0.853)\end{array}$ & $\begin{array}{l}0.045 \\
(0.517)\end{array}$ & $\begin{array}{l}-0.005 \\
(0.929)\end{array}$ \\
\hline $\begin{array}{l}\text { Low Emotional Stability, Low Conscientiousness } \\
\text { Good in Exams: Applies very much, somewhat } \\
\text { (Age 16) }\end{array}$ & $\begin{array}{l}0.225 \\
(0.137)\end{array}$ & $\begin{array}{l}-0.032 \\
(0.538)\end{array}$ & $\begin{array}{l}0.068 \\
(0.160)\end{array}$ \\
\hline $\begin{array}{l}\text { High Emotional Stability, High Conscientiousness } \\
\text { Good in Exams: Applies very much, somewhat } \\
\text { (Male, Age 16) }\end{array}$ & $\begin{array}{l}0.357 \\
(0.193)\end{array}$ & $\begin{array}{l}-0.105 \\
(0.231)\end{array}$ & $\begin{array}{l}0.084 \\
(0.317)\end{array}$ \\
\hline $\begin{array}{l}\text { High Emotional Stability, High Conscientiousness } \\
\text { Good in Exams: Does not apply (Male, Age 16) }\end{array}$ & $\begin{array}{l}0.019 \\
(0.954)\end{array}$ & $\begin{array}{l}-0.011 \\
(0.919)\end{array}$ & $\begin{array}{l}0.003 \\
(0.974)\end{array}$ \\
\hline
\end{tabular}


... Table 4.13 continued

\begin{tabular}{|c|c|c|c|}
\hline Education Level (Age 30) ... & NVQ $(0-5)$ & Poor $(0,1)$ & Academic $(4,5)$ \\
\hline $\begin{array}{l}\text { High Emotional Stability, Low Conscientiousness } \\
\text { Good in Exams: Applies very much, somewhat } \\
\text { (Male, Age 16) }\end{array}$ & $\begin{array}{l}0.282 \\
(0.353)\end{array}$ & $\begin{array}{l}-0.142 \\
(0.138)\end{array}$ & $\begin{array}{l}0.011 \\
(0.909)\end{array}$ \\
\hline $\begin{array}{l}\text { High Emotional Stability, Low Conscientiousness } \\
\text { Good in Exams: Does not apply (Male, Age 16) }\end{array}$ & $\begin{array}{l}0.701^{*} \\
(0.081)\end{array}$ & $\begin{array}{l}-0.175 \\
(0.165)\end{array}$ & $\begin{array}{l}0.203^{*} \\
(0.097)\end{array}$ \\
\hline $\begin{array}{l}\text { Low Emotional Stability, High Conscientiousness } \\
\text { Good in Exams: Applies very much, somewhat } \\
\text { (Male, Age 16) }\end{array}$ & $\begin{array}{l}0.513^{*} \\
(0.065)\end{array}$ & $\begin{array}{l}-0.099 \\
(0.266)\end{array}$ & $\begin{array}{l}0.087 \\
(0.319)\end{array}$ \\
\hline $\begin{array}{l}\text { Low Emotional Stability, High Conscientiousness } \\
\text { Good in Exams: Does not apply (Male, Age 16) }\end{array}$ & $\begin{array}{l}0.681^{*} \\
(0.073)\end{array}$ & $\begin{array}{l}-0.245^{* *} \\
(0.037)\end{array}$ & $\begin{array}{l}0.113 \\
(0.361)\end{array}$ \\
\hline $\begin{array}{l}\text { Low Emotional Stability, Low Conscientiousness } \\
\text { Good in Exams: Applies very much, somewhat } \\
\text { (Male, Age 16) }\end{array}$ & $\begin{array}{l}0.308 \\
(0.269)\end{array}$ & $\begin{array}{l}-0.135 \\
(0.136)\end{array}$ & $\begin{array}{l}0.017 \\
(0.840)\end{array}$ \\
\hline Male & $\begin{array}{l}-0.187 \\
(0.467)\end{array}$ & $\begin{array}{l}0.065 \\
(0.443)\end{array}$ & $\begin{array}{l}-0.037 \\
(0.627)\end{array}$ \\
\hline $\begin{array}{l}N \\
R^{2}\end{array}$ & $\begin{array}{l}2614 \\
0.268\end{array}$ & $\begin{array}{l}2614 \\
0.127\end{array}$ & $\begin{array}{l}2614 \\
0.204\end{array}$ \\
\hline
\end{tabular}

Source: British Cohort Study (BCS70), own calculations.

Note: $p$-values in parentheses $* p<0.10,{ }^{*} * p<0.05, * * * p<0.01$. Poor and academic education are binary variables, whereby the values in brackets are coded as 1 . The reference group for the personality types comprises less emotionally stable and less conscientious individuals who have pessimistic exam expectations. The OLS regressions include controls for mother's age, mother's age squared, region and parental socioeconomic background at birth, the personality traits agreeableness and extraversion measured at the age of 16 as well as cognitive ability measured at the age of 10 . 


\section{CHAPTER 5}

\section{PERSONALITY AND}

\section{MENTAL HEALTH*}

\footnotetext{
* Chapters 5 and 6 are based on joined work with Lex Borghans and Trudie Schils. The author particularly thanks Christopher Boyce, Deborah Cobb-Clark, Arnaud Chevalier, Bart Golsteyn, Peter Kuhn, Oliver Lüdtke, Milena Nikolova, Brent W. Roberts and Ulf Zölitz for valuable comments. The chapters have been presented at the conferences "Economics, Health and Happiness" in Lugano, at the "19th IZA European Summer School in Labor Economics", at the "2016 Stirling PhD Conference in Behavioral Science" as well as at Maastricht University where the author received helpful input from the participants. This chapter is related to the IZA Discussion Paper 10337 "Personality and Mental Health: The Role and Substitution Effect of Emotional Stability and Conscientiousness" that is currently under review.
} 


\subsection{Introduction}

Do the observed associations between emotional stability, conscientiousness and educational achievement also hold when looking at the subjective measure mental health? If some individuals choose less efficient or effective strategies in handling challenging situation, but feel comfortable with the consequences, just variation in preferences is observed. However, if the patterns are similar, individuals at-risk are likely to face a multidimensional disadvantage. This question is addressed as follows: (1) The predictive power of low emotional stability at the age of 16 for mental health problems at the ages of $16,26,30,34$ and 42 as well as the possible substitution effect of conscientiousness is analyzed. (2) The relationship between a change in emotional stability and conscientiousness between the ages of 10 and 16 and mental ill-health in later age is estimated.

The theory presented in Chapter 2 predicts that less emotionally stable individuals have a higher stress-reactivity in challenging situations and are more likely to avoid problems rather than solving them due to negative emotion. However, if the challenge is unavoidable such as an exam in school, it is likely to persist. This might result in feelings of sustained stress and mental health problems. Conscientiousness is expected to mitigate the positive association between low emotional stability and mental health problems, because conscientious individuals are more likely to apply problem-focused strategies. This might be related to a boost in control over the challenging situation and a reduction in arousal. Moreover, the probability of making positive experiences is more likely which contributes to perceptions of success and mastering.

Given that personality traits are considered mostly rank-order stable at adult age (Roberts and Del Vecchio, 2000; Borghans et al., 2008), it is hypothesized that the relation between emotional stability and mental health outcomes remains relatively robust throughout life, because individuals at-risk are likely to be trapped in unhealthy expectation, feeling and behavior patterns.

Chapter 3 shows that particularly women who score low in both emotional stability and conscientiousness at the age of 16 have a significantly higher risk of only reaching poor education outcomes at the age of 30 even after controlling for cognitive ability and socioeconomic background. For men, conscientiousness in adolescence seems to be more important regarding educational achievement at the age of 30 . 
It is interesting to know if there are similar associations regarding mental health outcomes.

Economic studies have explored various risks for mental health related to negative life experiences such as family characteristics (Johnston et al., 2013; Layard et al., 2014; Persson and Rossin-Slater, 2016), working conditions (Karasek, 1979; Karasek and Theorell, 1992; Siegrist, 1996; Bardasi and Francesconi, 2004; Straathof and Weehuizen, 2005; Cottini and Lucifora, 2013; Hudson and Barrett, 2014; Drydakis, 2015) or environmental shocks (Lindeboom et al., 2002; Cesur et al., 2013; Cornaglia et al., 2014; Dustmann and Fasani, 2016; Danzer and Danzer, 2016).

The chapter builds on economic literature that targets intrinsic risks for psychological and physical health related to variation in personality (Cobb-Clark et al., 2014; Mendolia and Walker, 2014a; Blázquez Cuesta and Budría, 2015). However, these articles do not provide and test a coherent framework of possible mechanisms for the relation between personality and mental health outcomes in a longitudinal study. In the next sections, the data are introduced and the results are discussed. The final section concludes.

\subsection{Data}

The analysis is based on the BCS70, which is conducted by the Center for Longitudinal Studies (CLS) at the Department for Quantitative Social Science in the Institute of Education at London University and follows the lives of 17,196 people born in England, Scotland and Wales during one week in 1970. The BCS70 provides detailed information about the individual's family background, cognitive ability, personality and indicators of mental health problems.

Chapter 3 Appendix Table 3.6 provides an overview of the BCS70 datasets used in the analysis, the variables investigated and the overall number of observations in each of the eight waves. The initial number of observations presented in Chapter 3 Appendix Table 3.6 is not congruent with the actual number of observations used in the analysis due to panel attrition and missing values within the variables of interest. The working sample is restricted to cases with available information on the personality traits emotional stability, conscientiousness, agreeableness and extraversion at the ages of 10 and 16, cognitive ability at the age of 10, mother's age, parental socioeconomic status and region of living at birth as well as one mental 
ill-health score at a time, resulting in five separate samples with 2559 (age 16), 3436 (age 26), 4200 (age 30), 3789 (age 34) and 3369 (age 42) observations.

The issue of sample selection is addressed by descriptively comparing the working samples with the unrestricted samples (Appendix Subsection 5.5.1). The comparison shows that the working samples are positively selected. This makes it likely to estimate lower bound results and that the observed associations are even larger in the unrestricted sample. For a more detailed discussion see Chapter 3 Subsection 3.2 .

To test the robustness of the results, two checks are done. First, all calculations are repeated based on a fully restricted sample, which restricts the availability of all variables used and includes 1,270 observations. The results are consistent with the main results and are available in Appendix Subsection 5.5.2.2. Second, multiple imputation of missing values regarding the personality trait variables and the indicator of mental health problems is conducted. Personality items are imputed if there are five or fewer missing values out of 22 items. Malaise items are imputed if there are three or fewer missing values out of nine items. Imputation of missing values results in an increase of the working samples' size to 3142 (age 16), 4066 (age 26), 4910 (age 30), 4399 (age 34) and 3878 (age 42) observations. The results are consistent with the main results and available in Appendix Subsection 5.5.2.3. In the following, all variables are discussed in detail.

\subsubsection{Mental Health Outcomes}

An indicator of mental health problems is assessed by Rutter et al. (1970)'s Malaise Inventory, which is measured at the ages of 16, 26, 30, 34 and 42 and that covers emotional disturbance and associated physical symptoms (UK Data Service, 2016). The Malaise Inventory is based on the Cornell Medical Index Health Questionnaire (CMI), which comprises 195 self-completion questions (Brodman et al., 1949, 1952).

The internal consistency and validity of the Malaise Inventory has been tested and shown to hold in various socioeconomic groups (Rodgers et al., 1999) and has been used in many studies (Rutter and Madge, 1976; McGee et al., 1986; Grant et al., 1990). Rutter et al. (1970) confirm that "the inventory differentiates moderately well between individuals with and without psychiatric disorder" (p. 160, see UK Data Service, 2016). The Malaise Inventory includes questions such as "Do you feel tired 
Figure 5.1: Mental Ill-Health at Ages 16, 26, 30, 34 and 42

Age 16

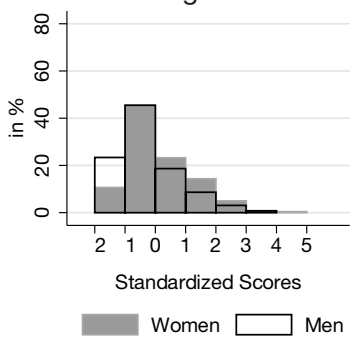

Age 34

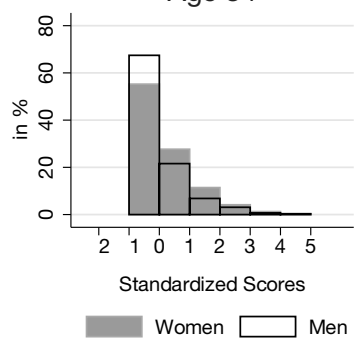

Age 26

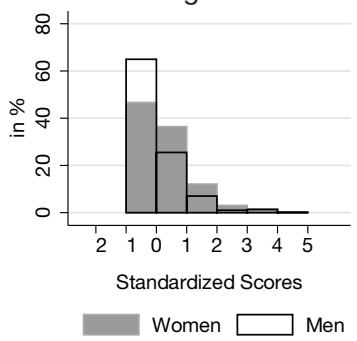

Age 42

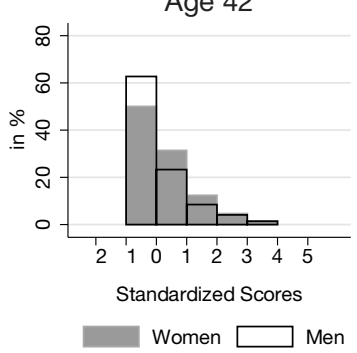

Age 30

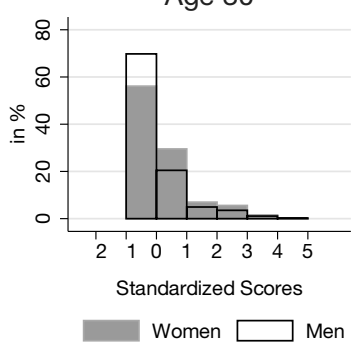

Source: BCS70. Own calculations, $\mathrm{n}=2559$ (age 16), $\mathrm{n}=3436$ (age 26), $\mathrm{n}=4200$ (age 30), $\mathrm{n}=$ 3789 (age 34 ), $\mathrm{n}=3369$ (age 42).

most of the time?", "Do you often feel depressed?" and "Do you often get worried about things?" Higher item values indicate more severe problems. The individual malaise score by wave is generated by summing up the responses to the item set.

Both the number of items within the item set and the measurement scale vary across waves. At the age of 16, the item set includes 22 items, whereby the malaise items are measured on a three-point scale with the categories 0 "rarely or never," 1 "some of the time" and 2 "most of the time." At the ages of 26 and 30, the item set includes 24 items, while at the ages of 34 and 42 it includes only nine items. At the ages of $26,30,34$ and 42 , the malaise items are measured on a two-point scale with the categories 0 "no" and 1 "yes."

Appendix Table 5.13 provides an overview of the questions, the availability of each item at different ages, the measurement scales for the items, the measurement scales for the sum scores and the threshold that is regarded as a sign that the respondent experiences symptoms related to depression. To make the mental ill-health variable 
comparable between different ages, the score for mental health problems is restricted to the nine questions that are available in all five waves. Therefore, the questions used are the items 2, 3, 5, 9, 12, 14, 16, 20 and 21 that are marked in bold in Appendix Table 5.13. Only individuals who have no missing values in the nine items are taken into account. Standardized mental ill-health scores are used in the analysis.

Figure 5.1 depicts the distribution of mental health problems. It shows that females are more often affected by symptoms of mental ill-health than males at all ages and that, on average, mental health problems decrease in early adult age. This result is in accordance with the psychology literature. Table 5.1 shows the correlation coefficients for the mental ill-health scores at the ages of 16, 26, 30, 34 and 42. The size of the correlation coefficients varies between 0.26 and 0.57 . The results show that mental health problems seem to be more malleable in younger age, while they seem to be more stable at older age: The correlation between mental health problems at the ages of 16 and 26 is only 0.39 . It is 0.56 or 0.57 between the ages of 26 and 30, the ages of 30 and 34 as well as the ages of 34 and 42 .

Table 5.1: Correlation of Mental Ill-Health Scores

\begin{tabular}{lllll}
\hline & Age 16 & Age 26 & Age 30 & Age 34 \\
\hline Age 26 & 0.39 & - & - & - \\
Age 30 & 0.35 & 0.57 & - & - \\
Age 34 & 0.35 & 0.50 & 0.56 & - \\
Age 42 & 0.26 & 0.47 & 0.48 & 0.57 \\
\hline
\end{tabular}

BCS70. The sample applied is the fully restricted working sample $(\mathrm{n}=1270)$.

Note: Scores are standardized. Correlation coefficients are significant at the $5 \%$ level or higher.

\subsubsection{Personality Traits}

In the analysis, personality traits at the ages of 10 and 16 are used threefold: First, they are used as continues variables as shown in Figure 5.2. Second, four personality types capturing differences in emotional stability and conscientiousness are created. Thereby, the distribution of the standardized variables emotional stability and conscientiousness is split at the mean, i.e. at zero, and four above- and below-mean combinations of emotional stability and conscientiousness are constructed: (1) more emotionally stable and more conscientious, (2) more emotionally stable and less 
conscientious, (3) less emotionally stable and more conscientious, and (4) less emotionally stable and less conscientious individuals. Third, the change in personality between the ages of 10 and 16 is used by subtracting the standardized personality score at the age of 10 from the standardized score at the age of 16 .

Figure 5.2: Personality Traits at Ages 10 and 16
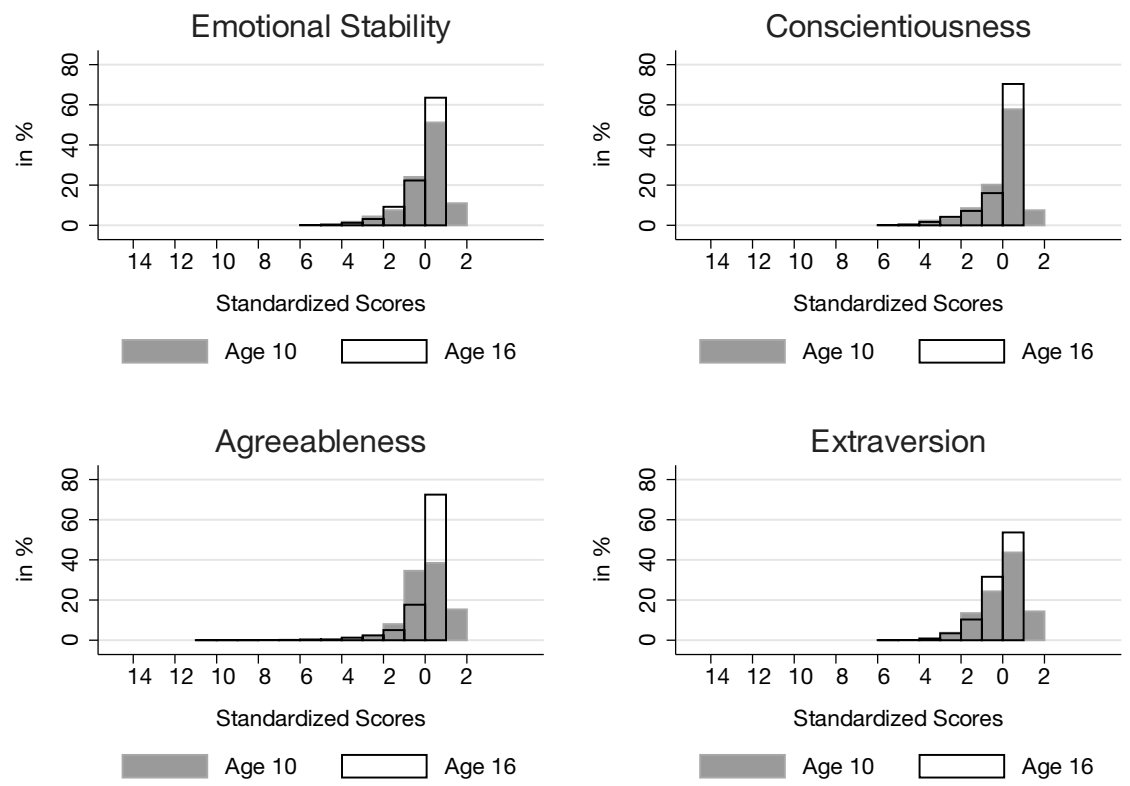

Source: BCS70. Own calculations, $\mathrm{n}=2559$.

A detailed discussion about the personality traits used in the analysis is provided in Chapter 3 Subsection 3.2.2. In this chapter, only the number of observations varies. An overview of the reliability measures that correspond to the four personality traits is provided in Appendix Table 5.14. The distribution of emotional stability, conscientiousness, agreeableness and extraversion is presented in Figure 5.2. An overview of the correlation between the personality trait variables, the behavioral items that are used to construct the personality trait variables and the five scores for mental ill-health is provided in Appendix Table 5.15. It becomes obvious that the correlation coefficients between low emotional stability as well as the related behavioral items and mental ill-health are the largest. 


\subsubsection{Additional Controls}

In addition to the variables discussed above, controls are included for the individual's cognitive ability at the age of 10 and the individual family background at birth. Detailed information is provided in Chapter 3 Subsection 3.2.3.

\section{$5.3 \quad$ Results}

The results are based on OLS regression methods. All regressions focus on the relation between emotional stability and conscientiousness, and mental health outcomes in adolescence and adulthood. In all of the regressions, controls are included for the respondent's cognitive ability, the personality traits extraversion and agreeableness, parental socioeconomic background and region of living at birth as well as the mother's age. To account for the possible problem of heteroscedasticity, robust standard errors are calculated.

\subsubsection{Personality and Mental Ill-Health}

Table 5.2 presents the results regarding the predictive power of emotional stability and conscientiousness at the age of 16 for mental health problems at the ages of 16 , 26, 30, 34 and 42. The results reveal that emotional stability significantly predicts mental health in adolescence and adulthood and that the size of the coefficients remains relatively stable over time. An increase in emotional stability by 1 standard deviation is related to a decrease in mental health problems by between 0.08 and 0.17 of a standard deviation. Conscientiousness at the age of 16 is negatively related to mental health problems, although the coefficients are less robust over time than those for emotional stability.

It is further shown that women are more often affected by mental ill-health than men at all ages. On average, men score between 0.19 and 0.35 of a standard deviation lower on mental health problems compared to women. The gender gap is decreasing with age. This result is in accordance with the psychological literature about gender differences regarding depressive symptoms (Hankin et al., 1998; Broidy et al., 2003). For instance, Broidy et al. (2003) find that girls who show disruptive behavior at young age experience more often internal behavior problems such as depression 
later in life, while boys with behavior problems exhibit more often external behavior problems like physical aggression in older age. Shown in Appendix Table 5.13, the nine malaise items used in the analysis cover mostly depression related symptoms rather than aggression related symptoms.

Table 5.2: Role of Emotional Stability and Conscientiousness

\begin{tabular}{llllll}
\hline Mental Ill-Health Score $\ldots$ & Age 16 & Age 26 & Age 30 & Age 34 & Age 42 \\
\hline Emotional Stability (Age 16) & $-0.166^{* * *}$ & $-0.109^{* * *}$ & $-0.118^{* * *}$ & $-0.084^{* * *}$ & $-0.115^{* * *}$ \\
& $(0.000)$ & $(0.000)$ & $(0.000)$ & $(0.000)$ & $(0.000)$ \\
Conscientiousness (Age 16) & -0.028 & $-0.058^{* * *}$ & $-0.070^{* * *}$ & $-0.087^{* * *}$ & -0.015 \\
& $(0.256)$ & $(0.005)$ & $(0.000)$ & $(0.000)$ & $(0.479)$ \\
Male & $-0.329^{* * *}$ & $-0.346^{* * *}$ & $-0.250^{* * *}$ & $-0.239^{* * *}$ & $-0.192^{* * *}$ \\
& $(0.000)$ & $(0.000)$ & $(0.000)$ & $(0.000)$ & $(0.000)$ \\
\hline$N$ & 2559 & 3436 & 4200 & 3789 & 3369 \\
$R^{2}$ & 0.104 & 0.101 & 0.069 & 0.068 & 0.063 \\
\hline
\end{tabular}

Source: British Cohort Study (BCS70), own calculations.

Note: $p$-values in parentheses ${ }^{*} p<0.10,{ }^{* *} p<0.05,{ }^{* * *} p<0.01$. The OLS regressions include controls for mother's age, mother's age squared, region of living and parental socioeconomic background at birth, the personality traits agreeableness and extraversion measured at the age of 16 as well as cognitive ability measured at the age of 10 .

In Tables 5.3 and 5.4, the substitution effect of conscientiousness for the relationship between low emotional stability and mental health problems is analyzed. It is hypothesizes that at any level of emotional stability, mental ill-health is less of concern with increasing conscientiousness. This means that conscientiousness is able to buffer the positive relation between low emotional stability and mental health problems, because it is likely to compensate for the reduced problem-solving ability and sustained arousal related to low emotional stability.

Table 5.3 shows a borderline significant negative interaction effect between the continuous variables emotional stability and conscientiousness for women in adult age. This means that the negative association between emotional stability and mental health problems is stronger with increasing conscientiousness. Accordingly, conscientiousness is likely to mitigate the positive relationship between low emotional stability and mental ill-health which is in line with the theoretical considerations (see also Appendix Table 5.26). However, for men, no interaction effects are found. 
Table 5.3: Substitution Effect of Conscientiousness

\begin{tabular}{|c|c|c|c|c|c|}
\hline Mental Ill-Health Score ... & Age 16 & Age 26 & Age 30 & Age 34 & Age 42 \\
\hline Emotional Stability (Age 16) & $\begin{array}{l}-0.194^{* * *} \\
(0.000)\end{array}$ & $\begin{array}{l}-0.091^{* * *} \\
(0.001)\end{array}$ & $\begin{array}{l}-0.129^{* * *} \\
(0.000)\end{array}$ & $\begin{array}{l}-0.098^{* *} \\
(0.001)\end{array}$ & $\begin{array}{l}-0.139 * * * \\
(0.000)\end{array}$ \\
\hline Conscientiousness (Age 16) & $\begin{array}{l}-0.017 \\
(0.622)\end{array}$ & $\begin{array}{l}-0.080^{* *} \\
(0.014)\end{array}$ & $\begin{array}{l}-0.085^{* *} \\
(0.011)\end{array}$ & $\begin{array}{l}-0.105^{* * *} \\
(0.004)\end{array}$ & $\begin{array}{l}-0.038 \\
(0.216)\end{array}$ \\
\hline $\begin{array}{l}\text { Emotional Stability } \\
\text { Male (Age 16) }\end{array}$ & $\begin{array}{l}0.065 \\
(0.222)\end{array}$ & $\begin{array}{l}-0.037 \\
(0.382)\end{array}$ & $\begin{array}{l}0.013 \\
(0.745)\end{array}$ & $\begin{array}{l}0.034 \\
(0.428)\end{array}$ & $\begin{array}{l}0.054 \\
(0.206)\end{array}$ \\
\hline $\begin{array}{l}\text { Conscientiousness } \\
\text { Male (Age 16) }\end{array}$ & $\begin{array}{l}-0.031 \\
(0.526)\end{array}$ & $\begin{array}{l}0.048 \\
(0.251)\end{array}$ & $\begin{array}{l}0.011 \\
(0.782)\end{array}$ & $\begin{array}{l}0.017 \\
(0.706)\end{array}$ & $\begin{array}{l}0.027 \\
(0.535)\end{array}$ \\
\hline $\begin{array}{l}\text { Emotional Stability } \times \\
\text { Conscientiousness (Age 16) }\end{array}$ & $\begin{array}{l}-0.002 \\
(0.903)\end{array}$ & $\begin{array}{l}0.001 \\
(0.943)\end{array}$ & $\begin{array}{l}-0.016 \\
(0.297)\end{array}$ & $\begin{array}{l}-0.025 \\
(0.172)\end{array}$ & $\begin{array}{l}-0.023 \\
(0.151)\end{array}$ \\
\hline $\begin{array}{l}\text { Emotional Stability } \times \\
\text { Conscientiousness } \times \\
\text { Male (Age 16) }\end{array}$ & $\begin{array}{l}-0.012 \\
(0.752)\end{array}$ & $\begin{array}{l}0.004 \\
(0.900)\end{array}$ & $\begin{array}{l}0.002 \\
(0.929)\end{array}$ & $\begin{array}{l}0.042 \\
(0.132)\end{array}$ & $\begin{array}{l}0.026 \\
(0.395)\end{array}$ \\
\hline Male & $\begin{array}{l}-0.327^{* * *} \\
(0.000)\end{array}$ & $\begin{array}{l}-0.346^{* * *} \\
(0.000)\end{array}$ & $\begin{array}{l}-0.254^{* * *} \\
(0.000)\end{array}$ & $\begin{array}{l}-0.261^{* * *} \\
(0.000)\end{array}$ & $\begin{array}{l}-0.209^{* * *} \\
(0.000)\end{array}$ \\
\hline $\begin{array}{l}N \\
R^{2}\end{array}$ & $\begin{array}{l}2559 \\
0.106\end{array}$ & $\begin{array}{l}3436 \\
0.101\end{array}$ & $\begin{array}{l}4200 \\
0.069\end{array}$ & $\begin{array}{l}3789 \\
0.068\end{array}$ & $\begin{array}{l}3369 \\
0.065\end{array}$ \\
\hline
\end{tabular}

Source: British Cohort Study (BCS70), own calculations.

Note: $p$-values in parentheses ${ }^{*} p<0.10,{ }^{* *} p<0.05,{ }^{* * *} p<0.01$. The OLS regressions include controls for mother's age, mother's age squared, region of living and parental socioeconomic background at birth, the personality traits agreeableness and extraversion measured at the age of 16 as well as cognitive ability measured at the age of 10 .

In Table 5.4, the substitution effect of conscientiousness is further illuminated by including four personality types comprising (1) more emotionally stable and more conscientious, (2) more emotionally stable and less conscientious, (3) less emotionally stable and more conscientious and (4) less emotionally stable and less conscientious individuals into the regression. The reference group comprises more emotionally stable and more conscientious individuals, i.e. theoretically considered as the most favorable combination. 
Table 5.4: Personality Types and Mental Ill-Health

\begin{tabular}{llllll}
\hline Mental Ill-Health Score $\ldots$ & Age 16 & Age 26 & Age 30 & Age 34 & Age 42 \\
\hline High Emotional Stability, & 0.092 & 0.054 & $0.142^{* * *}$ & $0.085^{*}$ & -0.012 \\
Low Conscientiousness (Age 16) & $(0.147)$ & $(0.297)$ & $(0.003)$ & $(0.079)$ & $(0.818)$ \\
Low Emotional Stability, & $0.251^{* * *}$ & 0.067 & $0.113^{* *}$ & $0.096^{* *}$ & $0.154^{* * *}$ \\
High Conscientiousness (Age 16) & $(0.000)$ & $(0.151)$ & $(0.011)$ & $(0.039)$ & $(0.002)$ \\
Low Emotional Stability, & $0.305^{* * *}$ & $0.263^{* * *}$ & $0.338^{* * *}$ & $0.310^{* * *}$ & $0.245^{* * *}$ \\
Low Conscientiousness (Age 16) & $(0.000)$ & $(0.000)$ & $(0.000)$ & $(0.000)$ & $(0.000)$ \\
Male & $-0.343^{* * *}$ & $-0.356^{* * *}$ & $-0.263^{* * *}$ & $-0.242^{* * *}$ & $-0.198^{* * *}$ \\
& $(0.000)$ & $(0.000)$ & $(0.000)$ & $(0.000)$ & $(0.000)$ \\
\hline$N$ & 2559 & 3436 & 4200 & 3789 & 3369 \\
$R^{2}$ & 0.097 & 0.096 & 0.066 & 0.065 & 0.062 \\
\hline
\end{tabular}

Source: British Cohort Study (BCS70), own calculations.

Note: $p$-values in parentheses ${ }^{*} p<0.10,{ }^{* *} p<0.05,{ }^{* * *} p<0.01$. The reference group for the personality types comprises emotionally stable and highly conscientious individuals. The OLS regressions include controls for mother's age, mother's age squared, region of living and parental socioeconomic background at birth, the personality traits agreeableness and extraversion measured at the age of 16 as well as cognitive ability measured at the age of 10 .

The analysis shows that less emotionally stable and less conscientious individuals have the highest risk of experiencing mental health problems at young and older age. This personality type is related to an increase in mental ill-health by between 0.25 and 0.34 of a standard deviation compared to more emotionally stable and more conscientious individuals.

This result is in line with the theoretical considerations in Chapter 2. Accordingly, less emotionally stable and less conscientious individuals are more likely to have a reduced perceived and actual problem-solving ability related to pessimistic expectations and emotion-focused coping strategies, including in influenceable challenging situations throughout life. This is likely to be related to feelings of sustained stress, an increase in anxiety and worse outcomes because the underlying problem mentally and actually persists. In the long run, mental ill-health might arise and endure.

More conscientiousness is expected to mitigate this positive relation. Indeed, less emotionally stable, but more conscientious respondents are also significantly more likely to experience mental ill-health compared with more emotionally stable and more conscientious individuals. However, starting at the age of 26, they are (borderline) significantly less likely to have mental health issues compared with less 
emotionally stable and less conscientious individuals.

This result might be related to positive success experiences that are more likely if the respondent overcomes the tendency to avoid challenges, but acts more problemfocused based on higher conscientiousness. Accordingly, the individual's own perceived and actual problem-solving ability might be improved, whereby sustained stress and mental ill-health holds lesser concern. This argumentation is supported by Campbell-Sills et al. (2006) who argue that "the tendency to respond to stressful situations with an active, problem-solving approach promotes resilience in conscientious individuals" (p. 585). There are no significant gender differences regarding both tables.

Table 5.5: Role of Emotional Stability and Conscientiousness - Controlling for Mental Ill-Health at Age 16

\begin{tabular}{lllll}
\hline Mental Ill-Health Score $\ldots$ & Age 26 & Age 30 & Age 34 & Age 42 \\
\hline Emotional Stability (Age 16) & $-0.122^{* * *}$ & $-0.080^{* * *}$ & -0.050 & $-0.126^{* * *}$ \\
& $(0.000)$ & $(0.004)$ & $(0.105)$ & $(0.000)$ \\
Conscientiousness (Age 16) & $-0.050^{*}$ & $-0.046^{*}$ & $-0.096^{* * *}$ & -0.013 \\
& $(0.083)$ & $(0.079)$ & $(0.001)$ & $(0.646)$ \\
Malaise Score (Age 16) & $0.113^{* * *}$ & $0.103^{* * *}$ & $0.097^{* * *}$ & $0.078^{* * *}$ \\
& $(0.000)$ & $(0.000)$ & $(0.000)$ & $(0.000)$ \\
Male & $-0.289^{* * *}$ & $-0.178^{* * *}$ & $-0.186^{* * *}$ & $-0.204^{* * *}$ \\
& $(0.000)$ & $(0.000)$ & $(0.000)$ & $(0.000)$ \\
\hline$N$ & 1907 & 2149 & 1983 & 1797 \\
$R^{2}$ & 0.207 & 0.154 & 0.152 & 0.135 \\
\hline
\end{tabular}

Source: British Cohort Study (BCS70), own calculations.

Note: $p$-values in parentheses $* p<0.10,{ }^{* *} p<0.05,{ }^{* * *} p<0.01$. The OLS regressions include controls for mother's age, mother's age squared, region of living and parental socioeconomic background at birth, the personality traits agreeableness and extraversion measured at the age of 16 as well as cognitive ability measured at the age of 10 .

Yet, one could argue that mental health problems at both adolescent and adult age are strongly related to a genetic predisposition and not behavioral differences. Hence, emotional stability at the age of 16 would only reflect mental ill-health at the age of 16 in the regressions for the older ages. To test for this, the regressions in Tables 5.2 and 5.4 are repeated for the ages of 26, 30, 34 and 42 by additionally controlling for mental health at the age of 16 . Tables 5.5 and 5.6 show that a one-point increase of the malaise score at the age of 16 is on average associated with a mental 
ill-health increase of around 0.1 of a standard deviation at older ages. Nevertheless, the coefficients for emotional stability and the personality type of less emotionally stable and less conscientious individuals remain robust. This supports the argumentation that beside an underlying genetical component, behavioral variation related to personality traits matter for mental health outcomes throughout life (Kendler and Myers, 2010).

Table 5.6: Personality Types and Mental Ill-Health - Controlling for Mental IllHealth at Age 16

\begin{tabular}{lllll}
\hline Mental Ill-Health Score ... & Age 26 & Age 30 & Age 34 & Age 42 \\
\hline High Emotional Stability, & 0.049 & 0.082 & 0.068 & -0.013 \\
Low Conscientiousness (Age 16) & $(0.489)$ & $(0.203)$ & $(0.293)$ & $(0.850)$ \\
Low Emotional Stability, & 0.046 & 0.037 & 0.002 & $0.119^{*}$ \\
High Conscientiousness (Age 16) & $(0.434)$ & $(0.522)$ & $(0.969)$ & $(0.054)$ \\
Low Emotional Stability, & $0.262^{* * *}$ & $0.230^{* * *}$ & $0.232^{* * *}$ & $0.281^{* * *}$ \\
Low Conscientiousness (Age 16) & $(0.001)$ & $(0.001)$ & $(0.001)$ & $(0.000)$ \\
Malaise Score (Age 16) & $0.116^{* * *}$ & $0.104^{* * *}$ & $0.100^{* * *}$ & $0.080^{* * *}$ \\
& $(0.000)$ & $(0.000)$ & $(0.000)$ & $(0.000)$ \\
Male & $-0.298^{* * *}$ & $-0.185^{* * * *}$ & $-0.185^{* * *}$ & $-0.211^{* * *}$ \\
& $(0.000)$ & $(0.000)$ & $(0.000)$ & $(0.000)$ \\
\hline$N$ & 1907 & 2149 & 1983 & 1797 \\
$R^{2}$ & 0.202 & 0.153 & 0.149 & 0.134 \\
\hline
\end{tabular}

Source: British Cohort Study (BCS70), own calculations.

Note: $p$-values in parentheses ${ }^{*} p<0.10,{ }^{* *} p<0.05,{ }^{* * *} p<0.01$. The reference group for the personality types comprises emotionally stable and highly conscientious individuals. The OLS regressions include controls for mother's age, mother's age squared, region of living and parental socioeconomic background at birth, the personality traits agreeableness and extraversion measured at the age of 16 as well as cognitive ability measured at the age of 10 .

\subsubsection{Personality Change and Mental Ill-Health}

To test for recursive causality between personality and mental ill-health at the age of 16 and to analyze the relevance of personality traits at younger age for mental health outcomes at adolescence and adulthood, the relation between personality at the age of 10 as well as a personality trait change between the ages of 10 and 16 and the incidence of mental health problems in adolescence and adulthood is tested. A personality trait increase (decrease) is considered if the particular trait increases 
(decreases) by 0.5 of a standard deviation between the ages of 10 and 16. Individuals who do not experience a personality trait change above or below 0.5 of a standard deviation between the ages of 10 and 16 are considered as the reference group.

Table 5.7 shows that emotional stability at the age of 10 is significantly negatively related to mental ill-health at adolescence and older age. A 1 standard deviation higher level of emotional stability at the age of 10 is associated with a decrease in mental health problems in adolescence or adult age by between 0.08 and 0.12 of a standard deviation. Furthermore, an increase in emotional stability by more than 0.5 of a standard deviation between the ages of 10 and 16 is related to a decrease in mental ill-health at young and older age by between 0.09 and 0.15 of a standard deviation, while a decrease in emotional stability by more than 0.5 of a standard deviation between age 10 and 16 is related to a increase in mental ill-health at young and older age by between 0.09 and 0.25 of a standard deviation.

Table 5.7: Personality Trait Change and Mental Ill-Health

\begin{tabular}{|c|c|c|c|c|c|}
\hline Mental Ill-Health Score ... & Age 16 & Age 26 & Age 30 & Age 34 & Age 42 \\
\hline Emotional Stability (Age 10) & $\begin{array}{l}-0.114^{* * *} \\
(0.000)\end{array}$ & $\begin{array}{l}-0.124^{* * *} \\
(0.000)\end{array}$ & $\begin{array}{l}-0.082^{* * *} \\
(0.001)\end{array}$ & $\begin{array}{l}-0.101^{* * *} \\
(0.000)\end{array}$ & $\begin{array}{l}-0.088^{* * * *} \\
(0.002)\end{array}$ \\
\hline Conscientiousness (Age 10) & $\begin{array}{l}0.006 \\
(0.839)\end{array}$ & $\begin{array}{l}-0.030 \\
(0.246)\end{array}$ & $\begin{array}{l}-0.037 \\
(0.117)\end{array}$ & $\begin{array}{l}-0.073^{* * *} \\
(0.004)\end{array}$ & $\begin{array}{l}-0.058^{* *} \\
(0.025)\end{array}$ \\
\hline $\begin{array}{l}\text { Emotional Stability } \\
(\text { Age } 16-10)>0.5\end{array}$ & $\begin{array}{l}-0.151^{* * *} \\
(0.007)\end{array}$ & $\begin{array}{l}-0.153^{* * *} \\
(0.002)\end{array}$ & $\begin{array}{l}-0.095^{* *} \\
(0.028)\end{array}$ & $\begin{array}{l}-0.141^{* * *} \\
(0.002)\end{array}$ & $\begin{array}{l}-0.088^{*} \\
(0.087)\end{array}$ \\
\hline $\begin{array}{l}\text { Emotional Stability } \\
\text { (Age 16-10) }<-0.5\end{array}$ & $\begin{array}{l}0.254^{* * * *} \\
(0.000)\end{array}$ & $\begin{array}{l}0.085^{* *} \\
(0.050)\end{array}$ & $\begin{array}{l}0.114^{* * *} \\
(0.005)\end{array}$ & $\begin{array}{l}0.140^{* * *} \\
(0.001)\end{array}$ & $\begin{array}{l}0.201^{* * *} \\
(0.000)\end{array}$ \\
\hline $\begin{array}{l}\text { Conscientiousness } \\
(\text { Age 16-10) }>0.5\end{array}$ & $\begin{array}{l}0.054 \\
(0.355)\end{array}$ & $\begin{array}{l}0.015 \\
(0.773)\end{array}$ & $\begin{array}{l}-0.010 \\
(0.816)\end{array}$ & $\begin{array}{l}-0.036 \\
(0.470)\end{array}$ & $\begin{array}{l}-0.075 \\
(0.157)\end{array}$ \\
\hline $\begin{array}{l}\text { Conscientiousness } \\
\text { (Age 16-10) }<-0.5\end{array}$ & $\begin{array}{l}0.112^{* *} \\
(0.042)\end{array}$ & $\begin{array}{l}0.116^{* * *} \\
(0.010)\end{array}$ & $\begin{array}{l}0.118^{* * *} \\
(0.005)\end{array}$ & $\begin{array}{l}0.094^{* *} \\
(0.031)\end{array}$ & $\begin{array}{l}0.033 \\
(0.470)\end{array}$ \\
\hline Male & $\begin{array}{l}-0.329^{* * *} \\
(0.000)\end{array}$ & $\begin{array}{l}-0.361^{* * *} \\
(0.000)\end{array}$ & $\begin{array}{l}-0.265^{* * *} \\
(0.000)\end{array}$ & $\begin{array}{l}-0.240^{* * *} \\
(0.000)\end{array}$ & $\begin{array}{l}-0.209^{* * *} \\
(0.000)\end{array}$ \\
\hline$N$ & 2559 & 3436 & 4200 & 3789 & 3369 \\
\hline$R^{2}$ & 0.106 & 0.097 & 0.069 & 0.068 & 0.066 \\
\hline
\end{tabular}

Source: British Cohort Study (BCS70), own calculations.

Note: $p$-values in parentheses ${ }^{*} p<0.10,{ }^{* *} p<0.05,{ }^{* * *} p<0.01$. The OLS regressions include controls for mother's age, mother's age squared, region of living and parental socioeconomic background at birth, the personality traits agreeableness and extraversion measured at the ages of 10 and 16 as well as cognitive ability measured at the age of 10 . 
Table 5.7 further shows that conscientiousness at the age of 10 is (borderline) negatively related to mental health issues at the ages of 30, 34 and 42. In addition, a decrease in conscientiousness between the ages of 10 and 16 is significantly associated with mental health problems in adolescence and young adulthood. Finally, there is only very limited evidence of significant gender differences in these associations. Detailed results are available in the Appendix Tables 5.16, 5.17 and 5.18 that provide the regressions including gender dummies.

\subsection{Conclusion}

Is the relationship between emotional stability, conscientiousness and the subjective measure mental health in line with the theoretical considerations or does it only hold regarding the objective outcome educational achievement? This questions is addressed by analyzing the predictive power of (1) low emotional stability at the age of 16 for mental health problems at the ages of 16, 26, 30, 34 and 42 as well as the possible substitution effect of conscientiousness and (2) a change in emotional stability and conscientiousness between the ages of 10 and 16 and mental ill-health in later age.

The main results are summarized as follows: First, the study finds that emotional stability at the age of 16 significantly negatively predicts mental health problems in adolescence and adulthood. Less emotionally stable and less conscientious adolescents have the highest risk of experiencing mental health problems throughout life. Higher levels of conscientiousness at the age of 16 significantly mitigate the positive relation at adult age.

Second, emotional stability at the age of 10 significantly predicts mental ill-health at the age of 16 and older. Individuals who increase in emotional stability between the ages of 10 and 16 are significantly less likely to experience mental health problems at the age of 16 and older, while a decrease in emotional stability is associated with an increased risk of mental ill-health.

The results are in line with the theoretical considerations and emphasize the importance of personality development in puberty for later life mental health outcomes (see also Chapter 3). Low emotional stability might be related to a perceived and actual reduced problem-solving ability in challenging situations related to pessimistic expectations and disengagement coping strategies. This is likely to result in sustained 
stress and mental health problems, which is found in the study.

Higher conscientiousness is expected to be associated with an improved individual problem-solving ability related to a boost in control, a reduction in arousal and a higher probability of making positive experiences. This is assumed to be related to better mental health outcomes and found in the empirical analysis.

This idea is further tested in the next chapter. Thereby, it is assumed that pessimistic expectations of helplessness and hopelessness partially mediate the relation between emotional stability, conscientiousness and mental health outcomes. 


\subsection{Appendix}

\subsubsection{Data Description}

Table 5.8: Descriptive Statistics (Age 16)

\begin{tabular}{|c|c|c|c|c|c|c|c|c|c|c|}
\hline \multirow[b]{2}{*}{ Variable Names } & \multicolumn{5}{|c|}{ Working Sample } & \multicolumn{5}{|c|}{ Unrestricted Sample } \\
\hline & $\mathrm{N}$ & Mean & $\mathrm{SD}$ & Min & $\operatorname{Max}$ & $\mathrm{N}$ & Mean & $\mathrm{SD}$ & Min & $\operatorname{Max}$ \\
\hline Malaise Score (Age 16) & 2559 & -0.03 & 0.98 & -1.51 & 4.15 & 4673 & 0.00 & 1.00 & -1.51 & 4.86 \\
\hline Emotional Stability (ES, Age 10) & 2559 & 0.12 & 0.90 & -3.23 & 1.26 & 11796 & 0.00 & 1.00 & -3.51 & 1.34 \\
\hline Conscientiousness (C, Age 10) & 2559 & 0.19 & 0.87 & -3.31 & 1.20 & 11796 & 0.00 & 1.00 & -3.35 & 1.20 \\
\hline Agreeableness (A, Age 10) & 2559 & 0.14 & 0.79 & -5.79 & 1.27 & 11796 & 0.00 & 1.00 & -6.48 & 1.38 \\
\hline Extraversion (E, Age 10) & 2559 & 0.01 & 1.00 & -3.27 & 1.47 & 11796 & -0.00 & 1.00 & -3.62 & 1.47 \\
\hline Emotional Stability (ES, Age 16) & 2559 & 0.08 & 0.92 & -4.00 & 0.85 & 7132 & -0.00 & 1.00 & -4.00 & 0.85 \\
\hline Conscientiousness (C, Age 16) & 2559 & 0.14 & 0.88 & -4.65 & 0.72 & 7132 & -0.00 & 1.00 & -4.65 & 0.72 \\
\hline Agreeableness (A, Age 16) & 2559 & 0.17 & 0.77 & -6.54 & 0.53 & 7132 & 0.00 & 1.00 & -6.61 & 0.53 \\
\hline Extraversion (E, Age 16) & 2559 & 0.00 & 0.98 & -4.82 & 0.96 & 7132 & 0.00 & 1.00 & -4.82 & 0.96 \\
\hline High ES, High C (Age 16) & 2559 & 0.55 & 0.50 & 0.00 & 1.00 & 7132 & 0.49 & 0.50 & 0.00 & 1.00 \\
\hline High ES, Low C (Age 16) & 2559 & 0.12 & 0.32 & 0.00 & 1.00 & 7132 & 0.14 & 0.35 & 0.00 & 1.00 \\
\hline Low ES, High C (Age 16) & 2559 & 0.16 & 0.37 & 0.00 & 1.00 & 7132 & 0.16 & 0.37 & 0.00 & 1.00 \\
\hline Low ES, Low C (Age 16) & 2559 & 0.17 & 0.38 & 0.00 & 1.00 & 7132 & 0.21 & 0.41 & 0.00 & 1.00 \\
\hline Male & 2559 & 0.41 & 0.49 & 0.00 & 1.00 & 17185 & 0.52 & 0.50 & 0.00 & 1.00 \\
\hline Cognitive Ability (Age 10) & 2559 & 0.39 & 0.88 & -3.21 & 2.44 & 11598 & -0.00 & 1.00 & -4.42 & 2.49 \\
\hline Higher SES Parents (Birth) & 2559 & 0.23 & 0.42 & 0.00 & 1.00 & 17164 & 0.18 & 0.39 & 0.00 & 1.00 \\
\hline Region (Birth) & 2559 & 5.71 & 2.70 & 1.00 & 10.00 & 17185 & 5.89 & 2.70 & 1.00 & 11.00 \\
\hline Age Mother & 2559 & 23.02 & 3.98 & 14.00 & 42.00 & 17067 & 22.15 & 4.03 & 12.00 & 47.00 \\
\hline
\end{tabular}

Source: BCS70, own calculations. SES refers to the term socioeconomic status.

Table 5.9: Descriptive Statistics (Age 26)

\begin{tabular}{|c|c|c|c|c|c|c|c|c|c|c|}
\hline \multirow[b]{2}{*}{ Variable Names } & \multicolumn{3}{|c|}{ Working Sample } & \multirow[b]{2}{*}{ Min } & \multirow[b]{2}{*}{$\operatorname{Max}$} & \multicolumn{3}{|c|}{ Unrestricted Sample } & \multirow[b]{2}{*}{ Min } & \multirow[b]{2}{*}{$\operatorname{Max}$} \\
\hline & $\mathrm{N}$ & Mean & SD & & & $\mathrm{N}$ & Mean & SD & & \\
\hline Malaise Score (Age 26) & 3436 & -0.05 & 0.96 & -1.00 & 4.07 & 8044 & -0.00 & 1.00 & -1.00 & 4.07 \\
\hline Emotional Stability (ES, Age 10) & 3436 & 0.13 & 0.89 & -3.37 & 1.26 & 11796 & 0.00 & 1.00 & -3.51 & 1.34 \\
\hline Conscientiousness (C, Age 10) & 3436 & 0.16 & 0.89 & -3.11 & 1.20 & 11796 & 0.00 & 1.00 & -3.35 & 1.20 \\
\hline Agreeableness (A, Age 10) & 3436 & 0.12 & 0.82 & -5.98 & 1.27 & 11796 & 0.00 & 1.00 & -6.48 & 1.38 \\
\hline Extraversion (E, Age 10) & 3436 & 0.01 & 0.98 & -3.55 & 1.47 & 11796 & -0.00 & 1.00 & -3.62 & 1.47 \\
\hline Emotional Stability (ES, Age 16) & 3436 & 0.08 & 0.94 & -4.00 & 0.85 & 7132 & -0.00 & 1.00 & -4.00 & 0.85 \\
\hline Conscientiousness (C, Age 16) & 3436 & 0.12 & 0.89 & -4.65 & 0.72 & 7132 & -0.00 & 1.00 & -4.65 & 0.72 \\
\hline Agreeableness (A, Age 16) & 3436 & 0.11 & 0.89 & -6.54 & 0.53 & 7132 & 0.00 & 1.00 & -6.61 & 0.53 \\
\hline Extraversion (E, Age 16) & 3436 & 0.02 & 0.97 & -4.82 & 0.96 & 7132 & 0.00 & 1.00 & -4.82 & 0.96 \\
\hline High ES, High C (Age 16) & 3436 & 0.54 & 0.50 & 0.00 & 1.00 & 7132 & 0.49 & 0.50 & 0.00 & 1.00 \\
\hline High ES, Low C (Age 16) & 3436 & 0.13 & 0.34 & 0.00 & 1.00 & 7132 & 0.14 & 0.35 & 0.00 & 1.00 \\
\hline
\end{tabular}




\begin{tabular}{|c|c|c|c|c|c|c|c|c|c|c|}
\hline \multirow[b]{2}{*}{ Variable Names } & \multicolumn{3}{|c|}{ Working Sample } & \multirow[b]{2}{*}{ Min } & \multirow[b]{2}{*}{ Max } & \multicolumn{4}{|c|}{ Unrestricted Sample } & \multirow[b]{2}{*}{$\operatorname{Max}$} \\
\hline & $\mathrm{N}$ & Mean & SD & & & $\mathrm{N}$ & Mean & SD & Min & \\
\hline Low ES, High C (Age 16) & 3436 & 0.16 & 0.37 & 0.00 & 1.00 & 7132 & 0.16 & 0.37 & 0.00 & 1.00 \\
\hline Low ES, Low C (Age 16) & 3436 & 0.17 & 0.38 & 0.00 & 1.00 & 7132 & 0.21 & 0.41 & 0.00 & 1.00 \\
\hline Male & 3436 & 0.43 & 0.49 & 0.00 & 1.00 & 17185 & 0.52 & 0.50 & 0.00 & 1.00 \\
\hline Cognitive Ability (Age 10) & 3436 & 0.32 & 0.88 & -3.21 & 2.44 & 11598 & -0.00 & 1.00 & -4.42 & 2.49 \\
\hline Higher SES Parents (Birth) & 3436 & 0.21 & 0.41 & 0.00 & 1.00 & 17164 & 0.18 & 0.39 & 0.00 & 1.00 \\
\hline Region (Birth) & 3436 & 5.61 & 2.69 & 1.00 & 10.00 & 17185 & 5.89 & 2.70 & 1.00 & 11.00 \\
\hline Age Mother & 3436 & 22.85 & 3.96 & 15.00 & 46.00 & 17067 & 22.15 & 4.03 & 12.00 & 47.00 \\
\hline
\end{tabular}

Source: BCS70, own calculations. SES refers to the term socioeconomic status.

Table 5.10: Descriptive Statistics (Age 30)

\begin{tabular}{|c|c|c|c|c|c|c|c|c|c|c|}
\hline \multirow[b]{2}{*}{ Variable Names } & \multicolumn{3}{|c|}{ Working Sample } & \multirow[b]{2}{*}{ Min } & \multirow[b]{2}{*}{ Max } & \multicolumn{4}{|c|}{ Unrestricted Sample } & \multirow[b]{2}{*}{$\operatorname{Max}$} \\
\hline & $\mathrm{N}$ & Mean & SD & & & $\mathrm{N}$ & Mean & SD & Min & \\
\hline Malaise Score (Age 30) & 4200 & -0.05 & 0.96 & -0.88 & 4.24 & 10251 & -0.00 & 1.00 & -0.88 & 4.24 \\
\hline Emotional Stability (ES, Age 10) & 4200 & 0.10 & 0.92 & -3.45 & 1.28 & 11796 & 0.00 & 1.00 & -3.51 & 1.34 \\
\hline Conscientiousness (C, Age 10) & 4200 & 0.11 & 0.92 & -3.31 & 1.20 & 11796 & 0.00 & 1.00 & -3.35 & 1.20 \\
\hline Agreeableness (A, Age 10) & 4200 & 0.08 & 0.87 & -6.37 & 1.27 & 11796 & 0.00 & 1.00 & -6.48 & 1.38 \\
\hline Extraversion (E, Age 10) & 4200 & 0.01 & 0.98 & -3.61 & 1.47 & 11796 & -0.00 & 1.00 & -3.62 & 1.47 \\
\hline Emotional Stability (ES, Age 16) & 4200 & 0.06 & 0.95 & -4.00 & 0.85 & 7132 & -0.00 & 1.00 & -4.00 & 0.85 \\
\hline Conscientiousness (C, Age 16) & 4200 & 0.06 & 0.93 & -4.65 & 0.72 & 7132 & -0.00 & 1.00 & -4.65 & 0.72 \\
\hline Agreeableness (A, Age 16) & 4200 & 0.06 & 0.93 & -6.61 & 0.53 & 7132 & 0.00 & 1.00 & -6.61 & 0.53 \\
\hline Extraversion (E, Age 16) & 4200 & 0.03 & 0.96 & -4.82 & 0.96 & 7132 & 0.00 & 1.00 & -4.82 & 0.96 \\
\hline High ES, High C (Age 16) & 4200 & 0.51 & 0.50 & 0.00 & 1.00 & 7132 & 0.49 & 0.50 & 0.00 & 1.00 \\
\hline High ES, Low C (Age 16) & 4200 & 0.14 & 0.35 & 0.00 & 1.00 & 7132 & 0.14 & 0.35 & 0.00 & 1.00 \\
\hline Low ES, High C (Age 16) & 4200 & 0.16 & 0.36 & 0.00 & 1.00 & 7132 & 0.16 & 0.37 & 0.00 & 1.00 \\
\hline Low ES, Low C (Age 16) & 4200 & 0.19 & 0.39 & 0.00 & 1.00 & 7132 & 0.21 & 0.41 & 0.00 & 1.00 \\
\hline Male & 4200 & 0.46 & 0.50 & 0.00 & 1.00 & 17185 & 0.52 & 0.50 & 0.00 & 1.00 \\
\hline Cognitive Ability (Age 10) & 4200 & 0.23 & 0.92 & -3.21 & 2.44 & 11598 & -0.00 & 1.00 & -4.42 & 2.49 \\
\hline Higher SES Parents (Birth) & 4200 & 0.20 & 0.40 & 0.00 & 1.00 & 17164 & 0.18 & 0.39 & 0.00 & 1.00 \\
\hline Region (Birth) & 4200 & 5.66 & 2.70 & 1.00 & 10.00 & 17185 & 5.89 & 2.70 & 1.00 & 11.00 \\
\hline Age Mother & 4200 & 22.65 & 3.95 & 14.00 & 46.00 & 17067 & 22.15 & 4.03 & 12.00 & 47.00 \\
\hline
\end{tabular}

Source: BCS70, own calculations. SES refers to the term socioeconomic status.

Table 5.11: Descriptive Statistics (Age 34)

\begin{tabular}{|c|c|c|c|c|c|c|c|c|c|c|}
\hline \multirow[b]{2}{*}{ Variable Names } & \multicolumn{3}{|c|}{ Working Sample } & \multirow[b]{2}{*}{ Min } & \multirow[b]{2}{*}{ Max } & \multicolumn{4}{|c|}{ Unrestricted Sample } & \multirow[b]{2}{*}{$\operatorname{Max}$} \\
\hline & $\mathrm{N}$ & Mean & SD & & & $\mathrm{N}$ & Mean & SD & Min & \\
\hline Malaise Score (Age 34) & 3789 & -0.04 & 0.98 & -0.88 & 3.87 & 8866 & 0.00 & 1.00 & -0.88 & 3.87 \\
\hline Emotional Stability (ES, Age 10) & 3789 & 0.11 & 0.91 & -3.45 & 1.26 & 11796 & 0.00 & 1.00 & -3.51 & 1.34 \\
\hline Conscientiousness (C, Age 10) & 3789 & 0.12 & 0.92 & -3.31 & 1.20 & 11796 & 0.00 & 1.00 & -3.35 & 1.20 \\
\hline Agreeableness (A, Age 10) & 3789 & 0.10 & 0.84 & -6.37 & 1.26 & 11796 & 0.00 & 1.00 & -6.48 & 1.38 \\
\hline Extraversion (E, Age 10) & 3789 & 0.02 & 0.99 & -3.61 & 1.47 & 11796 & -0.00 & 1.00 & -3.62 & 1.47 \\
\hline Emotional Stability (ES, Age 16) & 3789 & 0.07 & 0.94 & -4.00 & 0.85 & 7132 & -0.00 & 1.00 & -4.00 & 0.85 \\
\hline
\end{tabular}




\begin{tabular}{|c|c|c|c|c|c|c|c|c|c|c|}
\hline \multirow[b]{2}{*}{ Variable Names } & \multicolumn{3}{|c|}{ Working Sample } & \multirow[b]{2}{*}{ Min } & \multirow[b]{2}{*}{ Max } & \multicolumn{3}{|c|}{ Unrestricted Sample } & \multirow[b]{2}{*}{ Min } & \multirow[b]{2}{*}{ Max } \\
\hline & & Mean & SD & & & $\mathrm{N}$ & Mean & $\mathrm{SD}$ & & \\
\hline Conscientiousness (C, Age 16) & 3789 & 0.08 & 0.92 & -4.65 & 0.72 & 7132 & -0.00 & 1.00 & -4.65 & 0.72 \\
\hline Agreeableness (A, Age 16) & 3789 & 0.08 & 0.90 & -6.54 & 0.53 & 7132 & 0.00 & 1.00 & -6.61 & 0.53 \\
\hline Extraversion (E, Age 16) & 3789 & 0.03 & 0.97 & -4.82 & 0.96 & 7132 & 0.00 & 1.00 & -4.82 & 0.96 \\
\hline High ES, High C (Age 16) & 3789 & 0.52 & 0.50 & 0.00 & 1.00 & 7132 & 0.49 & 0.50 & 0.00 & 1.00 \\
\hline High ES, Low C (Age 16) & 3789 & 0.14 & 0.35 & 0.00 & 1.00 & 7132 & 0.14 & 0.35 & 0.00 & 1.00 \\
\hline Low ES, High C (Age 16) & 3789 & 0.16 & 0.36 & 0.00 & 1.00 & 7132 & 0.16 & 0.37 & 0.00 & 1.00 \\
\hline Low ES, Low C (Age 16) & 3789 & 0.18 & 0.39 & 0.00 & 1.00 & 7132 & 0.21 & 0.41 & 0.00 & 1.00 \\
\hline Male & 3789 & 0.45 & 0.50 & 0.00 & 1.00 & 17185 & 0.52 & 0.50 & 0.00 & 1.00 \\
\hline Cognitive Ability (Age 10) & 3789 & 0.26 & 0.91 & -3.53 & 2.44 & 11598 & -0.00 & 1.00 & -4.42 & 2.49 \\
\hline Higher SES Parents (Birth) & 3789 & 0.21 & 0.41 & 0.00 & 1.00 & 17164 & 0.18 & 0.39 & 0.00 & 1.00 \\
\hline Region (Birth) & 3789 & 5.65 & 2.69 & 1.00 & 10.00 & 17185 & 5.89 & 2.70 & 1.00 & 11.00 \\
\hline Age Mother (0) & 3789 & 22.76 & 3.93 & 14.00 & 46.00 & 17067 & 22.15 & 4.03 & 12.00 & 47.00 \\
\hline
\end{tabular}

Source: BCS70, own calculations. SES refers to the term socioeconomic status.

Table 5.12: Descriptive Statistics (Age 42)

\begin{tabular}{|c|c|c|c|c|c|c|c|c|c|c|}
\hline \multirow[b]{2}{*}{ Variable Names } & \multicolumn{3}{|c|}{ Working Sample } & \multirow[b]{2}{*}{ Min } & \multirow[b]{2}{*}{$\operatorname{Max}$} & \multicolumn{4}{|c|}{ Unrestricted Sample } & \multirow[b]{2}{*}{$\operatorname{Max}$} \\
\hline & $\mathrm{N}$ & Mean & SD & & & $\mathrm{N}$ & Mean & SD & Min & \\
\hline Malaise Score (Age 42) & 3369 & -0.04 & 0.96 & -0.93 & 3.57 & 7935 & 0.00 & 1.00 & -0.93 & 3.57 \\
\hline Emotional Stability (ES, Age 10) & 3369 & 0.11 & 0.91 & -3.45 & 1.26 & 11796 & 0.00 & 1.00 & -3.51 & 1.34 \\
\hline Conscientiousness (C, Age 10) & 3369 & 0.14 & 0.91 & -3.31 & 1.20 & 11796 & 0.00 & 1.00 & -3.35 & 1.20 \\
\hline Agreeableness (A, Age 10) & 3369 & 0.12 & 0.83 & -6.37 & 1.26 & 11796 & 0.00 & 1.00 & -6.48 & 1.38 \\
\hline Extraversion (E, Age 10) & 3369 & 0.02 & 0.99 & -3.61 & 1.47 & 11796 & -0.00 & 1.00 & -3.62 & 1.47 \\
\hline Emotional Stability (ES, Age 16) & 3369 & 0.06 & 0.95 & -4.00 & 0.85 & 7132 & -0.00 & 1.00 & -4.00 & 0.85 \\
\hline Conscientiousness (C, Age 16) & 3369 & 0.10 & 0.90 & -4.65 & 0.72 & 7132 & -0.00 & 1.00 & -4.65 & 0.72 \\
\hline Agreeableness (A, Age 16) & 3369 & 0.09 & 0.90 & -6.54 & 0.53 & 7132 & 0.00 & 1.00 & -6.61 & 0.53 \\
\hline Extraversion (E, Age 16) & 3369 & 0.02 & 0.96 & -4.82 & 0.96 & 7132 & 0.00 & 1.00 & -4.82 & 0.96 \\
\hline High ES, High C (Age 16) & 3369 & 0.53 & 0.50 & 0.00 & 1.00 & 7132 & 0.49 & 0.50 & 0.00 & 1.00 \\
\hline High ES, Low C (Age 16) & 3369 & 0.13 & 0.34 & 0.00 & 1.00 & 7132 & 0.14 & 0.35 & 0.00 & 1.00 \\
\hline Low ES, High C (Age 16) & 3369 & 0.16 & 0.37 & 0.00 & 1.00 & 7132 & 0.16 & 0.37 & 0.00 & 1.00 \\
\hline Low ES, Low C (Age 16) & 3369 & 0.18 & 0.38 & 0.00 & 1.00 & 7132 & 0.21 & 0.41 & 0.00 & 1.00 \\
\hline Male & 3369 & 0.44 & 0.50 & 0.00 & 1.00 & 17185 & 0.52 & 0.50 & 0.00 & 1.00 \\
\hline Cognitive Ability (Age 10) & 3369 & 0.28 & 0.90 & -3.74 & 2.44 & 11598 & -0.00 & 1.00 & -4.42 & 2.49 \\
\hline Higher SES Parents (Birth) & 3369 & 0.22 & 0.41 & 0.00 & 1.00 & 17164 & 0.18 & 0.39 & 0.00 & 1.00 \\
\hline Region (Birth) & 3369 & 5.54 & 2.66 & 1.00 & 10.00 & 17185 & 5.89 & 2.70 & 1.00 & 11.00 \\
\hline Age Mother & 3369 & 22.77 & 3.94 & 14.00 & 46.00 & 17067 & 22.15 & 4.03 & 12.00 & 47.00 \\
\hline
\end{tabular}

Source: BCS70, own calculations. SES refers to the term socioeconomic status. 
Table 5.13: Malaise Items

\begin{tabular}{lllllll}
\hline Question & Age 16 & Age 26 & Age 30 & Age 34 & Age 38 & Age 42 \\
\hline 1. Do you often have backache? & $\sqrt{ }$ & $\sqrt{ }$ & $\sqrt{ }$ & $\otimes$ & $\otimes$ & $\otimes$
\end{tabular}

2. Do you feel tired most of the time?

3. Do you often feel depressed?

4. Do you often have bad

headaches?

5. Do you often get worried about things?

6. Do you usually have great difficulty in falling or staying asleep?

7. Do you usually wake unnecessarily early in the morning?

8. Do you wear yourself out worrying about your health?

9. Do you often get into violent rage?

10. Do people annoy and irritate you?

11. Have you at times had a twitching of the face, head or shoulders?

12. Do you suddenly become scared for no good reason?

13. Are you scared to be alone when there are no friends near you?

14. Are you easily upset or irritated?

15. Are you frightened of going out alone or of meeting people?

16. Are constantly keyed up and jittery?

17. Do you suffer from indigestion?

18. Do you suffer from an upset stomach?

19. Is your appetite poor?

20. Does every little thing get on your nerves and wear you out? 
... Table 5.13 continued

\begin{tabular}{|c|c|c|c|c|c|c|}
\hline Question & Age 16 & Age 26 & Age 30 & Age 34 & Age 38 & Age 42 \\
\hline $\begin{array}{l}\text { 21. Does your heart often race } \\
\text { like mad? }\end{array}$ & $\sqrt{ }$ & $\sqrt{ }$ & $\sqrt{ }$ & $\sqrt{ }$ & $\otimes$ & $\sqrt{ }$ \\
\hline $\begin{array}{l}\text { 22. Do you often have bad pain in } \\
\text { eyes? }\end{array}$ & $\sqrt{ }$ & $\sqrt{ }$ & $\sqrt{ }$ & $\otimes$ & $\otimes$ & $\otimes$ \\
\hline $\begin{array}{l}23 . \text { Are you troubled with rheuma- } \\
\text { tism or fibrosis? }\end{array}$ & $\otimes$ & $\sqrt{ }$ & $\sqrt{ }$ & $\otimes$ & $\otimes$ & $\otimes$ \\
\hline $\begin{array}{l}24 . \text { Have you ever had a nervous } \\
\text { breakdown? }\end{array}$ & $\otimes$ & $\sqrt{ }$ & $\sqrt{ }$ & $\otimes$ & $\otimes$ & $\otimes$ \\
\hline Number of malaise items & 22 & 24 & 24 & 9 & 0 & 9 \\
\hline $\begin{array}{l}\text { Measurement scale } \\
\text { per malaise item }\end{array}$ & 3 -point & 2-point & 2-point & 2-point & - & 2-point \\
\hline $\begin{array}{l}\text { Measurement scale } \\
\text { per malaise score }\end{array}$ & $0-44$ & $0-24$ & $0-24$ & $0-9$ & - & $0-9$ \\
\hline Threshold for depression & 15 & 8 & 8 & 4 & - & 4 \\
\hline $\begin{array}{l}\text { Unrestricted number } \\
\text { of valid observations }\end{array}$ & 4,673 & 8,044 & 10,251 & 8,866 & 0 & 7,935 \\
\hline
\end{tabular}

Source: BCS70, own calculations.

Note: The nine items that are marked bold are used in the analysis. 
Table 5.14: Personality Traits at the Ages of 10 and 16

\begin{tabular}{|c|c|c|c|c|}
\hline $\begin{array}{l}\text { Personality } \\
\text { Trait }\end{array}$ & $\begin{array}{l}\text { Correlation } \\
80-86\end{array}$ & $\begin{array}{l}\text { Explained } \\
\text { Variance }\end{array}$ & $\begin{array}{l}\text { Cronbach's } \\
\text { Alpha }\end{array}$ & Items \\
\hline \multicolumn{5}{|c|}{ Emotional Stability } \\
\hline Age 10 & & $53.31 \%$ & 0.82 & R8. Irritable. \\
\hline Age 16 & 0.40 & $55.63 \%$ & 0.84 & $\begin{array}{l}\text { R9. Miserable and tearful. } \\
\text { C8. Requests must be met. } \\
\text { C12. Sullen or sulky. } \\
\text { C16. Changes mood quickly. } \\
\text { C17. Temper unpredictable. }\end{array}$ \\
\hline
\end{tabular}

Conscientiousness

Age 10

$\begin{array}{lll} & 66.34 \% & 0.83 \\ 0.44 & 63.98 \% & 0.81\end{array}$

0.83
0.81

R15. Cannot settle.

Age 16

0.44

$63.98 \%$

C3. Easily distracted.

C13. Fails to finish things.

C19. Difficulty concentrating.

Agreeableness

Age 10

$46.90 \%$

0.81

R3. Destroys belongings.

Age 16

0.31

$38.93 \%$

0.74

R4. Frequently fights.

R10. Takes others' belongings.

R14. Often disobedient.

R18. Often tells lies.

R19. Bullies other children.

C11. Interferes with others.

Extraversion

\begin{tabular}{|c|c|c|c|c|}
\hline \multirow{2}{*}{$\begin{array}{l}\text { Age } 10 \\
\text { Age } 16\end{array}$} & & $38.21 \%$ & 0.58 & R5. Not much liked by others. \\
\hline & 0.37 & $36.89 \%$ & 0.56 & R6. Often worried. \\
\hline & & & & R7. Rather solitary. \\
\hline & & & & $\begin{array}{l}\text { R16. Afraid of new things. } \\
\text { R17. Fussy or over-particular. }\end{array}$ \\
\hline
\end{tabular}

Source: BCS70, own calculations based on working sample $\mathrm{n}=2559$.

Note: Items are stated in original terms, but reversely used in the analysis. 
Table 5.15: Correlation between Personality and Mental Ill-Health

\begin{tabular}{|c|c|c|c|c|c|}
\hline \multirow[b]{2}{*}{ Personality Traits and Items } & \multicolumn{2}{|c|}{ Mental Ill-Health } & \multirow[b]{2}{*}{ Age 30} & \multirow[b]{2}{*}{ Age 34} & \multirow[b]{2}{*}{ Age 42} \\
\hline & Age 16 & Age 26 & & & \\
\hline \multicolumn{6}{|l|}{ Emotional Stability } \\
\hline Age 10 & -.11 & -.13 & -.12 & -.12 & -.12 \\
\hline Age 16 & -.24 & -.20 & -.19 & -.18 & -.19 \\
\hline R8. Irritable & -.18 & -.17 & -.14 & -.15 & -.14 \\
\hline R9. Miserable and tearful. & -.20 & -.17 & -.16 & -.17 & -.19 \\
\hline C8. Requests must be met. & -.12 & -.11 & -.11 & -.10 & -.11 \\
\hline C12. Sullen or sulky. & -.20 & -.13 & -.14 & -.15 & -.15 \\
\hline C16. Changes mood quickly. & -.20 & -.17 & -.15 & -.14 & -.15 \\
\hline C17. Temper unpredictable. & -.18 & -.15 & -.14 & -.13 & -.12 \\
\hline \multicolumn{6}{|l|}{ Conscientiousness } \\
\hline Age 10 & -.04 & -.07 & -.08 & -.10 & -.09 \\
\hline Age 16 & -.12 & -.14 & -.14 & -.16 & -.11 \\
\hline R15. Cannot settle. & -.08 & -.11 & -.08 & -.12 & -.10 \\
\hline C3. Easily distracted. & -.09 & -.11 & -.12 & -.12 & -.08 \\
\hline C13. Fails to finish things. & -.10 & -.11 & -.13 & -.14 & -.09 \\
\hline C19. Difficulty concentrating. & -.09 & -.11 & -.10 & -.13 & -.06 \\
\hline \multicolumn{6}{|l|}{ Agreeableness } \\
\hline Age 10 & -.07 & -.07 & -.09 & -.08 & -.10 \\
\hline Age 16 & -.11 & -.10 & -.09 & -.12 & -.10 \\
\hline R3. Destroys belongings. & -.04 & -.05 & -.05 & -.07 & -.08 \\
\hline R4. Frequently fights. & -.07 & -.06 & -.04 & -.07 & -.08 \\
\hline R10. Takes others' belongings. & -.04 & -.04 & -.02 & -.07 & -.07 \\
\hline R14. Often disobedient. & -.10 & -.12 & -.10 & -.12 & -.10 \\
\hline R18. Often tells lies. & -.08 & -.08 & -.10 & -.08 & -.09 \\
\hline R19. Bullies other children. & -.06 & -.04 & -.02 & -.04 & . \\
\hline C11. Interferes with others. & -.07 & -.07 & -.05 & -.09 & -.04 \\
\hline \multicolumn{6}{|l|}{ Extraversion } \\
\hline Age 10 & -.13 & -.11 & -.12 & -.10 & -.11 \\
\hline Age 16 & -.22 & -.19 & -.16 & -.14 & -.18 \\
\hline R5. Not much liked by others. & -.09 & -.06 & -.07 & -.08 & -.07 \\
\hline R6. Often worried. & -.23 & -.19 & -.16 & -.14 & -.17 \\
\hline R7. Rather solitary. & -.09 & -.10 & -.07 & -.04 & -.07 \\
\hline R16. Afraid of new things. & -.14 & -.12 & -.11 & -.10 & -.13 \\
\hline R17. Fussy or over-particular. & -.08 & -.09 & -.05 & -.06 & -.08 \\
\hline
\end{tabular}

Source: BCS70, own calculations.

Note: Items are stated in original terms, but reversely used in the analysis. Correlation coefficients reported are significant at the $5 \%$ level or higher. Mental health problems at the ages of 16 $(\mathrm{n}=2559), 26(\mathrm{n}=3436), 30(\mathrm{n}=4200), 34(\mathrm{n}=3789)$, and $42(\mathrm{n}=3369)$. 


\subsubsection{Robustness Checks}

\subsubsection{Gender Differences}

Table 5.16: Role of Emotional Stability and Conscientiousness by Gender

\begin{tabular}{llllll}
\hline Mental Ill-Health Score ... & Age 16 & Age 26 & Age 30 & Age 34 & Age 42 \\
\hline Emotional Stability (Age 16) & $-0.175^{* * *}$ & $-0.096^{* * *}$ & $-0.129^{* * *}$ & $-0.080^{* *}$ & $-0.146^{* * *}$ \\
& $(0.000)$ & $(0.001)$ & $(0.000)$ & $(0.012)$ & $(0.000)$ \\
Conscientiousness (Age 16) & -0.009 & $-0.084^{* * *}$ & $-0.073^{* *}$ & $-0.076^{* *}$ & -0.024 \\
& $(0.784)$ & $(0.005)$ & $(0.012)$ & $(0.014)$ & $(0.411)$ \\
Emotional Stability & 0.030 & -0.030 & 0.024 & -0.007 & 0.077 \\
Male (Age 16) & $(0.610)$ & $(0.541)$ & $(0.580)$ & $(0.893)$ & $(0.119)$ \\
Conscientiousness & -0.043 & 0.053 & 0.007 & -0.021 & 0.022 \\
Male (Age 16) & $(0.383)$ & $(0.189)$ & $(0.860)$ & $(0.619)$ & $(0.590)$ \\
Male & $-0.332^{* * *}$ & $-0.345^{* * *}$ & $-0.251^{* * *}$ & $-0.240^{* * *}$ & $-0.194^{* * *}$ \\
& $(0.000)$ & $(0.000)$ & $(0.000)$ & $(0.000)$ & $(0.000)$ \\
\hline$N$ & 2559 & 3436 & 4200 & 3789 & 3369 \\
$R^{2}$ & 0.106 & 0.101 & 0.069 & 0.068 & 0.065 \\
\hline
\end{tabular}

Source: British Cohort Study (BCS70), own calculations.

Note: $p$-values in parentheses ${ }^{*} p<0.10,{ }^{* *} p<0.05,{ }^{* * *} p<0.01$. The OLS regressions include controls for mother's age, mother's age squared, region of living and parental socioeconomic background at birth, the personality traits agreeableness and extraversion measured at the age of 16 as well as cognitive ability measured at the age of 10 . 
Table 5.17: Personality Types and Mental Ill-Health by Gender

\begin{tabular}{llllll}
\hline Mental Ill-Health Score ... & Age 16 & Age 26 & Age 30 & Age 34 & Age 42 \\
\hline High Emotional Stability, & -0.007 & 0.029 & 0.122 & 0.085 & 0.039 \\
Low Conscientiousness (Age 16) & $(0.931)$ & $(0.708)$ & $(0.109)$ & $(0.272)$ & $(0.619)$ \\
Low Emotional Stability, & $0.278^{* * *}$ & $0.099^{*}$ & $0.146^{* *}$ & $0.151^{* *}$ & $0.184^{* * *}$ \\
High Conscientiousness (Age 16) & $(0.000)$ & $(0.093)$ & $(0.010)$ & $(0.011)$ & $(0.002)$ \\
Low Emotional Stability, & $0.348^{* * *}$ & $0.274^{* * *}$ & $0.364^{* * *}$ & $0.346^{* * *}$ & $0.330^{* * *}$ \\
Low Conscientiousness (Age 16) & $(0.000)$ & $(0.000)$ & $(0.000)$ & $(0.000)$ & $(0.000)$ \\
High Emotional Stability, Low & 0.180 & 0.039 & 0.028 & -0.010 & -0.102 \\
Conscientiousness Male (Age 16) & $(0.140)$ & $(0.696)$ & $(0.774)$ & $(0.918)$ & $(0.318)$ \\
Low Emotional Stability, High & -0.079 & -0.094 & -0.088 & -0.144 & -0.067 \\
Conscientiousness Male (Age 16) & $(0.480)$ & $(0.291)$ & $(0.303)$ & $(0.111)$ & $(0.489)$ \\
Low Emotional Stability, Low & -0.104 & -0.027 & -0.056 & -0.081 & $-0.198^{* *}$ \\
Conscientiousness Male (Age 16) & $(0.360)$ & $(0.790)$ & $(0.520)$ & $(0.393)$ & $(0.045)$ \\
Male & $-0.332^{* * *}$ & $-0.341^{* * *}$ & $-0.242^{* * *}$ & $-0.202^{* * *}$ & $-0.136^{* * *}$ \\
\hline$N$ & $(0.000)$ & $(0.000)$ & $(0.000)$ & $(0.000)$ & $(0.002)$ \\
\hline$R^{2}$ & 2559 & 3436 & 4200 & 3789 & 3369 \\
\hline
\end{tabular}

Source: British Cohort Study (BCS70), own calculations.

Note: $p$-values in parentheses $* p<0.10,{ }^{*} p<0.05,{ }^{* * *} p<0.01$. The reference group for the personality types comprises emotionally stable and highly conscientious individuals. The OLS regressions include controls for mother's age, mother's age squared, region of living and parental socioeconomic background at birth, the personality traits agreeableness and extraversion measured at the age of 16 as well as cognitive ability measured at the age of 10 . 
Table 5.18: Personality Trait Change and Mental Ill-Health by Gender

\begin{tabular}{|c|c|c|c|c|c|}
\hline Mental Ill-Health Score ... & Age 16 & Age 26 & Age 30 & Age 34 & Age 42 \\
\hline Emotional Stability (Age 10) & $\begin{array}{l}-0.164^{* * *} \\
(0.000)\end{array}$ & $\begin{array}{l}-0.108^{* * *} \\
(0.008)\end{array}$ & $\begin{array}{l}-0.108^{* * *} \\
(0.003)\end{array}$ & $\begin{array}{l}-0.101^{* * *} \\
(0.005)\end{array}$ & $\begin{array}{l}-0.131^{* * *} \\
(0.000)\end{array}$ \\
\hline Conscientiousness (Age 10) & $\begin{array}{l}0.012 \\
(0.772)\end{array}$ & $\begin{array}{l}-0.076^{*} \\
(0.061)\end{array}$ & $\begin{array}{l}-0.052 \\
(0.174)\end{array}$ & $\begin{array}{l}-0.079^{* *} \\
(0.045)\end{array}$ & $\begin{array}{l}-0.065 \\
(0.101)\end{array}$ \\
\hline Emotional Stability Male (Age 10) & $\begin{array}{l}0.114^{*} \\
(0.057)\end{array}$ & $\begin{array}{l}-0.022 \\
(0.697)\end{array}$ & $\begin{array}{l}0.066 \\
(0.170)\end{array}$ & $\begin{array}{l}0.006 \\
(0.914)\end{array}$ & $\begin{array}{l}0.102^{*} \\
(0.073)\end{array}$ \\
\hline Conscientiousness Male (Age 10) & $\begin{array}{l}-0.009 \\
(0.872)\end{array}$ & $\begin{array}{l}0.086^{*} \\
(0.089)\end{array}$ & $\begin{array}{l}0.027 \\
(0.573)\end{array}$ & $\begin{array}{l}0.011 \\
(0.821)\end{array}$ & $\begin{array}{l}0.021 \\
(0.672)\end{array}$ \\
\hline $\begin{array}{l}\text { Emotional Stability } \\
(\text { Age 16-10) }>0.5\end{array}$ & $\begin{array}{l}-0.110 \\
(0.154)\end{array}$ & $\begin{array}{l}-0.129^{*} \\
(0.059)\end{array}$ & $\begin{array}{l}-0.136^{* *} \\
(0.032)\end{array}$ & $\begin{array}{l}-0.141^{* *} \\
(0.036)\end{array}$ & $\begin{array}{l}-0.210^{* * *} \\
(0.002)\end{array}$ \\
\hline $\begin{array}{l}\text { Emotional Stability } \\
\text { (Age 16-10) }<-0.5\end{array}$ & $\begin{array}{l}0.284^{* * *} \\
(0.000)\end{array}$ & $\begin{array}{l}0.074 \\
(0.177)\end{array}$ & $\begin{array}{l}0.101^{*} \\
(0.064)\end{array}$ & $\begin{array}{l}0.158^{* * *} \\
(0.006)\end{array}$ & $\begin{array}{l}0.234^{* * *} \\
(0.000)\end{array}$ \\
\hline $\begin{array}{l}\text { Conscientiousness } \\
(\text { Age 16-10) }>0.5\end{array}$ & $\begin{array}{l}0.009 \\
(0.909)\end{array}$ & $\begin{array}{l}-0.025 \\
(0.737)\end{array}$ & $\begin{array}{l}0.030 \\
(0.656)\end{array}$ & $\begin{array}{l}-0.044 \\
(0.536)\end{array}$ & $\begin{array}{l}-0.073 \\
(0.326)\end{array}$ \\
\hline $\begin{array}{l}\text { Conscientiousness } \\
\text { (Age 16-10) }<-0.5\end{array}$ & $\begin{array}{l}0.082 \\
(0.242)\end{array}$ & $\begin{array}{l}0.129^{* *} \\
(0.030)\end{array}$ & $\begin{array}{l}0.063 \\
(0.275)\end{array}$ & $\begin{array}{l}0.083 \\
(0.173)\end{array}$ & $\begin{array}{l}0.035 \\
(0.580)\end{array}$ \\
\hline $\begin{array}{l}\text { Emotional Stability } \\
\text { Male (Age 16-10) }>0.5\end{array}$ & $\begin{array}{l}-0.088 \\
(0.436)\end{array}$ & $\begin{array}{l}-0.035 \\
(0.718)\end{array}$ & $\begin{array}{l}0.093 \\
(0.283)\end{array}$ & $\begin{array}{l}0.009 \\
(0.925)\end{array}$ & $\begin{array}{l}0.266^{* *} \\
(0.010)\end{array}$ \\
\hline $\begin{array}{l}\text { Emotional Stability } \\
\text { Male (Age 16-10) }<-0.5\end{array}$ & $\begin{array}{l}-0.080 \\
(0.461)\end{array}$ & $\begin{array}{l}0.017 \\
(0.849)\end{array}$ & $\begin{array}{l}0.034 \\
(0.677)\end{array}$ & $\begin{array}{l}-0.047 \\
(0.592)\end{array}$ & $\begin{array}{l}-0.090 \\
(0.309)\end{array}$ \\
\hline $\begin{array}{l}\text { Conscientiousness } \\
\text { Male (Age 16-10) }>0.5\end{array}$ & $\begin{array}{l}0.107 \\
(0.369)\end{array}$ & $\begin{array}{l}0.066 \\
(0.528)\end{array}$ & $\begin{array}{l}-0.086 \\
(0.342)\end{array}$ & $\begin{array}{l}0.014 \\
(0.886)\end{array}$ & $\begin{array}{l}0.004 \\
(0.970)\end{array}$ \\
\hline $\begin{array}{l}\text { Conscientiousness } \\
\text { Male (Age 16-10) }<-0.5\end{array}$ & $\begin{array}{l}0.077 \\
(0.490)\end{array}$ & $\begin{array}{l}-0.036 \\
(0.691)\end{array}$ & $\begin{array}{l}0.119 \\
(0.156)\end{array}$ & $\begin{array}{l}0.023 \\
(0.789)\end{array}$ & $\begin{array}{l}-0.012 \\
(0.893)\end{array}$ \\
\hline Male & $\begin{array}{l}-0.341^{* * *} \\
(0.000)\end{array}$ & $\begin{array}{l}-0.358^{* * *} \\
(0.000)\end{array}$ & $\begin{array}{l}-0.294^{* * *} \\
(0.000)\end{array}$ & $\begin{array}{l}-0.240^{* * *} \\
(0.001)\end{array}$ & $\begin{array}{l}-0.266^{* * *} \\
(0.001)\end{array}$ \\
\hline $\begin{array}{l}N \\
R^{2}\end{array}$ & $\begin{array}{l}2559 \\
0.113\end{array}$ & $\begin{array}{l}3436 \\
0.100\end{array}$ & $\begin{array}{l}4200 \\
0.073\end{array}$ & $\begin{array}{l}3789 \\
0.070\end{array}$ & $\begin{array}{l}3369 \\
0.070\end{array}$ \\
\hline
\end{tabular}

Source: British Cohort Study (BCS70), own calculations.

Note: $p$-values in parentheses ${ }^{*} p<0.10,{ }^{* *} p<0.05,{ }^{* * *} p<0.01$. The OLS regressions include controls for mother's age, mother's age squared, region of living and parental socioeconomic background at birth, the personality traits agreeableness and extraversion measured at the ages of 10 and 16 as well as cognitive ability measured at the age of 10 . 


\subsubsection{Fully Restricted Sample}

Table 5.19: Mental Ill-Health: Role of Emotional Stability and Conscientiousness

\begin{tabular}{llllll}
\hline Mental Ill-Health Score $\ldots$ & Age 16 & Age 26 & Age 30 & Age 34 & Age 42 \\
\hline Emotional Stability (Age 16) & $-0.193^{* * *}$ & $-0.179^{* * *}$ & $-0.118^{* * *}$ & $-0.123^{* * *}$ & $-0.156^{* * *}$ \\
& $(0.000)$ & $(0.000)$ & $(0.002)$ & $(0.002)$ & $(0.000)$ \\
Conscientiousness (Age 16) & -0.043 & -0.048 & $-0.067^{* *}$ & $-0.079^{* *}$ & -0.030 \\
& $(0.210)$ & $(0.153)$ & $(0.048)$ & $(0.029)$ & $(0.369)$ \\
Male & $-0.295^{* * *}$ & $-0.446^{* * *}$ & $-0.338^{* * *}$ & $-0.261^{* * *}$ & $-0.252^{* * *}$ \\
& $(0.000)$ & $(0.000)$ & $(0.000)$ & $(0.000)$ & $(0.000)$ \\
\hline$N$ & 1270 & 1270 & 1270 & 1270 & 1270 \\
$R^{2}$ & 0.137 & 0.137 & 0.096 & 0.100 & 0.081 \\
\hline
\end{tabular}

Source: British Cohort Study (BCS70), own calculations.

Note: $p$-values in parentheses $* p<0.10,{ }^{*} p<0.05$, *** $p<0.01$. The OLS regressions include controls for mother's age, mother's age squared, region of living and parental socioeconomic background at birth, the personality traits agreeableness and extraversion measured at the age of 16 as well as cognitive ability measured at the age of 10 . 
Table 5.20: Substitution Effect of Conscientiousness

\begin{tabular}{|c|c|c|c|c|c|}
\hline Mental Ill-Health Score ... & Age 16 & Age 26 & Age 30 & Age 34 & Age 42 \\
\hline Emotional Stability (Age 16) & $\begin{array}{l}-0.201 * * * \\
(0.000)\end{array}$ & $\begin{array}{l}-0.177^{* * *} \\
(0.000)\end{array}$ & $\begin{array}{l}-0.140^{* * *} \\
(0.002)\end{array}$ & $\begin{array}{l}-0.172^{* *} \\
(0.000)\end{array}$ & $\begin{array}{l}-0.198^{* * *} \\
(0.000)\end{array}$ \\
\hline Conscientiousness (Age 16) & $\begin{array}{l}-0.022 \\
(0.600)\end{array}$ & $\begin{array}{l}-0.031 \\
(0.498)\end{array}$ & $\begin{array}{l}-0.103^{* *} \\
(0.043)\end{array}$ & $\begin{array}{l}-0.066 \\
(0.196)\end{array}$ & $\begin{array}{l}-0.027 \\
(0.543)\end{array}$ \\
\hline $\begin{array}{l}\text { Emotional Stability } \\
\text { Male (Age 16) }\end{array}$ & $\begin{array}{l}0.025 \\
(0.751)\end{array}$ & $\begin{array}{l}0.042 \\
(0.581)\end{array}$ & $\begin{array}{l}0.050 \\
(0.474)\end{array}$ & $\begin{array}{l}0.181^{* *} \\
(0.010)\end{array}$ & $\begin{array}{l}0.149^{* *} \\
(0.029)\end{array}$ \\
\hline $\begin{array}{l}\text { Conscientiousness } \\
\text { Male (Age 16) }\end{array}$ & $\begin{array}{l}-0.054 \\
(0.446)\end{array}$ & $\begin{array}{l}0.002 \\
(0.969)\end{array}$ & $\begin{array}{l}0.070 \\
(0.301)\end{array}$ & $\begin{array}{l}-0.013 \\
(0.862)\end{array}$ & $\begin{array}{l}-0.002 \\
(0.972)\end{array}$ \\
\hline $\begin{array}{l}\text { Emotional Stability } \times \\
\text { Conscientiousness (Age 16) }\end{array}$ & $\begin{array}{l}0.002 \\
(0.941)\end{array}$ & $\begin{array}{l}0.024 \\
(0.371)\end{array}$ & $\begin{array}{l}-0.019 \\
(0.410)\end{array}$ & $\begin{array}{l}0.003 \\
(0.915)\end{array}$ & $\begin{array}{l}-0.007 \\
(0.806)\end{array}$ \\
\hline $\begin{array}{l}\text { Emotional Stability } \times \\
\text { Conscientiousness } \times \\
\text { Male }(\text { Age } 16)\end{array}$ & $\begin{array}{l}-0.003 \\
(0.947)\end{array}$ & $\begin{array}{l}0.017 \\
(0.766)\end{array}$ & $\begin{array}{l}0.010 \\
(0.853)\end{array}$ & $\begin{array}{l}0.033 \\
(0.593)\end{array}$ & $\begin{array}{l}0.041 \\
(0.436)\end{array}$ \\
\hline Male & $\begin{array}{l}-0.295^{* * *} \\
(0.000)\end{array}$ & $\begin{array}{l}-0.451^{* * *} \\
(0.000)\end{array}$ & $\begin{array}{l}-0.345^{* * *} \\
(0.000)\end{array}$ & $\begin{array}{l}-0.281^{* * *} \\
(0.000)\end{array}$ & $\begin{array}{l}-0.276^{* * *} \\
(0.000)\end{array}$ \\
\hline$N$ & 1270 & 1270 & 1270 & 1270 & 1270 \\
\hline$R^{2}$ & 0.137 & 0.139 & 0.098 & 0.105 & 0.084 \\
\hline
\end{tabular}

Source: British Cohort Study (BCS70), own calculations.

Note: $p$-values in parentheses ${ }^{*} p<0.10,{ }^{* *} p<0.05,{ }^{* * *} p<0.01$. The OLS regressions include controls for mother's age, mother's age squared, region of living and parental socioeconomic background at birth, the personality traits agreeableness and extraversion measured at the age of 16 as well as cognitive ability measured at the age of 10 . 
Table 5.21: Personality Types and Mental Ill-Health

\begin{tabular}{llllll}
\hline Mental Ill-Health Score ... & Age 16 & Age 26 & Age 30 & Age 34 & Age 42 \\
\hline High Emotional Stability, & 0.063 & 0.027 & $0.151^{*}$ & 0.032 & -0.002 \\
Low Conscientiousness (Age 16) & $(0.479)$ & $(0.742)$ & $(0.087)$ & $(0.714)$ & $(0.979)$ \\
Low Emotional Stability, & $0.273^{* * *}$ & 0.069 & 0.073 & 0.037 & 0.113 \\
High Conscientiousness (Age 16) & $(0.001)$ & $(0.380)$ & $(0.370)$ & $(0.654)$ & $(0.142)$ \\
Low Emotional Stability, & $0.337^{* * *}$ & $0.285^{* * *}$ & $0.326^{* * *}$ & $0.400^{* * *}$ & $0.361^{* * *}$ \\
Low Conscientiousness (Age 16) & $(0.000)$ & $(0.002)$ & $(0.001)$ & $(0.000)$ & $(0.000)$ \\
Male & $-0.305^{* * * *}$ & $-0.460^{* * *}$ & $-0.349^{* * *}$ & $-0.268^{* * *}$ & $-0.263^{* * *}$ \\
& $(0.000)$ & $(0.000)$ & $(0.000)$ & $(0.000)$ & $(0.000)$ \\
\hline$N$ & 1270 & 1270 & 1270 & 1270 & 1270 \\
$R^{2}$ & 0.125 & 0.121 & 0.092 & 0.099 & 0.077 \\
\hline
\end{tabular}

Source: British Cohort Study (BCS70), own calculations.

Note: $p$-values in parentheses ${ }^{*} p<0.10,{ }^{* *} p<0.05,{ }^{* * *} p<0.01$. The reference group for the personality types comprises emotionally stable and highly conscientious individuals. The OLS regressions include controls for mother's age, mother's age squared, region of living and parental socioeconomic background at birth, the personality traits agreeableness and extraversion measured at the age of 16 as well as cognitive ability measured at the age of 10 .

Table 5.22: Role of Emotional Stability and Conscientiousness - Controlling for Mental Ill-Health at Age 16

\begin{tabular}{lllll}
\hline Mental Ill-Health Score ... & Age 26 & Age 30 & Age 34 & Age 42 \\
\hline Emotional Stability (Age 16) & $-0.117^{* * *}$ & $-0.065^{*}$ & $-0.069^{*}$ & $-0.119^{* * *}$ \\
& $(0.001)$ & $(0.067)$ & $(0.069)$ & $(0.002)$ \\
Conscientiousness (Age 16) & -0.034 & $-0.055^{*}$ & $-0.067^{*}$ & -0.022 \\
& $(0.277)$ & $(0.086)$ & $(0.050)$ & $(0.509)$ \\
Malaise Score (Age 16) & $0.119^{* * *}$ & $0.103^{* * *}$ & $0.105^{* * *}$ & $0.071^{* * *}$ \\
& $(0.000)$ & $(0.000)$ & $(0.000)$ & $(0.000)$ \\
Male & $-0.351^{* * *}$ & $-0.256^{* * *}$ & $-0.177^{* * *}$ & $-0.196^{* * *}$ \\
& $(0.000)$ & $(0.000)$ & $(0.001)$ & $(0.001)$ \\
\hline$N$ & 1270 & 1270 & 1270 & 1270 \\
$R^{2}$ & 0.225 & 0.162 & 0.169 & 0.113 \\
\hline
\end{tabular}

Source: British Cohort Study (BCS70), own calculations.

Note: $p$-values in parentheses $* p<0.10,{ }^{* *} p<0.05$, *** $p<0.01$. The OLS regressions include controls for mother's age, mother's age squared, region of living and parental socioeconomic background at birth, the personality traits agreeableness and extraversion measured at the age of 16 as well as cognitive ability measured at the age of 10 . 
Table 5.23: Personality Types and Mental Ill-Health - Controlling for Mental Health at Age 16

\begin{tabular}{lllll}
\hline Mental Ill-Health Score ... & Age 26 & Age 30 & Age 34 & Age 42 \\
\hline High Emotional Stability, & 0.006 & 0.133 & 0.013 & -0.015 \\
Low Conscientiousness (Age 16) & $(0.939)$ & $(0.126)$ & $(0.876)$ & $(0.864)$ \\
Low Emotional Stability, & -0.022 & -0.005 & -0.043 & 0.059 \\
High Conscientiousness (Age 16) & $(0.762)$ & $(0.951)$ & $(0.590)$ & $(0.451)$ \\
Low Emotional Stability, & $0.172^{*}$ & $0.230^{* *}$ & $0.302^{* * *}$ & $0.294^{* * *}$ \\
Low Conscientiousness (Age 16) & $(0.051)$ & $(0.013)$ & $(0.001)$ & $(0.002)$ \\
Malaise Score (Age 16) & $0.124^{* * *}$ & $0.106^{* * *}$ & $0.108^{* * *}$ & $0.074^{* * *}$ \\
& $(0.000)$ & $(0.000)$ & $(0.000)$ & $(0.000)$ \\
Male & $-0.358^{* * *}$ & $-0.262^{* * *}$ & $-0.180^{* * *}$ & $-0.202^{* * *}$ \\
& $(0.000)$ & $(0.000)$ & $(0.001)$ & $(0.000)$ \\
\hline$N$ & 1270 & 1270 & 1270 & 1270 \\
$R^{2}$ & 0.219 & 0.163 & 0.173 & 0.112 \\
\hline
\end{tabular}

Source: British Cohort Study (BCS70), own calculations.

Note: $p$-values in parentheses ${ }^{*} p<0.10,{ }^{* *} p<0.05,{ }^{* * *} p<0.01$. The reference group for the personality types comprises emotionally stable and highly conscientious individuals. The OLS regressions include controls for mother's age, mother's age squared, region of living and parental socioeconomic background at birth, the personality traits agreeableness and extraversion measured at the age of 16 as well as cognitive ability measured at the age of 10 . 
Table 5.24: Personality Trait Change and Mental Ill-Health

\begin{tabular}{llllll}
\hline Mental Ill-Health Score ... & Age 16 & Age 26 & Age 30 & Age 34 & Age 42 \\
\hline Emotional Stability (Age 10) & $-0.187^{* * *}$ & $-0.148^{* * *}$ & $-0.104^{* *}$ & $-0.130^{* * *}$ & $-0.114^{* *}$ \\
& $(0.000)$ & $(0.003)$ & $(0.022)$ & $(0.004)$ & $(0.012)$ \\
Conscientiousness (Age 10) & 0.025 & -0.039 & -0.044 & $-0.103^{* *}$ & $-0.094^{* *}$ \\
& $(0.521)$ & $(0.330)$ & $(0.283)$ & $(0.012)$ & $(0.017)$ \\
Emotional Stability & $-0.186^{* *}$ & $-0.204^{* *}$ & -0.035 & $-0.244^{* * *}$ & $-0.184^{* *}$ \\
(Age 16-10) $\rangle 0.5$ & $(0.022)$ & $(0.012)$ & $(0.684)$ & $(0.005)$ & $(0.033)$ \\
Emotional Stability & $0.235^{* * *}$ & $0.155^{* *}$ & 0.054 & $0.124^{*}$ & $0.209^{* * *}$ \\
Age 16-10) $\langle-0.5$ & $(0.002)$ & $(0.029)$ & $(0.465)$ & $(0.095)$ & $(0.005)$ \\
Conscientiousness & -0.015 & -0.036 & 0.034 & -0.026 & -0.046 \\
(Age 16-10) $\rangle 0.5$ & $(0.850)$ & $(0.665)$ & $(0.680)$ & $(0.754)$ & $(0.592)$ \\
Conscientiousness & $0.154^{* *}$ & 0.037 & $0.187^{* *}$ & 0.042 & 0.032 \\
$($ Age 16-10) $\langle-0.5$ & $(0.043)$ & $(0.620)$ & $(0.014)$ & $(0.581)$ & $(0.669)$ \\
Male & $-0.288^{* * *}$ & $-0.460^{* * *}$ & $-0.347^{* * *}$ & $-0.276^{* * *}$ & $-0.275^{* * *}$ \\
\hline$N$ & $(0.000)$ & $(0.000)$ & $(0.000)$ & $(0.000)$ & $(0.000)$ \\
$R^{2}$ & 1270 & 1270 & 1270 & 1270 & 1270 \\
\hline
\end{tabular}

Source: British Cohort Study (BCS70), own calculations.

Note: $p$-values in parentheses $* p<0.10,{ }^{* *} p<0.05, * * * p<0.01$. The OLS regressions include controls for mother's age, mother's age squared, region of living and parental socioeconomic background at birth, the personality traits agreeableness and extraversion measured at the ages of 10 and 16 as well as cognitive ability measured at the age of 10 . 


\subsubsection{Imputation of Missing Values}

Table 5.25: Role of Emotional Stability and Conscientiousness

\begin{tabular}{llllll}
\hline Mental Ill-Health Score ... & Age 16 & Age 26 & Age 30 & Age 34 & Age 42 \\
\hline Emotional Stability (Age 16) & $-0.154^{* * *}$ & $-0.096^{* * *}$ & $-0.116^{* * *}$ & $-0.080^{* * *}$ & $-0.111^{* * *}$ \\
& $(0.000)$ & $(0.000)$ & $(0.000)$ & $(0.000)$ & $(0.000)$ \\
Conscientiousness (Age 16) & -0.017 & $-0.073^{* * *}$ & $-0.069^{* * *}$ & $-0.083^{* * *}$ & -0.019 \\
& $(0.442)$ & $(0.000)$ & $(0.000)$ & $(0.000)$ & $(0.321)$ \\
Male & $-0.330^{* * *}$ & $-0.383^{* * *}$ & $-0.257^{* * *}$ & $-0.243^{* * *}$ & $-0.199^{* * *}$ \\
& $(0.000)$ & $(0.000)$ & $(0.000)$ & $(0.000)$ & $(0.000)$ \\
\hline$N$ & 3142 & 4066 & 4910 & 4399 & 3878 \\
$R^{2}$ & 0.104 & 0.104 & 0.075 & 0.069 & 0.067 \\
\hline
\end{tabular}

Source: British Cohort Study (BCS70), own calculations.

Note: $p$-values in parentheses ${ }^{*} p<0.10,{ }^{* *} p<0.05,{ }^{* * *} p<0.01$. The OLS regressions include controls for mother's age, mother's age squared, region of living and parental socioeconomic background at birth, the personality traits agreeableness and extraversion measured at the age of 16 as well as cognitive ability measured at the age of 10 . 
Table 5.26: Substitution Effect of Conscientiousness

\begin{tabular}{|c|c|c|c|c|c|}
\hline Mental Ill-Health Score ... & Age 16 & Age 26 & Age 30 & Age 34 & Age 42 \\
\hline Emotional Stability (Age 16) & $\begin{array}{l}-0.184^{* * *} \\
(0.000)\end{array}$ & $\begin{array}{l}-0.091^{* * *} \\
(0.001)\end{array}$ & $\begin{array}{l}-0.126^{* * *} \\
(0.000)\end{array}$ & $\begin{array}{l}-0.093^{* * *} \\
(0.001)\end{array}$ & $\begin{array}{l}-0.132^{* * *} \\
(0.000)\end{array}$ \\
\hline Conscientiousness (Age 16) & $\begin{array}{l}-0.004 \\
(0.905)\end{array}$ & $\begin{array}{l}-0.116^{* * *} \\
(0.000)\end{array}$ & $\begin{array}{l}-0.076^{* *} \\
(0.018)\end{array}$ & $\begin{array}{l}-0.115^{* * *} \\
(0.000)\end{array}$ & $\begin{array}{l}-0.040 \\
(0.164)\end{array}$ \\
\hline $\begin{array}{l}\text { Emotional Stability } \\
\text { Male (Age 16) }\end{array}$ & $\begin{array}{l}0.067 \\
(0.151)\end{array}$ & $\begin{array}{l}-0.010 \\
(0.788)\end{array}$ & $\begin{array}{l}0.014 \\
(0.690)\end{array}$ & $\begin{array}{l}0.025 \\
(0.538)\end{array}$ & $\begin{array}{l}0.044 \\
(0.281)\end{array}$ \\
\hline $\begin{array}{l}\text { Conscientiousness } \\
\text { Male (Age 16) }\end{array}$ & $\begin{array}{l}-0.037 \\
(0.402)\end{array}$ & $\begin{array}{l}0.070^{*} \\
(0.078)\end{array}$ & $\begin{array}{l}0.006 \\
(0.871)\end{array}$ & $\begin{array}{l}0.039 \\
(0.353)\end{array}$ & $\begin{array}{l}0.027 \\
(0.504)\end{array}$ \\
\hline $\begin{array}{l}\text { Emotional Stability } \times \\
\text { Conscientiousness (Age 16) }\end{array}$ & $\begin{array}{l}0.002 \\
(0.926)\end{array}$ & $\begin{array}{l}-0.019 \\
(0.311)\end{array}$ & $\begin{array}{l}-0.010 \\
(0.551)\end{array}$ & $\begin{array}{l}-0.033^{*} \\
(0.052)\end{array}$ & $\begin{array}{l}-0.022 \\
(0.190)\end{array}$ \\
\hline $\begin{array}{l}\text { Emotional Stability } \times \\
\text { Conscientiousness } \times \\
\text { Male (Age 16) }\end{array}$ & $\begin{array}{l}-0.017 \\
(0.635)\end{array}$ & $\begin{array}{l}0.016 \\
(0.555)\end{array}$ & $\begin{array}{l}0.000 \\
(0.986)\end{array}$ & $\begin{array}{l}0.041 \\
(0.113)\end{array}$ & $\begin{array}{l}0.025 \\
(0.336)\end{array}$ \\
\hline Male & $\begin{array}{l}-0.326^{* * *} \\
(0.000)\end{array}$ & $\begin{array}{l}-0.395^{* * *} \\
(0.000)\end{array}$ & $\begin{array}{l}-0.258^{* * *} \\
(0.000)\end{array}$ & $\begin{array}{l}-0.265^{* * *} \\
(0.000)\end{array}$ & $\begin{array}{l}-0.215^{* * *} \\
(0.000)\end{array}$ \\
\hline $\begin{array}{l}N \\
R^{2}\end{array}$ & $\begin{array}{l}3142 \\
0.104\end{array}$ & $\begin{array}{l}4066 \\
0.105\end{array}$ & $\begin{array}{l}4910 \\
0.074\end{array}$ & $\begin{array}{l}4399 \\
0.071\end{array}$ & $\begin{array}{l}3878 \\
0.067\end{array}$ \\
\hline
\end{tabular}

Source: British Cohort Study (BCS70), own calculations.

Note: $p$-values in parentheses $* p<0.10,{ }^{* *} p<0.05, * * * p<0.01$. The OLS regressions include controls for mother's age, mother's age squared, region of living and parental socioeconomic background at birth, the personality traits agreeableness and extraversion measured at the age of 16 as well as cognitive ability measured at the age of 10 . 
Table 5.27: Personality Types and Mental Ill-Health

\begin{tabular}{llllll}
\hline Mental Ill-Health Score ... & Age 16 & Age 26 & Age 30 & Age 34 & Age 42 \\
\hline High Emotional Stability, & 0.063 & $0.100^{* *}$ & $0.145^{* * *}$ & $0.109^{* *}$ & 0.028 \\
Low Conscientiousness (Age 16) & $(0.259)$ & $(0.040)$ & $(0.001)$ & $(0.017)$ & $(0.574)$ \\
Low Emotional Stability, & $0.202^{* * *}$ & $0.090^{* *}$ & $0.088^{* *}$ & $0.093^{* *}$ & $0.134^{* * *}$ \\
High Conscientiousness (Age 16) & $(0.000)$ & $(0.033)$ & $(0.028)$ & $(0.027)$ & $(0.003)$ \\
Low Emotional Stability, & $0.269^{* * *}$ & $0.266^{* * *}$ & $0.308^{* * *}$ & $0.282^{* * *}$ & $0.235^{* * *}$ \\
Low Conscientiousness (Age 16) & $(0.000)$ & $(0.000)$ & $(0.000)$ & $(0.000)$ & $(0.000)$ \\
Male & $-0.345^{* * *}$ & $-0.392^{* * *}$ & $-0.273^{* * *}$ & $-0.250^{* * *}$ & $-0.211^{* * *}$ \\
& $(0.000)$ & $(0.000)$ & $(0.000)$ & $(0.000)$ & $(0.000)$ \\
\hline$N$ & 3142 & 4066 & 4910 & 4399 & 3878 \\
$R^{2}$ & 0.097 & 0.099 & 0.071 & 0.066 & 0.065 \\
\hline
\end{tabular}

Source: British Cohort Study (BCS70), own calculations.

Note: $p$-values in parentheses ${ }^{*} p<0.10,{ }^{* *} p<0.05,{ }^{* * *} p<0.01$. The reference group for the personality types comprises emotionally stable and highly conscientious individuals. The OLS regressions include controls for mother's age, mother's age squared, region of living and parental socioeconomic background at birth, the personality traits agreeableness and extraversion measured at the age of 16 as well as cognitive ability measured at the age of 10 .

Table 5.28: Role of Emotional Stability and Conscientiousness - Controlling for Mental Ill-Health at Age 16

\begin{tabular}{lllll}
\hline Mental Ill-Health Score ... & Age 26 & Age 30 & Age 34 & Age 42 \\
\hline Emotional Stability (Age 16) & $-0.110^{* * *}$ & $-0.099^{* * *}$ & $-0.056^{* *}$ & $-0.154^{* * *}$ \\
& $(0.000)$ & $(0.000)$ & $(0.047)$ & $(0.000)$ \\
Conscientiousness (Age 16) & $-0.063^{* *}$ & $-0.040^{*}$ & $-0.094^{* * *}$ & -0.018 \\
& $(0.016)$ & $(0.097)$ & $(0.001)$ & $(0.487)$ \\
Malaise Score (Age 16) & $0.111^{* * *}$ & $0.098^{* * *}$ & $0.096^{* * *}$ & $0.075^{* * *}$ \\
& $(0.000)$ & $(0.000)$ & $(0.000)$ & $(0.000)$ \\
Male & $-0.304^{* * *}$ & $-0.177^{* * *}$ & $-0.164^{* * *}$ & $-0.177^{* * *}$ \\
& $(0.000)$ & $(0.000)$ & $(0.000)$ & $(0.000)$ \\
\hline$N$ & 2362 & 2615 & 2405 & 2176 \\
$R^{2}$ & 0.205 & 0.152 & 0.145 & 0.132 \\
\hline
\end{tabular}

Source: British Cohort Study (BCS70), own calculations.

Note: $p$-values in parentheses ${ }^{*} p<0.10,{ }^{* *} p<0.05,{ }^{* * *} p<0.01$. The OLS regressions include controls for mother's age, mother's age squared, region of living and parental socioeconomic background at birth, the personality traits agreeableness and extraversion measured at the age of 16 as well as cognitive ability measured at the age of 10 . 
Table 5.29: Personality Types and Mental Ill-Health - Controlling for Mental IllHealth at Age 16

\begin{tabular}{lllll}
\hline Mental Ill-Health Score... & Age 26 & Age 30 & Age 34 & Age 42 \\
\hline High Emotional Stability, & 0.083 & 0.075 & $0.114^{*}$ & 0.019 \\
Low Conscientiousness (Age 16) & $(0.195)$ & $(0.202)$ & $(0.071)$ & $(0.759)$ \\
Low Emotional Stability, & $0.104^{* *}$ & 0.062 & 0.047 & $0.148^{* * *}$ \\
High Conscientiousness (Age 16) & $(0.049)$ & $(0.226)$ & $(0.370)$ & $(0.008)$ \\
Low Emotional Stability, & $0.274^{* * *}$ & $0.237^{* * *}$ & $0.241^{* * *}$ & $0.309^{* * *}$ \\
Low Conscientiousness (Age 16) & $(0.000)$ & $(0.000)$ & $(0.000)$ & $(0.000)$ \\
Malaise Score (Age 16) & $0.114^{* * *}$ & $0.100^{* * *}$ & $0.098^{* * *}$ & $0.079^{* * *}$ \\
& $(0.000)$ & $(0.000)$ & $(0.000)$ & $(0.000)$ \\
Male & $-0.310^{* * *}$ & $-0.185^{* * *}$ & $-0.166^{* * *}$ & $-0.188^{* * *}$ \\
\hline$N$ & $(0.000)$ & $(0.000)$ & $(0.000)$ & $(0.000)$ \\
\hline$R^{2}$ & 2362 & 2615 & 2405 & 2176 \\
\hline
\end{tabular}

Source: British Cohort Study (BCS70), own calculations.

Note: $p$-values in parentheses ${ }^{*} p<0.10,{ }^{* *} p<0.05,{ }^{* * *} p<0.01$. The reference group for the personality types comprises emotionally stable and highly conscientious individuals. The OLS regressions include controls for mother's age, mother's age squared, region of living and parental socioeconomic background at birth, the personality traits agreeableness and extraversion measured at the age of 16 as well as cognitive ability measured at the age of 10 . 
Table 5.30: Personality Trait Change and Mental Ill-Health

\begin{tabular}{llllll}
\hline Mental Ill-Health Score $\ldots$ & Age 16 & Age 26 & Age 30 & Age 34 & Age 42 \\
\hline Emotional Stability (Age 10) & $-0.106^{* * *}$ & $-0.096^{* * *}$ & $-0.062^{* * *}$ & $-0.077^{* * *}$ & $-0.067^{* * *}$ \\
& $(0.000)$ & $(0.000)$ & $(0.006)$ & $(0.002)$ & $(0.009)$ \\
Conscientiousness (Age 10) & 0.010 & $-0.045^{*}$ & -0.032 & $-0.075^{* * *}$ & $-0.058^{* *}$ \\
& $(0.686)$ & $(0.055)$ & $(0.141)$ & $(0.002)$ & $(0.015)$ \\
Emotional Stability & $-0.158^{* * *}$ & $-0.137^{* * *}$ & -0.062 & $-0.131^{* * *}$ & -0.077 \\
(Age 16-10) $\rangle 0.5$ & $(0.001)$ & $(0.002)$ & $(0.126)$ & $(0.002)$ & $(0.101)$ \\
Emotional Stability & $0.224^{* * *}$ & $0.080^{*}$ & $0.103^{* * *}$ & $0.132^{* * *}$ & $0.219^{* * *}$ \\
Age 16-10) $\langle-0.5$ & $(0.000)$ & $(0.050)$ & $(0.006)$ & $(0.001)$ & $(0.000)$ \\
Conscientiousness & 0.032 & -0.016 & 0.006 & -0.020 & -0.059 \\
$($ Age 16-10) $\rangle 0.5$ & $(0.543)$ & $(0.742)$ & $(0.882)$ & $(0.673)$ & $(0.233)$ \\
Conscientiousness & 0.073 & $0.095^{* *}$ & $0.146^{* * *}$ & $0.098^{* *}$ & 0.048 \\
$($ Age 16-10) $\langle-0.5$ & $(0.138)$ & $(0.020)$ & $(0.000)$ & $(0.015)$ & $(0.257)$ \\
Male & $-0.336^{* * *}$ & $-0.393^{* * *}$ & $-0.275^{* * *}$ & $-0.250^{* * *}$ & $-0.213^{* * *}$ \\
\hline$N$ & $(0.000)$ & $(0.000)$ & $(0.000)$ & $(0.000)$ & $(0.000)$ \\
$R^{2}$ & 3142 & 4066 & 4910 & 4399 & 3878 \\
\hline
\end{tabular}

Source: British Cohort Study (BCS70), own calculations.

Note: $p$-values in parentheses ${ }^{*} p<0.10,{ }^{* *} p<0.05,{ }^{* * *} p<0.01$. The OLS regressions include controls for mother's age, mother's age squared, region of living and parental socioeconomic background at birth, the personality traits agreeableness and extraversion measured at the ages of 10 and 16 as well as cognitive ability measured at the age of 10 . 
CHAPTER 6

PESSIMISTIC EXPECTATIONS AND

MENTAL HEALTH 


\subsection{Introduction}

Given the association between emotional stability, conscientiousness and mental health problems, a question is whether and to what extent pessimistic expectations play a role in these relations. It is investigated by analyzing the relation (1) between the personality traits emotional stability and conscientiousness at the ages of 10 and 16 and pessimistic expectations at the age of $16,(2)$ between pessimistic expectations at the age of 16 and mental ill-health at later ages and (3) between a change in pessimistic expectations between the ages of 16 and 30 and mental health problems at later ages.

In Chapter 2, pessimistic expectations are hypothesized to be positively related to mental ill-health, because they are likely to reduce the individual perceived and actual problem-solving ability in influenceable, challenging situations. That way, the underlying problem is likely to persist which might be related to unhealthy sustained stress. On the one hand, feelings of stress persist until a challenge is at least mentally solved. This is less likely for pessimistic individuals. On the other hand, pessimistic expectations are likely to translate into lower effort invested to solve the problem which might result in worsened outcomes and a further rise in anxiety. A vicious circle based on negative expectations and negative experiences is completed and likely to be sustained. Assuming that individuals are confronted with various smaller and larger challenges day-by-day, situations where feelings of sustained stress related to destructive thoughts and avoidance behavior may occur are likely to cumulate. This might result in mental health problems in the long run.

Based on the theoretical framework, it is hypothesized that pessimistic expectations partially mediate the relation between particularly emotional stability and mental health outcomes. Mediation means that thoughts of helplessness and hopelessness as indicators of pessimistic thoughts are related to both emotional stability and mental health problems, whereby pessimistic expectations account for some of the relation between emotional stability and mental health problems. However, partial mediation means that both personality traits and pessimistic thoughts also have an independent direct association with mental ill-health. Accordingly, also environmental factors such as social and economic conditions are likely to influence beliefs and expectations.

Chapter 5 suggests that particularly both low emotionally stable and low consci- 
entious individuals have a higher risk of experiencing mental ill-health throughout life which is in line with the theoretical considerations. However, while personality traits can be regarded as more stable and more difficult to change, expectations are considered more volatile and adjustable (Roberts, 2009). Economic insights into the role of pessimistic expectations in explaining mental health outcomes, above and beyond personality might help to further determine high-risk groups and investigate a potential starting point for effective and efficient school programmes.

Chapter 4 shows that pessimistic expectations regarding one own's ability in exams at the age of 16 are predictive for education outcomes at the age of 30 for both women and men. Individuals without pessimistic expectations in exams reach significantly better outcomes compared to the pessimistic ones also after controlling for cognitive ability. This is in line with the theoretical considerations and indicates that pessimistic expectations are likely to be one important channel for educational achievement. This chapter analyzes whether pessimistic expectations also play a role for mental health outcomes throughout life. In the following, the data, the results and a conclusion are presented.

\subsection{Data}

The study makes use of the BCS70, which provides detailed information about the individual's family background, cognitive ability, personality and mental health problems as well as thoughts of helplessness and hopelessness indicating inefficient and maladaptive coping strategies. Chapter 3 Appendix Table 3.6 provides an overview of the BCS70 datasets used in the analysis, variables investigated and the overall number of observations in each of the eight waves.

The initial number of observations presented in Chapter 3 Appendix Table 3.6 is not congruent with the actual number of observations used in the analysis due to panel attrition and missing values within the variables of interest. The working sample is restricted to cases with available information on the personality traits emotional stability, conscientiousness, agreeableness, and extraversion at the ages of 10 and 16 , indicators for thoughts of helplessness and hopelessness, cognitive ability at the age of 10, mother's age, parental socioeconomic status and region of living at birth as well as one mental ill-health score at a time, resulting in five separate samples with 2051 (age 16), 1634 (age 26), 2050 (age 30), 1783 (age 34) and 1592 (age 42) 
observations. The issue of sample selection is addressed by descriptively comparing the working samples with the unrestricted samples (Appendix Subsection 6.5.1). The comparison shows that the working samples are positively selected. This makes it likely to estimate lower bound results and that the observed associations are even larger in the unrestricted sample. For a more detailed discussion see Chapter 3 Subsection 3.2 .

To test the robustness of the results, two checks are conducted. First, all calculations are repeated based on a fully restricted sample, which restricts the availability of all variables used and includes 1,218 observations. The results are consistent with the main results and are available in Appendix Subsection 6.5.2.2. Second, multiple imputation of missing values regarding the personality trait variables and the indicator of mental health problems is conducted. Personality item are imputed if there are five or fewer missing values out of 22 items. Malaise items are imputed if there are three or fewer missing values out of nine items. Imputation of missing values results in an increase of the sample size to 2309 (age 16), 1832 (age 26), 2308 (age 30), 1999 (age 34) and 1775 (age 42) observations. The results are consistent with the main results and available in Appendix Subsection 6.5.2.3. In the following, all variables are discussed in detail.

\subsubsection{Mental Health Outcomes}

An indicator of mental health problems is assessed by Rutter et al. (1970)'s Malaise Inventory that is measured at the ages of $16,26,30,34$, and 42 and that covers emotional disturbance and associated physical symptoms (UK Data Service, 2016). Figure 6.1 depicts the distribution of mental health problems among men and women. A detailed description and discussion of the variable is provided in Chapter 5 Subsection 5.2.1.

\subsubsection{Pessimistic Expectations}

As indicators of pessimistic expectations, thoughts of helplessness and hopelessness are considered (Ursin and Eriksen, 2004). "Helplessness is the belief that there is nothing the individuals can do to change their lives" (Vatan and Lester, 2008, p. 701). "Hopelessness is a cognitive attitude in which people are not optimistic and 
Figure 6.1: Mental Ill-Health at Ages 16, 26, 30, 34 and 42

Age 16

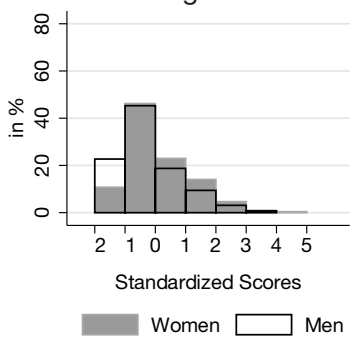

Age 34

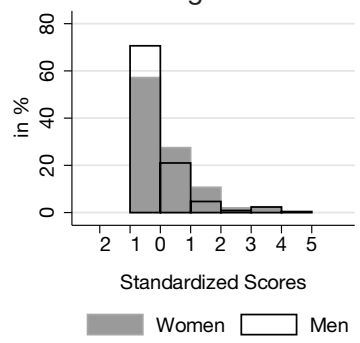

Age 26

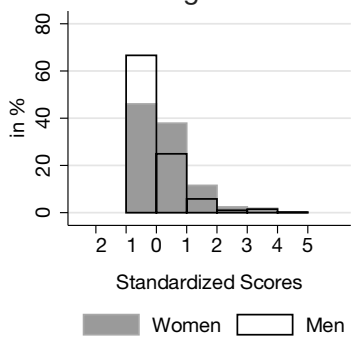

Age 42

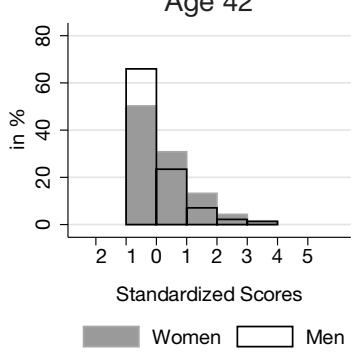

Age 30

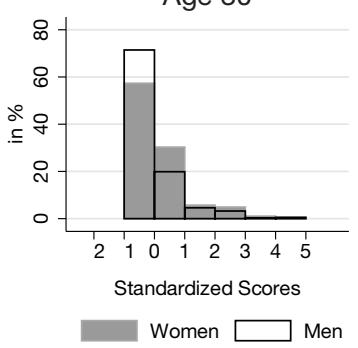

Source: BCS70. Own calculations, $\mathrm{n}=2051$ (age 16), $\mathrm{n}=1634$ (age 26), $\mathrm{n}=2050$ (age 30), $\mathrm{n}=$ 1783 (age 34$), \mathrm{n}=1592$ (age 42).

have lost motivation about their future" (Vatan and Lester, 2008, p. 701). Lester (2001) provides an inventory to measure helplessness and hopelessness. Question to measure the concept of helplessness include "I don't seem to be able to cope with crisis without the help of others", "I can hardly find ways around problems that I face" or "There are few ways around the problems that I am facing now" (Lester, 2001, p. 498). Hopelessness is measured by "I don't expect to get what I really want", "It is very unlikely that I will get any real satisfaction in the future" or "I never get what I want, so it's foolish to want anything" (Lester, 2001, p. 498).

The BCS70 does not provide these questions. However, there are two questions available that are very similar to those in the inventory provided by Lester (2001). Helplessness is operationalized by the variable "I cannot overcome my difficulties" at the ages of 16 and 30 . The variable has the answer categories 1 "not at all," 2 "no more than usual," 3 "rather more than usual" and 4 "much more than usual." Hopelessness is operationalized by the variable "I'm not really getting anywhere 
Figure 6.2: Pessimistic Expectations at Age 16
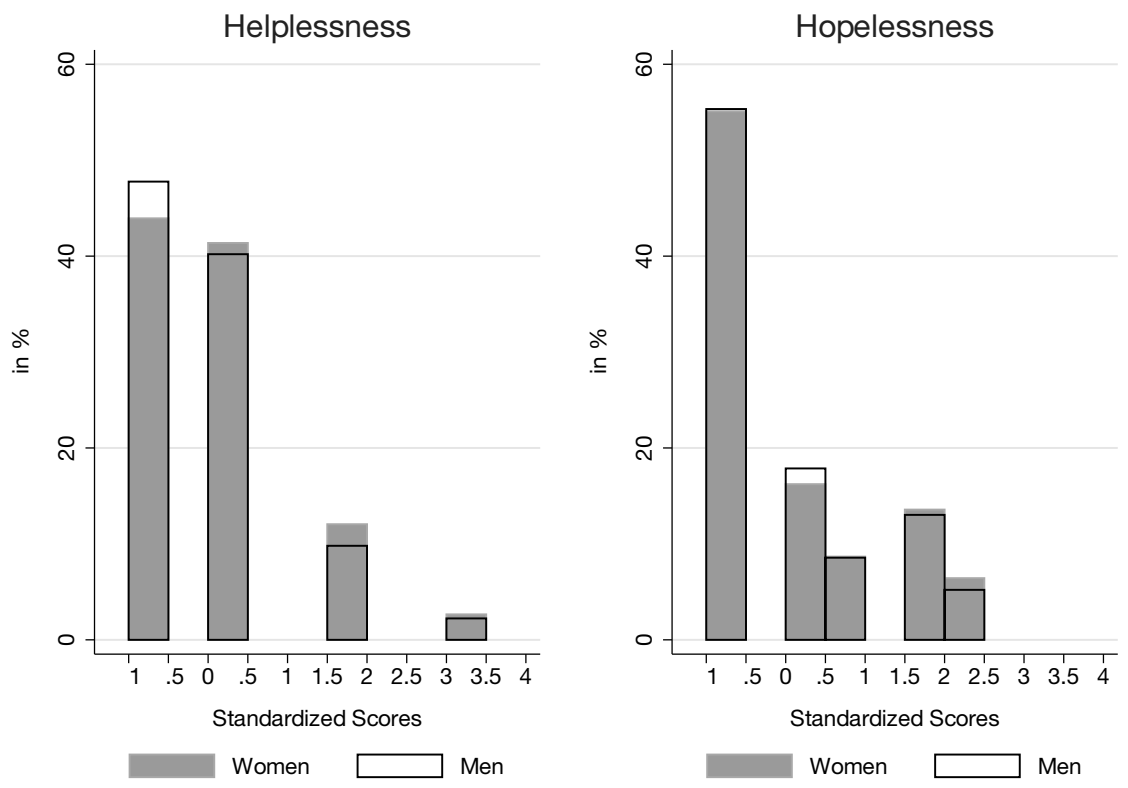

Source: BCS70. Own calculations, $\mathrm{n}=2051$.

with my life" at the age of 16 . It has the answer categories 1 "disagree a lot," 2 "disagree a little," 3 "not sure," 4 "agree a little" and 5 "agree a lot." For both variables, the categories "not stated" and "no questionnaire" are coded as missing values.

Figure 6.2 presents the distribution of helplessness and hopelessness among females and males. It depicts that females more often experience thoughts of helplessness than males, while the distribution of hopelessness is similar for both genders. ${ }^{15}$

In the analysis, the helplessness and hopelessness variables are recoded into binary variables. The variable helplessness is recoded 0 if the respondent chooses the answer category 1 "not at all" and 1 if the respondent answers 2 "no more than usual," 3 "rather more than usual" or 4 "much more than usual." Hopelessness is recoded 0 if

15 Please note that in this section, males are significantly less helpless than females. However, in the fully restricted sample the gender dummy is no longer significant, which is in line with the argumentation of positive sample selection. 
the respondent takes the answer category 1 "disagree a lot" or 2 "disagree a little." It is recoded into 1 if the individual says 3 "not sure," 4 "agree a little," or 5 "agree a lot."

\subsubsection{Personality Traits}

Figure 6.3: Personality Traits at Ages 10 and 16
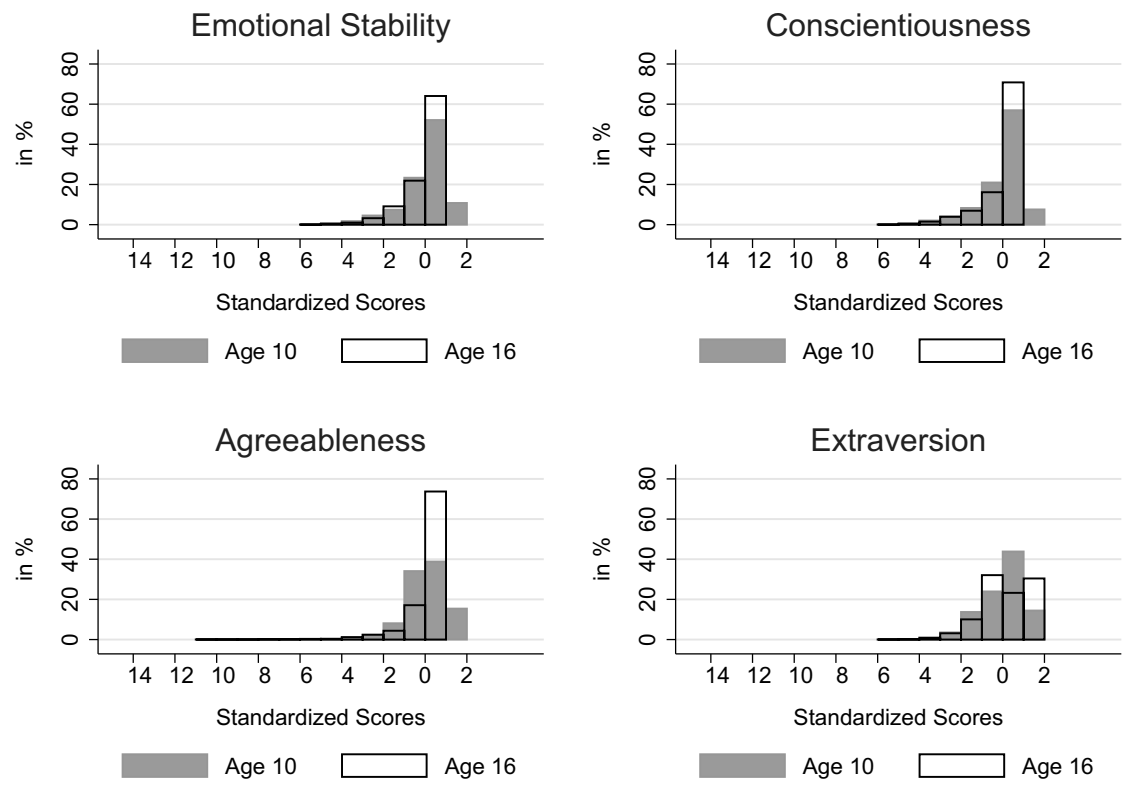

Source: BCS70. Own calculations, $\mathrm{n}=2051$.

In the analysis, personality traits at the ages of 10 and 16 are used threefold: First, they are used as continues variables as shown in Figure 6.3. Second, four personality types capturing differences in emotional stability and conscientiousness are created. Thereby, the distribution of the standardized variables emotional stability and conscientiousness is split at the mean, i.e. at zero, and four above- and below-mean combinations of emotional stability and conscientiousness are constructed: (1) more emotionally stable and more conscientious, (2) more emotionally stable and less conscientious, (3) less emotionally stable and more conscientious, and (4) less emotionally stable and less conscientious individuals. Third, the change in personality 
between the ages of 10 and 16 is used by subtracting the standardized personality score at the age of 10 from the standardized score at the age of 16 .

A detailed discussion about the personality traits used in the analysis is provided in Chapter 3 Subsection 3.2.2. In this chapter, only the number of observations varies. An overview of the reliability measures that correspond to the four personality traits is provided in Appendix Table 6.14. To ensure that the variables helplessness and hopelessness are not only dimensions or facets of one single personality trait, the correlation between the personality trait variables, the behavioral items that are used to construct the personality trait variables, the two indicators of helplessness and hopelessness as well as the five scores for mental ill-health is checked. The results are presented in Appendix Table 6.15, revealing that the correlation size is not particularly high for one single behavioral item or within one single group of behavioral items, but rather is relatively low (below $r=0.25$ ).

\subsubsection{Additional Controls}

In addition to the variables discussed above, controls are included for the individual's cognitive ability at the age of 10 and the individual family background at birth. Detailed information is provided in Chapter 3 Subsection 3.2.3.

\subsection{Results}

The results are based upon linear probability and OLS regression methods. All regressions focus on the relation between emotional stability and conscientiousness, pessimistic expectations and mental health outcomes in adolescence and adulthood. To account for the possible problem of heteroscedasticity, robust standard errors are calculated. In all regressions, it is controlled for the respondent's cognitive ability, the personality traits extraversion and agreeableness, parental socioeconomic background and region of living at birth as well as the mother's age. 


\subsubsection{Personality Traits and Pessimistic Expectations}

Table 6.1: Personality Trait Change and Help- and Hopelessness

\begin{tabular}{lllll}
\hline Pessimistic Expectations (Age 16) $\ldots$ & \multicolumn{2}{l}{ Helplessness } & \multicolumn{2}{l}{ Hopelessness } \\
\hline Emotional Stability (Age 10) & $-0.094^{* * *}(0.000)$ & $-0.078^{* * *}(0.000)$ \\
Conscientiousness (Age 10) & -0.004 & $(0.844)$ & -0.016 & $(0.431)$ \\
Emotional Stability Male (Age 10) & $0.087^{* * *}(0.009)$ & 0.019 & $(0.551)$ \\
Conscientiousness Male (Age 10) & -0.025 & $(0.391)$ & 0.035 & $(0.221)$ \\
Emotional Stability (Age 16-10) $\rangle 0.5$ & $-0.125^{* * *}(0.004)$ & -0.030 & $(0.453)$ \\
Emotional Stability (Age 16-10) $\langle-0.5$ & 0.025 & $(0.486)$ & $0.127^{* * *}(0.000)$ \\
Conscientiousness (Age 16-10) $\rangle 0.5$ & -0.031 & $(0.477)$ & -0.011 & $(0.787)$ \\
Conscientiousness (Age 16-10) $\langle-0.5$ & -0.003 & $(0.940)$ & 0.011 & $(0.749)$ \\
Emotional Stability Male (Age 16-10) $\rangle 0.5$ & $0.141^{* *}$ & $(0.030)$ & 0.025 & $(0.663)$ \\
Emotional Stability Male (Age 16-10) $\langle-0.5$ & 0.019 & $(0.753)$ & -0.054 & $(0.337)$ \\
Conscientiousness Male (Age 16-10) $\rangle 0.5$ & -0.012 & $(0.855)$ & -0.001 & $(0.981)$ \\
Conscientiousness Male (Age 16-10) $\langle-0.5$ & 0.028 & $(0.636)$ & 0.010 & $(0.856)$ \\
$M a l e$ & $-0.088^{*}$ & $(0.090)$ & 0.027 & $(0.555)$ \\
\hline$N$ & 2051 & & 2051 & \\
$R^{2}$ & 0.048 & & 0.054 & \\
\hline
\end{tabular}

Source: British Cohort Study (BCS70), own calculations.

Note: $p$-values in parentheses ${ }^{*} p<0.10,{ }^{* *} p<0.05,{ }^{* * *} p<0.01$. The OLS regressions include controls for mother's age, mother's age squared, region of living and parental socioeconomic background at birth, the personality traits agreeableness and extraversion measured at the ages of 10 and 16 as well as cognitive ability measured at the age of 10 .

Table 6.1 presents the predictive power of emotional stability and conscientiousness at the age of 10 and a change in these personality traits between the ages of 10 and 16 for pessimistic expectations at the age of 16. A personality trait increase (decrease) is considered if the particular trait increases (decreases) by 0.5 of a standard deviation between the ages of 10 and 16. Individuals who do not experience a personality trait change above or below 0.5 of a standard deviation between the ages of 10 and 16 are considered as the reference group. Appendix Table 6.9 shows that in the working sample the probability of experiencing thoughts of helplessness is on average $55 \%$, 
while the probability of having thoughts of hopelessness is on average $28 \%{ }^{16}$

The results indicate that low emotional stability predicts thoughts of helplessness particularly for girls. A 1 standard deviation higher level of emotional stability at the age of 10 is associated with a 9.4 percentage points decrease in the probability of experiencing thoughts of helplessness at the age of 16. Furthermore, an emotional stability increase by more than 0.5 of a standard deviation between the ages of 10 and 16 is significantly related to a 12.5 percentage points decrease in the probability of experiencing thoughts of helplessness at the age of 16 . The related male interaction terms suggest that there is no such relationship for boys.

The analysis shows that a 1 standard deviation higher level of emotional stability at the age of 10 is significantly associated with a 7.8 percentage points decrease in the probability of experiencing thoughts of hopelessness at the age of 16. An emotional stability decrease by more than 0.5 of a standard deviation between the ages of 10 and 16 is associated with a 12.7 percentage points increase in the probability of having thoughts of hopelessness for both girls and boys. Furthermore, it is found that boys have a 9 percentage points lower probability of experiencing thoughts of helplessness than girls. However, for thoughts of hopelessness, there are no gender differences.

These results are in line with the literature. Psychologists argue that "repeated or chronic negative life events in childhood may directly contribute to the development of a more negative cognitive style as children, over time, internalize the belief that negative events are stable, have negative consequences, and are attributable to aspects of themselves" (Mezulis et al., 2006, p. 1013). Girls are expected to be more likely to have pessimistic expectations than boys in childhood and adolescence. Studies in psychology offer multiple explanation approaches for this phenomenon (Hankin and Abramson, 2001, 2002; Hyde et al., 2008).

First, low emotional stability is expected to be positively correlated with pessimistic thoughts, because it "directly affects children's perception of the aversiveness of events" (Hyde et al., 2008, p. 298). Thus, low emotionally stable children and adolescence tend to perceive negative life events as more stressful and threatening than

\footnotetext{
16 In the working sample, the probability of experiencing thoughts of helplessness at the age of 16 is on average $56 \%$ for girls and $52 \%$ for boys. The probability of having thoughts of hopelessness at the age of 16 is on average $29 \%$ for girls and $27 \%$ for boys (see Appendix Tables 6.7 and 6.8).
} 
more emotionally stable ones and are more likely to develop pessimistic thoughts and expectations. Girls might have a higher appraisal of negative life events due to lower average emotional stability.

Second, gender differences regarding pessimistic expectations might arise related to differences in gender socialization. Hankin and Abramson (2001) argue that girls might encode negative life events in more detail than boys, because parents tend to discuss emotional issues such as sadness more elaborately with their daughters compared with their sons. Furthermore, mothers tend to be more controlling to their daughters compared with their sons, which is related to a rise in negative self-evaluations and the tendency to take responsibility for failure (p. 785).

Third, pessimistic thoughts and expectations are related to the actual number of negative life events. "Girls overall experience somewhat more negative life events than boys do [...] and are considerably more likely to experience child sexual abuse. Girls also experience more interpersonal negative events" (Hyde et al., 2008, p. 305). Furthermore, females seem to be more critical regarding their physical appearance and "numerous studies have found that girls and women have more negative body esteem than men and boys" (Hyde et al., 2008, p. 297). However, controlling for the source, number and severity of negative life events would exceed the scope of the dissertation.

\subsubsection{Predictive Power of Pessimistic Expectations}

Subsequently, the predictive power of pessimistic expectations at the age of 16 for mental health problems at the ages of 16, 26, 34 and 42 is addressed. ${ }^{17}$ Thereby, the dependent variable mental ill-health at the ages of 16, 26, 34 and 42 is stepwise regressed on personality traits as well as pessimistic expectations measured at the age of 16 . The focus lies again on personality types comprising (1) more emotionally stable and more conscientious, (2) more emotionally stable and less conscientious, (3) less emotionally stable and more conscientious and (4) less emotionally stable and less conscientious individuals are built. The reference group comprises more emotionally stable and more conscientious individuals, i.e. theoretically considered as

\footnotetext{
17 Mental ill-health at the age of 30 is also analyzed. These coefficients are qualitatively similar to those at the age of 34 . However, the results are omitted from the tables for the sake of readability, but are available upon request.
} 
the most favorable combination. Pessimistic expectations are measured by a dummy for having or not having thoughts of helplessness or hopelessness. The results are presented in Tables 6.2 and 6.3.

Table 6.2 shows that scoring low in both emotional stability and conscientiousness at the age of 16 is significantly positively associated with mental health problems at adolescence and adult age. Being less emotionally stable but scoring higher in conscientiousness seems to be able to mitigate the negative relation between emotional stability and mental health problems. ${ }^{18}$ After including the variable thoughts of helplessness at the age of 16 into the regression, the coefficients of the personality types including low emotional stability slightly decrease. This indicates that pessimistic expectations partially mediate the relation between low emotional stability and mental health problems which is in line with the theoretical considerations.

The results yield that thoughts of helplessness significantly predict mental ill-health after controlling for personality. This supports the argumentation that pessimistic expectations are not only facets of personality traits. For women, experiencing thoughts of helplessness at the age of 16 is significantly positively related with mental ill-health in adolescence by 0.7 of a standard deviation. The size of the helplessness coefficients is lower at adult age, but remains highly significant with around 0.3 of a standard deviation. For men, the positive relation between helplessness and mental ill-health is significantly smaller at the ages of 16 and 26 by around 0.22 of a standard deviation. The results for thoughts of hopelessness are similar, although the size of the coefficient is smaller, between 0.12 and 0.19 of a standard deviation. At the age of 16 , there is no significant relation between thoughts of hopelessness and mental ill-health for men.

However, one might argue that mental health problems at both adolescent and adult age are to a major extent related to a genetic predisposition, rather than differences in expectations and the related behavior. Hence, personality and pessimistic expectations at the age of 16 would only reflect mental ill-health at the age of 16 in the regressions for the older ages. Therefore, a third column is added at the ages of 26, 34 and 42 where it is controlled for mental health at the age of 16 . Table 6.2 shows that a one-point increase in the malaise score at the age of 16 is associated

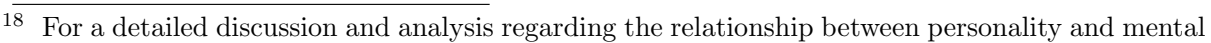
ill-health, see Chapter 5.
} 


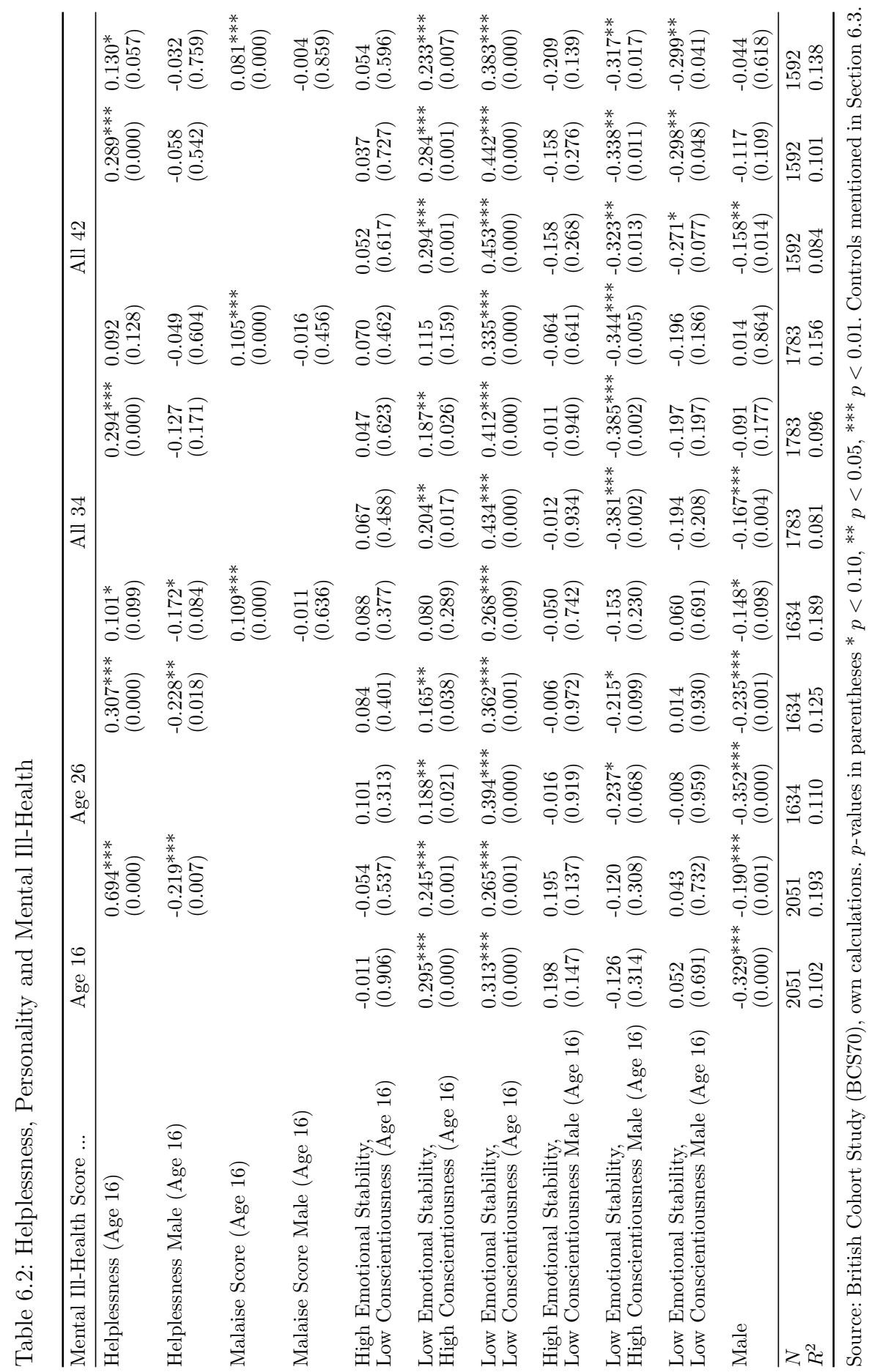




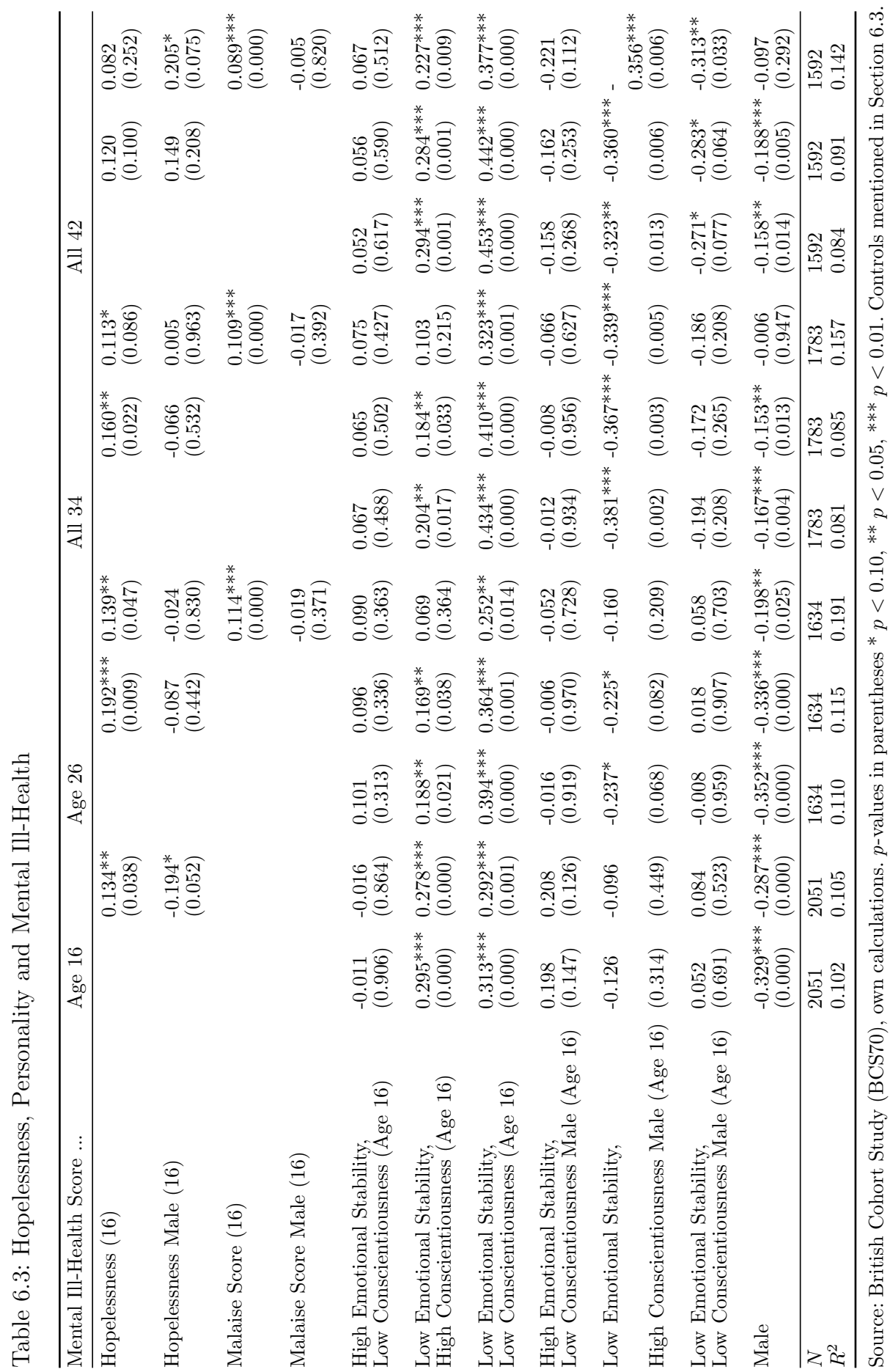


with a mental ill-health increase of around 0.1 of a standard deviation at adult age. This result is in line with the literature and suggests that mental health problems are likely to recur throughout life (Johnston et al., 2013). Controlling for mental health problems at the age of 16 reduces the size and significance of the coefficients for helplessness. Nevertheless, having pessimistic expectations at the age of 16 is still (borderline) significantly positive associated with mental health problems by around 0.1 of a standard deviation. This is comparable with the influence of mental ill-health at the age of 16 and supports the argumentation that beside an unquestionable underlying genetical component, behavioral variation related to pessimistic expectations might also matter for mental health outcomes.

In addition, it is interesting to know whether the predictive power of pessimistic expectations for mental health problems varies with personality characteristics, particularly for the high-risk group that comprises low emotionally stable and low conscientious individuals. To test this, the dummy for having or not having thoughts of helplessness (hopelessness) is interacted with the emotional stability and conscientiousness interaction terms introduced above. The reference group comprises low emotionally stable and low conscientious individuals who have thoughts of helplessness.

Table 6.4: Interaction between Helplessness and Personality

\begin{tabular}{|c|c|c|c|c|c|}
\hline Mental Ill-Health Score ... & Age 16 & Age 26 & & All 34 & All 42 \\
\hline $\begin{array}{l}\text { High Emotional Stability, } \\
\text { High Conscientiousness } \\
\text { Help (Age 16) }\end{array}$ & $\begin{array}{l}-0.925^{* * *} \\
(0.000)\end{array}$ & $\begin{array}{l}-0.625^{* * * *} \\
(0.000)\end{array}$ & $\begin{array}{c}{ }^{*}-0.333^{* *} \\
(0.012)\end{array}$ & $\begin{array}{l}-0.699^{* * *}-0.456^{* * *} \\
(0.000) \quad(0.001)\end{array}$ & $\begin{array}{c}-0.776^{* * *}-0.576^{* * *} \\
(0.000) \quad(0.000)\end{array}$ \\
\hline $\begin{array}{l}\text { High Emotional Stability, } \\
\text { High Conscientiousness } \\
\text { Helpless (Age 16) }\end{array}$ & $\begin{array}{l}-0.345^{* * *} \\
(0.003)\end{array}$ & $\begin{array}{l}-0.344^{* *} \\
(0.013)\end{array}$ & $\begin{array}{l}-0.206 \\
(0.118)\end{array}$ & $\begin{array}{l}-0.508^{* * *}-0.425^{* * *} \\
(0.000) \quad(0.002)\end{array}$ & $\begin{array}{l}-0.567^{* * *}-0.494^{* * *} \\
(0.000) \quad(0.001)\end{array}$ \\
\hline $\begin{array}{l}\text { High Emotional Stability, } \\
\text { Low Conscientiousness } \\
\text { Help (Age 16) }\end{array}$ & $\begin{array}{l}-1.035^{* * *} \\
(0.000)\end{array}$ & $\begin{array}{l}-0.491^{* * * *} \\
(0.007)\end{array}$ & $\begin{array}{l}-0.170 \\
(0.329)\end{array}$ & $\begin{array}{l}-0.619^{* * *}-0.326^{* *} \\
(0.000) \quad(0.044)\end{array}$ & $\begin{array}{ll}-0.583^{* * *}-0.357^{*} \\
(0.004) & (0.067)\end{array}$ \\
\hline $\begin{array}{l}\text { High Emotional Stability, } \\
\text { Low Conscientiousness } \\
\text { Helpless (Age 16) }\end{array}$ & $\begin{array}{l}-0.348^{* *} \\
(0.023)\end{array}$ & $\begin{array}{l}-0.297^{*} \\
(0.073)\end{array}$ & $\begin{array}{l}-0.178 \\
(0.284)\end{array}$ & $\begin{array}{l}-0.480^{* * *}-0.396 * * \\
(0.008) \quad(0.022)\end{array}$ & $\begin{array}{l}-0.624^{* * *}-0.541^{* * *} \\
(0.000) \quad(0.002)\end{array}$ \\
\hline $\begin{array}{l}\text { Low Emotional Stability, } \\
\text { High Conscientiousness } \\
\text { Help (Age 16) }\end{array}$ & $\begin{array}{l}-0.894^{* * *} \\
(0.000)\end{array}$ & $\begin{array}{l}-0.583^{* * * *} \\
(0.000)\end{array}$ & $\begin{array}{l}-0.305^{* *} \\
(0.024)\end{array}$ & $\begin{array}{l}-0.691^{* * *}-0.453^{* * *} \\
(0.000) \quad(0.003)\end{array}$ & $\begin{array}{c}-0.628^{* * *}-0.421^{* *} \\
(0.000) \quad(0.013)\end{array}$ \\
\hline Low Emotional Stability, & 0.050 & -0.092 & -0.089 & $\begin{array}{l}-0.227 \\
\quad \text { Contin }\end{array}$ & $\begin{array}{cc}-0.177 & -0.192 \\
\text { nued on } & \text { next page }\end{array}$ \\
\hline
\end{tabular}


... Table 6.4 continued

\begin{tabular}{|c|c|c|c|c|c|c|c|}
\hline Mental Ill-Health Score ... & Age 16 & Age 26 & & All 34 & & All 42 & \\
\hline $\begin{array}{l}\text { High Conscientiousness } \\
\text { Helpless (Age 16) }\end{array}$ & $(0.702)$ & $(0.534)$ & $(0.520)$ & $(0.201)$ & $(0.130)$ & $(0.275)$ & $(0.231)$ \\
\hline $\begin{array}{l}\text { Low Emotional Stability, } \\
\text { Low Conscientiousness } \\
\text { Help (Age 16) }\end{array}$ & $\begin{array}{l}-0.756^{* * *} \\
(0.000)\end{array}$ & $\begin{array}{l}-0.236 \\
(0.207)\end{array}$ & $\begin{array}{l}0.024 \\
(0.893)\end{array}$ & $\begin{array}{l}-0.487 * * * \\
(0.004)\end{array}$ & $\begin{array}{l}-0.293^{*} \\
(0.070)\end{array}$ & $\begin{array}{l}-0.558^{* * *} \\
(0.002)\end{array}$ & $\begin{array}{l}-0.390 * * \\
(0.026)\end{array}$ \\
\hline $\begin{array}{l}\text { High Emotional Stability, } \\
\text { High Conscientiousness } \\
\text { Help Male (Age 16) }\end{array}$ & $\begin{array}{l}0.191 \\
(0.274)\end{array}$ & $\begin{array}{l}0.081 \\
(0.710)\end{array}$ & $\begin{array}{l}-0.000 \\
(0.999)\end{array}$ & $\begin{array}{l}0.151 \\
(0.475)\end{array}$ & $\begin{array}{l}0.125 \\
(0.539)\end{array}$ & $\begin{array}{l}0.226 \\
(0.294)\end{array}$ & $\begin{array}{l}0.230 \\
(0.278)\end{array}$ \\
\hline $\begin{array}{l}\text { High Emotional Stability, } \\
\text { High Conscientiousness } \\
\text { Helpless Male (Age 16) }\end{array}$ & $\begin{array}{l}0.109 \\
(0.552)\end{array}$ & $\begin{array}{l}-0.014 \\
(0.950)\end{array}$ & $\begin{array}{l}-0.098 \\
(0.637)\end{array}$ & $\begin{array}{l}0.142 \\
(0.516)\end{array}$ & $\begin{array}{l}0.132 \\
(0.533)\end{array}$ & $\begin{array}{l}0.253 \\
(0.256)\end{array}$ & $\begin{array}{l}0.259 \\
(0.236)\end{array}$ \\
\hline $\begin{array}{l}\text { High Emotional Stability, } \\
\text { Low Conscientiousness } \\
\text { Help Male (Age 16) }\end{array}$ & $\begin{array}{l}0.491^{* *} \\
(0.031)\end{array}$ & $\begin{array}{l}0.165 \\
(0.583)\end{array}$ & $\begin{array}{l}-0.005 \\
(0.985)\end{array}$ & $\begin{array}{l}0.249 \\
(0.379)\end{array}$ & $\begin{array}{l}0.120 \\
(0.654)\end{array}$ & $\begin{array}{l}0.028 \\
(0.919)\end{array}$ & $\begin{array}{l}-0.023 \\
(0.932)\end{array}$ \\
\hline $\begin{array}{l}\text { High Emotional Stability, } \\
\text { Low Conscientiousness } \\
\text { Helpless Male (Age 16) }\end{array}$ & $\begin{array}{l}0.207 \\
(0.366)\end{array}$ & $\begin{array}{l}-0.101 \\
(0.692)\end{array}$ & $\begin{array}{l}-0.198 \\
(0.425)\end{array}$ & $\begin{array}{l}0.086 \\
(0.731)\end{array}$ & $\begin{array}{l}0.047 \\
(0.843)\end{array}$ & $\begin{array}{l}0.139 \\
(0.583)\end{array}$ & $\begin{array}{l}0.085 \\
(0.731)\end{array}$ \\
\hline $\begin{array}{l}\text { Low Emotional Stability, } \\
\text { High Conscientiousness } \\
\text { Help Male (Age 16) }\end{array}$ & $\begin{array}{l}0.187 \\
(0.371)\end{array}$ & $\begin{array}{l}0.251 \\
(0.316)\end{array}$ & $\begin{array}{l}0.195 \\
(0.417)\end{array}$ & $\begin{array}{l}0.089 \\
(0.726)\end{array}$ & $\begin{array}{l}0.068 \\
(0.783)\end{array}$ & $\begin{array}{l}0.241 \\
(0.373)\end{array}$ & $\begin{array}{l}0.231 \\
(0.386)\end{array}$ \\
\hline $\begin{array}{l}\text { Low Emotional Stability, } \\
\text { High Conscientiousness } \\
\text { Helpless Male (Age 16) }\end{array}$ & $\begin{array}{l}-0.100 \\
(0.635)\end{array}$ & $\begin{array}{l}-0.520^{* *} \\
(0.031)\end{array}$ & $\begin{array}{l}-0.524^{* *} \\
(0.021)\end{array}$ & $\begin{array}{l}-0.396^{*} \\
(0.097)\end{array}$ & $\begin{array}{l}-0.362 \\
(0.112)\end{array}$ & $\begin{array}{l}-0.279 \\
(0.236)\end{array}$ & $\begin{array}{l}-0.244 \\
(0.291)\end{array}$ \\
\hline $\begin{array}{l}\text { Low Emotional Stability, } \\
\text { Low Conscientiousness } \\
\text { Help Male (Age 16) }\end{array}$ & $\begin{array}{l}0.426^{*} \\
(0.064)\end{array}$ & $\begin{array}{l}0.087 \\
(0.769)\end{array}$ & $\begin{array}{l}-0.015 \\
(0.959)\end{array}$ & $\begin{array}{l}0.087 \\
(0.743)\end{array}$ & $\begin{array}{l}-0.026 \\
(0.921)\end{array}$ & $\begin{array}{l}0.018 \\
(0.940)\end{array}$ & $\begin{array}{l}-0.010 \\
(0.965)\end{array}$ \\
\hline $\begin{array}{l}\text { Malaise Score } \\
\text { (Age 16) }\end{array}$ & & & $\begin{array}{l}0.105^{* * *} \\
(0.000)\end{array}$ & & $\begin{array}{l}0.099 * * * \\
(0.000)\end{array}$ & & $\begin{array}{l}0.079 \text { *** } \\
(0.000)\end{array}$ \\
\hline Male & $\begin{array}{l}-0.449 * * * \\
(0.004)\end{array}$ & $\begin{array}{l}-0.385^{* *} \\
(0.042)\end{array}$ & $\begin{array}{l}-0.227 \\
(0.202)\end{array}$ & $\begin{array}{c}-0.330^{*} \\
(0.086)\end{array}$ & $\begin{array}{l}-0.219 \\
(0.235)\end{array}$ & $\begin{array}{l}-0.409^{* *} \\
(0.033)\end{array}$ & $\begin{array}{c}-0.337^{*} \\
(0.073)\end{array}$ \\
\hline $\begin{array}{l}N \\
R^{2}\end{array}$ & $\begin{array}{l}2051 \\
0.197\end{array}$ & $\begin{array}{l}1634 \\
0.129\end{array}$ & $\begin{array}{l}1634 \\
0.193\end{array}$ & $\begin{array}{l}1783 \\
0.103\end{array}$ & $\begin{array}{l}1783 \\
0.161\end{array}$ & $\begin{array}{l}1592 \\
0.113\end{array}$ & $\begin{array}{l}1592 \\
0.150\end{array}$ \\
\hline
\end{tabular}

Source: British Cohort Study (BCS70), own calculations.

Note: $p$-values in parentheses ${ }^{*} p<0.10,{ }^{* *} p<0.05,{ }^{* * *} p<0.01$. The OLS regressions include controls for mother's age, mother's age squared, region of living and parental socioeconomic background at birth, the personality traits agreeableness and extraversion measured at the age of 16 as well as cognitive ability measured at the age of 10 . 
Table 6.4 reveals that low emotionally stable and low conscientious individuals who have the highest risk of mental health problems - are (borderline) significantly less likely to experience mental health problems at adolescence and adult age if they do not have thoughts of helplessness at the age of 16 . The results further show that the risk of suffering from mental health problems is significantly smaller for less conscientious individuals who have thoughts of helplessness, but who are more emotionally stable compared with those who are less emotionally stable, which might be related to differences in the individual stress-reactivity. This result suggests that already targeting pessimistic expectations and maladaptive coping behaviors in school might help to reduce mental ill-health particularly among the high-risk individuals.

In terms of gender differences, Table 6.2 suggests that men are less likely to experience mental health problems by between 0.16 and 0.33 of a standard deviation compared to women. This gender gap decreases with age. After controlling for thoughts of helplessness, the male coefficient substantially decreases in size and significance. It is further found that the association between thoughts of helplessness and mental health problems is significantly stronger for women than men in adolescence and young adult age. Table 6.4 further shows that low emotionally stable but more conscientious men who have thoughts of helplessness are (borderline) significantly less likely to experience mental health problems in adulthood compared with women who have the same characteristics. This suggests that low emotionally stable women are particularly vulnerable regarding thoughts of helplessness, while low emotionally stable, but more conscientious men are more likely to overcome these pessimistic expectations. However, for thoughts of hopelessness, there are no gender differences (Table 6.5).

These results are in line with the psychological literature and the theoretical model showing that pessimistic expectations are significantly positively associated with depressive symptoms (Hankin and Abramson, 2001, 2002; Hyde et al., 2008). Furthermore, girls seem to be more sensitive to pessimistic expectations compared to boys, as also found in previous studies. Looking at the latter results, one explanation approach provided by psychologists is that girls and women tend to ruminate more than boys and men, which is considered as another type of cognitive vulnerability to stress (Hankin and Abramson, 2001; Hyde et al., 2008; Rood et al., 2012). Thus, the possibility of both more negative life events in adolescence for girls (see discussion 
in the previous subsection) and the higher sensitivity to pessimistic expectations related to rumination might at least partly explain gender differences in depressive symptoms.

Table 6.5: Interaction between Hopelessness and Personality

\begin{tabular}{|c|c|c|c|c|c|c|c|}
\hline \multirow{2}{*}{$\begin{array}{l}\text { Mental Ill-Health Score ... } \\
\text { High Emotional Stability, } \\
\text { High Conscientiousness } \\
\text { Hope (Age 16) }\end{array}$} & \multirow{2}{*}{$\begin{array}{l}\text { Age } 16 \\
-0.464^{* * *} \\
(0.000)\end{array}$} & \multicolumn{2}{|l|}{ Age 26} & \multicolumn{2}{|l|}{ All 34} & \multicolumn{2}{|l|}{ All 42} \\
\hline & & $\begin{array}{l}-0.647^{* *} \\
(0.000)\end{array}$ & $\begin{array}{l}-0.487^{* * *} \\
(0.002)\end{array}$ & $\begin{array}{c}-0.747^{* *} \\
(0.000)\end{array}$ & $\begin{array}{l}-0.622^{* * * *} \\
(0.000)\end{array}$ & $\begin{array}{l}-0.645^{* * *} \\
(0.000)\end{array}$ & $\begin{array}{c}* 0.539^{* * *} \\
(0.001)\end{array}$ \\
\hline $\begin{array}{l}\text { High Emotional Stability, } \\
\text { High Conscientiousness } \\
\text { Hopeless (Age 16) }\end{array}$ & $\begin{array}{l}-0.342^{* *} \\
(0.014)\end{array}$ & $\begin{array}{l}-0.590^{* * *} \\
(0.001)\end{array}$ & $\begin{array}{l}-0.475^{\text {*** }} \\
(0.005)\end{array}$ & $\begin{array}{l}-0.725^{* * *} \\
(0.000)\end{array}$ & $\begin{array}{l}-0.645^{* * * *} \\
(0.000)\end{array}$ & $\begin{array}{l}-0.505^{* * *} \\
(0.005)\end{array}$ & $\begin{array}{c}* 0.437^{* *} \\
(0.012)\end{array}$ \\
\hline $\begin{array}{l}\text { High Emotional Stability, } \\
\text { Low Conscientiousness } \\
\text { Hope (Age 16) }\end{array}$ & $\begin{array}{l}-0.512^{* * *} \\
(0.000)\end{array}$ & $\begin{array}{l}-0.590^{* * *} \\
(0.001)\end{array}$ & $\begin{array}{l}-0.431^{* *} \\
(0.011)\end{array}$ & $\begin{array}{l}-0.699^{* *} \\
(0.000)\end{array}$ & $\begin{array}{l}-0.555^{* * * *} \\
(0.001)\end{array}$ & $\begin{array}{l}-0.544^{* * *} \\
(0.003)\end{array}$ & $\begin{array}{c}{ }^{*}-0.421^{* *} \\
(0.018)\end{array}$ \\
\hline $\begin{array}{l}\text { High Emotional Stability, } \\
\text { Low Conscientiousness } \\
\text { Hopeless (Age 16) }\end{array}$ & $\begin{array}{l}-0.271 \\
(0.225)\end{array}$ & $\begin{array}{l}-0.357 \\
(0.165)\end{array}$ & $\begin{array}{l}-0.256 \\
(0.310)\end{array}$ & $\begin{array}{l}-0.609^{* *} \\
(0.007)\end{array}$ & $\begin{array}{l}-0.537^{* *} \\
(0.014)\end{array}$ & $\begin{array}{l}-0.593^{* *} \\
(0.018)\end{array}$ & $\begin{array}{c}-0.526^{* *} \\
(0.032)\end{array}$ \\
\hline $\begin{array}{l}\text { Low Emotional Stability, } \\
\text { High Conscientiousness } \\
\text { Hope (Age 16) }\end{array}$ & $\begin{array}{l}-0.159 \\
(0.251)\end{array}$ & $\begin{array}{l}-0.523^{* *} \\
(0.002)\end{array}$ & $\begin{array}{l}-0.458^{* * *} \\
(0.003)\end{array}$ & $\begin{array}{l}-0.569^{* * *} \\
(0.001)\end{array}$ & $\begin{array}{l}-0.526^{* * * *} \\
(0.001)\end{array}$ & $\begin{array}{c}-0.304^{*} \\
(0.088)\end{array}$ & $\begin{array}{c}-0.262 \\
(0.133)\end{array}$ \\
\hline $\begin{array}{l}\text { Low Emotional Stability, } \\
\text { High Conscientiousness } \\
\text { Hopeless (Age 16) }\end{array}$ & $\begin{array}{c}-0.112 \\
(0.479)\end{array}$ & $\begin{array}{l}-0.285 \\
(0.141)\end{array}$ & $\begin{array}{l}-0.257 \\
(0.148)\end{array}$ & $\begin{array}{l}-0.489^{* *} \\
(0.007)\end{array}$ & $\begin{array}{l}-0.462^{* * * *} \\
(0.005)\end{array}$ & $\begin{array}{c}-0.333^{*} \\
(0.070)\end{array}$ & $\begin{array}{c}-0.299^{*} \\
(0.097)\end{array}$ \\
\hline $\begin{array}{l}\text { Low Emotional Stability, } \\
\text { Low Conscientiousness } \\
\text { Hope (Age 16) }\end{array}$ & $\begin{array}{l}-0.208 \\
(0.171)\end{array}$ & $\begin{array}{l}-0.407^{* *} \\
(0.024)\end{array}$ & $\begin{array}{l}-0.339^{* *} \\
(0.046)\end{array}$ & $\begin{array}{l}-0.573^{* *} \\
(0.001)\end{array}$ & $\begin{array}{l}-0.519 \text { **** } \\
(0.002)\end{array}$ & $\begin{array}{c}-0.321^{*} \\
(0.089)\end{array}$ & $\begin{array}{c}-0.267 \\
(0.142)\end{array}$ \\
\hline $\begin{array}{l}\text { High Emotional Stability, } \\
\text { High Conscientiousness } \\
\text { Hope Male (Age 16) }\end{array}$ & $\begin{array}{l}0.183 \\
(0.376)\end{array}$ & $\begin{array}{l}0.384^{*} \\
(0.096)\end{array}$ & $\begin{array}{l}0.282 \\
(0.207)\end{array}$ & $\begin{array}{l}0.274 \\
(0.272)\end{array}$ & $\begin{array}{l}0.239 \\
(0.317)\end{array}$ & $\begin{array}{l}0.139 \\
(0.605)\end{array}$ & $\begin{array}{l}0.137 \\
(0.605)\end{array}$ \\
\hline $\begin{array}{l}\text { High Emotional Stability, } \\
\text { High Conscientiousness } \\
\text { Hopeless Male (Age 16) }\end{array}$ & $\begin{array}{l}-0.010 \\
(0.964)\end{array}$ & $\begin{array}{l}0.588^{* *} \\
(0.022)\end{array}$ & $\begin{array}{l}0.531^{* *} \\
(0.034)\end{array}$ & $\begin{array}{l}0.331 \\
(0.221)\end{array}$ & $\begin{array}{l}0.368 \\
(0.157)\end{array}$ & $\begin{array}{l}0.170 \\
(0.567)\end{array}$ & $\begin{array}{l}0.218 \\
(0.454)\end{array}$ \\
\hline $\begin{array}{l}\text { High Emotional Stability, } \\
\text { Low Conscientiousness } \\
\text { Hope Male (Age 16) }\end{array}$ & $\begin{array}{l}0.471^{* *} \\
(0.047)\end{array}$ & $\begin{array}{l}0.509^{*} \\
(0.053)\end{array}$ & $\begin{array}{l}0.328 \\
(0.202)\end{array}$ & $\begin{array}{l}0.379 \\
(0.174)\end{array}$ & $\begin{array}{l}0.256 \\
(0.339)\end{array}$ & $\begin{array}{l}-0.072 \\
(0.801)\end{array}$ & $\begin{array}{l}-0.164 \\
(0.555)\end{array}$ \\
\hline $\begin{array}{l}\text { High Emotional Stability, } \\
\text { Low Conscientiousness } \\
\text { Hopeless Male (Age 16) }\end{array}$ & $\begin{array}{l}-0.018 \\
(0.955)\end{array}$ & $\begin{array}{l}0.176 \\
(0.623)\end{array}$ & $\begin{array}{l}0.133 \\
(0.709)\end{array}$ & $\begin{array}{l}0.095 \\
(0.765)\end{array}$ & $\begin{array}{l}0.114 \\
(0.708)\end{array}$ & $\begin{array}{l}0.298 \\
(0.460)\end{array}$ & $\begin{array}{l}0.359 \\
(0.354)\end{array}$ \\
\hline Low Emotional Stability, & -0.037 & 0.150 & 0.121 & -0.144 & $\begin{array}{l}-0.140 \\
\quad \text { Contin }\end{array}$ & $\begin{array}{l}-0.372 \\
\text { nued on } n\end{array}$ & $\begin{array}{c}-0.362 \\
\text { ext page... }\end{array}$ \\
\hline
\end{tabular}


... Table 6.5 continued

\begin{tabular}{|c|c|c|c|c|c|c|c|}
\hline Mental Ill-Health Score ... & Age 16 & Age 26 & & All 34 & & All 42 & \\
\hline $\begin{array}{l}\text { High Conscientiousness } \\
\text { Hope Male (Age 16) }\end{array}$ & $(0.873)$ & $(0.544)$ & $(0.618)$ & $(0.584)$ & $(0.584)$ & $(0.189)$ & $(0.195)$ \\
\hline $\begin{array}{l}\text { Low Emotional Stability, } \\
\text { High Conscientiousness } \\
\text { Hopeless Male (Age 16) }\end{array}$ & $\begin{array}{l}0.108 \\
(0.673)\end{array}$ & $\begin{array}{l}0.271 \\
(0.349)\end{array}$ & $\begin{array}{l}0.230 \\
(0.423)\end{array}$ & $\begin{array}{l}0.095 \\
(0.742)\end{array}$ & $\begin{array}{l}0.074 \\
(0.792)\end{array}$ & $\begin{array}{l}0.186 \\
(0.555)\end{array}$ & $\begin{array}{l}0.193 \\
(0.531)\end{array}$ \\
\hline $\begin{array}{l}\text { Low Emotional Stability, } \\
\text { Low Conscientiousness } \\
\text { Hope Male (Age 16) }\end{array}$ & $\begin{array}{l}0.318 \\
(0.189)\end{array}$ & $\begin{array}{l}0.749^{* * * *} \\
(0.007)\end{array}$ & $\begin{array}{l}0.637 * * \\
(0.018)\end{array}$ & $\begin{array}{l}0.376 \\
(0.186)\end{array}$ & $\begin{array}{l}0.278 \\
(0.308)\end{array}$ & $\begin{array}{l}0.024 \\
(0.935)\end{array}$ & $\begin{array}{l}-0.028 \\
(0.922)\end{array}$ \\
\hline $\begin{array}{l}\text { Malaise Score } \\
\text { (Age 16) }\end{array}$ & & & $\begin{array}{l}0.106^{* * *} \\
(0.000)\end{array}$ & & $\begin{array}{l}0.102^{* * *} \\
(0.000)\end{array}$ & & $\begin{array}{l}0.087 * * * \\
(0.000)\end{array}$ \\
\hline Male & $\begin{array}{l}-0.471^{* *} \\
(0.012)\end{array}$ & $\begin{array}{l}-0.792^{* * *} \\
(0.000)\end{array}$ & $\begin{array}{l}-0.615^{* * * *} \\
(0.003)\end{array}$ & $\begin{array}{l}-0.480^{* *} \\
(0.038)\end{array}$ & $\begin{array}{l}-0.354 \\
(0.112)\end{array}$ & $\begin{array}{l}-0.330 \\
(0.191)\end{array}$ & $\begin{array}{l}-0.247 \\
(0.318)\end{array}$ \\
\hline$N$ & 2051 & 1634 & 1634 & 1783 & 1783 & 1592 & 1592 \\
\hline$R^{2}$ & 0.106 & 0.123 & 0.196 & 0.094 & 0.165 & 0.099 & 0.150 \\
\hline
\end{tabular}

Source: British Cohort Study (BCS70), own calculations.

Note: $p$-values in parentheses ${ }^{*} p<0.10,{ }^{* *} p<0.05,{ }^{* * *} p<0.01$. The OLS regressions include controls for mother's age, mother's age squared, region of living and parental socioeconomic background at birth, the personality traits agreeableness and extraversion measured at the age of 16 as well as cognitive ability measured at the age of 10 .

\subsubsection{Change in Pessimistic Expectations}

Finally, the predictive power of a change in pessimistic expectations between the ages of 16 and 30 for mental health outcomes at the ages of 30, 34 and 42 is analyzed. The psychological literature argues that states (e.g., pessimistic expectations) are more volatile than personality traits, whereby negative thought patterns might change throughout life due to various environmental influences (Roberts, 2009). The results in the previous subsection have shown that the size and significance of the coefficients for the variables helplessness and hopelessness variables decrease with age which might be dedicated to such changes in pessimistic expectations. The analysis now emphasizes this issue by estimating the association between a change in pessimistic expectations between the ages of 16 and 30 and mental health problems at the ages of 30, 34 and 42. Helplessness is measured at the ages of 16 and 30, which is exploited to gain an idea about the predictive power of a change in pessimistic expectations for mental health problems. Unfortunately, it is impossible to perform the same 
empirical test for thoughts of hopelessness, because the survey does not provide a comparable measure for hopelessness at older age.

Table 6.6: Helplessness Change, Gender and Mental Ill-Health

\begin{tabular}{|c|c|c|c|c|c|c|}
\hline Mental Ill-Health Score ... & Age 30 & & Age 34 & & All 42 & \\
\hline $\begin{array}{l}\text { Helplessness } \\
\text { (Age 16) }\end{array}$ & $\begin{array}{l}0.435 * * * \\
(0.000)\end{array}$ & $\begin{array}{l}0.330^{* * *} \\
(0.000)\end{array}$ & $\begin{array}{l}0.354^{* * *} \\
(0.000)\end{array}$ & $\begin{array}{l}0.250 * * * \\
(0.000)\end{array}$ & $\begin{array}{l}0.275^{* * *} \\
(0.000)\end{array}$ & $\begin{array}{l}0.184^{* * *} \\
(0.000)\end{array}$ \\
\hline $\begin{array}{l}\text { Helplessness Change } \\
(\text { Age } 30-16)>1\end{array}$ & $\begin{array}{l}0.588^{* * * *} \\
(0.000)\end{array}$ & $\begin{array}{l}0.547^{* * *} \\
(0.000)\end{array}$ & $\begin{array}{l}0.356^{* * *} \\
(0.000)\end{array}$ & $\begin{array}{l}0.319^{* * *} \\
(0.000)\end{array}$ & $\begin{array}{l}0.234^{* * *} \\
(0.003)\end{array}$ & $\begin{array}{l}0.205^{* * *} \\
(0.007)\end{array}$ \\
\hline $\begin{array}{l}\text { Helplessness Change } \\
\text { (Age 30-16) }<-1\end{array}$ & $\begin{array}{l}-0.553^{* * *} \\
(0.000)\end{array}$ & $\begin{array}{l}-0.524^{* * *} \\
(0.000)\end{array}$ & $\begin{array}{l}-0.406^{* * *} \\
(0.000)\end{array}$ & $\begin{array}{l}-0.379^{* * *} \\
(0.000)\end{array}$ & $\begin{array}{l}-0.316^{* * *} \\
(0.000)\end{array}$ & $\begin{array}{l}-0.276^{* * *} \\
(0.001)\end{array}$ \\
\hline $\begin{array}{l}\text { Helplessness Change } \\
\text { Male (Age } 30-16)\rangle 1\end{array}$ & $\begin{array}{l}0.096 \\
(0.354)\end{array}$ & $\begin{array}{l}0.065 \\
(0.521)\end{array}$ & $\begin{array}{l}-0.047 \\
(0.675)\end{array}$ & $\begin{array}{l}-0.069 \\
(0.532)\end{array}$ & $\begin{array}{l}0.087 \\
(0.471)\end{array}$ & $\begin{array}{l}0.071 \\
(0.548)\end{array}$ \\
\hline $\begin{array}{l}\text { Helplessness Change } \\
\text { Male (Age 30-16) }<-1\end{array}$ & $\begin{array}{l}-0.082 \\
(0.350)\end{array}$ & $\begin{array}{l}-0.041 \\
(0.633)\end{array}$ & $\begin{array}{l}-0.194^{*} \\
(0.059)\end{array}$ & $\begin{array}{l}-0.137 \\
(0.172)\end{array}$ & $\begin{array}{l}-0.106 \\
(0.335)\end{array}$ & $\begin{array}{l}-0.077 \\
(0.481)\end{array}$ \\
\hline $\begin{array}{l}\text { Emotional Stability } \\
\text { (Age 16) }\end{array}$ & $\begin{array}{l}-0.115^{* * *} \\
(0.000)\end{array}$ & $\begin{array}{l}-0.085^{* * *} \\
(0.001)\end{array}$ & $\begin{array}{l}-0.102^{* * *} \\
(0.001)\end{array}$ & $\begin{array}{l}-0.072^{* *} \\
(0.015)\end{array}$ & $\begin{array}{l}-0.169^{* * *} \\
(0.000)\end{array}$ & $\begin{array}{l}-0.144^{* * *} \\
(0.000)\end{array}$ \\
\hline $\begin{array}{l}\text { Conscientiousness } \\
\text { (Age 16) }\end{array}$ & $\begin{array}{l}-0.033 \\
(0.185)\end{array}$ & $\begin{array}{l}-0.027 \\
(0.264)\end{array}$ & $\begin{array}{l}-0.076^{* *} \\
(0.012)\end{array}$ & $\begin{array}{l}-0.070^{* *} \\
(0.016)\end{array}$ & $\begin{array}{l}-0.003 \\
(0.920)\end{array}$ & $\begin{array}{l}0.003 \\
(0.903)\end{array}$ \\
\hline $\begin{array}{l}\text { Malaise Score } \\
\text { (Age 16) }\end{array}$ & & $\begin{array}{l}0.082^{* * *} \\
(0.000)\end{array}$ & & $\begin{array}{l}0.081^{* * *} \\
(0.000)\end{array}$ & & $\begin{array}{l}0.067^{* * *} \\
(0.000)\end{array}$ \\
\hline Male & $\begin{array}{l}-0.276^{* * *} \\
(0.000)\end{array}$ & $\begin{array}{l}-0.214^{* * *} \\
(0.000)\end{array}$ & $\begin{array}{l}-0.181^{* * *} \\
(0.008)\end{array}$ & $\begin{array}{l}-0.124^{*} \\
(0.067)\end{array}$ & $\begin{array}{l}-0.247^{* * *} \\
(0.000)\end{array}$ & $\begin{array}{l}-0.198^{* * *} \\
(0.004)\end{array}$ \\
\hline$N$ & 2050 & 2050 & 1783 & 1783 & 1592 & 1592 \\
\hline$R^{2}$ & 0.220 & 0.259 & 0.160 & 0.197 & 0.136 & 0.160 \\
\hline
\end{tabular}

Source: British Cohort Study (BCS70), own calculations.

Note: $p$-values in parentheses $* p<0.10, * * p<0.05, * * * p<0.01$. The OLS regressions include controls for mother's age, mother's age squared, region of living and parental socioeconomic background at birth, the personality traits agreeableness and extraversion measured at the age of 16 as well as cognitive ability measured at the age of 10 .

An increase in thoughts of helplessness between the ages of 16 and 30 is considered if the indicator for helplessness increases by more than 1 standard deviation, while a decrease is considered if it falls by more than 1 standard deviation. The reference group are those individuals who do not experience an increase or decrease of helplessness by 1 standard deviation between the ages of 16 and 30. First, gender differences are particularly addressed in Table 6.6 by calculating interaction terms between a change in helplessness and a male dummy. Second, variation by person- 
ality traits are targeted in Appendix Table 6.16 by calculating interaction terms between a change in helplessness and the previously introduced groups comprising low versus high emotionally stable and conscientious individuals.

Table 6.6 shows that an increase in helplessness between the ages of 16 and 30 is significantly positive related to an increase in mental health problems at the ages of 30, 34 and 42, while a helplessness decline is associated with a mental ill-health reduction. The size of the coefficients is economically substantial. An increase (decrease) in helplessness is related to an increase (decrease) in mental ill-health by around 0.5 of a standard deviation at the age of 30 , even after controlling for mental ill-health at the age of 16 . At the ages of 34 and 42 , the coefficient size is decreasing, but remains highly significant. This suggests that thoughts of helplessness are volatile throughout life. However, the results clearly show that the tendency to have pessimistic expectations is predictive of mental health problems, which is in line with the psychological literature and the economic model. There are no significant gender differences in this association.

Appendix Table 6.16 reveals similar patterns for all the personality groups. Thus, an increase in thoughts of helplessness between the ages of 16 and 30 is significantly positive related to an increase in mental ill-health, while a decline in thoughts of helplessness is significantly negative associated with mental ill-health in comparison to those who do not experience a change in thoughts of helplessness between the ages of 16 and 30 .

\subsection{Conclusion}

Given the association between emotional stability, conscientiousness and mental health problems, the question about the role of pessimistic expectations arises. It is investigated by analyzing the relation (1) between emotional stability and conscientiousness at the age of 10 and 16 and pessimistic expectations at the age of 16, (2) between pessimistic expectations at the age of 16 and mental ill-health at later ages and (3) between a change in pessimistic expectations between the ages of 16 and 30 and mental health problems at later ages.

The main results are summarized as follows: First, the analysis shows that pessimistic expectations are quite common among teenagers. The probability of experiencing thoughts of helplessness is on average $55 \%$, while the probability of experi- 
encing thoughts of hopelessness is on average $28 \%$. After controlling for personality traits and other factors, boys have a 9 percentage points lower risk of experiencing thoughts of helplessness compared to girls. For thoughts of hopelessness, no gender differences are found.

The relation between personality and pessimistic expectations varies between males and females. At the age of 16, low emotional stability is significantly positively associated with helplessness for girls, but not for boys, while it is significantly related to hopelessness for both genders.

Second, pessimistic expectations significantly predict mental health outcomes after controlling for personality. The association between thoughts of helplessness and mental health problems is (borderline) significantly stronger for women compared to men. Furthermore, the results suggest that pessimistic expectations partially mediate the relation between personality and mental ill-health. These findings are in line with the results in Chapter 4 . The results indicate that gender differences in pessimistic expectations as well as the stronger vulnerability to pessimistic expectations for women compared to men might be able to at least partly explain gender differences in educational achievement and mental health outcomes.

Third, the study finds that an increase in pessimistic expectations between the ages of 16 and 30 is significantly related to an increase in mental ill-health, while a decrease in pessimistic expectations is significantly associated to a decrease in mental health problems at the ages of 30,34 and 42 .

Finally, Chapters 4 and 6 slightly indicate that low emotionally stable, but more conscientious men who have pessimistic expectations have (borderline) significantly better education and mental health outcomes compared to women with the same characteristics. One might argue that these men are simply not affected by low emotional stability and pessimistic expectations. However, the analysis reveals that pessimistic expectations predict poorer education and mental health outcome for both genders. Therefore, it is likely that other mechanisms are at place. Arguing in terms of the economic model, it is likely that less emotionally stable, but more conscientious men who are pessimistic at the age of 16 act in contrast to the model.

They might force themselves to overcome pessimistic expectations more intensively by putting effort instead of avoiding challenging situations. This is likely to result in more experiences of success and mastering, e.g. with regard to improved education 
outcomes, which is found in Chapter 4. Furthermore, it is likely that pessimistic expectations become adjusted in the long run which might boost control in challenging situations and is likely to reduce sustained stress. Thus, mental health problems are expected to become less prevalent over time, which is found in Chapter 5.

This suggests that adjusting pessimistic expectations - both on either personal development or more encouraging environmental conditions - is likely to boost education and mental health outcomes in life. 


\subsection{Appendix}

\subsubsection{Data Description}

Table 6.7: Descriptive Statistics (Female, Age 16)

\begin{tabular}{|c|c|c|c|c|c|c|c|c|c|c|}
\hline \multirow[b]{2}{*}{ Variable Names } & \multicolumn{5}{|c|}{ Working Sample } & \multicolumn{5}{|c|}{ Unrestricted Sample } \\
\hline & $\mathrm{N}$ & Mean & $\mathrm{SD}$ & Min & Max & $\mathrm{N}$ & Mean & $\mathrm{SD}$ & Min & Max \\
\hline Malaise Score (Age 16) & 1245 & -0.04 & 0.99 & -1.67 & 4.04 & 2711 & 0.00 & 1.00 & -1.67 & 4.75 \\
\hline Helplessness (Age 16) & 1245 & 0.56 & 0.50 & 0.00 & 1.00 & 2907 & 0.58 & 0.49 & 0.00 & 1.00 \\
\hline Hopelessness (Age 16) & 1245 & 0.29 & 0.45 & 0.00 & 1.00 & 4036 & 0.33 & 0.47 & 0.00 & 1.00 \\
\hline Emotional Stability (ES, Age 10) & 1245 & 0.09 & 0.93 & -3.51 & 1.19 & 5769 & 0.00 & 1.00 & -3.81 & 1.21 \\
\hline Conscientiousness (C, Age 10) & 1245 & 0.14 & 0.88 & -3.78 & 1.16 & 5769 & -0.00 & 1.00 & -3.78 & 1.16 \\
\hline Agreeableness (A, Age 10) & 1245 & 0.09 & 0.89 & -5.81 & 1.20 & 5769 & 0.00 & 1.00 & -6.32 & 1.36 \\
\hline Extraversion (E, Age 10) & 1245 & 0.02 & 0.99 & -3.15 & 1.45 & 5769 & -0.00 & 1.00 & -3.49 & 1.45 \\
\hline Emotional Stability (ES, Age 16) & 1245 & 0.12 & 0.87 & -3.79 & 0.85 & 3669 & 0.00 & 1.00 & -3.82 & 0.85 \\
\hline Conscientiousness (C, Age 16) & 1245 & 0.13 & 0.88 & -4.95 & 0.67 & 3669 & -0.00 & 1.00 & -4.95 & 0.67 \\
\hline Agreeableness (A, Age 16) & 1245 & 0.15 & 0.86 & -6.33 & 0.53 & 3669 & -0.00 & 1.00 & -6.36 & 0.53 \\
\hline Extraversion (E, Age 16) & 1245 & 0.03 & 0.97 & -4.49 & 1.01 & 3669 & -0.00 & 1.00 & -4.49 & 1.01 \\
\hline High ES, High C (Age 16) & 1245 & 0.59 & 0.49 & 0.00 & 1.00 & 3669 & 0.52 & 0.50 & 0.00 & 1.00 \\
\hline High ES, Low C (Age 16) & 1245 & 0.10 & 0.30 & 0.00 & 1.00 & 3669 & 0.12 & 0.32 & 0.00 & 1.00 \\
\hline Low ES, High C (Age 16) & 1245 & 0.15 & 0.36 & 0.00 & 1.00 & 3669 & 0.16 & 0.37 & 0.00 & 1.00 \\
\hline Low ES, Low C (Age 16) & 1245 & 0.16 & 0.37 & 0.00 & 1.00 & 3669 & 0.20 & 0.40 & 0.00 & 1.00 \\
\hline Cognitive Ability (Age 10) & 1245 & 0.41 & 0.86 & -3.31 & 2.50 & 5637 & 0.00 & 1.00 & -4.60 & 2.55 \\
\hline Higher SES Parents (Birth) & 1245 & 0.25 & 0.43 & 0.00 & 1.00 & 8268 & 0.18 & 0.39 & 0.00 & 1.00 \\
\hline Region (Birth) & 1245 & 5.70 & 2.64 & 1.00 & 10.00 & 8279 & 5.87 & 2.69 & 1.00 & 11.00 \\
\hline Age Mother & 1245 & 23.01 & 3.90 & 14.00 & 39.00 & 8235 & 22.19 & 4.03 & 12.00 & 43.00 \\
\hline
\end{tabular}

Source: BCS70, own calculations. SES refers to the term socioeconomic status.

Table 6.8: Descriptive Statistics (Male, Age 16)

\begin{tabular}{|c|c|c|c|c|c|c|c|c|c|c|}
\hline \multirow[b]{2}{*}{ Variable Names } & \multicolumn{5}{|c|}{ Working Sample } & \multicolumn{5}{|c|}{ Unrestricted Sample } \\
\hline & $\mathrm{N}$ & Mean & SD & Min & Max & $\mathrm{N}$ & Mean & $\mathrm{SD}$ & Min & $\operatorname{Max}$ \\
\hline Malaise Score (Age 16) & 806 & -0.02 & 0.96 & -1.35 & 3.73 & 1962 & 0.00 & 1.00 & -1.35 & 4.46 \\
\hline Helplessness (Age 16) & 806 & 0.52 & 0.50 & 0.00 & 1.00 & 2179 & 0.53 & 0.50 & 0.00 & 1.00 \\
\hline Hopelessness (Age 16) & 806 & 0.27 & 0.44 & 0.00 & 1.00 & 3840 & 0.31 & 0.46 & 0.00 & 1.00 \\
\hline Emotional Stability (ES, Age 10) & 806 & 0.13 & 0.91 & -3.03 & 1.22 & 6027 & -0.00 & 1.00 & -3.25 & 1.25 \\
\hline
\end{tabular}




\begin{tabular}{|c|c|c|c|c|c|c|c|c|c|c|}
\hline \multirow[b]{2}{*}{ Variable Names } & \multicolumn{5}{|c|}{ Working Sample } & \multicolumn{5}{|c|}{ Unrestricted Sample } \\
\hline & $\mathrm{N}$ & Mean & $\mathrm{SD}$ & Min & Max & $\mathrm{N}$ & Mean & $\mathrm{SD}$ & Min & Max \\
\hline Conscientiousness (C, Age 10) & 806 & 0.23 & 0.89 & -2.80 & 1.26 & 6027 & -0.00 & 1.00 & -3.03 & 1.26 \\
\hline Agreeableness (A, Age 10) & 806 & 0.18 & 0.73 & -4.59 & 1.26 & 6027 & -0.00 & 1.00 & -5.63 & 1.34 \\
\hline Extraversion (E, Age 10) & 806 & 0.03 & 0.98 & -3.00 & 1.48 & 6027 & -0.00 & 1.00 & -3.75 & 1.48 \\
\hline Emotional Stability (ES, Age 16) & 806 & 0.09 & 0.95 & -4.18 & 0.83 & 3463 & 0.00 & 1.00 & -4.18 & 0.83 \\
\hline Conscientiousness (C, Age 16) & 806 & 0.17 & 0.86 & -3.89 & 0.77 & 3463 & 0.00 & 1.00 & -4.40 & 0.77 \\
\hline Agreeableness (A, Age 16) & 806 & 0.20 & 0.65 & -3.78 & 0.48 & 3463 & 0.00 & 1.00 & -6.57 & 0.48 \\
\hline Extraversion (E, Age 16) & 806 & -0.01 & 0.96 & -4.61 & 0.92 & 3463 & 0.00 & 1.00 & -5.33 & 0.92 \\
\hline High ES, High C (Age 16) & 806 & 0.53 & 0.50 & 0.00 & 1.00 & 3463 & 0.47 & 0.50 & 0.00 & 1.00 \\
\hline High ES, Low C (Age 16) & 806 & 0.15 & 0.35 & 0.00 & 1.00 & 3463 & 0.16 & 0.37 & 0.00 & 1.00 \\
\hline Low ES, High C (Age 16) & 806 & 0.15 & 0.36 & 0.00 & 1.00 & 3463 & 0.15 & 0.36 & 0.00 & 1.00 \\
\hline Low ES, Low C (Age 16) & 806 & 0.17 & 0.37 & 0.00 & 1.00 & 3463 & 0.22 & 0.41 & 0.00 & 1.00 \\
\hline Cognitive Ability (Age 10) & 806 & 0.43 & 0.88 & -3.09 & 2.23 & 5961 & -0.00 & 1.00 & -3.55 & 2.33 \\
\hline Higher SES Parents (Birth) & 806 & 0.22 & 0.41 & 0.00 & 1.00 & 8896 & 0.18 & 0.38 & 0.00 & 1.00 \\
\hline Region (Birth) & 806 & 5.73 & 2.72 & 1.00 & 10.00 & 8906 & 5.90 & 2.71 & 1.00 & 11.00 \\
\hline Age Mother & 806 & 23.21 & 4.06 & 14.00 & 42.00 & 8832 & 22.11 & 4.03 & 13.00 & 47.00 \\
\hline
\end{tabular}

Source: BCS70, own calculations. SES refers to the term socioeconomic status.

Table 6.9: Descriptive Statistics (Age 16)

\begin{tabular}{lllllllllll}
\hline \multirow{2}{*}{ Variable Names } & \multicolumn{3}{c}{ Working Sample } & \multicolumn{7}{c}{ Unrestricted Sample } \\
& $\mathrm{N}$ & Mean & $\mathrm{SD}$ & $\mathrm{Min}$ & $\mathrm{Max}$ & $\mathrm{N}$ & $\mathrm{Mean}$ & $\mathrm{SD}$ & Min & Max \\
\hline Malaise Score (Age 16) & 2051 & -0.03 & 0.98 & -1.51 & 4.15 & 4673 & 0.00 & 1.00 & -1.51 & 4.86 \\
Helplessness (Age 16) & 2051 & 0.55 & 0.50 & 0.00 & 1.00 & 5086 & 0.56 & 0.50 & 0.00 & 1.00 \\
Hopelessness (Age 16) & 2051 & 0.28 & 0.45 & 0.00 & 1.00 & 7876 & 0.32 & 0.47 & 0.00 & 1.00 \\
Emotional Stability (ES, Age 10) & 2051 & 0.13 & 0.91 & -3.23 & 1.26 & 11796 & 0.00 & 1.00 & -3.51 & 1.34 \\
Conscientiousness (C, Age 10) & 2051 & 0.21 & 0.86 & -3.31 & 1.20 & 11796 & 0.00 & 1.00 & -3.35 & 1.20 \\
Agreeableness (A, Age 10) & 2051 & 0.14 & 0.78 & -5.79 & 1.27 & 11796 & 0.00 & 1.00 & -6.48 & 1.38 \\
Extraversion (E, Age 10) & 2051 & 0.02 & 0.99 & -3.27 & 1.47 & 11796 & -0.00 & 1.00 & -3.62 & 1.47 \\
Emotional Stability (ES, Age 16) & 2051 & 0.10 & 0.91 & -4.00 & 0.85 & 7132 & -0.00 & 1.00 & -4.00 & 0.85 \\
Conscientiousness (C, Age 16) & 2051 & 0.16 & 0.86 & -4.65 & 0.72 & 7132 & -0.00 & 1.00 & -4.65 & 0.72 \\
Agreeableness (A, Age 16) & 2051 & 0.18 & 0.78 & -6.54 & 0.52 & 7132 & 0.00 & 1.00 & -6.61 & 0.53 \\
Extraversion (E, Age 16) & 2051 & 0.00 & 0.97 & -4.82 & 0.96 & 7132 & 0.00 & 1.00 & -4.82 & 0.96 \\
High ES, High C (Age 16) & 2051 & 0.56 & 0.50 & 0.00 & 1.00 & 7132 & 0.49 & 0.50 & 0.00 & 1.00 \\
High ES, Low C (Age 16) & 2051 & 0.12 & 0.32 & 0.00 & 1.00 & 7132 & 0.14 & 0.35 & 0.00 & 1.00 \\
Low ES, High C (Age 16) & 2051 & 0.16 & 0.36 & 0.00 & 1.00 & 7132 & 0.16 & 0.37 & 0.00 & 1.00 \\
& & & & & & & & Continued on next page ...
\end{tabular}




\begin{tabular}{|c|c|c|c|c|c|c|c|c|c|c|}
\hline \multirow[b]{2}{*}{ Variable Names } & \multicolumn{5}{|c|}{ Working Sample } & \multicolumn{5}{|c|}{ Unrestricted Sample } \\
\hline & $\mathrm{N}$ & Mean & $\mathrm{SD}$ & Min & $\operatorname{Max}$ & $\mathrm{N}$ & Mean & SD & Min & $\operatorname{Max}$ \\
\hline Low ES, Low C (Age 16) & 2051 & 0.17 & 0.37 & 0.00 & 1.00 & 7132 & 0.21 & 0.41 & 0.00 & 1.00 \\
\hline Male & 2051 & 0.39 & 0.49 & 0.00 & 1.00 & 17185 & 0.52 & 0.50 & 0.00 & 1.00 \\
\hline Cognitive Ability (Age 10) & 2051 & 0.42 & 0.86 & -3.21 & 2.44 & 11598 & -0.00 & 1.00 & -4.42 & 2.49 \\
\hline Higher SES Parents (Birth) & 2051 & 0.24 & 0.42 & 0.00 & 1.00 & 17164 & 0.18 & 0.39 & 0.00 & 1.00 \\
\hline Region (Birth) & 2051 & 5.71 & 2.67 & 1.00 & 10.00 & 17185 & 5.89 & 2.70 & 1.00 & 11.00 \\
\hline Age Mother & 2051 & 23.09 & 3.97 & 14.00 & 42.00 & 17067 & 22.15 & 4.03 & 12.00 & 47.00 \\
\hline
\end{tabular}

Source: BCS70, own calculations. SES refers to the term socioeconomic status.

Table 6.10: Descriptive Statistics (Age 26)

\begin{tabular}{|c|c|c|c|c|c|c|c|c|c|c|}
\hline \multirow[b]{2}{*}{ Variable Names } & \multicolumn{5}{|c|}{ Working Sample } & \multicolumn{5}{|c|}{ Unrestricted Sample } \\
\hline & $\mathrm{N}$ & Mean & $\mathrm{SD}$ & Min & $\operatorname{Max}$ & $\mathrm{N}$ & Mean & SD & Min & Max \\
\hline Malaise Score (Age 26) & 1634 & -0.04 & 0.95 & -1.00 & 4.07 & 8044 & -0.00 & 1.00 & -1.00 & 4.07 \\
\hline Helplessness (Age 16) & 1634 & 0.54 & 0.50 & 0.00 & 1.00 & 5086 & 0.56 & 0.50 & 0.00 & 1.00 \\
\hline Hopelessness (Age 16) & 1634 & 0.28 & 0.45 & 0.00 & 1.00 & 7876 & 0.32 & 0.47 & 0.00 & 1.00 \\
\hline Emotional Stability (ES, Age 10) & 1634 & 0.16 & 0.89 & -3.23 & 1.26 & 11796 & 0.00 & 1.00 & -3.51 & 1.34 \\
\hline Conscientiousness (C, Age 10) & 1634 & 0.24 & 0.83 & -3.11 & 1.20 & 11796 & 0.00 & 1.00 & -3.35 & 1.20 \\
\hline Agreeableness (A, Age 10) & 1634 & 0.17 & 0.74 & -5.39 & 1.27 & 11796 & 0.00 & 1.00 & -6.48 & 1.38 \\
\hline Extraversion (E, Age 10) & 1634 & 0.02 & 0.99 & -3.27 & 1.47 & 11796 & -0.00 & 1.00 & -3.62 & 1.47 \\
\hline Emotional Stability (ES, Age 16) & 1634 & 0.11 & 0.90 & -4.00 & 0.85 & 7132 & -0.00 & 1.00 & -4.00 & 0.85 \\
\hline Conscientiousness (C, Age 16) & 1634 & 0.20 & 0.84 & -4.65 & 0.72 & 7132 & -0.00 & 1.00 & -4.65 & 0.72 \\
\hline Agreeableness (A, Age 16) & 1634 & 0.22 & 0.75 & -6.54 & 0.52 & 7132 & 0.00 & 1.00 & -6.61 & 0.53 \\
\hline Extraversion (E, Age 16) & 1634 & 0.00 & 0.97 & -4.82 & 0.96 & 7132 & 0.00 & 1.00 & -4.82 & 0.96 \\
\hline High ES, High C (Age 16) & 1634 & 0.57 & 0.49 & 0.00 & 1.00 & 7132 & 0.49 & 0.50 & 0.00 & 1.00 \\
\hline High ES, Low C (Age 16) & 1634 & 0.11 & 0.31 & 0.00 & 1.00 & 7132 & 0.14 & 0.35 & 0.00 & 1.00 \\
\hline Low ES, High C (Age 16) & 1634 & 0.16 & 0.37 & 0.00 & 1.00 & 7132 & 0.16 & 0.37 & 0.00 & 1.00 \\
\hline Low ES, Low C (Age 16) & 1634 & 0.16 & 0.37 & 0.00 & 1.00 & 7132 & 0.21 & 0.41 & 0.00 & 1.00 \\
\hline Male & 1634 & 0.37 & 0.48 & 0.00 & 1.00 & 17185 & 0.52 & 0.50 & 0.00 & 1.00 \\
\hline Cognitive Ability (Age 10) & 1634 & 0.47 & 0.84 & -3.21 & 2.44 & 11598 & -0.00 & 1.00 & -4.42 & 2.49 \\
\hline Higher SES Parents (Birth) & 1634 & 0.24 & 0.43 & 0.00 & 1.00 & 17164 & 0.18 & 0.39 & 0.00 & 1.00 \\
\hline Region (Birth) & 1634 & 5.66 & 2.68 & 1.00 & 10.00 & 17185 & 5.89 & 2.70 & 1.00 & 11.00 \\
\hline Age Mother & 1634 & 23.26 & 3.97 & 15.00 & 42.00 & 17067 & 22.15 & 4.03 & 12.00 & 47.00 \\
\hline
\end{tabular}

Source: BCS70, own calculations. SES refers to the term socioeconomic status. 
Table 6.11: Descriptive Statistics (Age 30)

\begin{tabular}{|c|c|c|c|c|c|c|c|c|c|c|}
\hline \multirow[b]{2}{*}{ Variable Names } & \multicolumn{5}{|c|}{ Working Sample } & \multicolumn{5}{|c|}{ Unrestricted Sample } \\
\hline & $\mathrm{N}$ & Mean & SD & Min & Max & $\mathrm{N}$ & Mean & SD & Min & $\operatorname{Max}$ \\
\hline Malaise Score (Age 30) & 2050 & -0.08 & 0.93 & -0.88 & 4.24 & 10251 & -0.00 & 1.00 & -0.88 & 4.24 \\
\hline Helplessness (Age 16) & 2050 & 0.55 & 0.50 & 0.00 & 1.00 & 5086 & 0.56 & 0.50 & 0.00 & 1.00 \\
\hline Hopelessness (Age 16) & 2050 & 0.28 & 0.45 & 0.00 & 1.00 & 7876 & 0.32 & 0.47 & 0.00 & 1.00 \\
\hline Emotional Stability (ES, Age 10) & 2050 & 0.13 & 0.91 & -3.23 & 1.26 & 11796 & 0.00 & 1.00 & -3.51 & 1.34 \\
\hline Conscientiousness (C, Age 10) & 2050 & 0.21 & 0.86 & -3.31 & 1.20 & 11796 & 0.00 & 1.00 & -3.35 & 1.20 \\
\hline Agreeableness (A, Age 10) & 2050 & 0.14 & 0.78 & -5.79 & 1.27 & 11796 & 0.00 & 1.00 & -6.48 & 1.38 \\
\hline Extraversion (E, Age 10) & 2050 & 0.02 & 0.99 & -3.27 & 1.47 & 11796 & -0.00 & 1.00 & -3.62 & 1.47 \\
\hline Emotional Stability (ES, Age 16) & 2050 & 0.10 & 0.91 & -4.00 & 0.85 & 7132 & -0.00 & 1.00 & -4.00 & 0.85 \\
\hline Conscientiousness (C, Age 16) & 2050 & 0.16 & 0.86 & -4.65 & 0.72 & 7132 & -0.00 & 1.00 & -4.65 & 0.72 \\
\hline Agreeableness (A, Age 16) & 2050 & 0.18 & 0.78 & -6.54 & 0.52 & 7132 & 0.00 & 1.00 & -6.61 & 0.53 \\
\hline Extraversion (E, Age 16) & 2050 & 0.00 & 0.97 & -4.82 & 0.96 & 7132 & 0.00 & 1.00 & -4.82 & 0.96 \\
\hline High ES, High C (Age 16) & 2050 & 0.56 & 0.50 & 0.00 & 1.00 & 7132 & 0.49 & 0.50 & 0.00 & 1.00 \\
\hline High ES, Low C (Age 16) & 2050 & 0.12 & 0.32 & 0.00 & 1.00 & 7132 & 0.14 & 0.35 & 0.00 & 1.00 \\
\hline Low ES, High C (Age 16) & 2050 & 0.16 & 0.36 & 0.00 & 1.00 & 7132 & 0.16 & 0.37 & 0.00 & 1.00 \\
\hline Low ES, Low C (Age 16) & 2050 & 0.17 & 0.37 & 0.00 & 1.00 & 7132 & 0.21 & 0.41 & 0.00 & 1.00 \\
\hline Male & 2050 & 0.39 & 0.49 & 0.00 & 1.00 & 17185 & 0.52 & 0.50 & 0.00 & 1.00 \\
\hline Cognitive Ability (Age 10) & 2050 & 0.42 & 0.86 & -3.21 & 2.44 & 11598 & -0.00 & 1.00 & -4.42 & 2.49 \\
\hline Higher SES Parents (Birth) & 2050 & 0.24 & 0.42 & 0.00 & 1.00 & 17164 & 0.18 & 0.39 & 0.00 & 1.00 \\
\hline Region (Birth) & 2050 & 5.71 & 2.67 & 1.00 & 10.00 & 17185 & 5.89 & 2.70 & 1.00 & 11.00 \\
\hline Age Mother & 2050 & 23.09 & 3.97 & 14.00 & 42.00 & 17067 & 22.15 & 4.03 & 12.00 & 47.00 \\
\hline
\end{tabular}

Source: BCS70, own calculations. SES refers to the term socioeconomic status.

Table 6.12: Descriptive Statistics (Age 34)

\begin{tabular}{|c|c|c|c|c|c|c|c|c|c|c|}
\hline \multirow[b]{2}{*}{ Variable Names } & \multicolumn{5}{|c|}{ Working Sample } & \multicolumn{5}{|c|}{ Unrestricted Sample } \\
\hline & $\mathrm{N}$ & Mean & $\mathrm{SD}$ & Min & Max & $\mathrm{N}$ & Mean & $\mathrm{SD}$ & Min & Max \\
\hline Malaise Score (Age 34) & 1783 & -0.09 & 0.94 & -0.88 & 3.87 & 8866 & 0.00 & 1.00 & -0.88 & 3.87 \\
\hline Helplessness (Age 16) & 1783 & 0.55 & 0.50 & 0.00 & 1.00 & 5086 & 0.56 & 0.50 & 0.00 & 1.00 \\
\hline Hopelessness (Age 16) & 1783 & 0.28 & 0.45 & 0.00 & 1.00 & 7876 & 0.32 & 0.47 & 0.00 & 1.00 \\
\hline Emotional Stability (ES, Age 10) & 1783 & 0.14 & 0.90 & -3.23 & 1.26 & 11796 & 0.00 & 1.00 & -3.51 & 1.34 \\
\hline Conscientiousness (C, Age 10) & 1783 & 0.21 & 0.87 & -3.31 & 1.20 & 11796 & 0.00 & 1.00 & -3.35 & 1.20 \\
\hline Agreeableness (A, Age 10) & 1783 & 0.16 & 0.75 & -5.79 & 1.26 & 11796 & 0.00 & 1.00 & -6.48 & 1.38 \\
\hline Extraversion (E, Age 10) & 1783 & 0.01 & 1.00 & -3.27 & 1.47 & 11796 & -0.00 & 1.00 & -3.62 & 1.47 \\
\hline
\end{tabular}




\begin{tabular}{|c|c|c|c|c|c|c|c|c|c|c|}
\hline \multirow[b]{2}{*}{ Variable Names } & \multicolumn{5}{|c|}{ Working Sample } & \multicolumn{5}{|c|}{ Unrestricted Sample } \\
\hline & $\mathrm{N}$ & Mean & SD & Min & Max & $\mathrm{N}$ & Mean & $\mathrm{SD}$ & Min & Max \\
\hline Emotional Stability (ES, Age 16) & 1783 & 0.11 & 0.90 & -4.00 & 0.85 & 7132 & -0.00 & 1.00 & -4.00 & 0.85 \\
\hline Conscientiousness (C, Age 16) & 1783 & 0.17 & 0.86 & -4.65 & 0.72 & 7132 & -0.00 & 1.00 & -4.65 & 0.72 \\
\hline Agreeableness (A, Age 16) & 1783 & 0.20 & 0.76 & -6.54 & 0.52 & 7132 & 0.00 & 1.00 & -6.61 & 0.53 \\
\hline Extraversion (E, Age 16) & 1783 & 0.01 & 0.97 & -4.32 & 0.96 & 7132 & 0.00 & 1.00 & -4.82 & 0.96 \\
\hline High ES, High C (Age 16) & 1783 & 0.56 & 0.50 & 0.00 & 1.00 & 7132 & 0.49 & 0.50 & 0.00 & 1.00 \\
\hline High ES, Low C (Age 16) & 1783 & 0.12 & 0.33 & 0.00 & 1.00 & 7132 & 0.14 & 0.35 & 0.00 & 1.00 \\
\hline Low ES, High C (Age 16) & 1783 & 0.16 & 0.37 & 0.00 & 1.00 & 7132 & 0.16 & 0.37 & 0.00 & 1.00 \\
\hline Low ES, Low C (Age 16) & 1783 & 0.16 & 0.36 & 0.00 & 1.00 & 7132 & 0.21 & 0.41 & 0.00 & 1.00 \\
\hline Male & 1783 & 0.38 & 0.49 & 0.00 & 1.00 & 17185 & 0.52 & 0.50 & 0.00 & 1.00 \\
\hline Cognitive Ability (Age 10) & 1783 & 0.43 & 0.85 & -3.16 & 2.44 & 11598 & -0.00 & 1.00 & -4.42 & 2.49 \\
\hline Higher SES Parents (Birth) & 1783 & 0.24 & 0.43 & 0.00 & 1.00 & 17164 & 0.18 & 0.39 & 0.00 & 1.00 \\
\hline Region (Birth) & 1783 & 5.70 & 2.65 & 1.00 & 10.00 & 17185 & 5.89 & 2.70 & 1.00 & 11.00 \\
\hline Age Mother & 1783 & 23.21 & 3.93 & 14.00 & 42.00 & 17067 & 22.15 & 4.03 & 12.00 & 47.00 \\
\hline
\end{tabular}

Source: BCS70, own calculations. SES refers to the term socioeconomic status.

Table 6.13: Descriptive Statistics (Age 42)

\begin{tabular}{lcccccccccc}
\hline & \multicolumn{3}{l}{ Working Sample } & \multicolumn{7}{c}{ Unrestricted Sample } \\
Variable Names & N & Mean & SD & Min & Max & N & Mean & SD & Min & Max \\
\hline Malaise Score (Age 42) & 1592 & -0.07 & 0.93 & -0.93 & 3.57 & 7935 & 0.00 & 1.00 & -0.93 & 3.57 \\
Helplessness (Age 16) & 1592 & 0.55 & 0.50 & 0.00 & 1.00 & 5086 & 0.56 & 0.50 & 0.00 & 1.00 \\
Hopelessness (Age 16) & 1592 & 0.28 & 0.45 & 0.00 & 1.00 & 7876 & 0.32 & 0.47 & 0.00 & 1.00 \\
Emotional Stability (ES, Age 10) & 1592 & 0.16 & 0.88 & -3.23 & 1.26 & 11796 & 0.00 & 1.00 & -3.51 & 1.34 \\
Conscientiousness (C, Age 10) & 1592 & 0.22 & 0.86 & -3.31 & 1.20 & 11796 & 0.00 & 1.00 & -3.35 & 1.20 \\
Agreeableness (A, Age 10) & 1592 & 0.18 & 0.72 & -5.42 & 1.26 & 11796 & 0.00 & 1.00 & -6.48 & 1.38 \\
Extraversion (E, Age 10) & 1592 & 0.02 & 1.00 & -3.27 & 1.47 & 11796 & -0.00 & 1.00 & -3.62 & 1.47 \\
Emotional Stability (ES, Age 16) & 1592 & 0.11 & 0.91 & -4.00 & 0.85 & 7132 & -0.00 & 1.00 & -4.00 & 0.85 \\
Conscientiousness (C, Age 16) & 1592 & 0.19 & 0.84 & -4.65 & 0.72 & 7132 & -0.00 & 1.00 & -4.65 & 0.72 \\
Agreeableness (A, Age 16) & 1592 & 0.21 & 0.72 & -6.54 & 0.52 & 7132 & 0.00 & 1.00 & -6.61 & 0.53 \\
Extraversion (E, Age 16) & 1592 & -0.01 & 0.97 & -4.32 & 0.96 & 7132 & 0.00 & 1.00 & -4.82 & 0.96 \\
High ES, High C (Age 16) & 1592 & 0.57 & 0.50 & 0.00 & 1.00 & 7132 & 0.49 & 0.50 & 0.00 & 1.00 \\
High ES, Low C (Age 16) & 1592 & 0.11 & 0.32 & 0.00 & 1.00 & 7132 & 0.14 & 0.35 & 0.00 & 1.00 \\
Low ES, High C (Age 16) & 1592 & 0.16 & 0.37 & 0.00 & 1.00 & 7132 & 0.16 & 0.37 & 0.00 & 1.00 \\
Low ES, Low C (Age 16) & 1592 & 0.16 & 0.36 & 0.00 & 1.00 & 7132 & 0.21 & 0.41 & 0.00 & 1.00 \\
Male & 1592 & 0.37 & 0.48 & 0.00 & 1.00 & 17185 & 0.52 & 0.50 & 0.00 & 1.00
\end{tabular}


... Table 6.13 continued

\begin{tabular}{lccccccccccc}
\hline & \multicolumn{3}{c}{ Working Sample } & \multicolumn{4}{c}{ Unrestricted Sample } \\
Variable Names & $\mathrm{N}$ & Mean & SD & Min & Max & N & Mean & SD & Min & Max \\
\hline Cognitive Ability (Age 10) & 1592 & 0.47 & 0.84 & -3.21 & 2.44 & 11598 & -0.00 & 1.00 & -4.42 & 2.49 \\
Higher SES Parents (Birth) & 1592 & 0.25 & 0.43 & 0.00 & 1.00 & 17164 & 0.18 & 0.39 & 0.00 & 1.00 \\
Region (Birth) & 1592 & 5.67 & 2.63 & 1.00 & 10.00 & 17185 & 5.89 & 2.70 & 1.00 & 11.00 \\
Age Mother & 1592 & 23.31 & 3.98 & 14.00 & 42.00 & 17067 & 22.15 & 4.03 & 12.00 & 47.00 \\
\hline
\end{tabular}

Source: BCS70, own calculations. SES refers to the term socioeconomic status.

Table 6.14: Personality Traits at the Ages of 10 and 16

\begin{tabular}{|c|c|c|c|c|}
\hline $\begin{array}{l}\text { Personality } \\
\text { Trait }\end{array}$ & $\begin{array}{l}\text { Correlation } \\
80-86\end{array}$ & $\begin{array}{l}\text { Explained } \\
\text { Variance }\end{array}$ & $\begin{array}{l}\text { Cronbach's } \\
\text { Alpha }\end{array}$ & Items \\
\hline \multicolumn{5}{|c|}{ Emotional Stability } \\
\hline Age 10 & & $53.76 \%$ & 0.83 & R8. Irritable. \\
\hline Age 16 & 0.40 & $55.36 \%$ & 0.84 & $\begin{array}{l}\text { R9. Miserable and tearful. } \\
\text { C8. Requests must be met. } \\
\text { C12. Sullen or sulky. } \\
\text { C16. Changes mood quickly. } \\
\text { C17. Temper unpredictable. }\end{array}$ \\
\hline
\end{tabular}

Conscientiousness

Age 10

$\begin{array}{lll}65.99 \% & 0.83 \\ 0.44 & 63.64 \% & 0.81\end{array}$

0.83

R15. Cannot settle.

Age 16

0.44

$63.64 \%$

C3. Easily distracted.

C13. Fails to finish things.

C19. Difficulty concentrating.

Agreeableness

Age 10

Age 16

0.32

$46.82 \%$

0.81

R3. Destroys belongings.

R4. Frequently fights.

R10. Takes others' belongings.

R14. Often disobedient.

R18. Often tells lies.

R19. Bullies other children.

C11. Interferes with others.

Extraversion

$\begin{array}{llll}\text { Age } 10 & & 37.88 \% & 0.57 \\ \text { Age } 16 & 0.37 & 36.43 \% & 0.55\end{array}$

R5. Not much liked by others.

R6. Often worried.

R7. Rather solitary.

R16. Afraid of new things.

R17. Fussy or over-particular.

Source: BCS70, own calculations based on working sample $\mathrm{n}=2051$.

Note: Items are stated in original terms, but reversely used in the analysis. 
Table 6.15: Correlation between Personality Traits, Help- and Hopelessness, and Mental Health Outcomes

\begin{tabular}{|c|c|c|c|c|c|c|c|}
\hline & HE16 & HO16 & MH16 & MH26 & MH30 & MH34 & MH42 \\
\hline \multicolumn{8}{|l|}{ Emotional Stability } \\
\hline Age 10 & -.08 & -.16 & -.12 & -.17 & -.11 & -.11 & -.13 \\
\hline Age 16 & -.13 & -.18 & -.24 & -.24 & -.20 & -.21 & -.23 \\
\hline R8. Irritable. & -.09 & -.14 & -.18 & -.20 & -.13 & -.15 & -.16 \\
\hline R9. Miserable and tearful. & -.14 & -.12 & -.20 & -.20 & -.20 & -.20 & -.25 \\
\hline C8. Requests must be met. & -.07 & -.14 & -.11 & -.12 & -.11 & -.09 & -.11 \\
\hline C12. Sullen or sulky. & -.11 & -.10 & -.20 & -.16 & -.14 & -.17 & -.21 \\
\hline C16. Changes mood quickly. & -.11 & -.14 & -.21 & -.22 & -.16 & -.17 & -.18 \\
\hline C17. Temper unpredictable. & -.09 & -.14 & -.18 & -.19 & -.14 & -.16 & -.15 \\
\hline \multicolumn{8}{|l|}{ Conscientiousness } \\
\hline Age 10 & - & -.08 & -.05 & -.09 & -.07 & -.11 & -.10 \\
\hline Age 16 & -.08 & -.12 & -.14 & -.16 & -.13 & -.16 & -.11 \\
\hline R15. Cannot settle. & -.07 & -.10 & -.10 & -.12 & -.08 & -.13 & -.09 \\
\hline C3. Easily distracted. & -.07 & -.11 & -.11 & -.14 & -.12 & -.12 & -.09 \\
\hline C13. Fails to finish things. & -.06 & -.08 & -.12 & -.13 & -.12 & -.15 & -.11 \\
\hline C19. Difficulty concentrating. & -.05 & -.09 & -.12 & -.12 & -.09 & -.12 & -.06 \\
\hline \multicolumn{8}{|l|}{ Agreeableness } \\
\hline Age 10 & -.04 & -.10 & -.06 & -.12 & -.08 & -.08 & -.10 \\
\hline Age 16 & -.05 & -.12 & -.10 & -.09 & -.09 & -.11 & -.09 \\
\hline R3. Destroys belongings. & - & -.08 & -.03 & - & - & -.06 & - \\
\hline R4. Frequently fights. & - & -.07 & -.06 & -.05 & -.06 & -.07 & -.09 \\
\hline R10. Takes others' belongings. & - & -.06 & - & - & - & -.06 & -.07 \\
\hline R14. Often disobedient. & - & -.10 & -.10 & -.10 & -.06 & -.08 & -.07 \\
\hline R18. Often tells lies. & - & -.08 & -.08 & -.08 & -.10 & -.07 & -.08 \\
\hline R19. Bullies other children. & - & -.06 & -.05 & - & - & - & - \\
\hline C11. Interferes with others. & -.06 & -.07 & -.08 & -.10 & -.08 & -.12 & - \\
\hline \multicolumn{8}{|l|}{ Extraversion } \\
\hline Age 10 & -.06 & -.10 & -.13 & -.12 & -.12 & -.09 & -.10 \\
\hline Age 16 & -.17 & -.16 & -.23 & -.19 & -.17 & -.15 & -.20 \\
\hline R5. Not much liked. & -.09 & -.04 & -.09 & -.06 & -.11 & -.11 & -.08 \\
\hline R6. Often worried. & -.17 & -.13 & -.24 & -.19 & -.15 & -.14 & -.17 \\
\hline R7. Rather solitary. & -.10 & -.11 & -.11 & -.09 & -.09 & -.05 & -.09 \\
\hline R16. Afraid of new things. & -.08 & -.10 & -.14 & -.14 & -.10 & -.09 & -.12 \\
\hline R17. Fussy or over-particular. & -.05 & -.09 & -.08 & -.08 & -.05 & -.07 & -.10 \\
\hline Helplessness (HE16) & - & - & .40 & .18 & .18 & .18 & .16 \\
\hline Hopelessness (HO16) & - & - & .07 & .13 & .09 & .12 & .15 \\
\hline
\end{tabular}

Source: BCS70, own calculations.

Note: Items are stated in original terms, but reversely used in the analysis. Correlation coefficients reported are significant at the $5 \%$ level or higher. $\mathrm{HE}=$ Helplessness, $\mathrm{HO}=$ Hopelessness, $\mathrm{MH}=$ Mental Health Problems at the ages of 16, 26, 30, 34 and 42 . 


\subsubsection{Robustness Checks}

\subsubsection{Helplessness Change, Personality and Mental Ill- Health}

Table 6.16: Helplessness Change, Personality and Mental Ill-Health

\begin{tabular}{|c|c|c|c|c|c|c|}
\hline Mental Ill-Health Score ... & Age 30 & & Age 34 & & All 42 & \\
\hline Helplessness (Age 16) & $\begin{array}{l}0.445^{* * *} \\
(0.000)\end{array}$ & $\begin{array}{l}0.326^{* * *} \\
(0.000)\end{array}$ & $\begin{array}{l}0.365^{* * *} \\
(0.000)\end{array}$ & $\begin{array}{l}0.247^{* * *} \\
(0.000)\end{array}$ & $\begin{array}{l}0.287^{* * *} \\
(0.000)\end{array}$ & $\begin{array}{l}0.179^{* * *} \\
(0.000)\end{array}$ \\
\hline $\begin{array}{l}\text { High Emotional Stability, } \\
\text { Low Conscientiousness } \\
\text { (Age 16) }\end{array}$ & $\begin{array}{l}0.048 \\
(0.578)\end{array}$ & $\begin{array}{l}0.047 \\
(0.585)\end{array}$ & $\begin{array}{l}0.057 \\
(0.569)\end{array}$ & $\begin{array}{l}0.056 \\
(0.560)\end{array}$ & $\begin{array}{l}0.007 \\
(0.948)\end{array}$ & $\begin{array}{l}-0.009 \\
(0.929)\end{array}$ \\
\hline $\begin{array}{l}\text { Low Emotional Stability, } \\
\text { High Conscientiousness } \\
\text { (Age 16) }\end{array}$ & $\begin{array}{l}0.104 \\
(0.197)\end{array}$ & $\begin{array}{l}0.068 \\
(0.390)\end{array}$ & $\begin{array}{l}0.156 \\
(0.110)\end{array}$ & $\begin{array}{l}0.119 \\
(0.213)\end{array}$ & $\begin{array}{l}0.266^{* * *} \\
(0.009)\end{array}$ & $\begin{array}{l}0.231^{* *} \\
(0.022)\end{array}$ \\
\hline $\begin{array}{l}\text { Low Emotional Stability, } \\
\text { Low Conscientiousness } \\
\text { (Age 16) }\end{array}$ & $\begin{array}{l}0.202^{* *} \\
(0.028)\end{array}$ & $\begin{array}{l}0.158^{*} \\
(0.074)\end{array}$ & $\begin{array}{l}0.318^{* * *} \\
(0.005)\end{array}$ & $\begin{array}{l}0.269^{* *} \\
(0.017)\end{array}$ & $\begin{array}{l}0.379^{* * *} \\
(0.001)\end{array}$ & $\begin{array}{l}0.348^{* * *} \\
(0.002)\end{array}$ \\
\hline $\begin{array}{l}\text { High Emotional Stability, } \\
\text { High Conscientiousness } \\
\text { Helplessness Change } \\
(\text { Age } 30-16)>1\end{array}$ & $\begin{array}{l}0.607^{* * *} \\
(0.000)\end{array}$ & $\begin{array}{l}0.560 * * * \\
(0.000)\end{array}$ & $\begin{array}{l}0.391^{* * *} \\
(0.000)\end{array}$ & $\begin{array}{l}0.348^{* * *} \\
(0.000)\end{array}$ & $\begin{array}{l}0.332^{* * *} \\
(0.000)\end{array}$ & $\begin{array}{l}0.296^{* * *} \\
(0.000)\end{array}$ \\
\hline $\begin{array}{l}\text { High Emotional Stability, } \\
\text { High Conscientiousness } \\
\text { Helplessness Change } \\
\text { (Age } 30-16)<-1\end{array}$ & $\begin{array}{l}-0.614^{* * *} \\
(0.000)\end{array}$ & $\begin{array}{l}-0.548^{* * *} \\
(0.000)\end{array}$ & $\begin{array}{l}-0.458^{* * *} \\
(0.000)\end{array}$ & $\begin{array}{l}-0.391^{* * *} \\
(0.000)\end{array}$ & $\begin{array}{l}-0.310^{* * *} \\
(0.000)\end{array}$ & $\begin{array}{l}-0.239^{* * *} \\
(0.007)\end{array}$ \\
\hline $\begin{array}{l}\text { High Emotional Stability, } \\
\text { Low Conscientiousness } \\
\text { Helplessness Change } \\
(\text { Age } 30-16)>1\end{array}$ & $\begin{array}{l}0.495^{* * *} \\
(0.002)\end{array}$ & $\begin{array}{l}0.457^{* * *} \\
(0.003)\end{array}$ & $\begin{array}{l}0.252 \\
(0.147)\end{array}$ & $\begin{array}{l}0.231 \\
(0.163)\end{array}$ & $\begin{array}{l}0.163 \\
(0.348)\end{array}$ & $\begin{array}{l}0.170 \\
(0.316)\end{array}$ \\
\hline $\begin{array}{l}\text { High Emotional Stability, } \\
\text { Low Conscientiousness } \\
\text { Helplessness Change } \\
\text { (Age } 30-16)<-1\end{array}$ & $\begin{array}{l}-0.518^{* * *} \\
(0.000)\end{array}$ & $\begin{array}{l}-0.482^{* * *} \\
(0.000)\end{array}$ & $\begin{array}{l}-0.445^{* * *} \\
(0.003)\end{array}$ & $\begin{array}{l}-0.404^{* * *} \\
(0.005)\end{array}$ & $\begin{array}{c}-0.298^{*} \\
(0.056)\end{array}$ & $\begin{array}{l}-0.239 \\
(0.115)\end{array}$ \\
\hline Low Emotional Stability, & $0.610^{* * *}$ & $0.555^{* * *}$ & 0.120 & ${ }^{0.062}$ Con & $\begin{array}{c}0.171 \\
\text { tinued on } r\end{array}$ & $\begin{array}{l}0.141 \\
\text { zext page }\end{array}$ \\
\hline
\end{tabular}


... Table 6.16 continued

\begin{tabular}{|c|c|c|c|c|c|c|}
\hline Mental Ill-Health Score ... & Age 30 & & Age 34 & & All 42 & \\
\hline $\begin{array}{l}\text { High Conscientiousness } \\
\text { Helplessness Change } \\
(\text { Age } 30-16)>1\end{array}$ & $(0.000)$ & $(0.000)$ & $(0.422)$ & $(0.673)$ & $(0.251)$ & $(0.341)$ \\
\hline $\begin{array}{l}\text { Low Emotional Stability, } \\
\text { High Conscientiousness } \\
\text { Helplessness Change } \\
\text { (Age } 30-16)<-1\end{array}$ & $\begin{array}{l}-0.524^{* * *} \\
(0.000)\end{array}$ & $\begin{array}{l}-0.513^{* * *} \\
(0.000)\end{array}$ & $\begin{array}{l}-0.492^{* * *} \\
(0.000)\end{array}$ & $\begin{array}{l}-0.471^{* * *} \\
(0.000)\end{array}$ & $\begin{array}{l}-0.410^{* * *} \\
(0.003)\end{array}$ & $\begin{array}{l}-0.384^{* * *} \\
(0.006)\end{array}$ \\
\hline $\begin{array}{l}\text { Low Emotional Stability, } \\
\text { Low Conscientiousness } \\
\text { Helplessness Change } \\
(\text { Age } 30-16)>1\end{array}$ & $\begin{array}{l}0.773^{* * *} \\
(0.000)\end{array}$ & $\begin{array}{l}0.665^{\text {*** }} \\
(0.000)\end{array}$ & $\begin{array}{l}0.435^{* * *} \\
(0.006)\end{array}$ & $\begin{array}{l}0.339^{* *} \\
(0.033)\end{array}$ & $\begin{array}{l}0.247 \\
(0.128)\end{array}$ & $\begin{array}{l}0.145 \\
(0.363)\end{array}$ \\
\hline $\begin{array}{l}\text { Low Emotional Stability, } \\
\text { Low Conscientiousness } \\
\text { Helplessness Change } \\
\text { (Age } 30-16)<-1\end{array}$ & $\begin{array}{l}-0.594^{* * *} \\
(0.000)\end{array}$ & $\begin{array}{l}-0.545^{* * *} \\
(0.000)\end{array}$ & $\begin{array}{l}-0.565^{* * *} \\
(0.000)\end{array}$ & $\begin{array}{l}-0.505^{* * *} \\
(0.001)\end{array}$ & $\begin{array}{l}-0.482^{* * *} \\
(0.004)\end{array}$ & $\begin{array}{l}-0.425^{* * *} \\
(0.009)\end{array}$ \\
\hline Malaise Score (Age 16) & & $\begin{array}{l}0.090^{* * *} \\
(0.000)\end{array}$ & & $\begin{array}{l}0.090 * * * \\
(0.000)\end{array}$ & & $\begin{array}{l}0.077^{* * *} \\
(0.000)\end{array}$ \\
\hline $\begin{array}{l}N \\
R^{2}\end{array}$ & $\begin{array}{l}2050 \\
0198\end{array}$ & $\begin{array}{l}2050 \\
0247\end{array}$ & 1783 & $\begin{array}{l}1783 \\
0188\end{array}$ & 1592 & 1592 \\
\hline$R^{2}$ & 0.198 & 0.247 & 0.141 & 0.188 & 0.114 & 0.147 \\
\hline
\end{tabular}

Source: British Cohort Study (BCS70), own calculations.

Note: $p$-values in parentheses ${ }^{*} p<0.10,{ }^{* *} p<0.05,{ }^{* * *} p<0.01$. The OLS regressions include controls for mother's age, mother's age squared, region of living and parental socioeconomic background at birth, the personality traits agreeableness and extraversion measured at the age of 16 as well as cognitive ability measured at the age of 10 . 


\subsubsection{Fully Restricted Sample}

Table 6.17: Personality Trait Change and Help- and Hopelessness

\begin{tabular}{|c|c|c|c|c|}
\hline \multirow{2}{*}{$\frac{\text { Pessimistic Expectations (Age 16) ... }}{\text { Emotional Stability (Age 10) }}$} & \multicolumn{2}{|c|}{ Helplessness } & \multicolumn{2}{|c|}{ Hopelessness } \\
\hline & $-0.093^{* *}$ & $(0.000)$ & $-0.074^{* * *}$ & $(0.004)$ \\
\hline Conscientiousness (Age 10) & 0.007 & $(0.782)$ & -0.030 & $(0.252)$ \\
\hline Emotional Stability Male (Age 10) & 0.045 & $(0.315)$ & 0.016 & $(0.711)$ \\
\hline Conscientiousness Male (Age 10) & -0.056 & $(0.143)$ & $0.068^{*}$ & $(0.060)$ \\
\hline Emotional Stability (Age 16-10) $>0.5$ & $-0.097^{*}$ & $(0.074)$ & -0.011 & $(0.831)$ \\
\hline Emotional Stability (Age 16-10) $<-0.5$ & -0.012 & $(0.779)$ & $0.139^{* * *}$ & $(0.001)$ \\
\hline Conscientiousness (Age 16-10) $>0.5$ & 0.010 & $(0.858)$ & -0.068 & $(0.188)$ \\
\hline Conscientiousness (Age 16-10) $<-0.5$ & 0.015 & $(0.748)$ & -0.039 & $(0.344)$ \\
\hline Emotional Stability Male (Age 16-10) $>0.5$ & 0.123 & $(0.168)$ & 0.008 & $(0.915)$ \\
\hline Emotional Stability Male (Age 16-10) $<-0.5$ & $0.186^{* *}$ & $(0.023)$ & -0.052 & $(0.499)$ \\
\hline Conscientiousness Male (Age 16-10) $>0.5$ & -0.123 & $(0.182)$ & 0.126 & $(0.129)$ \\
\hline Conscientiousness Male (Age 16-10) $<-0.5$ & 0.066 & $(0.411)$ & 0.044 & $(0.557)$ \\
\hline Male & -0.060 & $(0.369)$ & -0.012 & $(0.849)$ \\
\hline$N$ & 1218 & & 1218 & \\
\hline$R^{2}$ & 0.065 & & 0.072 & \\
\hline
\end{tabular}

Source: British Cohort Study (BCS70), own calculations.

Note: $p$-values in parentheses ${ }^{*} p<0.10,{ }^{* *} p<0.05,{ }^{* * *} p<0.01$. The OLS regressions include controls for mother's age, mother's age squared, region of living and parental socioeconomic background at birth, the personality traits agreeableness and extraversion measured at the ages of 10 and 16 as well as cognitive ability measured at the age of 10 . 


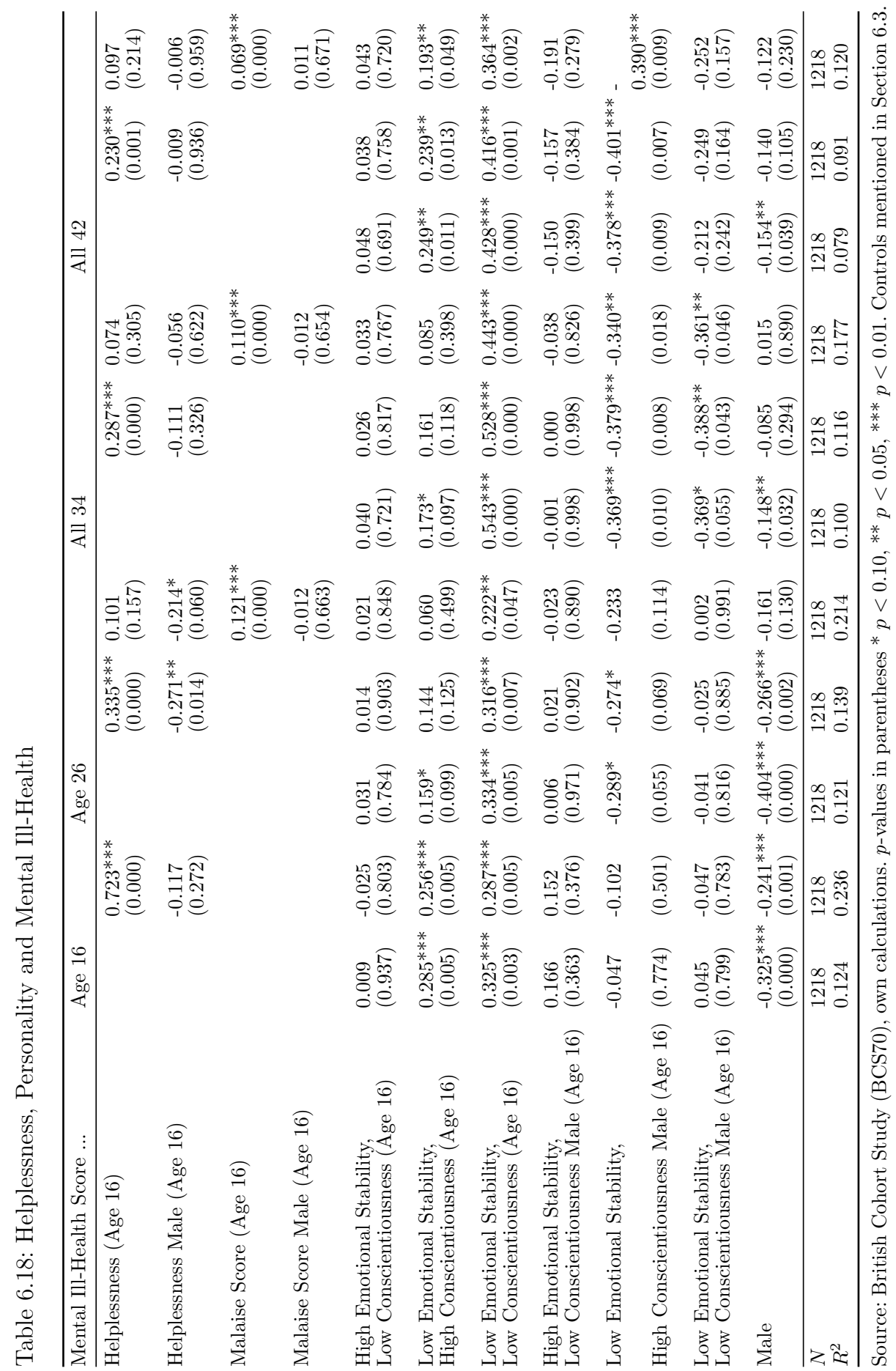




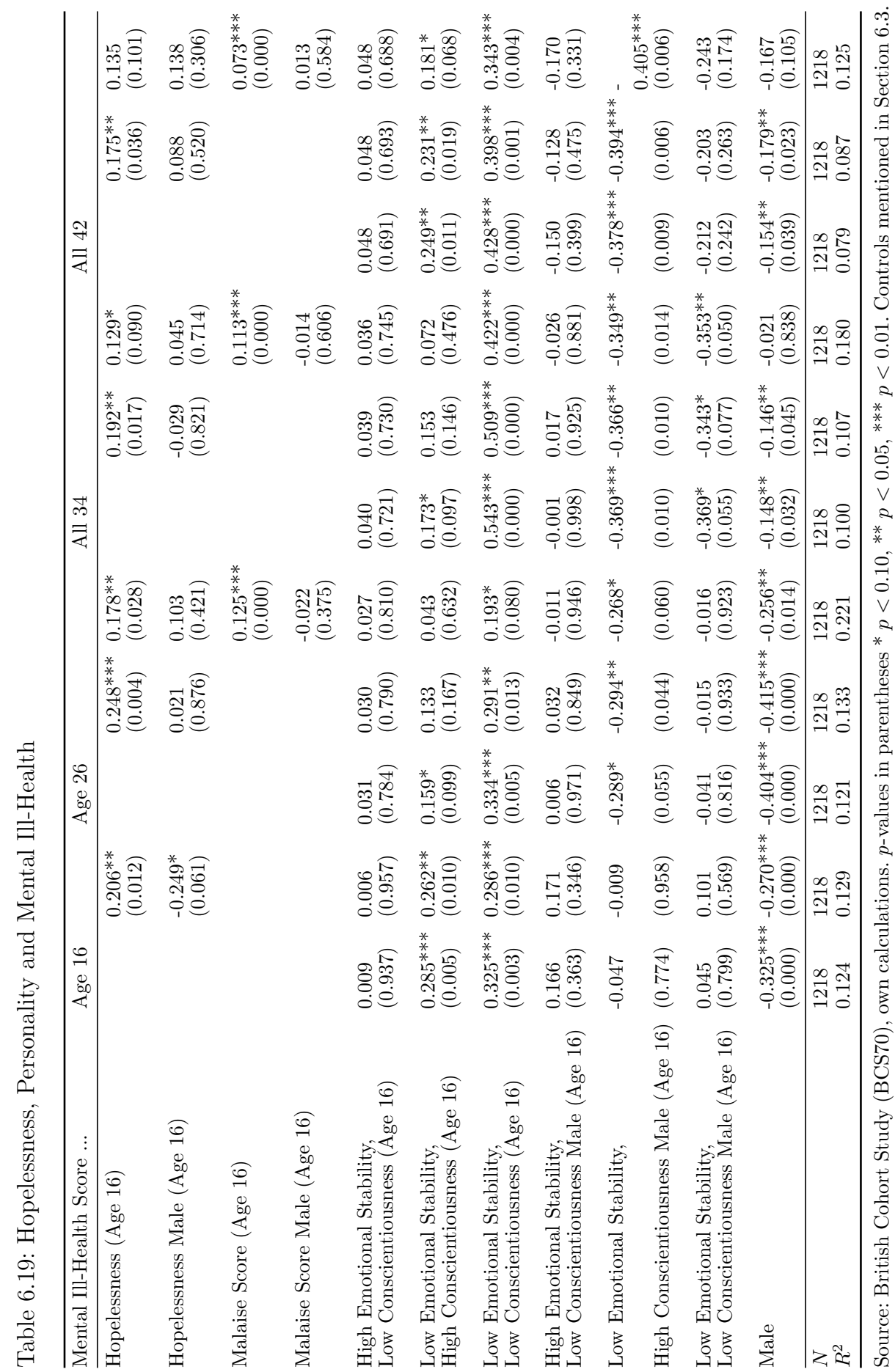


Table 6.20: Interaction between Helplessness and Personality

\begin{tabular}{|c|c|c|c|c|c|c|c|}
\hline Mental Ill-Health Score ... & Age 16 & \multicolumn{2}{|l|}{ Age 26} & \multicolumn{2}{|l|}{ All 34} & \multicolumn{2}{|l|}{ All 42} \\
\hline $\begin{array}{l}\text { High Emotional Stability, } \\
\text { High Conscientiousness } \\
\text { Help (Age 16) }\end{array}$ & $\begin{array}{l}-0.945^{* * *} \\
(0.000)\end{array}$ & $\begin{array}{l}-0.633^{* *} \\
(0.000)\end{array}$ & $\begin{array}{c}-0.337^{* * *} \\
(0.032)\end{array}$ & $\begin{array}{l}-0.871^{* *} \\
(0.000)\end{array}$ & $\begin{array}{l}-0.610^{* * *} \\
(0.000)\end{array}$ & $\begin{array}{l}-0.667^{* *} \\
(0.000)\end{array}$ & $\begin{array}{c}*-0.488^{* * *} \\
(0.004)\end{array}$ \\
\hline $\begin{array}{l}\text { High Emotional Stability, } \\
\text { High Conscientiousness } \\
\text { Helpless (Age 16) }\end{array}$ & $\begin{array}{l}-0.402^{* * *} \\
(0.007)\end{array}$ & $\begin{array}{c}-0.327^{* *} \\
(0.045)\end{array}$ & $\begin{array}{l}-0.202 \\
(0.189)\end{array}$ & $\begin{array}{l}-0.725^{* *} \\
(0.000)\end{array}$ & $\begin{array}{l}-0.614^{* * *} \\
(0.000)\end{array}$ & $\begin{array}{c}-0.516^{* *} \\
(0.003)\end{array}$ & $\begin{array}{c}*-0.439 * * * \\
(0.009)\end{array}$ \\
\hline $\begin{array}{l}\text { High Emotional Stability, } \\
\text { Low Conscientiousness } \\
\text { Help (Age 16) }\end{array}$ & $\begin{array}{l}-1.126^{* * *} \\
(0.000)\end{array}$ & $\begin{array}{c}-0.552^{* * x} \\
(0.009)\end{array}$ & $\begin{array}{l}-0.199 \\
(0.330)\end{array}$ & $\begin{array}{l}-0.843^{* *} \\
(0.000)\end{array}$ & $\begin{array}{l}-0.531^{* * *} \\
(0.006)\end{array}$ & $\begin{array}{c}-0.509^{* *} \\
(0.031)\end{array}$ & $\begin{array}{c}-0.296 \\
(0.199)\end{array}$ \\
\hline $\begin{array}{l}\text { High Emotional Stability, } \\
\text { Low Conscientiousness } \\
\text { Helpless (Age 16) }\end{array}$ & $\begin{array}{l}-0.297 \\
(0.104)\end{array}$ & $\begin{array}{c}-0.347^{*} \\
(0.059)\end{array}$ & $\begin{array}{l}-0.254 \\
(0.162)\end{array}$ & $\begin{array}{l}-0.682^{* *} \\
(0.002)\end{array}$ & $\begin{array}{l}-0.599 * * * \\
(0.006)\end{array}$ & $\begin{array}{c}-0.564^{* *} \\
(0.006)\end{array}$ & $\begin{array}{c}*-0.508^{* *} \\
(0.011)\end{array}$ \\
\hline $\begin{array}{l}\text { Low Emotional Stability, } \\
\text { High Conscientiousness } \\
\text { Help (Age 16) }\end{array}$ & $\begin{array}{l}-0.997^{* * *} \\
(0.000)\end{array}$ & $\begin{array}{l}-0.603^{* *} \\
(0.000)\end{array}$ & $\begin{array}{l}-0.291^{*} \\
(0.065)\end{array}$ & $\begin{array}{l}-0.870^{* *} \\
(0.000)\end{array}$ & $\begin{array}{l}-0.594^{* * *} \\
(0.003)\end{array}$ & $\begin{array}{l}-0.568^{* *} * \\
(0.004)\end{array}$ & $\begin{array}{c}*-0.380 * * \\
(0.049)\end{array}$ \\
\hline $\begin{array}{l}\text { Low Emotional Stability, } \\
\text { High Conscientiousness } \\
\text { Helpless (Age 16) }\end{array}$ & $\begin{array}{l}0.083 \\
(0.627)\end{array}$ & $\begin{array}{c}-0.081 \\
(0.651)\end{array}$ & $\begin{array}{l}-0.107 \\
(0.520)\end{array}$ & $\begin{array}{c}-0.432^{* *} \\
(0.027)\end{array}$ & $\begin{array}{l}-0.455^{* *} \\
(0.013)\end{array}$ & $\begin{array}{l}-0.185 \\
(0.305)\end{array}$ & $\begin{array}{c}-0.201 \\
(0.259)\end{array}$ \\
\hline $\begin{array}{l}\text { Low Emotional Stability, } \\
\text { Low Conscientiousness } \\
\text { Help (Age 16) }\end{array}$ & $\begin{array}{l}-0.819^{* * *} \\
(0.000)\end{array}$ & $\begin{array}{l}-0.316 \\
(0.135)\end{array}$ & $\begin{array}{l}-0.059 \\
(0.762)\end{array}$ & $\begin{array}{l}-0.669^{* *} \\
(0.001)\end{array}$ & $\begin{array}{l}-0.443^{* *} \\
(0.018)\end{array}$ & $\begin{array}{c}-0.488^{* *} \\
(0.013)\end{array}$ & $\begin{array}{c}-0.333^{*} \\
(0.085)\end{array}$ \\
\hline $\begin{array}{l}\text { High Emotional Stability, } \\
\text { High Conscientiousness } \\
\text { Help Male (Age 16) }\end{array}$ & $\begin{array}{l}0.048 \\
(0.830)\end{array}$ & $\begin{array}{l}0.192 \\
(0.439)\end{array}$ & $\begin{array}{l}0.177 \\
(0.444)\end{array}$ & $\begin{array}{l}0.434^{*} \\
(0.091)\end{array}$ & $\begin{array}{l}0.421^{*} \\
(0.088)\end{array}$ & $\begin{array}{l}0.068 \\
(0.792)\end{array}$ & $\begin{array}{l}0.059 \\
(0.818)\end{array}$ \\
\hline $\begin{array}{l}\text { High Emotional Stability, } \\
\text { High Conscientiousness } \\
\text { Helpless Male (Age 16) }\end{array}$ & $\begin{array}{l}0.072 \\
(0.761)\end{array}$ & $\begin{array}{l}-0.062 \\
(0.806)\end{array}$ & $\begin{array}{c}-0.084 \\
(0.716)\end{array}$ & $\begin{array}{l}0.441^{*} \\
(0.098)\end{array}$ & $\begin{array}{l}0.421^{*} \\
(0.099)\end{array}$ & $\begin{array}{l}0.155 \\
(0.561)\end{array}$ & $\begin{array}{l}0.141 \\
(0.591)\end{array}$ \\
\hline $\begin{array}{l}\text { High Emotional Stability, } \\
\text { Low Conscientiousness } \\
\text { Help Male (Age 16) }\end{array}$ & $\begin{array}{l}0.418 \\
(0.178)\end{array}$ & $\begin{array}{l}0.209 \\
(0.537)\end{array}$ & $\begin{array}{l}0.078 \\
(0.811)\end{array}$ & $\begin{array}{l}0.546 \\
(0.117)\end{array}$ & $\begin{array}{l}0.431 \\
(0.197)\end{array}$ & $\begin{array}{c}-0.054 \\
(0.877)\end{array}$ & $\begin{array}{c}-0.134 \\
(0.697)\end{array}$ \\
\hline $\begin{array}{l}\text { High Emotional Stability, } \\
\text { Low Conscientiousness } \\
\text { Helpless Male (Age 16) }\end{array}$ & $\begin{array}{l}0.071 \\
(0.809)\end{array}$ & $\begin{array}{l}-0.069 \\
(0.802)\end{array}$ & $\begin{array}{l}-0.091 \\
(0.725)\end{array}$ & $\begin{array}{l}0.355 \\
(0.263)\end{array}$ & $\begin{array}{l}0.335 \\
(0.266)\end{array}$ & $\begin{array}{l}0.001 \\
(0.996)\end{array}$ & $\begin{array}{l}-0.012 \\
(0.967)\end{array}$ \\
\hline $\begin{array}{l}\text { Low Emotional Stability, } \\
\text { High Conscientiousness } \\
\text { Help Male (Age 16) }\end{array}$ & $\begin{array}{l}0.153 \\
(0.571)\end{array}$ & $\begin{array}{l}0.153 \\
(0.602)\end{array}$ & $\begin{array}{l}0.105 \\
(0.707)\end{array}$ & $\begin{array}{l}0.272 \\
(0.382)\end{array}$ & $\begin{array}{l}0.229 \\
(0.448)\end{array}$ & $\begin{array}{l}0.059 \\
(0.849)\end{array}$ & $\begin{array}{l}0.030 \\
(0.923)\end{array}$ \\
\hline $\begin{array}{l}\text { Low Emotional Stability, } \\
\text { High Conscientiousness }\end{array}$ & $\begin{array}{l}-0.159 \\
(0.560)\end{array}$ & $\begin{array}{l}-0.511^{*} \\
(0.067)\end{array}$ & $\begin{array}{l}-0.461^{*} \\
(0.079)\end{array}$ & $\begin{array}{l}-0.040 \\
(0.886)\end{array}$ & $\begin{array}{l}0.004 \\
(0.989)\end{array}$ & $\begin{array}{l}-0.392 \\
(0.150)\end{array}$ & $\begin{array}{l}-0.362 \\
(0.178)\end{array}$ \\
\hline
\end{tabular}


... Table 6.20 continued

\begin{tabular}{llllllll}
\hline Mental Ill-Health Score $\ldots$ & Age 16 & Age 26 & \multicolumn{3}{c}{ All 34} & & All 42 \\
\hline Helpless Male (Age 16) & & & & & & & \\
Low Emotional Stability, & 0.118 & -0.079 & -0.116 & 0.328 & 0.295 & -0.017 & -0.040 \\
Low Conscientiousness & $(0.700)$ & $(0.797)$ & $(0.702)$ & $(0.332)$ & $(0.363)$ & $(0.952)$ & $(0.892)$ \\
Help Male (Age 16) & & & & & & & \\
Malaise Score & & & $0.117^{* * *}$ & & $0.103^{* * *}$ & & $0.071^{* * *}$ \\
(Age 16) & & & $(0.000)$ & & $(0.000)$ & & $(0.000)$ \\
& & & & & & & \\
Male & $-0.377^{*}$ & $-0.462^{* *}$ & $-0.344^{*}$ & $-0.603^{* *}$ & $-0.499^{* *}$ & -0.298 & -0.227 \\
& $(0.061)$ & $(0.033)$ & $(0.086)$ & $(0.010)$ & $(0.026)$ & $(0.193)$ & $(0.315)$ \\
\hline$N$ & & & & & & & \\
$R^{2}$ & 1218 & 1218 & 1218 & 1218 & 1218 & 1218 & 1218 \\
\hline
\end{tabular}

Source: British Cohort Study (BCS70), own calculations.

Note: $p$-values in parentheses ${ }^{*} p<0.10,{ }^{* *} p<0.05$, *** $p<0.01$. The OLS regressions include controls for mother's age, mother's age squared, region of living and parental socioeconomic background at birth, the personality traits agreeableness and extraversion measured at the age of 16 as well as cognitive ability measured at the age of 10 .

Table 6.21: Interaction between Hopelessness and Personality

\begin{tabular}{|c|c|c|c|c|c|c|}
\hline Mental Ill-Health Score ... & Age 16 & Age 26 & & All 34 & All 42 & \\
\hline $\begin{array}{l}\text { High Emotional Stability, } \\
\text { High Conscientiousness } \\
\text { Hope (Age 16) }\end{array}$ & $\begin{array}{l}-0.473^{* * *} \\
(0.005)\end{array}$ & $\begin{array}{l}{ }^{k}-0.665^{* * * *} \\
(0.000)\end{array}$ & $\begin{array}{l}-0.517^{* * * *} \\
(0.003)\end{array}$ & $\begin{array}{l}-0.896^{* * *}-0.761^{* * *} \\
(0.000) \quad(0.000)\end{array}$ & $\begin{array}{l}*-0.698^{* * *} \\
(0.000)\end{array}$ & $\begin{array}{l}-0.602^{* * * *} \\
(0.001)\end{array}$ \\
\hline $\begin{array}{l}\text { High Emotional Stability, } \\
\text { High Conscientiousness } \\
\text { Hopeless (Age 16) }\end{array}$ & $\begin{array}{l}-0.264 \\
(0.164)\end{array}$ & $\begin{array}{l}-0.569^{* * *} \\
(0.005)\end{array}$ & $\begin{array}{l}-0.486^{* *} \\
(0.012)\end{array}$ & $\begin{array}{ll}-0.871^{* * *}-0.795^{* * *} \\
(0.000) & (0.000)\end{array}$ & $\begin{array}{l}{ }^{*}-0.551^{* * *} \\
(0.006)\end{array}$ & $\begin{array}{l}-0.498^{* *} \\
(0.012)\end{array}$ \\
\hline $\begin{array}{l}\text { High Emotional Stability, } \\
\text { Low Conscientiousness } \\
\text { Hope (Age 16) }\end{array}$ & $\begin{array}{l}-0.489^{* *} \\
(0.010)\end{array}$ & $\begin{array}{l}-0.649^{* * *} \\
(0.001)\end{array}$ & $\begin{array}{l}-0.495^{* * * *} \\
(0.008)\end{array}$ & $\begin{array}{l}-0.853^{* * *}-0.713^{* * *} \\
(0.000) \quad(0.000)\end{array}$ & $\begin{array}{l}{ }^{*}-0.610^{* * * *} \\
(0.003)\end{array}$ & $\begin{array}{l}-0.511^{* *} \\
(0.011)\end{array}$ \\
\hline $\begin{array}{l}\text { High Emotional Stability, } \\
\text { Low Conscientiousness } \\
\text { Hopeless (Age 16) }\end{array}$ & $\begin{array}{l}-0.224 \\
(0.413)\end{array}$ & $\begin{array}{l}-0.448 \\
(0.122)\end{array}$ & $\begin{array}{l}-0.378 \\
(0.184)\end{array}$ & $\begin{array}{l}-0.814^{* * *}-0.750^{* * *} \\
(0.004) \quad(0.005)\end{array}$ & $\begin{array}{l}-0.637^{* *} \\
(0.035)\end{array}$ & $\begin{array}{l}-0.591^{* *} \\
(0.046)\end{array}$ \\
\hline $\begin{array}{l}\text { Low Emotional Stability, } \\
\text { High Conscientiousness } \\
\text { Hope (Age 16) }\end{array}$ & $\begin{array}{l}-0.199 \\
(0.294)\end{array}$ & $\begin{array}{l}-0.583^{* * *} \\
(0.002)\end{array}$ & $\begin{array}{l}-0.521^{* * * *} \\
(0.003)\end{array}$ & $\begin{array}{l}-0.765^{* * *}-0.708^{* * *} \\
(0.000) \quad(0.000)\end{array}$ & $\begin{array}{l}{ }^{*}-0.423^{* *} \\
(0.032)\end{array}$ & $\begin{array}{l}-0.382^{* *} \\
(0.049)\end{array}$ \\
\hline $\begin{array}{l}\text { Low Emotional Stability, } \\
\text { High Conscientiousness } \\
\text { Hopeless (Age 16) }\end{array}$ & $\begin{array}{l}-0.059 \\
(0.781)\end{array}$ & $\begin{array}{l}-0.253 \\
(0.252)\end{array}$ & $\begin{array}{l}-0.235 \\
(0.238)\end{array}$ & $\begin{array}{l}-0.610^{* * *}-0.594^{* * *} \\
(0.006) \quad(0.003)\end{array}$ & $\begin{array}{l}-0.418^{* *} \\
(0.037)\end{array}$ & $\begin{array}{l}-0.406^{* *} \\
(0.038)\end{array}$ \\
\hline
\end{tabular}


... Table 6.21 continued

\begin{tabular}{|c|c|c|c|c|c|c|c|}
\hline Mental Ill-Health Score ... & Age 16 & Age 26 & & All 34 & & All 42 & \\
\hline $\begin{array}{l}\text { Low Emotional Stability, } \\
\text { Low Conscientiousness } \\
\text { Hope (Age 16) }\end{array}$ & $\begin{array}{l}-0.226 \\
(0.249)\end{array}$ & $\begin{array}{l}-0.533^{* * * *} \\
(0.009)\end{array}$ & $\begin{array}{l}-0.462^{* *} \\
(0.015)\end{array}$ & $\begin{array}{l}-0.676^{* * *} \\
(0.002)\end{array}$ & $\begin{array}{l}-0.611^{* * * *} \\
(0.002)\end{array}$ & $\begin{array}{l}-0.510^{* *} \\
(0.012)\end{array}$ & $\begin{array}{l}-0.464^{* *} \\
(0.019)\end{array}$ \\
\hline $\begin{array}{l}\text { High Emotional Stability, } \\
\text { High Conscientiousness } \\
\text { Hope Male (Age 16) }\end{array}$ & $\begin{array}{l}0.097 \\
(0.732)\end{array}$ & $\begin{array}{l}0.441^{*} \\
(0.089)\end{array}$ & $\begin{array}{l}0.410^{*} \\
(0.096)\end{array}$ & $\begin{array}{l}0.566^{* *} \\
(0.044)\end{array}$ & $\begin{array}{l}0.538 * * \\
(0.048)\end{array}$ & $\begin{array}{l}0.216 \\
(0.476)\end{array}$ & $\begin{array}{l}0.196 \\
(0.519)\end{array}$ \\
\hline $\begin{array}{l}\text { High Emotional Stability, } \\
\text { High Conscientiousness } \\
\text { Hopeless Male (Age 16) }\end{array}$ & $\begin{array}{l}-0.096 \\
(0.758)\end{array}$ & $\begin{array}{l}0.710^{* *} \\
(0.016)\end{array}$ & $\begin{array}{l}0.740 * * * \\
(0.008)\end{array}$ & $\begin{array}{l}0.803^{* * *} \\
(0.009)\end{array}$ & $\begin{array}{l}0.831^{* * * *} \\
(0.006)\end{array}$ & $\begin{array}{l}0.368 \\
(0.269)\end{array}$ & $\begin{array}{l}0.388 \\
(0.242)\end{array}$ \\
\hline $\begin{array}{l}\text { High Emotional Stability, } \\
\text { Low Conscientiousness } \\
\text { Hope Male (Age 16) }\end{array}$ & $\begin{array}{l}0.372 \\
(0.251)\end{array}$ & $\begin{array}{l}0.533^{*} \\
(0.055)\end{array}$ & $\begin{array}{l}0.416 \\
(0.122)\end{array}$ & $\begin{array}{l}0.698^{* *} \\
(0.031)\end{array}$ & $\begin{array}{l}0.591^{*} \\
(0.059)\end{array}$ & $\begin{array}{l}0.118 \\
(0.715)\end{array}$ & $\begin{array}{l}0.042 \\
(0.895)\end{array}$ \\
\hline $\begin{array}{l}\text { High Emotional Stability, } \\
\text { Low Conscientiousness } \\
\text { Hopeless Male (Age 16) }\end{array}$ & $\begin{array}{l}-0.216 \\
(0.574)\end{array}$ & $\begin{array}{l}0.459 \\
(0.338)\end{array}$ & $\begin{array}{l}0.526 \\
(0.269)\end{array}$ & $\begin{array}{l}0.364 \\
(0.341)\end{array}$ & $\begin{array}{l}0.425 \\
(0.254)\end{array}$ & $\begin{array}{l}0.215 \\
(0.694)\end{array}$ & $\begin{array}{l}0.259 \\
(0.636)\end{array}$ \\
\hline $\begin{array}{l}\text { Low Emotional Stability, } \\
\text { High Conscientiousness } \\
\text { Hope Male (Age 16) }\end{array}$ & $\begin{array}{l}0.080 \\
(0.803)\end{array}$ & $\begin{array}{l}0.044 \\
(0.867)\end{array}$ & $\begin{array}{l}0.019 \\
(0.941)\end{array}$ & $\begin{array}{l}0.185 \\
(0.532)\end{array}$ & $\begin{array}{l}0.162 \\
(0.576)\end{array}$ & $\begin{array}{l}-0.266 \\
(0.394)\end{array}$ & $\begin{array}{l}-0.282 \\
(0.369)\end{array}$ \\
\hline $\begin{array}{l}\text { Low Emotional Stability, } \\
\text { High Conscientiousness } \\
\text { Hope Male (Age 16) }\end{array}$ & $\begin{array}{l}0.019 \\
(0.956)\end{array}$ & $\begin{array}{l}0.440 \\
(0.182)\end{array}$ & $\begin{array}{l}0.434 \\
(0.172)\end{array}$ & $\begin{array}{l}0.454 \\
(0.173)\end{array}$ & $\begin{array}{l}0.449 \\
(0.164)\end{array}$ & $\begin{array}{l}0.283 \\
(0.415)\end{array}$ & $\begin{array}{l}0.279 \\
(0.421)\end{array}$ \\
\hline $\begin{array}{l}\text { Low Emotional Stability, } \\
\text { Low Conscientiousness } \\
\text { Hope Male (Age 16) }\end{array}$ & $\begin{array}{l}0.358 \\
(0.271)\end{array}$ & $\begin{array}{l}0.843^{* * *} \\
(0.007)\end{array}$ & $\begin{array}{l}0.730 * * \\
(0.013)\end{array}$ & $\begin{array}{l}0.715^{* *} \\
(0.035)\end{array}$ & $\begin{array}{l}0.613^{*} \\
(0.058)\end{array}$ & $\begin{array}{l}0.437 \\
(0.192)\end{array}$ & $\begin{array}{l}0.364 \\
(0.272)\end{array}$ \\
\hline $\begin{array}{l}\text { Malaise Score } \\
\text { (Age 16) }\end{array}$ & & & $\begin{array}{l}0.117^{* * *} \\
(0.000)\end{array}$ & & $\begin{array}{l}0.107^{* * *} \\
(0.000)\end{array}$ & & $\begin{array}{l}0.076 \text { *** } \\
(0.000)\end{array}$ \\
\hline Male & $\begin{array}{l}-0.410 \\
(0.114)\end{array}$ & $\begin{array}{l}-0.910^{* * *} \\
(0.000)\end{array}$ & $\begin{array}{l}-0.781^{* * * *} \\
(0.001)\end{array}$ & $\begin{array}{c}{ }^{k}-0.800^{* * *} \\
(0.002)\end{array}$ & $\begin{array}{l}-0.683^{* * * *} \\
(0.007)\end{array}$ & $\begin{array}{l}{ }^{k}-0.456 \\
(0.104)\end{array}$ & $\begin{array}{l}-0.372 \\
(0.185)\end{array}$ \\
\hline $\begin{array}{l}N \\
R^{2}\end{array}$ & $\begin{array}{l}1218 \\
0.132\end{array}$ & $\begin{array}{l}1218 \\
0.146\end{array}$ & $\begin{array}{l}1218 \\
0.231\end{array}$ & $\begin{array}{l}1218 \\
0.125\end{array}$ & $\begin{array}{l}1218 \\
0.196\end{array}$ & $\begin{array}{l}1218 \\
0.102\end{array}$ & $\begin{array}{l}1218 \\
0.138\end{array}$ \\
\hline
\end{tabular}

Source: British Cohort Study (BCS70), own calculations.

Note: $p$-values in parentheses ${ }^{*} p<0.10,{ }^{* *} p<0.05,{ }^{* * *} p<0.01$. The OLS regressions include controls for mother's age, mother's age squared, region of living and parental socioeconomic background at birth, the personality traits agreeableness and extraversion measured at the age of 16 as well as cognitive ability measured at the age of 10 . 
Table 6.22: Helplessness Change, Gender and Mental Ill-Health

\begin{tabular}{|c|c|c|c|c|c|c|}
\hline Mental Ill-Health Score ... & Age 30 & & Age 34 & & All 42 & \\
\hline $\begin{array}{l}\text { Helplessness } \\
\text { (Age 16) }\end{array}$ & $\begin{array}{l}0.420 * * * \\
(0.000)\end{array}$ & $\begin{array}{l}0.316^{* * * *} \\
(0.000)\end{array}$ & $\begin{array}{l}0.356^{* * *} \\
(0.000)\end{array}$ & $\begin{array}{l}0.246^{* * *} \\
(0.000)\end{array}$ & $\begin{array}{l}0.269^{* * *} \\
(0.000)\end{array}$ & $\begin{array}{l}0.194^{* * * *} \\
(0.000)\end{array}$ \\
\hline $\begin{array}{l}\text { Helplessness Change } \\
(\text { Age } 30-16)>1\end{array}$ & $\begin{array}{l}0.551^{* * *} \\
(0.000)\end{array}$ & $\begin{array}{l}0.530 * * * \\
(0.000)\end{array}$ & $\begin{array}{l}0.279^{* * *} \\
(0.001)\end{array}$ & $\begin{array}{l}0.257^{* * *} \\
(0.002)\end{array}$ & $\begin{array}{l}0.312^{* * *} \\
(0.000)\end{array}$ & $\begin{array}{l}0.297 * * * \\
(0.001)\end{array}$ \\
\hline $\begin{array}{l}\text { Helplessness Change } \\
\text { (Age } 30-16)<-1\end{array}$ & $\begin{array}{l}-0.577^{* * *} \\
(0.000)\end{array}$ & $\begin{array}{l}-0.531^{* * *} \\
(0.000)\end{array}$ & $\begin{array}{l}-0.507^{* * *} \\
(0.000)\end{array}$ & $\begin{array}{l}-0.458^{* * *} \\
(0.000)\end{array}$ & $\begin{array}{l}-0.304^{* * *} \\
(0.002)\end{array}$ & $\begin{array}{l}-0.271^{* * *} \\
(0.005)\end{array}$ \\
\hline $\begin{array}{l}\text { Helplessness Change } \\
\text { Male (Age 30-16) }>1\end{array}$ & $\begin{array}{l}0.103 \\
(0.452)\end{array}$ & $\begin{array}{l}0.076 \\
(0.568)\end{array}$ & $\begin{array}{l}0.057 \\
(0.670)\end{array}$ & $\begin{array}{l}0.029 \\
(0.828)\end{array}$ & $\begin{array}{l}0.075 \\
(0.602)\end{array}$ & $\begin{array}{l}0.056 \\
(0.699)\end{array}$ \\
\hline $\begin{array}{l}\text { Helplessness Change } \\
\text { Male (Age 30-16) }<-1\end{array}$ & $\begin{array}{l}-0.092 \\
(0.425)\end{array}$ & $\begin{array}{l}-0.043 \\
(0.706)\end{array}$ & $\begin{array}{l}-0.050 \\
(0.693)\end{array}$ & $\begin{array}{l}0.002 \\
(0.986)\end{array}$ & $\begin{array}{l}-0.113 \\
(0.362)\end{array}$ & $\begin{array}{l}-0.078 \\
(0.529)\end{array}$ \\
\hline $\begin{array}{l}\text { Emotional Stability } \\
\text { (Age 16) }\end{array}$ & $\begin{array}{l}-0.118^{* * *} \\
(0.001)\end{array}$ & $\begin{array}{l}-0.084^{* *} \\
(0.014)\end{array}$ & $\begin{array}{l}-0.134^{* * *} \\
(0.000)\end{array}$ & $\begin{array}{l}-0.098^{* * *} \\
(0.007)\end{array}$ & $\begin{array}{l}-0.163^{* * *} \\
(0.000)\end{array}$ & $\begin{array}{l}-0.139 * * * \\
(0.000)\end{array}$ \\
\hline $\begin{array}{l}\text { Conscientiousness } \\
\text { (Age 16) }\end{array}$ & $\begin{array}{l}-0.045 \\
(0.170)\end{array}$ & $\begin{array}{l}-0.037 \\
(0.232)\end{array}$ & $\begin{array}{l}-0.057 \\
(0.108)\end{array}$ & $\begin{array}{l}-0.049 \\
(0.142)\end{array}$ & $\begin{array}{l}-0.010 \\
(0.763)\end{array}$ & $\begin{array}{l}-0.005 \\
(0.884)\end{array}$ \\
\hline $\begin{array}{l}\text { Malaise Score } \\
\text { (Age 16) }\end{array}$ & & $\begin{array}{l}0.081^{* * * *} \\
(0.000)\end{array}$ & & $\begin{array}{l}0.085^{* * *} \\
(0.000)\end{array}$ & & $\begin{array}{l}0.058^{* * * *} \\
(0.000)\end{array}$ \\
\hline Male & $\begin{array}{l}-0.307^{* * *} \\
(0.000)\end{array}$ & $\begin{array}{l}-0.254^{* * *} \\
(0.000)\end{array}$ & $\begin{array}{l}-0.243^{* * *} \\
(0.003)\end{array}$ & $\begin{array}{l}-0.187^{* *} \\
(0.021)\end{array}$ & $\begin{array}{l}-0.229^{* * *} \\
(0.005)\end{array}$ & $\begin{array}{l}-0.191^{* *} \\
(0.017)\end{array}$ \\
\hline$N$ & 1218 & 1218 & 1218 & 1218 & 1218 & 1218 \\
\hline$R^{2}$ & 0.230 & 0.263 & 0.174 & 0.211 & 0.131 & 0.148 \\
\hline
\end{tabular}

Source: British Cohort Study (BCS70), own calculations.

Note: $p$-values in parentheses ${ }^{*} p<0.10,{ }^{* *} p<0.05, * * * p<0.01$. The OLS regressions include controls for mother's age, mother's age squared, region of living and parental socioeconomic background at birth, the personality traits agreeableness and extraversion measured at the age of 16 as well as cognitive ability measured at the age of 10 . 
Table 6.23: Helplessness Change, Personality and Mental Ill-Health

\begin{tabular}{lllllll}
\hline Mental Ill-Health Score $\ldots$ & Age 30 & & Age 34 & \multicolumn{2}{c}{ All 42} \\
\hline Helplessness (Age 16) & $0.433^{* * *}$ & $0.313^{* * *}$ & $0.367^{* * *}$ & $0.243^{* * *}$ & $0.279^{* * *}$ & $0.188^{* * *}$ \\
& $(0.000)$ & $(0.000)$ & $(0.000)$ & $(0.000)$ & $(0.000)$ & $(0.000)$
\end{tabular}

High Emotional Stability, Low Conscientiousness (Age 16)

Low Emotional Stability, High Conscientiousness (Age 16)

Low Emotional Stability, Low Conscientiousness (Age 16)

High Emotional Stability, High Conscientiousness Helplessness Change (Age 30-16) > 1

High Emotional Stability, High Conscientiousness Helplessness Change (Age 30-16) $<-1$

High Emotional Stability, Low Conscientiousness Helplessness Change (Age 30-16) > 1

High Emotional Stability, Low Conscientiousness Helplessness Change (Age 30-16) $<-1$

Low Emotional Stability, High Conscientiousness Helplessness Change (Age 30-16) > 1

Low Emotional Stability, High Conscientiousness Helplessness Change (Age 30-16) $<-1$

$\begin{array}{llllll}0.096 & 0.087 & 0.018 & 0.009 & -0.030 & -0.037 \\ (0.381) & (0.417) & (0.885) & (0.942) & (0.807) & (0.760)\end{array}$

$\begin{array}{llllll}0.139 & 0.090 & 0.120 & 0.069 & 0.217^{*} & 0.181 \\ (0.193) & (0.389) & (0.301) & (0.533) & (0.058) & (0.111)\end{array}$

$\begin{array}{llllll}0.251^{*} & 0.209^{*} & 0.404^{* * *} & 0.361^{* * *} & 0.416^{* * *} & 0.384^{* * *} \\ (0.052) & (0.090) & (0.004) & (0.008) & (0.003) & (0.005)\end{array}$

$\begin{array}{llllll}0.590^{* * *} & 0.557^{* * *} & 0.337^{* * *} & 0.302^{* * *} & 0.417^{* * *} & 0.392^{* * *} \\ (0.000) & (0.000) & (0.000) & (0.000) & (0.000) & (0.000)\end{array}$

$\begin{array}{llllll}-0.617^{* * * *} & -0.522^{* * *} & -0.514^{* * *} & -0.416^{* * *} & -0.319^{* * *} & -0.248^{* *} \\ (0.000) & (0.000) & (0.000) & (0.000) & (0.002) & (0.014)\end{array}$

$\begin{array}{llllll}0.469^{* *} & 0.471^{* *} & 0.224 & 0.226 & 0.285 & 0.286 \\ (0.018) & (0.018) & (0.253) & (0.235) & (0.188) & (0.174)\end{array}$

$\begin{array}{cccccc}-0.344^{*} & -0.308 & -0.356^{*} & -0.320^{*} & -0.143 & -0.117 \\ (0.076) & (0.103) & (0.066) & (0.094) & (0.476) & (0.545)\end{array}$

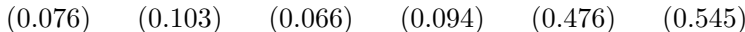

$\begin{array}{llllll}0.411^{* *} & 0.394^{* *} & 0.073 & 0.056 & 0.109 & 0.097 \\ (0.012) & (0.016) & (0.704) & (0.773) & (0.524) & (0.573)\end{array}$

$$
\begin{array}{llllll}
-0.624^{* * *} & -0.576^{* * *} & -0.526^{* * *} & -0.476^{* * *} & -0.350^{* *} & -0.313^{* *} \\
(0.000) & (0.000) & (0.000) & (0.001) & (0.022) & (0.040)
\end{array}
$$


... Table 6.23 continued

\begin{tabular}{|c|c|c|c|c|c|c|}
\hline Mental Ill-Health Score ... & Age 30 & & Age 34 & & All 42 & \\
\hline $\begin{array}{l}\text { Low Emotional Stability, } \\
\text { Low Conscientiousness } \\
\text { Helplessness Change } \\
(\text { Age } 30-16)>1\end{array}$ & $\begin{array}{l}0.851^{* * *} \\
(0.000)\end{array}$ & $\begin{array}{l}0.762^{* * *} \\
(0.000)\end{array}$ & $\begin{array}{l}0.419^{* *} \\
(0.035)\end{array}$ & $\begin{array}{l}0.327^{*} \\
(0.088)\end{array}$ & $\begin{array}{l}0.323^{*} \\
(0.096)\end{array}$ & $\begin{array}{l}0.256 \\
(0.193)\end{array}$ \\
\hline $\begin{array}{l}\text { Low Emotional Stability, } \\
\text { Low Conscientiousness } \\
\text { Helplessness Change } \\
\text { (Age } 30-16)<-1\end{array}$ & $\begin{array}{l}-0.762^{* * *} \\
(0.000)\end{array}$ & $\begin{array}{l}-0.705^{* * *} \\
(0.000)\end{array}$ & $\begin{array}{l}-0.698^{* * *} \\
(0.000)\end{array}$ & $\begin{array}{l}-0.639^{* * *} \\
(0.001)\end{array}$ & $\begin{array}{l}-0.559^{* * *} \\
(0.002)\end{array}$ & $\begin{array}{l}-0.516^{* * *} \\
(0.004)\end{array}$ \\
\hline Malaise Score (Age 16) & & $\begin{array}{l}0.090^{* * *} \\
(0.000)\end{array}$ & & $\begin{array}{l}0.093^{* * *} \\
(0.000)\end{array}$ & & $\begin{array}{l}0.068^{* * *} * \\
(0.000)\end{array}$ \\
\hline$N$ & 1218 & 1218 & 1218 & 1218 & 1218 & 1218 \\
\hline$R^{2}$ & 0.211 & 0.254 & 0.163 & 0.209 & 0.115 & 0.139 \\
\hline
\end{tabular}

Source: British Cohort Study (BCS70), own calculations.

Note: $p$-values in parentheses ${ }^{*} p<0.10,{ }^{* *} p<0.05,{ }^{* * *} p<0.01$. The OLS regressions include controls for mother's age, mother's age squared, region of living and parental socioeconomic background at birth, the personality traits agreeableness and extraversion measured at the age of 16 as well as cognitive ability measured at the age of 10 . 


\subsubsection{Imputation of Missing Values}

Table 6.24: Personality Trait Change and Help- and Hopelessness

\begin{tabular}{|c|c|c|}
\hline Pessimistic Expectations (Age 16) ... & Helplessness & Hopelessness \\
\hline Emotional Stability (Age 10) & $-0.084^{* * *}(0.000)$ & $-0.074^{* * *}(0.000)$ \\
\hline Conscientiousness (Age 10) & $(0.562)$ & $(0.175)$ \\
\hline Emotional Stability Male (Age 10) & $0.086^{* * *}(0.006)$ & $(0.612)$ \\
\hline Conscientiousness Male (Age 10) & $(0.791)$ & $(0.238)$ \\
\hline Emotional Stability (Age 16-10) $>0.5$ & $-0.134^{* * *}(0.001)$ & $(0.345)$ \\
\hline Emotional Stability (Age 16-10) $<-0.5$ & $(0.383)$ & $0.112^{* * *}(0.000)$ \\
\hline Conscientiousness (Age 16-10) $>0.5$ & $(0.430)$ & $(0.601)$ \\
\hline Conscientiousness (Age 16-10) $<-0.5$ & $(0.870)$ & $(0.287)$ \\
\hline Emotional Stability Male (Age 16-10) $>0.5$ & $(0.057)$ & $(0.519)$ \\
\hline Emotional Stability Male (Age 16-10) $<-0.5$ & $(0.913)$ & $(0.391)$ \\
\hline Conscientiousness Male (Age 16-10) $>0.5$ & $(0.839)$ & $(0.772)$ \\
\hline Conscientiousness Male (Age 16-10) $<-0.5$ & $(0.649)$ & $(0.959)$ \\
\hline Male & $(0.195)$ & $(0.569)$ \\
\hline$N$ & 2309 & 2309 \\
\hline$R^{2}$ & 0.045 & 0.056 \\
\hline
\end{tabular}

Source: British Cohort Study (BCS70), own calculations.

Note: $p$-values in parentheses ${ }^{*} p<0.10,{ }^{* *} p<0.05,{ }^{* * *} p<0.01$. The OLS regressions include controls for mother's age, mother's age squared, region of living and parental socioeconomic background at birth, the personality traits agreeableness and extraversion measured at the ages of 10 and 16 as well as cognitive ability measured at the age of 10 . 


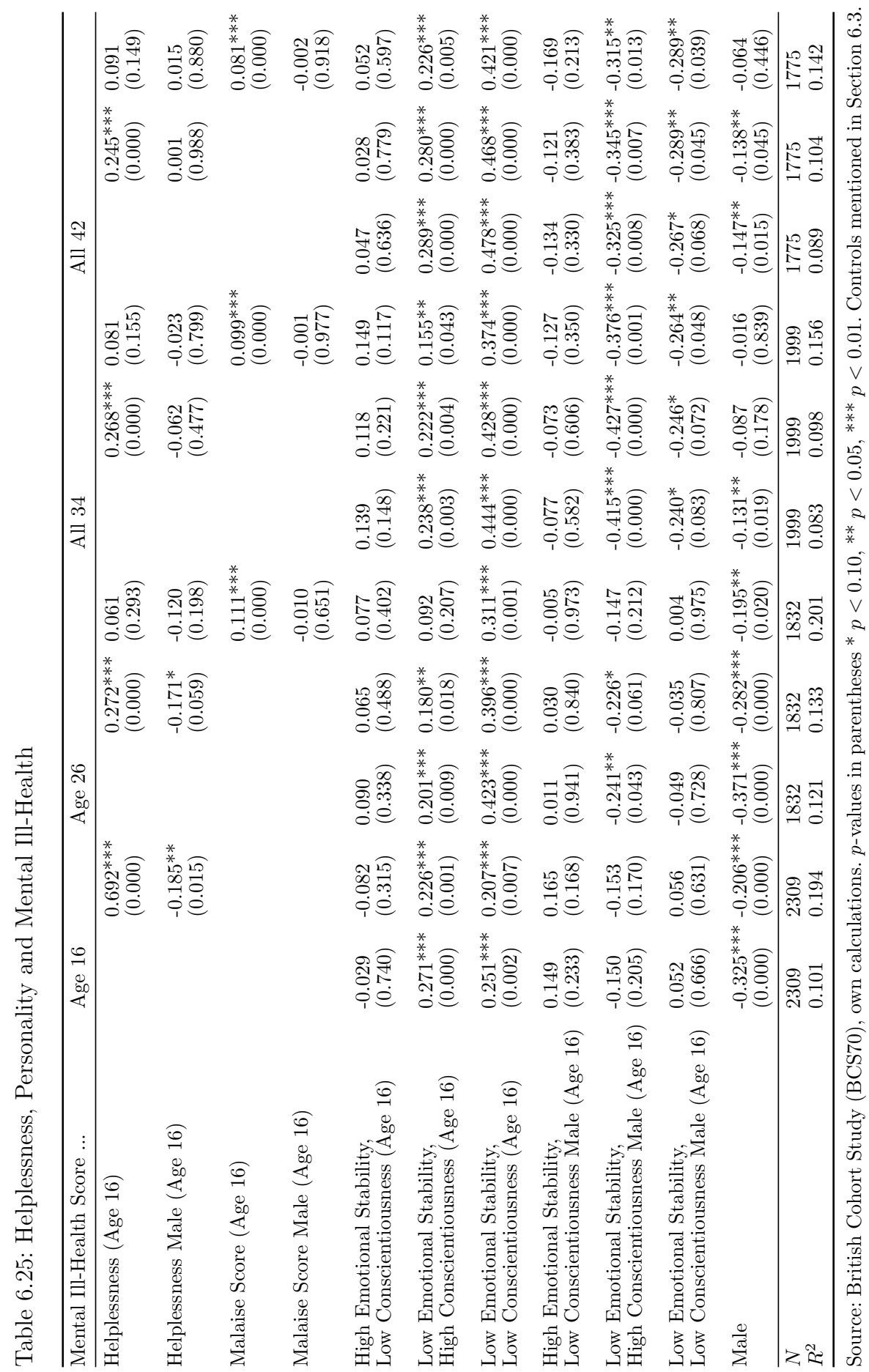




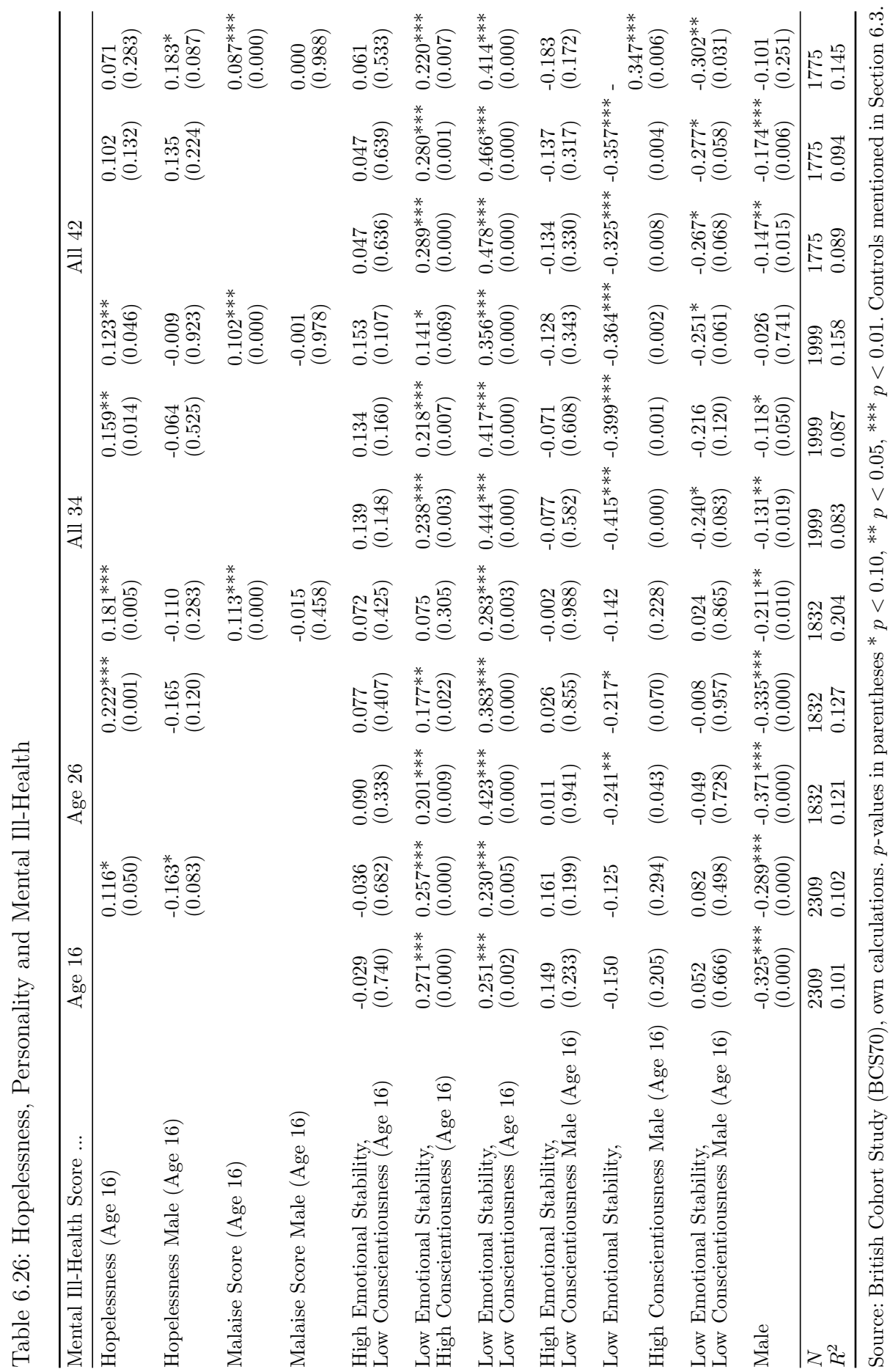


Table 6.27: Interaction between Helplessness and Personality

\begin{tabular}{|c|c|c|c|c|c|c|c|}
\hline Mental Ill-Health Score ... & Age 16 & Age 26 & & All 34 & & All 42 & \\
\hline $\begin{array}{l}\text { High Emotional Stability, } \\
\text { High Conscientiousness } \\
\text { Help (Age 16) }\end{array}$ & $\begin{array}{l}-0.867^{* * * *} \\
(0.000)\end{array}$ & $\begin{array}{l}-0.615^{* * * *} \\
(0.000)\end{array}$ & $\begin{array}{l}-0.329 \text { *** } \\
(0.008)\end{array}$ & $\begin{array}{l}-0.710^{* *} \\
(0.000)\end{array}$ & $\begin{array}{c}-0.483^{* * *} \\
(0.000)\end{array}$ & $\begin{array}{l}-0.777^{* * *} \\
(0.000)\end{array}$ & $\begin{array}{l}-0.597^{* * * *} \\
(0.000)\end{array}$ \\
\hline $\begin{array}{l}\text { High Emotional Stability, } \\
\text { High Conscientiousness } \\
\text { Helpless (Age 16) }\end{array}$ & $\begin{array}{l}-0.278^{* *} \\
(0.010)\end{array}$ & $\begin{array}{l}-0.367^{* * *} \\
(0.005)\end{array}$ & $\begin{array}{c}-0.242^{*} \\
(0.050)\end{array}$ & $\begin{array}{l}-0.550^{* *} \\
(0.000)\end{array}$ & $\begin{array}{l}-0.477^{* * * *} \\
(0.000)\end{array}$ & $\begin{array}{l}-0.614^{* * *} \\
(0.000)\end{array}$ & $\begin{array}{l}-0.563^{* * *} \\
(0.000)\end{array}$ \\
\hline $\begin{array}{l}\text { High Emotional Stability, } \\
\text { Low Conscientiousness } \\
\text { Help (Age 16) }\end{array}$ & $\begin{array}{l}-1.025^{* * * *} \\
(0.000)\end{array}$ & $\begin{array}{l}-0.527^{* * * *} \\
(0.003)\end{array}$ & $\begin{array}{l}-0.206 \\
(0.215)\end{array}$ & $\begin{array}{l}-0.536^{* *} \\
(0.002)\end{array}$ & $\begin{array}{c}{ }^{k}-0.251 \\
(0.128)\end{array}$ & $\begin{array}{l}-0.585^{* * *} \\
(0.002)\end{array}$ & $\begin{array}{c}-0.371^{* *} \\
(0.045)\end{array}$ \\
\hline $\begin{array}{l}\text { High Emotional Stability, } \\
\text { Low Conscientiousness } \\
\text { Helpless (Age 16) }\end{array}$ & $\begin{array}{l}-0.301^{* *} \\
(0.029)\end{array}$ & $\begin{array}{l}-0.315^{* *} \\
(0.042)\end{array}$ & $\begin{array}{l}-0.201 \\
(0.181)\end{array}$ & $\begin{array}{l}-0.461^{\text {** }} \\
(0.005)\end{array}$ & $\begin{array}{l}-0.375^{* *} \\
(0.019)\end{array}$ & $\begin{array}{l}-0.677^{* * *} \\
(0.000)\end{array}$ & $\begin{array}{l}-0.611^{* * *} \\
(0.000)\end{array}$ \\
\hline $\begin{array}{l}\text { Low Emotional Stability, } \\
\text { High Conscientiousness } \\
\text { Help (Age 16) }\end{array}$ & $\begin{array}{l}-0.814^{* * *} \\
(0.000)\end{array}$ & $\begin{array}{l}-0.545^{\text {**** }} \\
(0.000)\end{array}$ & $\begin{array}{l}-0.278 * * \\
(0.036)\end{array}$ & $\begin{array}{l}-0.645^{* *} \\
(0.000)\end{array}$ & $\begin{array}{l}{ }^{k}-0.434^{* * * *} \\
(0.002)\end{array}$ & $\begin{array}{l}-0.590^{* * *} \\
(0.000)\end{array}$ & $\begin{array}{l}-0.409^{* * *} \\
(0.010)\end{array}$ \\
\hline $\begin{array}{l}\text { Low Emotional Stability, } \\
\text { High Conscientiousness } \\
\text { Helpless (Age 16) }\end{array}$ & $\begin{array}{l}0.067 \\
(0.576)\end{array}$ & $\begin{array}{l}-0.110 \\
(0.423)\end{array}$ & $\begin{array}{l}-0.122 \\
(0.341)\end{array}$ & $\begin{array}{l}-0.223 \\
(0.115)\end{array}$ & $\begin{array}{c}-0.251^{*} \\
(0.063)\end{array}$ & $\begin{array}{c}-0.266^{*} \\
(0.072)\end{array}$ & $\begin{array}{l}-0.303^{* *} \\
(0.037)\end{array}$ \\
\hline $\begin{array}{l}\text { Low Emotional Stability, } \\
\text { Low Conscientiousness } \\
\text { Help (Age 16) }\end{array}$ & $\begin{array}{l}-0.763^{* * *} \\
(0.000)\end{array}$ & $\begin{array}{c}{ }^{k}-0.188 \\
(0.263)\end{array}$ & $\begin{array}{l}0.070 \\
(0.664)\end{array}$ & $\begin{array}{l}-0.506^{* *} \\
(0.001)\end{array}$ & $\begin{array}{l}-0.302^{* *} \\
(0.036)\end{array}$ & $\begin{array}{l}-0.602^{* * *} \\
(0.000)\end{array}$ & $\begin{array}{l}-0.456^{* * *} \\
(0.004)\end{array}$ \\
\hline $\begin{array}{l}\text { High Emotional Stability, } \\
\text { High Conscientiousness } \\
\text { Help Male (Age 16) }\end{array}$ & $\begin{array}{l}0.118 \\
(0.473)\end{array}$ & $\begin{array}{l}0.036 \\
(0.861)\end{array}$ & $\begin{array}{l}-0.029 \\
(0.878)\end{array}$ & $\begin{array}{l}0.162 \\
(0.407)\end{array}$ & $\begin{array}{l}0.146 \\
(0.438)\end{array}$ & $\begin{array}{l}0.139 \\
(0.499)\end{array}$ & $\begin{array}{l}0.160 \\
(0.420)\end{array}$ \\
\hline $\begin{array}{l}\text { High Emotional Stability, } \\
\text { High Conscientiousness } \\
\text { Helpless Male (Age 16) }\end{array}$ & $\begin{array}{l}0.057 \\
(0.741)\end{array}$ & $\begin{array}{l}-0.021 \\
(0.921)\end{array}$ & $\begin{array}{l}-0.094 \\
(0.627)\end{array}$ & $\begin{array}{l}0.268 \\
(0.189)\end{array}$ & $\begin{array}{l}0.254 \\
(0.198)\end{array}$ & $\begin{array}{l}0.263 \\
(0.220)\end{array}$ & $\begin{array}{l}0.277 \\
(0.182)\end{array}$ \\
\hline $\begin{array}{l}\text { High Emotional Stability, } \\
\text { Low Conscientiousness } \\
\text { Help Male (Age 16) }\end{array}$ & $\begin{array}{l}0.416^{* *} \\
(0.049)\end{array}$ & $\begin{array}{l}0.192 \\
(0.490)\end{array}$ & $\begin{array}{l}0.041 \\
(0.876)\end{array}$ & $\begin{array}{l}0.203 \\
(0.446)\end{array}$ & $\begin{array}{l}0.091 \\
(0.723)\end{array}$ & $\begin{array}{l}0.009 \\
(0.973)\end{array}$ & $\begin{array}{l}-0.042 \\
(0.870)\end{array}$ \\
\hline $\begin{array}{l}\text { High Emotional Stability, } \\
\text { Low Conscientiousness } \\
\text { Helpless Male (Age 16) }\end{array}$ & $\begin{array}{l}0.123 \\
(0.557)\end{array}$ & $\begin{array}{l}-0.099 \\
(0.685)\end{array}$ & $\begin{array}{l}-0.165 \\
(0.476)\end{array}$ & $\begin{array}{l}0.115 \\
(0.628)\end{array}$ & $\begin{array}{l}0.076 \\
(0.741)\end{array}$ & $\begin{array}{l}0.160 \\
(0.508)\end{array}$ & $\begin{array}{l}0.139 \\
(0.551)\end{array}$ \\
\hline $\begin{array}{l}\text { Low Emotional Stability, } \\
\text { High Conscientiousness } \\
\text { Help Male (Age 16) }\end{array}$ & $\begin{array}{l}0.064 \\
(0.750)\end{array}$ & $\begin{array}{l}0.191 \\
(0.429)\end{array}$ & $\begin{array}{l}0.176 \\
(0.444)\end{array}$ & $\begin{array}{l}0.110 \\
(0.652)\end{array}$ & $\begin{array}{l}0.120 \\
(0.612)\end{array}$ & $\begin{array}{l}0.225 \\
(0.397)\end{array}$ & $\begin{array}{l}0.249 \\
(0.336)\end{array}$ \\
\hline $\begin{array}{l}\text { Low Emotional Stability, } \\
\text { High Conscientiousness }\end{array}$ & $\begin{array}{l}-0.163 \\
(0.406)\end{array}$ & $\begin{array}{l}-0.503^{* *} \\
(0.023)\end{array}$ & $\begin{array}{l}-0.477^{* *} \\
(0.021)\end{array}$ & $\begin{array}{l}-0.357 \\
(0.103)\end{array}$ & $\begin{array}{l}-0.304 \\
(0.146)\end{array}$ & $\begin{array}{l}-0.306 \\
(0.165)\end{array}$ & $\begin{array}{l}-0.255 \\
(0.237)\end{array}$ \\
\hline
\end{tabular}


... Table 6.27 continued

\begin{tabular}{|c|c|c|c|c|c|c|c|}
\hline Mental Ill-Health Score ... & Age 16 & Age 26 & & All 34 & & All 42 & \\
\hline \multicolumn{8}{|l|}{ Helpless Male (Age 16) } \\
\hline $\begin{array}{l}\text { Low Emotional Stability, } \\
\text { Low Conscientiousness } \\
\text { Help Male (Age 16) }\end{array}$ & $\begin{array}{l}0.358^{*} \\
(0.085)\end{array}$ & $\begin{array}{l}-0.057 \\
(0.832)\end{array}$ & $\begin{array}{l}-0.145 \\
(0.579)\end{array}$ & $\begin{array}{l}0.122 \\
(0.603)\end{array}$ & $\begin{array}{l}0.019 \\
(0.935)\end{array}$ & $\begin{array}{l}0.067 \\
(0.782)\end{array}$ & $\begin{array}{l}0.073 \\
(0.759)\end{array}$ \\
\hline $\begin{array}{l}\text { Malaise Score } \\
\text { (Age 16) }\end{array}$ & & & $\begin{array}{l}0.108^{* * *} \\
(0.000)\end{array}$ & & $\begin{array}{l}0.098^{* * *} \\
(0.000)\end{array}$ & & $\begin{array}{l}0.080^{* * *} \\
(0.000)\end{array}$ \\
\hline Male & $\begin{array}{l}-0.394^{* * *} \\
(0.007)\end{array}$ & $\begin{array}{l}-0.381^{* *} \\
(0.032)\end{array}$ & $\begin{array}{l}-0.234 \\
(0.155)\end{array}$ & $\begin{array}{l}-0.357^{* *} \\
(0.043)\end{array}$ & $\begin{array}{l}-0.250 \\
(0.141)\end{array}$ & $\begin{array}{l}-0.369^{* *} \\
(0.044)\end{array}$ & $\begin{array}{c}-0.309^{*} \\
(0.080)\end{array}$ \\
\hline$N$ & 2309 & 1832 & 1832 & 1999 & 1999 & 1775 & 1775 \\
\hline$R^{2}$ & 0.197 & 0.137 & 0.205 & 0.104 & 0.162 & 0.120 & 0.158 \\
\hline
\end{tabular}

Source: British Cohort Study (BCS70), own calculations.

Note: $p$-values in parentheses $* p<0.10,{ }^{* *} p<0.05$, *** $p<0.01$. The OLS regressions include controls for mother's age, mother's age squared, region of living and parental socioeconomic background at birth, the personality traits agreeableness and extraversion measured at the age of 16 as well as cognitive ability measured at the age of 10 .

Table 6.28: Interaction between Hopelessness and Personality

\begin{tabular}{|c|c|c|c|c|c|c|}
\hline Mental Ill-Health Score ... & Age 16 & Age 26 & & All 34 & All 42 & \\
\hline $\begin{array}{l}\text { High Emotional Stability, } \\
\text { High Conscientiousness } \\
\text { Hope (Age 16) }\end{array}$ & $\begin{array}{l}-0.348^{* * *} \\
(0.003)\end{array}$ & $\begin{array}{l}-0.680 * * * \\
(0.000)\end{array}$ & $\begin{array}{l}-0.551^{* * * *} \\
(0.000)\end{array}$ & $\begin{array}{c}-0.742^{* * *}-0.644^{* * * *} \\
(0.000) \\
(0.000)\end{array}$ & $\begin{array}{l}-0.676^{* * * *} \\
(0.000)\end{array}$ & $\begin{array}{l}*-0.599 * * * \\
(0.000)\end{array}$ \\
\hline $\begin{array}{l}\text { High Emotional Stability, } \\
\text { High Conscientiousness } \\
\text { Hopeless (Age 16) }\end{array}$ & $\begin{array}{l}-0.273^{* *} \\
(0.033)\end{array}$ & $\begin{array}{l}-0.588^{* * *} \\
(0.000)\end{array}$ & $\begin{array}{l}-0.489^{* * * *} \\
(0.001)\end{array}$ & $\begin{array}{c}-0.735^{* * *}-0.671^{* * *} \\
(0.000) \\
(0.000)\end{array}$ & $\begin{array}{l}-0.541^{* * * *} \\
(0.001)\end{array}$ & $\begin{array}{l}*-0.497^{* * *} \\
(0.002)\end{array}$ \\
\hline $\begin{array}{l}\text { High Emotional Stability, } \\
\text { Low Conscientiousness } \\
\text { Hope (Age 16) }\end{array}$ & $\begin{array}{l}-0.447^{* * *} \\
(0.001)\end{array}$ & $\begin{array}{l}-0.678^{* * * *} \\
(0.000)\end{array}$ & $\begin{array}{l}-0.549^{* * * *} \\
(0.000)\end{array}$ & $\begin{array}{c}-0.659^{* * *}-0.534^{* * * *} \\
(0.000) \quad(0.000)\end{array}$ & $\begin{array}{l}-0.557^{* * * *} \\
(0.001)\end{array}$ & $\begin{array}{l}{ }^{*}-0.463^{* * *} \\
(0.005)\end{array}$ \\
\hline $\begin{array}{l}\text { High Emotional Stability, } \\
\text { Low Conscientiousness } \\
\text { Hopeless (Age 16) }\end{array}$ & $\begin{array}{l}-0.156 \\
(0.427)\end{array}$ & $\begin{array}{l}-0.297 \\
(0.201)\end{array}$ & $\begin{array}{l}-0.213 \\
(0.343)\end{array}$ & $\begin{array}{cc}-0.454^{* *} & -0.385^{*} \\
(0.046) & (0.084)\end{array}$ & $\begin{array}{l}-0.710^{* * * *} \\
(0.001)\end{array}$ & $\begin{array}{l}{ }^{*}-0.654^{* * *} \\
(0.002)\end{array}$ \\
\hline $\begin{array}{l}\text { Low Emotional Stability, } \\
\text { High Conscientiousness } \\
\text { Hope (Age 16) }\end{array}$ & $\begin{array}{l}-0.092 \\
(0.469)\end{array}$ & $\begin{array}{l}-0.527^{* * *} \\
(0.001)\end{array}$ & $\begin{array}{l}-0.487^{* * * *} \\
(0.001)\end{array}$ & $\begin{array}{c}{ }^{*}-0.511^{* * *}-0.494^{* * *} \\
(0.001) \\
(0.000)\end{array}$ & $\begin{array}{l}-0.316^{*} \\
(0.051)\end{array}$ & $\begin{array}{l}-0.301^{*} \\
(0.057)\end{array}$ \\
\hline $\begin{array}{l}\text { Low Emotional Stability, } \\
\text { High Conscientiousness } \\
\text { Hopeless (Age 16) }\end{array}$ & $\begin{array}{l}-0.010 \\
(0.943)\end{array}$ & $\begin{array}{l}-0.314^{*} \\
(0.064)\end{array}$ & $\begin{array}{l}-0.323^{* *} \\
(0.037)\end{array}$ & $\begin{array}{l}-0.487^{* * *}-0.485 * * * \\
(0.002) \quad(0.001)\end{array}$ & $\begin{array}{l}-0.422^{* *} \\
(0.011)\end{array}$ & $\begin{array}{l}-0.427^{* * *} \\
(0.008)\end{array}$ \\
\hline
\end{tabular}


... Table 6.28 continued

\begin{tabular}{|c|c|c|c|c|c|c|c|}
\hline Mental Ill-Health Score ... & Age 16 & Age 26 & & All 34 & & All 42 & \\
\hline $\begin{array}{l}\text { Low Emotional Stability, } \\
\text { Low Conscientiousness } \\
\text { Hope (Age 16) }\end{array}$ & $\begin{array}{l}-0.154 \\
(0.263)\end{array}$ & $\begin{array}{l}-0.423^{* * * *} \\
(0.009)\end{array}$ & $\begin{array}{l}-0.381^{* *} \\
(0.012)\end{array}$ & $\begin{array}{l}-0.570^{* * * *} \\
(0.000)\end{array}$ & $\begin{array}{l}-0.521^{* * * *} \\
(0.000)\end{array}$ & $\begin{array}{c}-0.381^{* *} \\
(0.026)\end{array}$ & $\begin{array}{l}-0.349 * * \\
(0.034)\end{array}$ \\
\hline $\begin{array}{l}\text { High Emotional Stability, } \\
\text { High Conscientiousness } \\
\text { Hope Male (Age 16) }\end{array}$ & $\begin{array}{l}0.105 \\
(0.590)\end{array}$ & $\begin{array}{l}0.459^{* *} \\
(0.034)\end{array}$ & $\begin{array}{l}0.379^{*} \\
(0.064)\end{array}$ & $\begin{array}{l}0.368^{*} \\
(0.099)\end{array}$ & $\begin{array}{l}0.330 \\
(0.122)\end{array}$ & $\begin{array}{l}0.136 \\
(0.588)\end{array}$ & $\begin{array}{l}0.124 \\
(0.605)\end{array}$ \\
\hline $\begin{array}{l}\text { High Emotional Stability, } \\
\text { High Conscientiousness } \\
\text { Hopeless Male (Age 16) }\end{array}$ & $\begin{array}{l}0.004 \\
(0.985)\end{array}$ & $\begin{array}{l}0.580^{* *} \\
(0.016)\end{array}$ & $\begin{array}{l}0.533^{* *} \\
(0.021)\end{array}$ & $\begin{array}{l}0.429^{*} \\
(0.076)\end{array}$ & $\begin{array}{l}0.432^{*} \\
(0.063)\end{array}$ & $\begin{array}{l}0.151 \\
(0.585)\end{array}$ & $\begin{array}{l}0.173 \\
(0.511)\end{array}$ \\
\hline $\begin{array}{l}\text { High Emotional Stability, } \\
\text { Low Conscientiousness } \\
\text { Hope Male (Age 16) }\end{array}$ & $\begin{array}{l}0.393^{*} \\
(0.075)\end{array}$ & $\begin{array}{l}0.658^{* * * *} \\
(0.007)\end{array}$ & $\begin{array}{l}0.521^{* *} \\
(0.026)\end{array}$ & $\begin{array}{l}0.419^{*} \\
(0.094)\end{array}$ & $\begin{array}{l}0.298 \\
(0.216)\end{array}$ & $\begin{array}{l}-0.036 \\
(0.894)\end{array}$ & $\begin{array}{l}-0.121 \\
(0.638)\end{array}$ \\
\hline $\begin{array}{l}\text { High Emotional Stability, } \\
\text { Low Conscientiousness } \\
\text { Hopeless Male (Age 16) }\end{array}$ & $\begin{array}{l}-0.137 \\
(0.632)\end{array}$ & $\begin{array}{l}0.131 \\
(0.703)\end{array}$ & $\begin{array}{l}0.107 \\
(0.749)\end{array}$ & $\begin{array}{l}0.092 \\
(0.778)\end{array}$ & $\begin{array}{l}0.097 \\
(0.756)\end{array}$ & $\begin{array}{l}0.296 \\
(0.415)\end{array}$ & $\begin{array}{l}0.338 \\
(0.327)\end{array}$ \\
\hline $\begin{array}{l}\text { Low Emotional Stability, } \\
\text { High Conscientiousness } \\
\text { Hope Male (Age 16) }\end{array}$ & $\begin{array}{l}-0.087 \\
(0.692)\end{array}$ & $\begin{array}{l}0.219 \\
(0.348)\end{array}$ & $\begin{array}{l}0.210 \\
(0.347)\end{array}$ & $\begin{array}{l}-0.119 \\
(0.610)\end{array}$ & $\begin{array}{l}-0.100 \\
(0.657)\end{array}$ & $\begin{array}{l}-0.400 \\
(0.128)\end{array}$ & $\begin{array}{l}-0.395 \\
(0.119)\end{array}$ \\
\hline $\begin{array}{l}\text { Low Emotional Stability, } \\
\text { High Conscientiousness } \\
\text { Hopeless Male (Age 16) }\end{array}$ & $\begin{array}{l}-0.001 \\
(0.997)\end{array}$ & $\begin{array}{l}0.294 \\
(0.266)\end{array}$ & $\begin{array}{l}0.297 \\
(0.249)\end{array}$ & $\begin{array}{l}0.213 \\
(0.423)\end{array}$ & $\begin{array}{l}0.197 \\
(0.444)\end{array}$ & $\begin{array}{l}0.205 \\
(0.483)\end{array}$ & $\begin{array}{l}0.217 \\
(0.445)\end{array}$ \\
\hline $\begin{array}{l}\text { Low Emotional Stability, } \\
\text { Low Conscientiousness } \\
\text { Hope Male (Age 16) }\end{array}$ & $\begin{array}{l}0.268 \\
(0.231)\end{array}$ & $\begin{array}{l}0.794^{* * * *} \\
(0.002)\end{array}$ & $\begin{array}{l}0.701^{* * *} \\
(0.004)\end{array}$ & $\begin{array}{l}0.439^{*} \\
(0.084)\end{array}$ & $\begin{array}{l}0.328 \\
(0.178)\end{array}$ & $\begin{array}{l}0.087 \\
(0.755)\end{array}$ & $\begin{array}{l}0.028 \\
(0.917)\end{array}$ \\
\hline $\begin{array}{l}\text { Malaise Score } \\
\text { (Age 16) }\end{array}$ & & & $\begin{array}{l}0.107^{* * * *} \\
(0.000)\end{array}$ & & $\begin{array}{l}0.101^{* * * *} \\
(0.000)\end{array}$ & & $\begin{array}{l}0.086 * * * \\
(0.000)\end{array}$ \\
\hline Male & $\begin{array}{l}-0.415^{* *} \\
(0.019)\end{array}$ & $\begin{array}{l}-0.865^{* * *} \\
(0.000)\end{array}$ & $\begin{array}{l}-0.706^{* * * *} \\
(0.000)\end{array}$ & $\begin{array}{l}-0.536^{\text {**** }} \\
(0.009)\end{array}$ & $\begin{array}{l}-0.402^{* *} \\
(0.040)\end{array}$ & $\begin{array}{l}-0.320 \\
(0.170)\end{array}$ & $\begin{array}{l}-0.227 \\
(0.306)\end{array}$ \\
\hline $\begin{array}{l}N \\
R^{2}\end{array}$ & $\begin{array}{l}2309 \\
0104\end{array}$ & $\begin{array}{l}1832 \\
0135\end{array}$ & $\begin{array}{l}1832 \\
0.210\end{array}$ & $\begin{array}{l}1999 \\
0096\end{array}$ & $\begin{array}{l}1999 \\
0166\end{array}$ & $\begin{array}{l}1775 \\
0107\end{array}$ & $\begin{array}{l}1775 \\
0157\end{array}$ \\
\hline
\end{tabular}

Source: British Cohort Study (BCS70), own calculations.

Note: $p$-values in parentheses $* p<0.10,{ }^{*} p<0.05,{ }^{* * *} p<0.01$. The OLS regressions include controls for mother's age, mother's age squared, region of living and parental socioeconomic background at birth, the personality traits agreeableness and extraversion measured at the age of 16 as well as cognitive ability measured at the age of 10 . 
Table 6.29: Helplessness Change, Gender and Mental Ill-Health

\begin{tabular}{|c|c|c|c|c|c|c|}
\hline Mental Ill-Health Score ... & Age 30 & & Age 34 & & All 42 & \\
\hline $\begin{array}{l}\text { Helplessness } \\
\text { (Age 16) }\end{array}$ & $\begin{array}{l}0.437 * * * \\
(0.000)\end{array}$ & $\begin{array}{l}0.331^{* * *} \\
(0.000)\end{array}$ & $\begin{array}{l}0.353^{* * *} \\
(0.000)\end{array}$ & $\begin{array}{l}0.249 * * * \\
(0.000)\end{array}$ & $\begin{array}{l}0.291^{* * *} \\
(0.000)\end{array}$ & $\begin{array}{l}0.203^{* * *} \\
(0.000)\end{array}$ \\
\hline $\begin{array}{l}\text { Helplessness Change } \\
(\text { Age } 30-16)>1\end{array}$ & $\begin{array}{l}0.619^{* * * *} \\
(0.000)\end{array}$ & $\begin{array}{l}0.576^{* * *} \\
(0.000)\end{array}$ & $\begin{array}{l}0.413^{* * *} \\
(0.000)\end{array}$ & $\begin{array}{l}0.372^{* * *} \\
(0.000)\end{array}$ & $\begin{array}{l}0.305^{* * *} \\
(0.000)\end{array}$ & $\begin{array}{l}0.272^{* * *} \\
(0.000)\end{array}$ \\
\hline $\begin{array}{l}\text { Helplessness Change } \\
\text { (Age } 30-16)<-1\end{array}$ & $\begin{array}{l}-0.565^{* * *} \\
(0.000)\end{array}$ & $\begin{array}{l}-0.539^{* * *} \\
(0.000)\end{array}$ & $\begin{array}{l}-0.394^{* * *} \\
(0.000)\end{array}$ & $\begin{array}{l}-0.370^{* * *} \\
(0.000)\end{array}$ & $\begin{array}{l}-0.341^{* * *} \\
(0.000)\end{array}$ & $\begin{array}{l}-0.303^{* * *} \\
(0.000)\end{array}$ \\
\hline $\begin{array}{l}\text { Helplessness Change } \\
\text { Male (Age } 30-16)\rangle 1\end{array}$ & $\begin{array}{l}0.052 \\
(0.590)\end{array}$ & $\begin{array}{l}0.020 \\
(0.836)\end{array}$ & $\begin{array}{l}-0.103 \\
(0.333)\end{array}$ & $\begin{array}{l}-0.128 \\
(0.220)\end{array}$ & $\begin{array}{l}0.020 \\
(0.860)\end{array}$ & $\begin{array}{l}0.006 \\
(0.956)\end{array}$ \\
\hline $\begin{array}{l}\text { Helplessness Change } \\
\text { Male (Age 30-16) }<-1\end{array}$ & $\begin{array}{l}-0.054 \\
(0.509)\end{array}$ & $\begin{array}{l}-0.020 \\
(0.800)\end{array}$ & $\begin{array}{l}-0.167^{*} \\
(0.086)\end{array}$ & $\begin{array}{l}-0.118 \\
(0.211)\end{array}$ & $\begin{array}{l}-0.077 \\
(0.461)\end{array}$ & $\begin{array}{l}-0.057 \\
(0.575)\end{array}$ \\
\hline $\begin{array}{l}\text { Emotional Stability } \\
\text { (Age 16) }\end{array}$ & $\begin{array}{l}-0.130 * * * \\
(0.000)\end{array}$ & $\begin{array}{l}-0.102^{* * *} \\
(0.000)\end{array}$ & $\begin{array}{l}-0.092^{* * *} \\
(0.001)\end{array}$ & $\begin{array}{l}-0.063^{* *} \\
(0.025)\end{array}$ & $\begin{array}{l}-0.180^{* * *} \\
(0.000)\end{array}$ & $\begin{array}{l}-0.158^{* * *} \\
(0.000)\end{array}$ \\
\hline $\begin{array}{l}\text { Conscientiousness } \\
\text { (Age 16) }\end{array}$ & $\begin{array}{l}-0.027 \\
(0.252)\end{array}$ & $\begin{array}{l}-0.025 \\
(0.279)\end{array}$ & $\begin{array}{l}-0.084^{* * *} \\
(0.003)\end{array}$ & $\begin{array}{l}-0.080^{* * *} \\
(0.003)\end{array}$ & $\begin{array}{l}-0.002 \\
(0.939)\end{array}$ & $\begin{array}{l}0.001 \\
(0.966)\end{array}$ \\
\hline $\begin{array}{l}\text { Malaise Score } \\
(\text { Age 16) }\end{array}$ & & $\begin{array}{l}0.082^{* * * *} \\
(0.000)\end{array}$ & & $\begin{array}{l}0.082^{* * *} \\
(0.000)\end{array}$ & & $\begin{array}{l}0.065^{* * *} \\
(0.000)\end{array}$ \\
\hline Male & $\begin{array}{l}-0.272^{* * *} \\
(0.000)\end{array}$ & $\begin{array}{l}-0.207^{* * *} \\
(0.000)\end{array}$ & $\begin{array}{l}-0.165^{* *} \\
(0.010)\end{array}$ & $\begin{array}{l}-0.102 \\
(0.111)\end{array}$ & $\begin{array}{l}-0.220^{* * *} \\
(0.001)\end{array}$ & $\begin{array}{l}-0.169^{* * *} \\
(0.009)\end{array}$ \\
\hline $\begin{array}{l}N \\
R^{2}\end{array}$ & $\begin{array}{l}2308 \\
0.231\end{array}$ & $\begin{array}{l}2308 \\
0.269\end{array}$ & $\begin{array}{l}1999 \\
0.161\end{array}$ & $\begin{array}{l}1999 \\
0.199\end{array}$ & $\begin{array}{l}1775 \\
0.149\end{array}$ & $\begin{array}{l}1775 \\
0.172\end{array}$ \\
\hline
\end{tabular}

Source: British Cohort Study (BCS70), own calculations.

Note: $p$-values in parentheses ${ }^{*} p<0.10,{ }^{* *} p<0.05,{ }^{* * *} p<0.01$. The OLS regressions include controls for mother's age, mother's age squared, region of living and parental socioeconomic background at birth, the personality traits agreeableness and extraversion measured at the age of 16 as well as cognitive ability measured at the age of 10 . 
Table 6.30: Helplessness Change, Personality and Mental Ill-Health

\begin{tabular}{lllllll}
\hline Mental Ill-Health Score $\ldots$ & Age 30 & & Age 34 & \multicolumn{3}{c}{ All 42 } \\
\hline Helplessness (Age 16) & $0.448^{* * *}$ & $0.328^{* * *}$ & $0.362^{* * *}$ & $0.246^{* * *}$ & $0.305^{* * *}$ & $0.199^{* * *}$ \\
& $(0.000)$ & $(0.000)$ & $(0.000)$ & $(0.000)$ & $(0.000)$ & $(0.000)$
\end{tabular}

High Emotional Stability, Low Conscientiousness (Age 16)

Low Emotional Stability, High Conscientiousness (Age 16)

Low Emotional Stability, Low Conscientiousness (Age 16)

High Emotional Stability, High Conscientiousness Helplessness Change (Age 30-16) > 1

High Emotional Stability, High Conscientiousness Helplessness Change (Age 30-16) $<-1$

High Emotional Stability, Low Conscientiousness Helplessness Change (Age 30-16) > 1

High Emotional Stability, Low Conscientiousness Helplessness Change (Age 30-16) $<-1$

Low Emotional Stability, High Conscientiousness Helplessness Change (Age 30-16) > 1

Low Emotional Stability, High Conscientiousness Helplessness Change (Age 30-16) $<-1$

$\begin{array}{llllll}0.001 & 0.011 & 0.092 & 0.102 & -0.018 & -0.029 \\ (0.989) & (0.889) & (0.344) & (0.281) & (0.859) & (0.770)\end{array}$

$\begin{array}{llllll}0.115 & 0.086 & 0.152^{*} & 0.119 & 0.255^{* * *} & 0.223^{* *} \\ (0.141) & (0.261) & (0.094) & (0.183) & (0.007) & (0.017)\end{array}$

$\begin{array}{llllll}0.197^{* *} & 0.165^{* *} & 0.311^{* * *} & 0.274^{* * *} & 0.363^{* * *} & 0.344^{* * *} \\ (0.019) & (0.042) & (0.002) & (0.006) & (0.001) & (0.001)\end{array}$

$\begin{array}{llllll}0.630^{* * *} & 0.579^{* * *} & 0.413^{* * *} & 0.365^{* * *} & 0.364^{* * *} & 0.325^{* * *} \\ (0.000) & (0.000) & (0.000) & (0.000) & (0.000) & (0.000)\end{array}$

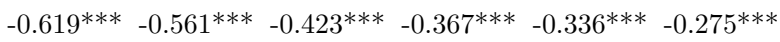
$\begin{array}{lllll}(0.000) & (0.000) & (0.000) & (0.000) & (0.000)\end{array}$

$\begin{array}{llllll}0.539^{* * *} & 0.494^{* * *} & 0.295^{*} & 0.262 & 0.233 & 0.228 \\ (0.000) & (0.000) & (0.082) & (0.111) & (0.155) & (0.157)\end{array}$

$\begin{array}{llllll}-0.500^{* * * *} & -0.461^{* * *} & -0.442^{* * *} & -0.400^{* * *} & -0.248 & -0.184 \\ (0.000) & (0.000) & (0.003) & (0.006) & (0.101) & (0.207)\end{array}$

$\begin{array}{llllll}0.518^{* * *} & 0.452^{* * *} & 0.192 & 0.128 & 0.238 & 0.195 \\ (0.000) & (0.000) & (0.167) & (0.352) & (0.109) & (0.185)\end{array}$

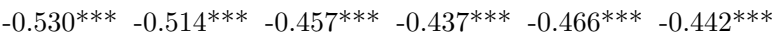

$\begin{array}{llllll}(0.000) & (0.000) & (0.000) & (0.000) & (0.000) & (0.000)\end{array}$ 
6 PESSIMISTIC EXPECTATIONS AND MENTAL HEALTH

... Table 6.30 continued

\begin{tabular}{lllllll}
\hline Mental Ill-Health Score ... & Age 30 & & Age 34 & \multicolumn{3}{c}{ All 42 } \\
\hline Low Emotional Stability, & $0.821^{* * *}$ & $0.723^{* * *}$ & $0.466^{* * *}$ & $0.380^{* * *}$ & $0.317^{* *}$ & 0.219 \\
Low Conscientiousness & $(0.000)$ & $(0.000)$ & $(0.001)$ & $(0.007)$ & $(0.038)$ & $(0.143)$
\end{tabular}

Helplessness Change

(Age 30-16) > 1

Low Emotional Stability,

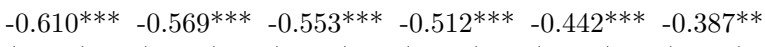

Low Conscientiousness

$\begin{array}{lllll}(0.000) & (0.000) & (0.000) & (0.000) & (0.005)\end{array}$

Helplessness Change

(Age 30-16) $<-1$

Malaise Score (Age 16)

$$
\begin{array}{lll}
0.090^{* * *} & 0.089^{* * *} & 0.075^{* * *} \\
(0.000) & (0.000) & (0.000)
\end{array}
$$

\begin{tabular}{lllllll}
\hline$N$ & 2308 & 2308 & 1999 & 1999 & 1775 & 1775 \\
$R^{2}$ & 0.209 & 0.257 & 0.144 & 0.191 & 0.127 & 0.159 \\
\hline
\end{tabular}

Source: British Cohort Study (BCS70), own calculations.

Note: $p$-values in parentheses ${ }^{*} p<0.10,{ }^{* *} p<0.05,{ }^{* * *} p<0.01$. The OLS regressions include controls for mother's age, mother's age squared, region of living and parental socioeconomic background at birth, the personality traits agreeableness and extraversion measured at the age of 16 as well as cognitive ability measured at the age of 10 . 
CHAPTER 7

DISCUSSION AND CONCLUSION 


\subsection{Main Results}

The personality trait neuroticism is observed to be negatively related to educational and occupational achievement, financial well-being, marriage or health outcomes, while the personality trait conscientiousness is mostly found to be a predictor for socioeconomic success throughout life. However, neuroticism seems to be not inevitably related to a socioeconomic disadvantage and some people concerned are more resilient than others. What drives such differences? To what extent is conscientiousness able to substitute for neuroticism in relation to socioeconomic outcomes by compensating for potentially hindering personality facets? And, what is the role of pessimistic expectations in the association between neuroticism and socioeconomic outcomes?

The dissertation aims to go beyond the current economic literature by addressing these questions from both a theoretical and an empirical perspective. First, a theoretical economic framework built on the human capital tradition and fed with insights from the psychological literature is developed (Chapter 2). Second, the hypothesized relationship between neuroticism and socioeconomic outcomes, the substitution effect of conscientiousness and the role of pessimistic expectations are empirically investigated using rich BCS70 cohort data (Chapters 3, 4, 5 and 6). The main findings are summarized as follows.

Chapter 2 provides insights from psychology and presents the theoretical economic framework. Studies in psychology argue that emotionally instable individuals have a higher stress-reactivity in challenging situations and are more likely to avoid problems rather than solving them due to pessimistic expectations. This is likely to translate into worse outcomes in various areas of life such as education achievement and might result in feelings of sustained stress and mental health problems. Conscientiousness might have the potential to break the vicious circle of negative expectations and experiences, because conscientious individuals are more likely to apply problem-focused strategies.

Putting these psychological theories into economic terms, the economic model argues that the individual aims to solve an influenceable, challenging situation with a utility-maximizing level of effort determined by the expected benefits and costs related to that effort. Individuals with lower levels of emotional stability are likely to have lower expected benefits owing to pessimistic beliefs. This is likely related to pro- 
crastination or even avoidance behavior, i.e. lower effort. However, procrastination or avoidance are likely to result in lower socioeconomic outcomes and contribute to the development of mental health problems, because the perceived and actual problem-solving ability is reduced and the uncomfortable challenge is likely to mentally and actually persist, i.e. lower utility. Higher conscientiousness might be able to mitigate the negative relation based on a higher probability of engagement and problem-focused coping which is likely to be associated with improved socioeconomic outcomes and empowerment.

Chapters 3 and 4 empirically analyze the economic framework by focusing on the objective measure educational achievement. Chapter 3 investigates the predictive power of (1) low emotional stability at the age of 16 for educational achievement at the age of 30 as well as the possible substitution effect of conscientiousness and (2) a change in emotional stability and conscientiousness between the ages of 10 and 16 for educational achievement at the age of 30. The analysis shows that women who score low in both emotional stability and conscientiousness at the age of 16 have the lowest educational achievement at the age of 30. Higher levels of conscientiousness at the age of 16 significantly mitigate the negative relation. An increase in emotional stability between the ages of 10 and 16 is significantly associated with improved educational achievement. For men, emotional stability seems to be less of concern, but conscientiousness is the crucial predictor for educational achievement. Both women and men who decrease in conscientiousness between the ages of 10 and 16 have significantly lower education outcomes at the age of 30. Particularly for women, the results are in line with the theoretical considerations. Taking into account that the regressions control for cognitive ability at the age of 10, the results are not only statistically significant, but also economically meaningful.

Chapter 4 focuses on the role of pessimistic expectations in a school setting measured as the individual perception about one's own ability in exams. It analyzes the relation (1) between the personality traits emotional stability and conscientiousness at the ages of 10 and 16 and pessimistic expectations at the age of 16 and (2) between emotional stability, conscientiousness and pessimistic expectations at the age of 16 and educational achievement at the age of 30. For women, the study reveals that particularly emotional stability and conscientiousness is negatively related to the expectation of being bad in exams which is in line with the theoretical considerations. For men, different mechanisms seems to play a role that are discussed in 
the next subsection. For both women and men, pessimistic expectations regarding one's own ability in exams at the age of 16 significantly predict poor educational achievement. Moreover, the findings suggest that individuals who score low in both emotional stability and conscientiousness at the age of 16 and who are found to reach the lowest education outcomes at the age of 30 have a significantly better education outcome in adulthood if they do not have pessimistic expectations in adolescence. This result is mainly in line with the theoretical considerations and suggests that programs in school aiming to adjust pessimistic expectations and promote the individual problem-solving ability might be able to boost education outcomes.

Chapters 5 and 6 focus on the subjective measure mental health problems. Chapter 5 analyzes the predictive power of (1) low emotional stability at the age of 16 for mental health problems at the ages of 16, 26, 30, 34 and 42 as well as the possible substitution effect of conscientiousness and (2) a change in emotional stability and conscientiousness between the ages of 10 and 16 and mental ill-health in later age. The study finds that emotional stability at the age of 16 significantly negatively predicts mental health problems in adolescence and adulthood. Individuals who score low in both emotional stability and conscientiousness at the age of 16 have the highest risk of experiencing mental health problems in adolescence and adulthood. Higher levels of conscientiousness at the age of 16 significantly mitigate the positive relation at adult age. Emotional stability at the age of 10 significantly predicts mental ill-health at the age of 16 and older. Individuals who increase in emotional stability between the ages of 10 and 16 are significantly less likely to experience mental health problems at the age of 16 and older, while a decrease in emotional stability is associated with an increased risk of mental ill-health. The results are again in line with the theoretical considerations and emphasize the importance of personality development in puberty for later life mental health outcomes.

Finally, Chapter 6 once more addresses pessimistic expectations that are now measured as thoughts of helplessness and hopelessness. It targets the relation (1) between the personality traits emotional stability and conscientiousness at the ages of 10 and 16 and pessimistic expectations at the age of $16,(2)$ between pessimistic expectations at the age of 16 and mental ill-health at later ages and (3) between a change in pessimistic expectations between the ages of 16 and 30 and mental health problems at later ages. The analysis reveals that low emotionally stable children and adolescents are more likely to have pessimistic expectations and that pessimistic 
expectations at the age of 16 are predictive of mental health outcomes in adolescence and adulthood. However, individuals who score low in both emotional stability and conscientiousness at the age of 16 and who are considered to have the highest risk for mental health problems are significantly less likely to experience mental ill-health in adolescence and adult age if they do not have pessimistic expectations in adolescence. Additionally, an increase in pessimistic expectations between the ages of 16 and 30 is significantly related to an increase in mental ill-health, while a decrease in pessimistic expectations is significantly associated with a decrease in mental health problems at the ages of 30,34 and 42. This suggests once more that school programs aiming to adjust pessimistic expectations and promote the individual problem-solving ability might be able to reduce mental health problems.

\subsection{Discussion of Results}

The economic model presented here adds to human capital theory by considering variation in individual effort and utility related to differences in personality traits and expectations. The theoretical considerations suggest that particularly low emotionally stable and low conscientious individuals who have pessimistic expectations are more likely to react with procrastination or avoidance in challenging situations (i.e. lower effort) based on short-run gains related to a reduction of negative emotions. However, these short-run benefits are likely to be outweighed by the long-run costs (i.e. lower utility). Nevertheless, both short-term benefits and the self-fulfilling prophecy related to destructive expectations and negative experiences makes it likely that particularly this group at-risk unconsciously and repetitively uses maladaptive and ineffective coping strategies related to poor long-term outcomes.

Both higher conscientiousness and more realistic expectations are assumed to slow down or even stop the vicious circle of self-fulfilling prophecies. Conscientiousness is regarded as a proxy for a more organized and self-disciplined mind-set as well as problem-focused coping behavior, which is likely to increase control and the probability of making positive experiences of success and mastering. Less negatively biased expectations related to more favorable personality traits or improved environmental conditions are expected to decrease arousal and effort invested in challenging situations.

The theoretical considerations are empirically tested by using the objective measure 
educational achievement and the subjective measure mental health in adulthood. If the hypothesized relationships exist, variation in both outcomes related to personality traits and pessimistic expectations should go hand in hand. The results are in line with the theory and suggest that particularly both low emotionally stable and low conscientious individuals who have pessimistic expectations are at-risk of having relatively poor education and mental health outcomes throughout life. Scoring higher in conscientiousness or having improved expectations significantly mitigates the negative association.

Roberts (2009) argues that thoughts and expectations are directly malleable by the environment. Adjusting pessimistic expectations - both on either personal development or more desirable environmental conditions - might help to boost educational achievement and reduce mental health problems by reducing sustained arousal and promoting problem-solving behavior in challenging situations.

The findings further suggest that conscientiousness is particularly important for educational achievement, while emotional stability more robustly predicts mental health outcomes. Referring back to the theoretical considerations, this might indicate that conscientiousness is particularly linked to the external actual problem-solving ability, i.e. finishing tasks and reaching own goals by the help of an appropriate work organization, time management, ambition and self-discipline. Emotional stability might be more related to the internal perceived problem-solving ability, i.e. having realistic expectations of being able to master a challenge successfully and experiencing less sustained arousal.

The results show significant gender differences. It is found that less emotionally stable women are more likely to have pessimistic expectations and are more vulnerable to pessimistic expectations compared to men which is in line with the psychological literature. Women are expected to be more likely to have pessimistic expectations compared to men, because they for instance experience on average more negative life events in childhood and have a higher appraisal of negative life events due to on average lower emotional stability and differences in gender socialization (Hankin and Abramson, 2001; Hyde et al., 2008). Moreover, women are more vulnerable to low emotional stability and pessimistic expectations compared to men, because women tend to ruminate more than men, which is considered as another type of cognitive vulnerability to stress (Hankin and Abramson, 2001; Hyde et al., 2008; Rood et al., 2012). This might make low emotionally stable women to be a particular group 
at-risk.

Finally, the study suggests that low emotionally stable, but more conscientious men who have pessimistic expectations have significantly better education and mental health outcomes compared to women with the same characteristics. The analysis shows that their education outcomes in adult age are even slightly better compared to emotionally stable and conscientious men who do not have pessimistic expectations in adolescents. For mental health outcomes, the results suggest that both groups of men narrow over time.

One might argue that these men are simply not affected by low emotional stability and pessimistic expectations. However, the analysis clearly reveals that pessimistic expectations predict poorer education and mental health outcomes. Therefore, it is likely that other mechanisms are at place. Arguing in terms of the model, it is likely that less emotionally stable, but more conscientious men who are pessimistic at the age of 16, i.e. theoretically considered as a group at-risk, act in contrast to the predictions of the model.

They might force themselves to overcome pessimistic expectations by putting effort instead of avoiding challenging situations. This is likely to result in positive experiences and better education outcomes, which is shown in the empirical analysis. Moreover, it is likely that pessimistic expectations become adjusted over time, which might boost control in challenging situations and is likely to reduce sustained stress. Accordingly, mental health problems are expected to become less prevalent in the long run, which is found in the regression results. These gender differences in coping strategies might be related to variation in societal expectations. Accordingly, Osborne (2000) studies personality differences and labor market outcomes and finds that men face a much higher wage penalty for withdrawal compared to women.

These gender differences in educational achievement and mental health outcomes indicate that the negative relation between low emotional stability or pessimistic expectations and socioeconomic outcomes is not set in stone, but a decrease in arousal and choosing the behavior alternative of problem-solving instead of problem avoidance might make a difference in terms of socioeconomic outcomes throughout life. Studies show that making positive experience in life can even change personality in the long-run (Scollon and Diener, 2006; van Aken et al., 2006; Lüdtke et al., 2011). Future research - preferably experimental studies - is needed to confirm the theoretical considerations and descriptive results and establish causal evidence. 


\subsection{Valorization Addendum}

The dissertation covers a topic that is highly relevant for both individuals and society yet has been understudied to date. Policies based on human capital theory mainly aim to reduce the impact of the family on education decisions and outcomes by eliminating credit constraints (Björklund and Kjell, 2011). Deficits in the personality traits emotional stability and conscientiousness related to pessimistic expectations, a higher stress-reactivity and a reduced problem-solving ability in unavoidable challenging situations as well as possible adverse consequences for mental health, education achievement and other important socioeconomic outcomes throughout life are still mostly neglected in many schools and assumed to be mainly the responsibility of the parents.

However, studies show that the child's level of emotional stability is both genetically and culturally highly influenced by the social environment and primarily the family. Thus, neuroticism is considered as $50 \%$ to $60 \%$ heritable, but can also develop based on continuous exposure to anxiety and stress (Cuijpers et al., 2010; Roberts, 2009). Similar findings also exists regarding the personality trait conscientiousness (Luciano et al., 2006; Eisenberg et al., 2014).

This makes it likely that the parents themselves either exhibit high neuroticism or low conscientiousness and are less likely to be able to support their children to compensate inherited and acquired personality deficits. Accordingly, the probable distress, problematic problem-solving strategies and the higher probability of experiencing failure in challenging situations in various life spheres is likely to remain untouched until it becomes obvious due to behavior and mental health problems or early school drop out.

Supporting particularly at-risk pupils by adjusting negative beliefs and expectations as well as fostering problem-solving skills might be a promising means to boost education outcomes and reduce mental health problems in adolescence and adult age. School interventions addressing the individual development, but also the environmental conditions might help to decrease mental and financial costs, improve life chances of particularly disadvantaged children and decrease intergenerational inequality. Cost-benefit studies based on existing school interventions suggest that the individual and social non-pecuniary and monetary benefits of such interventions throughout life are likely to exceed their costs. Therefore, policy-makers should in- 
creasingly become aware of these opportunities and use them to expand the common policy option of reducing credit constraints for education.

Psychological and economic studies provide insights into existing programs that aim to foster the individual development and support both parents or teachers in providing a more constructive and encouraging environment. In the following, some strategies that have the potential to break the destructive circle of self-fulfilling prophecies are exemplarily introduced.

Several studies show that the treatment of pessimistic beliefs and expectations is promising to boost mental health, but also other socioeconomic outcomes such as educational achievement. "Beck saw successful treatments as changes of the negative cognitive set (e.g., "I am an ineffective and helpless person") to a more positive set (Beck, 1974). He argued that the primary task [...] is to change the negative expectational scheme of the depressed patient to a more optimistic one. To reach this goal, it has to be explained to the patient where he is beginning to misinterpret reality. In a second step, the patient's thinking, i.e. his conceptions, can be modified by learning alternative and more positive conceptions" (Henkel et al., 2002, p. 246). ${ }^{19}$ This is in line with studies for nonclinical individuals. Reivich et al. (2013) review the Penn Resiliency Program (PRP) as an example of cognitive-behavioral school interventions and suggest that mental health problems such as depression and anxiety can be reduced by promoting accurate cognitive styles. Seligman (2006) provides techniques to challenge the individual negative explanatory style in negative life events, gives advice how to specifically enhance optimism in school and presents empirical evidence about the positive association between optimism and socioeconomic success in life.

Hopper and McHugh (2013) show that the college students' task performance in a learned helplessness situation is significantly better when using cognitive defusion techniques in comparison with a control group. Cognitive defusion is related to acceptance and commitment therapy (ACT, Hayes et al., 1999). "ACT clinicians encourage clients to defuse from their negative content by viewing their thoughts as just thoughts rather than considering them to have literal meaning (Healy et al., 2008)" (Hopper and McHugh, 2013, p. 210). Other related studies suggest that

19 This in line with several studies. See for instance Scott et al. (2000), Teasdale et al. (2000), Jarrett et al. (2001) and Paykel (2001). 
defusion techniques are efficient in dealing with unwanted pessimistic thoughts and they reduce the believability of negative statements as well as the associated level of distress (e.g., Masuda et al., 2004, 2009; Deacon et al., 2011).

Paunesku et al. (2015) conducted a programm intending to boost student's persistence in academic challenges. "The growth mindset intervention counteracts the fixed mindset [...], which is the belief that intelligence is a fixed entity that cannot be changed with experience and learning. The programm teaches scientific facts about the malleability of the brain, to show how intelligence can be developed. It then uses writing assignments to help students internalize the messages" (Claro et al., 2016, p. 375). They find that the intervention significantly boosts the grades among students at risk of high school drop-out. Claro et al. (2016) show that the growth mindset intervention is particularly beneficial for students from disadvantaged backgrounds.

Last but not least, Chaplin et al. (2006) designed a cognitive-behavioral and social problem-solving intervention to reduce and prevent depressive symptoms among girls. They find significantly positive effects that might arise due to the participant's social interaction, emerging friendships and exchange about destructive thoughts, problems and ways how to overcome them.

An overview and discussion about strategies targeting educational achievement or mental health outcomes in school is provided by Hosman et al. (2005), Gillham et al. (2006), Horowitz and Garber (2006), Young et al. (2006), Cardemil et al. (2007), Horowitz et al. (2007), Dignath and Büttner (2008), Hattie (2009), Stice et al. (2009), Weare and Nind (2011), Corrieri et al. (2014), Kautz et al. (2014), Carta et al. (2015) and Trier Damagaard and Skyt Nielsen (2017). The outcomes of these studies vary and depend on various factors such as type of intervention, target group, age of participants or duration and intensity of treatment. More research needs to be done to improve their effectiveness and efficiency.

Such interventions might not only be useful for individuals at risk, but also help to increase resilience in children and adolescence more generally. However, Horowitz and Garber (2006) and Stice et al. (2009) argue that larger effects result from programs that target high-risk individuals rather than universal programs. "Theoretically, the distress that characterizes high-risk individuals motivates these participants to engage more effectively in the prevention program [..." (Stice et al., 2009, p. 11). Moreover, also the duration and timing of programs is important. First, personality psychologists argue that long-run exposure at the state level is a requirement for a 
personality trait change (Roberts, 2009). Second, there is evidence that personality traits are malleable particularly in early age (Cunha et al., 2010). However, Stice et al. (2009) argue that successfully teaching the relevant concepts and skills requires a certain level of mental maturity. Therefore, adolescence seems to be the ideal time for successful intervention.

Finally, programs in school are particularly promising, because they guarantee high and longer run coverage as well as lower costs, because different public services can be combined. Carta et al. (2015) emphasize that "schools are a location where young people spend most of their daytime and socialize, schools are easily accessible to families, schools can provide non-stigmatizing health actions, and schools can provide appropriate and timely links with the community" (p. 17, see also World Health Organization, 2004).

Future research can build on (1) the economic framework and the descriptive analyses provided in the dissertation and (2) existing interventions to perform random control trails, test the theoretical framework causally and develop effective and efficient school programs. Thereby, it needs to be taken into account that transferring research-oriented studies into school practice is not without problems. Teachers might not understand all features of the intervention or differing school environments do not necessarily fit the study design (Borghans, Schils and de Wolf, 2016). Therefore, careful interdisciplinary guidance of the development, empirical analysis and implementation of school programs is essential. 



\section{BIBLIOGRAPHY}

Abramson, L. Y., Metalsky, G. I. and Alloy, L. B. (1989). Hopelessness Depression: A Theory-based Subtype of Depression, Psychological Review 96: 358-372.

Abramson, L. Y., Seligman, M. E. P. and Teasdale, J. (1978). Learned Helplessness in Humans: Critique and Reformulation, Journal of Abnormal Psychology 87: 4974.

Aldwin, C. M. and Revenson, T. A. (1987). Does Coping Help? A Reexamination of the Relation between Coping and Mental Health, Journal of Personality and Social Psychology 53(2): 337-348.

Allport, G. W. and Odbert, H. S. (1936). Traitnames. A Psycho-Lexical Study, Psychological Monographs 47: 1-171.

Almlund, M., Duckworth, A. L., Heckman, J. and Kautz, T. (2011). Personality Psychology and Economics, Vol. 4, Elsevier, Amsterdam, chapter 1, pp. 1-181.

Anderson, D. M., Cesur, R. and Tekin, E. (2015). Youth Depression and Future Criminal Behavior, Economic Inquiry 53(1): 294-317.

Bardasi, E. and Francesconi, M. (2004). The Effect of Non-Standard Employment on Mental Health in Britain, Social Science and Medicine 58(9): 1671-1688.

Baron, J. D. and Cobb-Clark, D. A. (2010). Are Young People's Educational Outcomes Linked to their Sense of Control?, IZA Discussion Paper 4907.

Beck, A. T. (1974). The Development of Depression: A Cognitive Model, in R. J. Friedman and M. M. Katz (eds), The Psychology of Depression: Contemporary Theory and Research, Winston \& Sons, Washington, pp. 3-20.

Becker, G. S. (1964). Human Capital. A Theoretical and Empirical Analysis, with Special Reference to Education, National Bureau of Economic Research, New York. 
Belsky, J., Hsieh, K. and Crnic, K. (1996). Infant Positive and Negative Emotionality: One Dimension or Two?, Developmental Psychology 32: 289-298.

Björklund, A. and Kjell, G. S. (2011). Education and Family Background: Mechanisms and Policies, in E. Hanushek, S. Machin and L. Woessmann (eds), Handbook of the Economics of Education, Vol. 3, North-Holland, Amsterdam, chapter 3, pp. 201-247.

Blázquez Cuesta, M. and Budría, S. (2015). Income Deprivation and Mental WellBeing: The Role of Non-Cognitive Skills, Economics \& Human Biology 17: 16-28.

Bogg, T. and Roberts, B. W. (2004). Concientiousness and Health-Related Behaviors: A Meta-Analysis of the Leading Behavioral Contributors to Mortality, Psychological Bulletin 130(6): 887-919.

Borghans, L., Duckworth, A. L., Heckman, J. J. and Ter Weel, B. (2008). The Economics and Psychology of Personality Traits, Journal of Human Resources 43(4): 972-1059.

Borghans, L., Golsteyn, B. H., Heckman, J. J. and Humphries, J. E. (2011). Identification Problems in Personality Psychology, Personality and Individual Differences 51(3): 315-320.

Borghans, L., Golsteyn, B. H., Heckman, J. J. and Humphries, J. E. (2016). What Grades and Achievement Tests Measure, Proceedings of the National Academy of Science of the United States of America 113(47): 13354-13359.

Borghans, L., Schils, T. and de Wolf, I. (2016). Experimentalism in Dutsch Education Policy, in T. Burns and F. Köster (eds), Governing Education in a Complex World, OECD Publishing, Paris, chapter 9, pp. 187-205.

Bratti, M. and Mendola, M. (2014). Parental Health and Child Schooling, Journal of Health Economics 35: 94-108.

Brodman, K., Erdmann, A., Lorge, I. and Broadbent, T. H. (1949). The Cornell Medical Index. An Adjunct to Medical Interview, Journal of the American Medical Association 140(6): 530-534.

Brodman, K., Erdmann, A., Lorge, I., Gershenson, C. P., Wolff, H. G. and Broadbent, T. H. (1952). The Cornell Medical Index. Health Questionnaire IV. The 
Recognition of Emotional Disturbances in a General Hospital, Journal of Clinical Psychology 8(3): 289-293.

Broidy, L. M., Tremblay, R. E., Brame, B., Fergusson, D., Horwood, J. L., Laird, R., Moffit, T. E., Nagin, D. S., Bates, J. E., Dodge, K. A., Loeber, R., Lynam, D. R. and Gregory, P. (2003). Developmental Trajectories of Childhood Disruptive Behaviors and Adolescent Delinquency: A Six-Site, Cross-National Study, Developmental Psychology 39(2): 222-245.

Bubonya, M., Cobb-Clark, D. A. and Wooden, M. (2017). Mental Health and Productivity at Work: Does What You Do Matter?, Labor Economics 46: 150-165.

Buis, M. L. (2010). Stata Tip 87: Interpretation of Interactions in Nonlinear Models, The Stata Journal 10(2): 305-308.

Butler, N., Bynner, J. and University of London, Institute of Education, Centre for Longitudinal Studies (2013). 1970 British Cohort Study: Sixteen-Year Follow-Up, 1986. 5th Edition. Colchester, Essex: UK Data Archive, May 2013. SN: 3535.

URL: http://dx.doi.org/10.5255/UKDA-SN-3535-2

Butler, N., Bynner, J. and University of London, Institute of Education, Centre for Longitudinal Studies (2014). 1970 British Cohort Study: Ten-Year Follow-Up, 1980. 5th Edition. Colchester, Essex: UK Data Archive, July 2014. SN: 3723.

URL: http://dx.doi.org/10.5255/UKDA-SN-3723-3

Bynner, J. and University of London, Institute of Education, Centre for Longitudinal Studies (2013). 1970 British Cohort Study: Twenty-Six-Year Follow-Up, 1996. 4th Edition. Colchester, Essex: UK Data Archive, May 2013. SN: 3833.

URL: http://dx.doi.org/10.5255/UKDA-SN-3833-2

Cahuc, P., Carcillo, S. and Zylberberg, A. (2014). Labor Economics, 2nd edn, MIT Press, Cambridge, MA.

Caliendo, M., Cobb-Clark, D. A., Seitz, H. and Uhlendorff, A. (2016). Locus of Control and Investment in Training, IZA Discussion Paper 10406.

Caliendo, M., Cobb-Clark, D. A. and Uhlendorff, A. (2015). Locus of Control and Job Search Strategies, Review of Economics and Statistics 97(1): 88-103. 


\section{BIBLIOGRAPHY}

Campbell-Sills, L., Cohan, S. L. and Stein, M. B. (2006). Relationship of Resilience to Personality, Coping, and Psychiatric Symptoms in Young Adults, Behaviour Research and Therapy 44(4): 585-599.

Cardemil, E. V., Reivich, K. J., Beevers, C. G., Seligman, M. E. and James, J. (2007). The Prevention of Depressive Symptoms in Low-income, Minority Children: Twoyear Follow-up, Behavior Research and Therapy 45(2): 313-327.

Carneiro, P. and Heckman, J. J. (2002). The Evidence on Credit Constraints in Post-Secondary Schooling, The Economic Journal 112: 705-734.

Carta, M. G., Di Fiandra, T., Rampazzo, L., Contu, P. and Preti, A. (2015). An Overview of International Literature on School Interventions to Promote Mental Health and Well-being in Children and Adolescents, Clinical Practice Es Epidemiology in Mental Health 11: 16-20.

Carver, C. S., Scheier, M. F. and Weintraub, J. K. (1989). Assessing Coping Strategies: A Theoretically Based Approach, Journal of Personality and Social Psychology 56(2): 267-283.

Cattell, R. B., Eber, H. W. and Tatsuoka, M. M. (1970). Handbook for the Sixteen Personality Factor Questionnaire, Institute for Personality and Ability Testing, Champaign.

Cervone, D. and Pervin, L. (2014). Personality Psychology, 12 edn, Wiley, New York.

Cesur, R., Sabia, J. J. and Tekin, E. (2013). The Psychological Costs of War: Military Combat and Mental Health, Journal of Health Economics 32(1): 51-65.

Chamberlain, R., Chamberlain, G. and University of London, I. (2013). Centre for Longitudinal Studies, 1970 British Cohort Study: Birth and 22-Month Subsample, 1970-1972. SN: 2666.

URL: http://dx.doi.org/10.5255/UKDA-SN-2666-2

Chaplin, T. M., Gillham, J. E., Reivich, K., Elkon, A. G. L., Samuels, B., Freres, D. R., Winder, B. and Seligman, M. E. P. (2006). Depression Prevention for Early Adolescent Girls: A Pilot Study of all Girls Versus Co-ed Groups, Journal of Early Adolescence 26(1): 110-126. 
Claro, S., Paunesku, D. and Dweck, C. S. (2016). Growth Mindset Tempers the Effects of Poverty on Academic Achievement, Proceedings of the National Academy of Sciences $\mathbf{2 0 1 6 0 8 2 0 7 .}$

Cobb-Clark, D. A., Kassenboehmer, S. C. and Schurer, S. (2014). Healthy Habits: The Connection between Diet, Exercise, and Locus of Control, Journal of Economic Behavior and Organization 98: 1-28.

Cobb-Clark, D. A. and Schurer, S. (2012). The Stability of Big-Five Personality Traits, Economics Letters 115(1): 11-15.

Connor-Smith, J. K. and Flachsbart, C. (2007). Relations between Personality and Coping: A Meta-Analysis, Journal of Personality and Social Psychology 93(6): 1080-1107.

Cornaglia, F., Crivellaro, E. and McNally, S. (2015). Mental Health and Education Decisions, Labour Economics 33: 1-12.

Cornaglia, F., Feldman, N. E. and Leigh, A. (2014). Crime and Mental Wellbeing, Journal of Human Resources 49(1): 110-140.

Corrieri, S., Heider, D., Conrad, I., Blume, A., König, H.-H. and Riedel-Heller, S. G. (2014). School-based Prevention Programs for Depression and Anxiety in Adolescence: a Systematic Review, Health Promotion International 29(3): 427441.

Costa, P. T. and McCrae, R. R. (1994). Set Like a Plaster? Evidence for the Stability of Adult Personality, in T. F. Heatherton and W. J. L. (eds), Can Personality Change?, American Psychological Association, Washington, DC, pp. 21-40.

Cottini, E. and Lucifora, C. (2013). Mental Health and Working Conditions in Europe, Industrial and Labor Relations Review 66(4): 958-988.

Cuijpers, P., Smit, F., Penninx, B. W., de Graaf, R., ten Have, M. and Beekman, A. T. (2010). Economic Costs of Neuroticism. A Population-Based Study, Archives of General Psychiatry 67(10): 1086-1093.

Cunha, F. and Heckman, J. J. (2007). The Technology of Skill Formation, American Economic Review $\mathbf{9 7}(2)$ : 31-47. 
Cunha, F., Heckman, J. J. and Schennach, S. (2010). Estimating the Technology of Cognitive and Noncognitive Skill Formation., Econometrica 78(3): 883-931.

Currie, J. and Moretti, E. (2003). Mother's Education and the Intergenerational Transmission of Human Capital: Evidence from College Openings and Longitudinal Data, Quarterly Journal of Economics 118(4): 1495-1532.

Danzer, A. M. and Danzer, N. (2016). The Long-Term Effects of the Chernobyl Catastrophe on Subjective Well-Being and Mental Health, Journal of Public Economics 135: 47-60.

De Paola, M. and Scoppa, V. (2015). Procrastination, Academic Success and the Effectiveness of Remedial Program, Journal of Economic Behavior and Organization 115: $217-236$.

Deacon, B. J., Fawzy, T. I., Lickel, J. J. and Wolitzky-Taylor, K. B. (2011). Cognitive Defusion Versus Cognitive Restructuring in the Treatment of Negative Selfreferential Thoughts: An Investigation of Process and Outcome, Journal of Cognitive Psychotherapy 25: 218-232.

Dignath, C. and Büttner, G. (2008). Components of Fostering Self-regulated Learning Among Students: A Meta-analysis on Intervention Studies at Primary and Secondary School Level, Metacognition Learing 3: 231-264.

Dodgeon, B., Hancock, M., Johnson, J. and Parsons, S. (2011). Deriving Highest Qualification in NCDS and BCS70, Data Note 2011/1, Centre for Longitudinal Studies, London.

Dohmen, T., Falk, A., Huffman, D. B. and Sunde, U. (2012). The Intergenerational Transmission of Risk and Trust Attitudes, Review of Economic Studies 79(2): 645677.

Drydakis, N. (2015). The Effect of Unemployment on Self-Reported Health and Mental Health in Greece from 2008 to 2013: A Longitudinal Study Before and During the Financial Crisis, Social Science and Medicine 128: 43-51.

Dunbar, J. P., McKee, L., Rakow, A., Watson, K. H., Forehand, R. and Compas, B. E. (2013). Coping, Negative Cognitive Style and Depressive Symptoms in Children of Depressed Parents, Cognitive Therapy and Research 37(1): 1-19. 
Dupuy, A. and Galichon, A. (2014). Personality Traits and the Marriage Market, Journal of Political Economy 122(6): 1271-1319.

Dustmann, C. and Fasani, F. (2016). The Effect of Local Area Crime on Mental Health, The Economic Journal 126(593): 978-1017.

Egan, M., Daly, M., Delaney, L., J., B. C. and Wood, A. M. (2017). Adolescent Conscientiousness Predicts Lower Lifetime Unemployment, Journal of Applied Psychology 102(4): 700-709.

Eisenberg, N., Duckworth, A. L., Spinrad, T. L. and Valiente, C. (2014). Conscientiousness: Origins in Childhood?, Developmental Psychology 50(5): 1331-1349.

Eurostat (2017). Population by Educational Attainment Level, Sex and Age (in Percent): edat_lfse_03 (accessed July 2017).

Eysenck (1992). Four Ways Five Factors are Basic, Personality and Individual Differences 13(6): 667-673.

Eyseneck, H. J. (1990). Biological Dimensions of Personality, Handbook of Personality: Theory and Research, Guilford Press, New York, pp. 244-276.

Falk, A., Becker, A., Dohmen, T., Enke, B., Huffman, D. B. and Sunde, U. N. . (2015). The Nature and Predictive Power of Preferences: Global Evidence, IZA Discussion Paper 9504.

Fehr, E., Glätzle-Rützler, D. and Sutter, M. (2013). The Development of Egalitarianism, Altruism, Spite and Parochialism in Childhood and Adolescence, European Economic Review 64: 369-383.

Fletcher, J. M. (2013). The Effects of Personality Traits on Adult Labor Market Outcomes: Evidence from Siblings, Journal of Economic Behavior $\mathcal{G}$ Organization 89: $122-135$.

Folkman, S. and Lazarus, R. S. (1985). If it Changes it Must be a Process: Study of Emotion and Coping During Three Stages of a College Examination, Journal of Personality and Social Psychology 48(1): 150-170.

Fouarge, D., Schils, T. and de Grip, A. (2013). Why Do Low-Educated Workers Invest Less in Further Training?, Applied Economics 45(18): 2587-2601. 


\section{BIBLIOGRAPHY}

Gabaix, X. and Laibson, D. (2016). Hyperbolic Discounting: Empirical Estimation, Policy Implications and Novel Interpretation. Presentation at Stirling Behavioral Science Centre Workshop on "Behavioral Science and Policy".

Gevrek, Z. E., Neumeier, C. and Gevrek, D. (2018). Explaining the Gender Test Score Gap in Mathematics: The Role of Gender Inequality, IZA Discussion Paper (11260).

Gillham, J. E., Reivich, K. J., Freres, D., Lascher, M., Litzinger, S., Shatte, A. and Seligman, M. (2006). School-based Prevention of Depression and Anxiety Symptoms in Early Adolescence: A Pilot of a Parent Intervention Component, School Psychology Quarterly 21(3): 323-348.

Goldberg, L. R. (1993). The Structure of Phenotypic Personality Traits, American Psychologist 48(1): 26-34.

Golsteyn, B. H. and Schils, T. (2014). Gender Gaps in Primary School Achievement: A Decomposition into Endowments and Returns to IQ and Non-cognitive Factors, Economics of Education Review 41: 176-187.

Grant, G., Nolan, M. and Ellis, N. (1990). A Reappraisal of the Malaise Inventory, Social Psychiatry and Psychiatric Epidemiology 25(4): 170-178.

Hankin, B. L. and Abramson, L. Y. (2001). Development of Gender Differences in Depression: An Elaborated Cognitive Vulnerability-Transactional Stress Theory, Psychological Bulletin 127: 773-796.

Hankin, B. L. and Abramson, L. Y. (2002). Measuring Cognitive Vulnerability to Depression in Adolescence: Reliability, Validity, and Gender Differences, Journal of Clinical Child and Adolescent Psychology 31(4): 491-504.

Hankin, B. L., Abramson, L. Y., Moffit, T. E., Silva, P. A., McGee, R. and Angell, K. E. (1998). Development of Depression from Preadolescents to Young Adultshood: Emerging Gender Differences in a 10-Year Longitudinal Study, Journal of Abnormal Psychology 107(1): 128-140.

Hattie, J. A. C. (2009). Visible Learning: A Synthesis of 800+ Meta-Analyses on Achievement, Routledge, Oxford. 
Hayes, S. C., Strohsahl, K. D. and Wilson, K. G. (1999). Acceptance and Commitment Theory: An Experimental Approach to Behavior Change, Guilford Press, New York, NY.

Healy, H. A., Barnes-Holmes, Y., Barnes-Holmes, D., Keogh, C., Luciano, C. and Wilson, K. (2008). An Experimental Test of Cognitive Defusion Exercise: Coping with Negative and Positive Self-Statements, The Psychological Record 58: 623640 .

Heckman, J. J., Lochner, L. J. and Todd, P. E. (2006). Earnings Functions, Rates of Return and Treatment Effects: The Mincer Equation and Beyond, in H. Eric and F. Welch (eds), Handbook of Econonomics of Education, Vol. 1, North-Holland, Amsterdam, chapter 7, pp. 307-458.

Heckman, J. J. and Rubinstein, Y. (2001). The Importance of Noncognitive Skills: Lessons from the GED Testing Program, The American Economic Association 91(2): 145-149.

Heckman, J. J., Stixrud, J. and Urzua, S. (2006). The Effects of Cognitive and Noncognitive Abilities on Labor Market Outcomes and Social Behavior, Journal of Labor Economics 24(3): 411-482.

Helliwell, J. F., Layard, R. and Sachs, R. (eds) (2013). World Happiness Report 2013, UN Sustainable Development Solutions Network, New York.

Henkel, V., Bussfeld, P., Möller, H.-J. and Hegerl, U. (2002). Cognitive-behavioural Theories of Helplessness/Hopelessness: Valid Models of Depression, European Archives of Psychiatry and Clinical Neuroscience 252: 240-249.

Hogan, R. and Hogan, J. (2007). Hogan Personality Inventory Manual, 3rd edn, Hogan Assessment Systems, Tulsa, OK.

Hopper, N. and McHugh, L. (2013). Cognitive Defusion Versus Thought Distraction in the Mitigation of Learned Helplessness, The Psychological Record 63: 209-218.

Horowitz, J. L. and Garber, J. (2006). The Prevention of Depressive Symptoms in Children and Adolescents: A Metaanalytic Review, Journal of Consulting and Clinical Psychology 74(3): 401-415. 


\section{BIBLIOGRAPHY}

Horowitz, J. L., Garber, J., Ciesla, J. A., Young, J. and Mufson, L. (2007). Prevention of Depressive Symptoms in Adolescents: A Randomized Trial of Cognitivebehavioral and Interpersonal Prevention Programs, Journal of Consulting and Clinical Psychology 75(5): 693-706.

Hosman, C., Jane-Llopis, E. and Saxena, S. (eds) (2005). Prevention of Mental Disorders: Effective Interventions and Policy Options, Oxford University Press, Oxford.

Hudson, E. and Barrett, A. (2014). Peer Groups, Employment Status and Depressive Symptoms among Older Adults in Ireland, Journal of Population Ageing 7(1): 4354.

Hyde, J. S., Mezulis, A. H. and Abramson, L. Y. (2008). The ABCs of Depression: Integrating Affective, Biological, and Cognitive Models to Explain the Emergence of the Gender Difference in Depression, Psychological Review 115(2): 291-313.

Jarrett, R. B., Kraft, D., Doyle, J., Foster, B. M., Eaves, G. G. and Silver, P. C. (2001). Preventing Recurrent Depression Using Cognitive Therapy With and Without a Continuation Phase: a Randomized Clinical Trial, Archives of General Psychiatry 58: 381-388.

Johnson, J. L. and Bloom, A. M. (1995). An Analysis of the Contribution of the Five Factors of Personality to Variance in Academic Performance, Personality and Individual Differences 18(1): 127-133.

Johnston, D. W., Schurer, S. and Shields, M. A. (2013). Exploring the Intergenerational Persistence of Mental Health: Evidence From Three Generations, Journal of Health Economics 32(6): 1077-1089.

Judge, T. A. and Bono, J. E. (2001). Relationship of Core Self-Evaluations TraitsSelf-esteem, Generalized Self-efficacy, Locus of Control, and Emotional Stabilitywith Job Satisfaction and Job Performance: A Meta-Analysis., Journal of Applied Psychology 86(1): 80-92.

Judge, T. A., Erez, A., Bono, J. E. and Thoresen, C. J. (2002). Are Measures of Selfesteem, Neuroticism, Locus of Control, and Generalized Self-Efficacy Indicators of a Common Core Construct?, Journal of Personality and Social Psychology 83(3): 693-710. 
Karasek, R. A. (1979). Job Demands, Job Decision Latitude, and Mental Strain: Implications for Job Redesign, Administrative Science Quarterly 24(2): 285-308.

Karasek, R. and Theorell, T. (1992). Healthy Work: Stress Productivity And The Reconstruction Of Working Life, Basis Book, United States.

Kautz, T., Heckman, J. J., Diris, R., Ter Weel, B. and Borghans, L. (2014). Fostering and Measuring Skills: Improving Cognitive and Non-cognitive Skills to Promote Lifetime Success, OECD Education Working Papers 110.

Kendler, K. and Myers, J. (2010). The Genetic and Environmental Relationship between Major Depression and the Five-factor Model of Personality, Psychological Medicine 40(5): 801-806.

Laibson, D. (1997). Golden Eggs and Hyperbolic Discounting, Quarterly Journal of Economics 112(2): 443-477.

Layard, R., Chisholm, D., Patel, V. and Saxena, S. (2013). Mental Illness and Unhappiness, IZA Discussion Paper $\mathbf{7 6 2 0 .}$

Layard, R., Clark, A. E., Cornaglia, F., Powdthavee, N. and Vernoit, J. (2014). What Predicts a Successful Life? A Life-Course Model of Well-Being, Economic Journal 124(580): F720- F738.

Lebel, C. and Beaulieu, C. (2011). Longitudinal Development of Human Brain Wiring Continues from Childhood into Adulthood, The Journal of Neuroscience 31(30): 10937-10947.

Lester, D. (2001). An Inventory to Measure Helplessness, Hopelessness, and Hapelessness, Psychological Reports 89: 495-498.

Lindeboom, M., Portrait, F. and van den Berg, G. J. (2002). An Econometric Analysis of the Mental-Health Effects of Major Events in the Life of Elderly Individuals, Health Economics 11(6): 505-520.

Lochner, L. (2011). Nonproduction Benefits of Education: Crime, Health, and Good Citizenship, in Hanushek Eric A., Machin Stephan and L. Woessmann (eds), Handbook of the Economics of Education, Vol. 4, Elsevier North-Holland, Amsterdam, chapter 2, pp. 183-282. 


\section{BIBLIOGRAPHY}

Lochner, L. and Monge-Naranjo, A. (2012). Credit Constraints in Education, Annual Review of Economics 4(1): 225-256.

Luciano, M., Wainwright, M. A., Wright, M. J. and Martin, N. G. (2006). The Heritability of Conscientiousness Facets and Their Relationship to IQ and Academic Achievement, Personality and Individual Differences 40: 1189-1199.

Lüdtke, O., Roberts, B. W., Trautwein, U. and Nagy, G. (2011). A Random Walk Down University Avenue: Life Path, Life Events, and Personality Trait Change at the Transition to University Life, Journal of Personality and Social Psychology 101(3): 620-637.

Lundberg, S. (2013). The College Type: Personality and Educational Inequality, Journal of Labor Economics 31(3): 421-441.

Machin, S., Marie, O. and Vujić, S. (2011). The Crime Reducing Effect of Education, Economic Journal 121: 463-484.

Machin, S., Pelkonen, P. and Salvanes, K. (2011). Education and Mobility, Journal of the European Economic Association 10(2): 417-450.

Masuda, A., Hayes, S. C., Sackett, C. F. and Twohig, M. P. (2004). Cognitive Defusion and Self-relevant Negative Thoughts: Examining the Impact of a Ninety Year Old Technique, Behavior Research and Therapy 42: 477-485.

Masuda, A., Hayes, S. C., Twohig, M. P., Drossel, C., Lillis, J. and Washio, Y. (2009). A Parametric Study of Cognitive Defusion and the Believability and Discomfort of Negative Self-relevant Thoughts, Behavior Modification 33: 250-262.

Matthews, G., Deary, I. J. and Whiteman, M. C. (2009). Personality Traits, Cambridge University Press, Cambridge.

McCrae, R. R. and John, O. P. (1992). An Introduction to the Five-Factor Model and its Applications, Journal of Personality 60(2): 175-215.

McEwen, B. S., Liston, C. and Morrison, J. H. (2006). Stress-induced Structural Plasticity in Prefrontal Cortex, Amygdala and Hippocampus, Neuropsychopharmacology 31: S 13.

McGee, R., Williams, S. and Silva, P. A. (1986). An Evaluation of the Malaise Inventory, Journal of Psychosomatic Research 30(2): 147-152. 
Mendolia, S. and Walker, I. (2014a). The Effect of Non-Cognitive Traits on Health Behaviours in Adolescence, Health Economics 23(9): 1146-1158.

Mendolia, S. and Walker, I. (2014b). The Effect of Personality Traits on Subject Choice and Performance in High School: Evidence from an English Cohort, Economics of Education Review 43: 47-65.

Mezulis, A., Hyde, J. S. and Abramson, L. Y. (2006). The Developmental Origins of Cognitive Vulnerability to Depression: Temperament, Parenting, and Negative Life Events in Childhood as Contributors to Negative Cognitive Style, Developmental Psychology 42(6): 1012-1025.

Nezlek, J. B. (2007). A Multilevel Framework for Understanding Relationships Among Traits, States, Situations and Behaviors, European Journal of Personality. 2007 21(6): 789-810.

OECD (2011). Sick on the Job? Myths and Reality about Mental Health and Work, OECD Publishing, Paris.

OECD (2016). Education at a Glance 2016: OECD Indicators, OECD Publishing, Paris.

Oreopoulos, P. and Salvanes, K. (2011). Priceless: The Nonpecuniary Benefits of Schooling, Journal of Economic Perspectives 25(1): 159-184.

Osborne, M. (2000). The Power of Personality: Labor Market Rewards and the Transmission of Earnings, University of Massachusetts.

Overmier, J. B. and Seligman, M. E. P. (1967). Effects on Inescapable Shock Upon Subsequent Escape and Avoidance Responding, Journal of Comparative and Physiological Psychology 63: 28-33.

Paunesku, D., Walton, G. M., Romero, C., Smith, E. N., Yeager, D. S. and Dweck, C. S. (2015). Mind-set Intervention are a Scalable Treatment for Academic Underachievement, Psychological Science 26(6): 784-793.

Paykel, E. S. (2001). Cognitive Therapy in Prevention of Relapse for Antidepressant-treated Patients with Residual Depression, World Journal of Biological Psychiatry 2: 169S. 
Penley, J. A., Tomaka, J. and Wiebe, J. S. (2002). The Association of Coping To Physical and Psychological Health Outcomes: A Meta-Analytic Review, Journal of Behavioral Medicine 25(6): 551-603.

Peracchi, F. (2006). Educational Wage Premia and the Distribution of Earnings: An International Perspective, in E. Hanushek and F. Welch (eds), Handbook of the Economics of Education, Vol. 1, North-Holland, chapter 5, pp. 189-254.

Persson, P. and Rossin-Slater, M. (2016). Family Ruptures, Stress, and the Mental Health of the Next Generation, American Economic Review forthcoming.

Polachek, S., Das, T. and Thamma-Apiroam, R. (2015). Micro and Macro Implications of Heterogeneity in the Production of Human Capital, Journal of Political Economy 123(6): 1410-1455.

Prevoo, T. and ter Weel, B. (2015). The Importance of Early Conscientiousness for Socio-Economic Outcomes: Evidence from the British Cohort Study, Oxford Economic Papers 67(4): 918-948.

Reivich, K., Gillham, J. E., Chaplin, T. M. and Seligman, M. E. P. (2013). From Helplessness to Optimism: The Role of Resilience in Treating and Preventing Drepression in Youth, Handbook of Resilience in Children, Springer, New York, NY, pp. 201-214.

Roberts, B. W. (1997). Plaster or Plasticity: Are Adult Work Experiences Associated with Personality Changes in Women?, Journal of Personality 65: 205-232.

Roberts, B. W. (2009). Back to the Future: Personality and Assessment and Personality Development, Journal of Research in Personality 43(2): 137-145.

Roberts, B. W. and Del Vecchio, W. F. (2000). The Rank-Order Consistency of Personality Traits from Childhood to Old Age: A Quantitative Review of Longitudinal Studies, Psychological Bulletin 126(1): 3-25.

Roberts, B. W., Kuncel, N. R., Shiner, R., Caspi, A. and Goldberg, L. R. (2007). The Power of Personality. The Comparative Validity of Personality Traits, Socioeconomic Status, and Cognitive Ability for Predicting Important Life Outcomes, Perspectives on Psychological Science 2(4): 313-345. 
Roberts, B. W., Walton, K. E. and Viechtbauer, W. (2006). Patterns of MeanLevel Change in Personality Traits Across the Life Course: A Meta-Analysis of Longitudinal Studies, Psychological Bulletin 132(1): 1-25.

Robins, Richard W., F. R. C., Roberts, B. W. and Trzesniewski, K. H. (2001). Plaster or Plasticity: Are Adult Work Experiences Associated with Personality Changes in Women?, Journal of Personality 69(4): 617-640.

Rodgers, B., Pickle, A., Power, C., Collishaw, S. and Maughan, B. (1999). Validity of the Malaise Inventory in General Population Samples, Social Psychiatry and Psychiatric Epidemiology 34(6): 333-341.

Rood, L., Roelofs, J. and Bögels, S. M. (2012). Stress-Reactive Rumination, Negative Cognitive Style, and Stressors in Relationship to Depressive Symptoms in NonClinical Youth, Journal of Youth Adolescence 41: 414-425.

Ruhm, C. J. (2012). Understanding Overeating and Obesity, Journal of Health Economics 31(6): 781-796.

Rustichini, A., DeYoung, C. G., Anderson, J. E. and Burks, S. V. (2016). Toward the Integration of Personality Theory and Decision Theory in the Explanation of Economic Behavior, Journal of Behavioral and Experimental Economics 64: 122137.

Rutter, M. and Madge, N. (1976). Cycles of Disadvantage, Heinemann, London.

Rutter, M., Tizard, J. and K., W. (1970). Education, Health and Behaviour, Longmans, London.

Ryan, M., Delaney, L. and Harmon, C. P. (2013). The Role of Noncognitive Traits in Undergraduate Study Behaviours, Economics of Education Review 32: 181-195.

Schmitt, D. P., Realo, A., Voracek, M. and Allik, J. (2008). Why Can't a Man Be More Like a Woman? Sex Differences in Big Five Personality Traits across 55 Cultures, Journal of Personality and Social Psychology 94(1): 168-182.

Scollon, C. N. and Diener, E. (2006). Love, Work, and Changes in Extraversion and Neuroticism Over Time, Journal of Personality and Social Psychology 91: 11521165. 
Scott, J., Teasdale, J. D., Paykel, E. S., Johnson, A. L., Abbott, R., Hayhurst, H., R., M. and Garland, A. (2000). Effects of Cognitive Therapy on Psychological Symptoms and Social Functioning in Residual Depression, British Journal of Psychiatry 177: 440-446.

Seligman, M. E. P. (1975). Helplessness, Freeman, San Francisco.

Seligman, M. E. P. (2006). Learned Optimism: How to Change Your Mind and Your Life, 3rd edn, Vintage, New York.

Siegrist, J. (1996). Adverse Health Effects of High-Effort-Low-Reward Conditions, Journal of Occupational Health Psychology 1(1): 27-41.

Steyer, R., Schmitt, M. and Eid, M. (1999). Latent State-Trait Theory and Research in Personality and Individual Differences, European Journal of Personality 13(5): 389-408.

Stice, E., Shaw, H., Bohon, C., Marti, C. N. and Rohde, P. (2009). A Meta-Analytic Review of Depression Prevention Programs for Children and Adolescents: Factors that Predict Magnitude of Intervention Effects, Journal of Consulting and Clinical Psychology 77(3): 486-503.

Straathof, B. and Weehuizen, R. (2005). Technological Change, Job Stress and Burnout, in L. Soete and B. Ter Weel (eds), The Economics of the Digital Society, Edward Elgar, Cheltenham, chapter 8, pp. 184-199.

Szirmai, A. (2005). The Dynamics of Socio-Economic Development. An Introduction., Cambridge University Press, Cambridge.

Teasdale, J. D., Segal, Z. V., Williams, J. M. G., Ridgeway, V. A., Soulsby, J. M. and Lau, M. A. (2000). Prevention of Relapse/Recurrence in Major Depression by Mindfulness-Based Cognitive Therapy, Journal of Consulting and Clinical Psychology 68(4): 615-623.

Tefft, N. (2012). Mental Health and Employment: The Sad Story, Economics 85 Human Biology 10(3): 242-255.

Trautwein, U., Lüdtke, O., Nagy, N., Lenski, A., Niggli, A. and Schnyder, I. (2015). Using Individual Interest and Conscientiousness to Predict Academic Effort: Additive, Synergistic, or Compensatory Effects?, Journal of Personality and Social Psychology 109(1): 142-162. 
Trier Damagaard, M. and Skyt Nielsen, H. (2017). Nudging in Education: A Survey, Aarhus University: Economic Working Papers 5: 1-35.

UK Data Service (2016). Background to the Malaise Inventory: Measure Levels of Psychological Distress or Depression.

URL: https://www.ukdataservice.ac.uk/teaching-resources/malaise/background

University of London. Institute of Education. Centre for Longitudinal Studies (2012). 1970 British Cohort Study: Forty-Two-Year Follow-Up, 2012. Colchester, Essex: UK Data Archive, March 2014. SN: 7473.

URL: http://dx.doi.org/10.5255/UKDA-SN-7473-1

University of London, Institute of Education, Centre for Longitudinal Studies (2013a). 1970 British Cohort Study: Thirty-Four-Year Follow-Up, 2004-2005. 3rd Edition. Colchester, Essex: UK Data Archive, May 2013. SN: 5585.

URL: http://dx.doi.org/10.5255/UKDA-SN-5585-2

University of London, Institute of Education, Centre for Longitudinal Studies (2013b). 1970 British Cohort Study: Twenty-Nine-Year Follow-Up, 1999-2000. 3rd Edition. Colchester, Essex: UK Data Archive, May 2013. SN: 5558.

URL: http://dx.doi.org/10.5255/UKDA-SN-5558-2

Ursin, H. and Eriksen, H. R. (2004). The Cognitive Activation Theory of Stress, Psychoneuroendocrinology 29(5): 567-92.

van Aken, M. A. G., Denissen, J. J. A., Branje, S. J. T., Dubas, J. S. and Goossens, L. (2006). Midlife Concers and Short-Term Personality Change in Middle Adulthood, European Journal of Personality 20: 497-513.

Vatan, S. and Lester, D. (2008). The Internal Consistency and Concurrent Validity of the Hopelessness, Helplessness, and Hapelessness Scale in a Clinical Sample, Psychological Reports 103: 701-702.

Weare, K. and Nind, M. (2011). Mental Health Promotion and Problem Prevention in Schools: What Does the Evidence Say?, Health Promotion International 26(S1): i29-i69.

Wilkinson, N. and Klaes, M. (2012). An Introduction to Behavioral Economics, 2 edn, Palgrave MacMillan, Basingstoke. 


\section{BIBLIOGRAPHY}

Williams, R. (2006). Generalized Ordered Logit: Partial Proportional Odds Models for Ordinal Dependent Variables, The Stata Journal 6(1): 58-82.

World Health Organization (2004). Summary Report: Prevention of Mental Disorders - Effective Interventions and Policy Options, WHO, Geneva.

Xu, Y., Beller, A. H., Roberts, B. W. and Brown, J. R. (2015). Personality and Young Adult Financial Distress, Journal of Economic Psychology 51: 90-100.

Young, J. F., Mufson, L. and Davies, M. (2006). Efficacy of Interpersonal Psychotherapy-Adolescent Skills Training: An Indicated Preventive Intervention for Depression, Journal of Child Psychology and Psychiatry 47(12): 1254-1262. 


\section{CURRICULUM VITAE}

Caroline Wehner was born in Plauen, Germany on June 1, 1979. She graduated from high school at the Freiherr-vom-Stein Gymnasium in Rösrath, Germany in 1999.

After high school, she investigated her interests and talents by doing an apprenticeship as IT-Merchant followed up by a position as IT-Trainer (1999-2003), by being self-employed as web developer (2003-2006), by studying Regional Science of LatinAmerica at Cologne University (2003-2005) or by working as a German Language Assistant in primary and secondary schools in France (2005-2006).

From 2006 until 2011, Caroline studied economics at Cologne and Utrecht University, specializing in empirical research and public policy. Beside her studies, she was working as student research assistant at the Leibniz Institute for the Social Sciences (GESIS), the Chair for Empirical Research in Social Science and Economics at Cologne University and the Institute for Labor Economics (IZA).

In 2011, she became a resident research affiliate at IZA in Bonn, Germany, where she was working until 2017. She started her PhD in economics at Maastricht and United Nations University in 2012. Her research has been presented at various international workshops and conferences.

Since April 2017, Caroline is a research associate at the Federal Institute for Vocational Education and Training (BIBB) in Bonn, Germany. 



\section{UNU-MERIT/MGSoG Dissertation Series}

2018

Stefania Innocenti

On Institutional Persistence

UNU-MERIT/MGSoG Dissertation

Series № 199

2017

Hans-Erik Edsand

Winds of Change

UNU-MERIT/MGSoG Dissertation

Series № 197

Ana Patricia Silva Vara

Redressing the Gender Gap

UNU-MERIT/MGSoG Dissertation

Series № 196

Andrés Iván Mideros Mora

Essays on the Economic Effects of Noncontributory Social Protection

UNU-MERIT/MGSoG Dissertation

Series № 195

Tobias Broich

New Actors in the Global Economy

UNU-MERIT/MGSoG Dissertation

Series № 194

Bernard Nikaj

From No-government to E-government UNU-MERIT/MGSoG Dissertation

Series № 193
Hassen Abda Wako

Economic Globalization, Institutions

and Development

UNU-MERIT/MGSoG Dissertation

Series № 198

Ali Safarnejad

Prioritizing the HIV Response

UNU-MERIT/MGSoG Dissertation

Series № 192

Clovis Freire

Diversification and Structural

Economic Dynamics

UNU-MERIT/MGSoG Dissertation

Series № 191

Michael Verba

Innovation and Knowledge Dynamics:

Essays on the Knowledge Economy

UNU-MERIT/MGSoG Dissertation

Series № 190

Pui Hang Wong

The Hearts and Minds in Conflict and

Peace: The Economics of

Counterinsurgency and the Psychology of Reconstruction

UNU-MERIT/MGSoG Dissertation

Series № 189 
Brenda Yamba

Schooling Despite All Odds: Evidence from Lesotho on Female Child Carers who Stayed in School

UNU-MERIT/MGSoG Dissertation

Series № 188

Sheng Zhong

Moving towards An Energy Efficient Future; Essays on Energy Efficiency,

Technology and Development

UNU-MERIT/MGSoG Dissertation

Series № 187
Julieta Marotta

Access to Justice and Legal

Empowerment of Victims of Domestic

Violence through Legal Organizations in the City of Buenos Aires; A

Qualitative Empirical Legal Study

UNU-MERIT/MGSoG Dissertation

Series, № 186

Andrea Franco-Correa

On the Measurement of

Multidimensional Poverty as a Policy

Tool; Empirical Applications to Chile,

Colombia, Ecuador and Peru

UNU-MERIT/MGSoG Dissertation

Series, № 185

Thuy Dieu Nguyen

Firm-Level Theory and Evidence of

Corruption

UNU-MERIT Dissertation Series, № 105

Raquel Tsukada Lehman

Essays on Household Production with

Labor-Saving Technology

UNU-MERIT Dissertation Series,

№ 104

Eva Barteková

Multi-Problem Challenges for a

Renewable Future; Empirical Studies

on Competitive Disadvantages from

Electricity Price Differentials and

Mineral Supply Risk in an Open

Economy

UNU-MERIT Dissertation Series,

№ 103 
Jocelyn Olivari

Entrepreneurial Traits and Innovation;

Evidence from Chile

UNU-MERIT Dissertation Series, № 102

Muhammad Shafique

Essays on the role of knowledge, RED, and Technology-based Firms in the Evolution of Socio-techno-economic System

UNU-MERIT Dissertation Series, № 101

Serdar Türkeli

Governance of Innovation Policy;

Empirical Studies on Applied Political Economy by Multi-Methods Analysis UNU-MERIT Dissertation Series, № 100

Ayokunu Adedokun

Pathways to Sustainable Peacebuilding in Divided Societies; Lessons and

Experiences from Mozambique

MGSoG Dissertation Series, № 75

Luiz Rothier Bautzer

Organizing Concurrent Engineering through ICT Platforms

Blueprinting Product Lifecycle

Management Platforms across

Disciplinary Agencies

MGSoG Dissertation Series, № 74
Natalia Popova

Migration in the Periphery of the

European Union;

Determinants of Successful and

Sustainable Labour Market Integration of Return Migrants in Albania, Egypt, Moldova and Tunisia

MGSoG Dissertations Series, № 73

Richard A. Martina

Uncertainty and Resource Constraint in the Small Island Developing States; Essays in Entrepreneurial Cognition MGSoG Dissertations Series, № 72

Cécile Cherrier

The Expansion of Basic Social Protection in Low-income Countries; An Analysis of Foreign Aid Actors'

Role in the Emergence of Social Transfers in Sub-Saharan Africa MGSoG Dissertations series, № 71

Paul Caldron

The Tacit Bargain in Short-Term Medical Missions; Why U.S. physicians go and what it costs MGSoG Dissertation Series, № 70

\section{Mahmut Kobal}

Customs \& Excellence: A Comparative Approach on Administrative and Regulatory Compliance Perspectives of the EU-Turkey Customs Union MGSoG Dissertation Series, № 69

Craig Loschmann

Essays on Conflict-related Migration and Development in the Case of Afghanistan

MGSoG Dissertations Series, № 68 
Andrea Milan

Rural Livelihoods, Location and

Vulnerable Environments; Approaches to Migration in Mountain areas of

Latin America

MGSoG Dissertation Series, № 67

2015

Hibret Belete Maemir

Dissecting Aggregate Productivity;

International Integration and Growth with Heterogeneous Firms

UNU-MERIT Dissertation Series, № 96

Giorgio Triulzi

Looking for the Right Path; Technology

Dynamics, Inventive Strategies and

Catching-up in the Semiconductor

Industry

UNU-MERIT Dissertation Series,

№ 95

Abdul Baseer Qazi

Knowledge flows and networks in the

ICT sector; The case of Pakistan

UNU-MERIT Dissertation Series,

№ 94

Ajay Thutupalli

Technology Paradigm Shifts in

Agriculture; Drivers of Sustainability

and Catch up

UNU-MERIT Dissertation Series,

№ 93
Farida Lada

On Guarding the Welfare of Clinical

Trial Subjects While Promoting Novel

Drug Innovation

A Game Theoretical Approach

MGSoG Dissertation Series, № 66

Eduardo Urias

Improving access to HIVIAIDS

treatment in Brazil; When are

Compulsory Licenses effective in Price

Negotiations?

UNU-MERIT Dissertation Series, № 92

Francesca Guadagno

Why have so few Countries

Industrialised?

UNU-MERIT Dissertation Series, № 91

Daniel Opolot

The Evolution of Beliefs and Strategic

Behaviour

UNU-MERIT Dissertation Series, № 90

Alejandro Lavopa

Structural Transformation and

Economic Development; Can

Development Traps be Avoided

UNU-MERIT Dissertation Series, № 89

Jinjin Zhao

Urban water management reform; The

Case of China

UNU-MERIT Dissertation Series, № 88 
Simona Vezzoli

Borders, Independence and Post-

colonial Ties; the Role of the State in

Caribbean Migration

MGSoG Dissertation Series, № 65

Silvia Consuelo Gómez Soler

Civil Conflict and Education; How

Does Exposure to Civil Conflict Affect

Human Capital Accumulation?

Evidence from Standardized Exit

Exams in Colombia

MGSoG Dissertation Series, № 64

Paula Nagler

Occupational Choice in the Developing

World

MGSoG Dissertation Series, № 63

Jasmin Kientzel

Determinants of Professional

Commitment to Environmental

Sustainability

MGSoG Dissertation Series, № 62

Mehmet Güney Celbiş

Regional Policies; Convergence, Trade, and the Allocation of Public Capital

MGSoG Dissertation Series, № 61

Florian Henning

Living Up to Standard;

Interoperability Governance and

Standards Adoption in Government

Information Networks

MGSoG Dissertation Series, № 60
Niels P. Groen

The Never-Ending Project

Understanding E-Government Project

Escalation

MGSoG Dissertation Series, № 59

Derek Copp

Teacher-Based Reactivity to Provincial

Large-scale Assessment in Canada

MGSoG Dissertation Series, № 58

Michaella Vanore

Family-Member Migration and the

Psychosocial Health Outcomes of

Children in Moldova and Georgia

MGSoG Dissertation Series, № 57

Sonja Fransen

The Economic and Social Effects of

Remittances and Return Migration in

Conflict-Affected Areas; The Case of

Burundi

MGSoG Dissertation Series, № 56

Ibrahim Khalil Conteh

The Impact of Floods on Primary

School Education in Zambia

MGSoG Dissertation Series, № 55

Richard Bluhm

Growth Dynamics and Development

Essays in Applied Econometrics and

Political Economy

MGSoG Dissertation Series, № 54

Nevena P. Zhelyazkova

Work-Family Reconciliation and Use of

Parental Leave in Luxembourg;

Empirical Analysis of Administrative

Records

MGSoG Dissertation Series, № 53 
Dirk Crass

The Impact of Brands on Innovation and Firm Performance; Empirical

Evidence from Germany UNU-MERIT Dissertation Series, № 87

Samyukta Bhupatiraju

The Geographic Dimensions of Growth and Development

UNU-MERIT Dissertation Series, № 86

François Lafond

The Evolution of Knowledge Systems

UNU-MERIT Dissertation Series, № 85

Annalisa Primi

Promoting Innovation in Latin

America; What Countries Have

Learned (and What They Have Not) in

Designing and Implementing

Innovation and Intellectual Property

Policies

UNU-MERIT Dissertation Series, № 84

Fatoumata Lamarana Diallo

Evaluation of Meal and Deworming

Programs for Primary Schools in Rural

Senegal

UNU-MERIT Dissertation Series, № 83
Sachin Kumar Badkas

Metachoice and Metadata; Innovating with Environmental Policy Analysis in Europe

MGSoG Dissertation Series, № 52

Irina S. Burlacu

An Evaluation of Tax-Benefit Systems

Impact on the Welfare of Frontier

Worker;

The Case of Luxembourg and Belgium MGSoG Dissertation Series, № 51

Özge Bilgili

Simultaneity in Transnational

Migration Research; Links Between

Migrants' Host and Home Country

Orientation

MGSoG Dissertation Series, № 50

Yulia Privalova Krieger

Reshaping the Big Agenda;

Transnational Politics and Domestic

Resistance Financial crisis and social

protection reform in Bosnia and

Herzegovina

MGSoG Dissertation Series, № 49

Marieke van Houte

Moving Back or Moving Forward?

Return migration after Conflict

MGSoG Dissertation Series, № 48

Oxana Slobozhan

Global Governance in the Management of Natural Resources; The Case of the

Extractive Industries Transparency

Initiative (EITI)

MGSoG Dissertation Series, № 47 
Luis Bernardo Mejia Guinand

The Changing Role of the Central

Planning Offices in Latin America; A

Comparative Historical Analysis

Perspective (1950-2013)

MGSoG Dissertation Series, № 46

Cheng Boon Ong

Ethnic Segregation in Housing,

Schools and Neighbourhoods in the

Netherlands

MGSoG Dissertation Series, № 45

Luciana V. Cingolani

Bureaucracies for Development;

Oxymoron or Reality? Studies on State

Capacity in Challenging Governance

Contexts

MGSoG Dissertation Series, № 44

Carlos Cadena Gaitán

Green Politics in Latin American

Cities - Sustainable Transport Agendas

MGSoG Dissertation Series, № 43
Katie Kuschminder

Female Return Migration and

Reintegration Strategies in Ethiopia

MGSoG Dissertation Series, № 42

Metka Hercog

Highly-Skilled Migration and New

Destination Countries

MGSoG Dissertation Series, № 41

Margaret Agaba Rugadya

Can Remittances Influence the Tenure and Quality of Housing in Uganda? MGSoG Dissertation Series, № 40

Ilire Agimi

New Governance Under Limited

Statehood; The Case of Local

Government Reform in Kosovo

MGSoG Dissertation Series, № 39

\section{3}

Anant Kamath

Information Sharing through Informal

Interaction in Low-Tech Clusters

UNU-MERIT Dissertation Series,

№ 82

Flavia Pereira de Carvalho

What we talk about when we talk about

Brazilian Multinationals; An

Investigation on Brazilian FDI,

Economic Structure, Innovation and

the Relationship between them

UNU-MERIT Dissertation Series, № 81
Jun Hou

Complementarity in Innovation and

Development; A Cross-country

Comparison

UNU-MERIT Dissertation Series, № 80

Rufin Baghana

Impacts of Government Incentives to RED, Innovation and Productivity;

A Micro econometric Analysis of the Québec Case

UNU-MERIT Dissertation Series, № 79 
Lilia I. Stubrin

High-Tech Activities in Emerging

Countries; A Network perspective on

the Argentinean Biotech Activity

UNU-MERIT/MGSoG Dissertation

Series, № 78

Kristine Farla

Empirical Studies on Institutions,

Policies and Economic Development

MGSoG Dissertation Series, № 38

Marina Petrovic

Social Assistance and Activation in the

Pursuit of Happiness; Shedding New

Light on Old Policy Solutions to Social

Exclusion

MGSoG Dissertation Series, № 37

Laura Torvinen

Assessing Governance Assessments;

The Case of Mozambique; Governance

Assessments in the Context of Aid

Effectiveness Discourse

MGSoG Dissertation Series, № 36
Biniam Egu Bedasso

Institutional Change in the Long

Shadow of Elite; Essays on

Institutions, Human Capital and

Ethnicity in Developing Countries

MGSoG Dissertation Series, № 35

Sepideh Yousefzadeh Faal Deghati

Childhoods Embargoed; Constructing

and Reconstructing Multidimensional

Child Poverty in Iran 1984-2009

MGSoG Dissertation Series, № 34

Robert Bauchmüller

Investing in Early Childhood Care and

Education; The Impact of Quality on

Inequality

MGSoG Dissertation Series, № 33

Martin Rehm

Unified Yet Separated; Empirical

Study on the Impact of Hierarchical

Positions within Communities of

Learning

MGSoG Dissertation Series, № 32

2012

Abdul Waheed

Innovation Determinants and

Innovation as a Determinant; Evidence

from Developing Countries

UNU-MERIT Dissertation Series,

№ 77

Bilal Mirza

Energy Poverty and Rural Energy

Markets in Pakistan

UNU-MERIT Dissertation Series,
Benjamin Engelstätter

Enterprise Software and Video Games;

An Empirical Analysis

UNU-MERIT Dissertation Series,

№ 75

Fulvia Farinelli

Natural Resources, Innovation and

Export Growth; The Wine Industry in

Chili and Argentina

UNU-MERIT Dissertation Series № 76 
Rodolfo Lauterbach

Innovation in Manufacturing; From

Product Variety and Labor

Productivity Growth to Economic

Development in Chile

UNU-MERIT Dissertation Series

Kirsten Wiebe

Quantitative Assessment of

Sustainable Development and Growth in Sub-Saharan Africa

UNU-MERIT/MGSoG Dissertation

Series, № 74

Julio Miguel Rosa

Organizational Strategies, Firms'

Performance and Spatial Spillovers;

The Canadian Case in Research and

Development.

UNU-MERIT Dissertation Series, № 73

Johannes Wilhelmus Marie Boels Joseph Schumpeter, Honderd Jaar

Economische Ontwikkeling; Een

Historisch-theoretische Beschouwing.

UNU-MERIT Dissertation Series
Dorcas Mbuvi

Utility Reforms and Performance of the Urban Water Sector in Africa

MGSoG Dissertation Series, № 31

Lina Salanauskaite

Distributional Impacts of Public

Policies; Essays in Ex-Ante and Ex-

Post Evaluation

MGSoG Dissertation Series, № 30

Esther Schüring

To Condition or not - is that the

Question?

An Analysis of the Effectiveness of ExAnte and Ex-Post Conditionality in

Social Cash Transfer Programs

MGSoG Dissertation Series, № 29

Joe Abah

Strong Organisations in Weak States; Atypical Public Sector Performance in

Dysfunctional Environments

MGSoG Dissertation Series, № 28

Zina Samih Nimeh

Social Citizenship Rights; Inequality

and Exclusion

MGSoG Dissertation Series, № 27

2011

Daniel Vertesy

Interrupted Innovation; Emerging

Economies in the Structure of the

Global Aerospace Industry

UNU-MERIT Dissertation Series, № 72
Tina Saebi

Successfully Managing Alliance

Portfolios; An Alliance Capability

View

UNU-MERIT Dissertation Series, № 71 
Nora Engel

Tuberculosis in India; A Case of

Innovation and Control

UNU-MERIT/MGSoG Dissertation

Series, № 70

Evans Mupela

Connectivity and growth in Sub-

Saharan Africa; The Role of

Communication Satellites

UNU-MERIT Dissertation Series,

№ 69

Nantawan Kwanjai

Cross Cultural Intelligence amid

Intricate Cultural Webs; A Tale of the

UnDutchables in the Land of 1002

Smiles

UNU-MERIT Dissertation Series, № 68

Lina Sonne

Innovation in Finance to Finance Innovation; Supporting Pro-poor

Entrepreneur-based Innovation

UNU-MERIT Dissertation Series, № 67

Lenka Eisenhamerová

Legitimacy of 'Humanitarian Military

Intervention'

MGSoG Dissertation Series, № 26

Sonila Tomini

Informal Payments for Health Care

Services in Albania

MGSoG Dissertation Series, № 25
Jinjing Li

Dynamic Microsimulation in Public

Policy Evaluation

MGSoG Dissertation Series, № 24

Aziz Atamanov

Rural Nonfarm Employment and

International Migration as

Alternatives to Agricultural

Employment; The Case of Kyrgyzstan

MGSoG Dissertation Series, № 23

Frieda Vandeninden

Poverty Alleviation; Aid and Social

Pensions

MGSoG Dissertation Series, № 22

Juliana Nyasha Tirivayi

The Welfare Effects of Integrating

AIDS Treatment with Food Transfers;

Evidence from Zambia

MGSoG Dissertation Series, № 21

Agnieska Ewa Sowa

Who's Left Behind? Social Dimensions

of Health Transition and Utilization of

Medical Care in Poland

MGSoG Dissertation Series, № 20

Emmanaouil Sfakianakis

The Role of Private Actors in the

Provision of Public Goods with

Applications to Infrastructure and

Financial Stability

MGSoG Dissertation Series, № 19 
Siu Hing Lo

White Collars Green Sleeves; An Interorganizational Comparison of Determinants of Energy-Related Behaviors among Office Workers MGSoG Dissertation Series, № 18
Treena $\mathrm{Wu}$

Constraints to Human Capital

Investment in Developing Countries; Using the Asian Financial Crisis in Indonesia as a Natural Experiment MGSoG Dissertation Series, № 17

Henry Espinoza Peña

Impact Evaluation of a Job-Training

Programme for Disadvantaged Youths;

The Case of Projoven

MGSoG Dissertation Series, № 16

2010

Fernando Santiago

Human Resources Management

Practices and Learning for Innovation in Developing Countries;

Pharmaceutical Firms in Mexico

UNU-MERIT Dissertation Series,

№ 66

Zakaria Babutsidze

Essays on Economies with

Heterogeneous Interacting Consumers

UNU-MERIT Dissertation Series, № 65

Bertha Vallejo

Learning and Innovation Under

Changing Market Conditions; The

Auto Parts Industry in Mexico

UNU-MERIT Dissertation Series, № 64
Donatus Ayitey

Technical Change, Competitiveness

and Poverty Reduction; A Study of the

Ghanaian Apparel Industry

UNU-MERIT Dissertation Series,

№ 63

\section{Sergey Filippov}

Multinational Subsidiary Evolution;

Corporate Change in New EU Member

States

UNU-MERIT Dissertation Series, № 62

\section{Asel Doranova}

Technology Transfer and Learning

under the Kyoto Regime; Exploring the Technological Impact of CDM Projects in Developing Countries

UNU-MERIT Dissertation Series, № 61 
Florian Tomini

Between Family and Friend;

Understanding the Interdependency of

Private Transfers

MGSoG Dissertation Series, № 15

Michał Polalowski

The Institutional Transformation of

Social Policy in East Central Europe;

Poland and Hungary in Comparative

and Historical Perspective

MGSoG Dissertation Series, № 14

Maha Ahmed

Defining, Measuring and Addressing

Vulnerability; The Case of Post

Conflict Environments

MGSoG Dissertation Series, № 13

Pascal Beckers

Local Space and Economic Success; The

Role of Spatial Segregation of Migrants in the Netherlands

MGSoG Dissertation Series, № 12

Victor Cebotari

Conflicting Demands in Ethnically

Diverse Societies; Ethno political

Contention and Identity Values in

Europe

MGSoG Dissertation Series, № 11
Dennis Gyllensporre

Competing and Complementary

Perspectives on the EU as a Crisis

Management Actor;

An Examination of the Common

Security and Defence Policy through

the Lenses of Idealism and Realism

MGSoG Dissertation Series, № 10

Judit Vall Castello

Business Cycle and Policy Effects on

Labour Market Transitions of Older

and Disabled Workers in Spain

MGSoG Dissertation Series, № 9

Keetie Roelen

False Positives or Hidden Dimensions;

The Definition and Measurement of

Child Poverty

MGSoG Dissertation Series, № 8

Denisa Maria Sologon

Earning Dynamics in Europe

MGSoG Dissertation Series, № 7

Melissa Siegel

Money and Mobility; Migration and

Remittances

MGSoG Dissertation Series, № 6

Jessica S. Hagen-Zanker

Modest Expectations; Causes and

Effects of Migration on Migrant

Households in Source Countries

MGSoG Dissertation Series, № 5 
Alexis Habiyaremye

From Primary Commodity Dependence to Diversification and Growth;

Absorptive Capacity and Technological Catch Up in Botswana and Mauritius.

UNU-MERIT Dissertation Series, № 60

Yoseph Getachew

The Role of Public Capital in Economic Development

UNU-MERIT Dissertation Series, № 59

Sandra Leitner

Embodied Technological Change and

Patterns of Investment in Austrian

Manufacturing

UNU-MERIT Dissertation Series, № 58

Semih Akçomak

The Impact of Social Capital on

Economic and Social Outcomes

UNU-MERIT Dissertation Series, № 57

\section{8}

Rutger Daems

Medicines for the Developing World UNU-MERIT Dissertation Series, № 54

\author{
Abraham Garcia \\ The Role of Demand in Technical \\ Change \\ UNU-MERIT Dissertation Series, \\ № 56
}

Saurabh Arora

Coherence in Socio-technical Systems;

A Network Perspective on the

Innovation Process

UNU-MERIT Dissertation Series, № 55

Mirtha R. Muniz Castillo

Human Development and Autonomy in Project Aid; Experiences from four bilateral projects in Nicaragua and $\mathrm{El}$ Salvador

MGSoG Dissertation Series, № 4

Christiane Arndt

Governance Indicators

MGSoG Dissertation Series, № 3

Britta Augsburg

Microfinance; Greater Good or Lesser

Evil?

MGSoG Dissertation Series, №

Johannes Hanel

Assessing Induced Technology;

Sombart's Understanding of Technical

Change in the History of Economics

UNU-MERIT Dissertation Series, № 53 
Rifka Weehuizen

Mental Capital; the Economic

Significance of Mental Health

UNU-MERIT Dissertation Series, № 52

Danielle Cloodt

The Relationship between RED

Partnership Formation, Social

Embeddedness and Innovative

Performance

UNU-MERIT Dissertation Series,

№ 51

2007

Tobias Kronenberg

Reconciling Environmental

Conservation with Economic

Prosperity; The Feasibility of Double

Dividends in the Short and Long Run

UNU-MERIT Dissertation Series,

№ 49

Viktoria Kravtsova

Assessing the Impact of Foreign Direct

Investment in Transition Economies

UNU-MERIT Dissertation Series,

№ 48

2006

Bulat Sanditov

Essays on Social Learning and

Imitation

UNU-MERIT Dissertation Series, № 46
Sabine Fuss

Sustainable Energy Development

under Uncertainty

UNU-MERIT Dissertation Series, № 50

Geranda Notten

Measuring and Managing Poverty

Risks

MGSoG Dissertation Series, № 1
Suhail Sultan

The Competitive Advantage of Small and Medium Sized Enterprises; The Case of Jordan's Natural Stone Industry

UNU-MERIT Dissertation Series, № 47
Mamata Parhi

Dynamics of New Technology

Diffusion; A Study of the Indian

Automotive Industry

UNU-MERIT Dissertation Series, № 45 
Andreas Reinstaller

Social Structures and the Innovation

Process; Their Role in the Demand of

Firms and Consumers

UNU-MERIT Dissertation Series, № 44

Rose Kiggundu

Innovation systems and Development;

The Journey of a Beleaguered Nile

Perch Fishery in Uganda

UNU-MERIT Dissertation Series, № 43

Thomas Pogue

The Evolution of Research

Collaboration in South African Gold

Mining; 1886-1933

UNU-MERIT Dissertation Series, № 42

\section{5}

Samia Satti Osman Mohamed-Nour

Change and Skill Development in the Arab Gulf Countries

UNU-MERIT Dissertation Series, № 39

Elad Harison

Intellectual Property Rights;

Economics and Policy Analysis

UNU-MERIT Dissertation Series, № 38
Geoffrey Gachino

Foreign Direct Investment, Spillovers

and Innovation; The Case of Kenyan

Manufacturing Industry

UNU-MERIT Dissertation Series, № 41

Önder Nomaler

Technological Change, International

Trade and Growth; An Evolutionary, Multi-Agents-Based Modeling

Approach

UNU-MERIT Dissertation Series, № 40

Daniel Dalohoun

The Relationship between RED

Partnership Formation, Social

Embeddedness and Innovative

Performance; a Multi-level Approach of Social Embeddedness

UNU-MERIT Dissertation Series, № 37

Müge Ozman

Networks, Organizations and

Knowledge

UNU-MERIT Dissertation Series, № 36 
Bas Straathof

Product Variety and Economic

Growth; The Counteracting Effects of

Scale and Idiosyncrasy

UNU-MERIT Dissertation Series,

№ 35

Wilfred Schoenmakers

Knowledge Flows between

Multinational Companies; A Patent

Data Analysis

UNU-MERIT Dissertation Series,

№ 34

2004

Paola Criscuolo

$R \mathcal{E D}$ Internationalisation and

Knowledge Transfer; Impact on MNEs

and their Home Countries

UNU-MERIT Dissertation Series,

№ 32

Maarten Verkerk

Trust and Power on the Shop Floor

UNU-MERIT Dissertation Series,

№ 31
Myriam Cloodt

Mergers and Acquisitions ( $M$ and As)

in High-Tech Industries; Measuring

the Post- $M$ and $A$ Innovative

Performance of Companies

UNU-MERIT Dissertation Series, № 33
Gottfried Leibbrandt

Adoption, Harmonization and

Succession of Network Technologies across Countries

UNU-MERIT Dissertation Series, № 30

Mark Sanders

Skill Biased Technical change; Its

Origins, the Interaction with the

Labour Market and Policy Implications

UNU-MERIT Dissertation Series, № 29

2003

Nadine Roijakkers

Inter-firm Cooperation in High-tech Industries; a Study of RED

Partnerships in Pharmaceutical

Biotechnology

UNU-MERIT Dissertation Series, № 28
Viki Sonntag

Speed, Scale and Sustainability UNU-MERIT Dissertation Series, № 27 
Masaru Yarime

From End-of-Pipe Technology to Clean

Technology

UNU-MERIT Dissertation Series, № 26
Stéphane Malo

The Combinatorial Chemistry

Revolution; Sustaining a Superior

Performance Position through

Technological Learning

UNU-MERIT Dissertation Series, № 25

Bastiaan Johan ter Weel

The Computerization of the Labour Market

UNU-MERIT Dissertation Series

UNU-MERIT Dissertation Series, № 24

2001

John Adeoti

Technology Investment in Pollution Control in Sub-Saharan Africa; The

Case of the Nigerian Manufacturing Industry

UNU-MERIT Dissertation Series, № 23

2000

Machiel van Dijk

Technological Change and the

Dynamics of Industries; Theoretical Issues and Empirical evidence from

Dutch Manufacturing

UNU-MERIT Dissertation Series, № 21
Edward Huizenga

Innovation Management; How

Frontrunners Stay Ahead; An

Empirical Study on Key Success

Factors in the ICT sector

UNU-MERIT Dissertation Series, № 22 
Jan Cobbenhagen

Managing Innovation at the Company

Level; A Study on Non-Sector-Specific

Success Factors

UNU-MERIT Dissertation Series,

№ 20

1998

\section{Aldo Geuna}

Resource Allocation and Knowledge

production; Studies in the Economics

of University Research

UNU-MERIT Dissertation Series,

№ 18

1996

Reinoud Joosten

Dynamics, Equilibria, and Values

UNU-MERIT Dissertation Series, № 17

\section{5}

Hans van Meijl

Endogenous Technological Change;

The Case of Information Technology,

Theoretical Considerations and

Empirical Results

UNU-MERIT Dissertation Series, № 15
Marjolein Caniëls

Regional Growth Differentials; The Impact of Locally Bounded Knowledge Spillovers

UNU-MERIT Dissertation Series, № 19
Hugo Kruiniger

Investment, $R \mathcal{E} D$, and the Financing

Decisions of the Firm

UNU-MERIT Dissertation Series, № 16

\section{René Kemp}

Environmental Policy and Technical

Change; A Comparison of the

Technological Impact of Policy

Instruments

UNU-MERIT Dissertation Series, № 14 
Rohini Acharya

The Impact of New Technologies on

Economic Growth and Trade; A Case

Study of Biotechnology

UNU-MERIT Dissertation Series,

№ 13

Geert Duysters

The Evolution of Complex

Industrial Systems; The Dynamics

of Major IT Sectors

UNU-MERIT Dissertation Series,

№ 12

1994

Huub Meijers

On the Diffusion of Technologies in a

Vintage Framework; Theoretical

Considerations and Empirical Results

UNU-MERIT Dissertation Series,

№ 10

Theon van Dijk

The Limits of Patent Protection; Essays

on the Economics of Intellectual

Property Rights

UNU-MERIT Dissertation Series,

№ 9

1993

Paul Diederen

Technological Progress in Enterprises and Diffusion of Innovation;.

Theoretical Reflections and Empirical

Evidence

UNU-MERIT Dissertation Series,

№ 7
Marjan Groen

Technology, Work and Organisation;

A Study of the Nursing Process in Intensive Care Units

UNU-MERIT Dissertation Series, № 11

Hans Voordijk

Naar Integrale Logistiek in

Bedrijfsketens; Ontwikkelingen in de

Bouw

UNU-MERIT Dissertation Series, № 8

Ben Dankbaar

Economic Crisis and Institutional

Change; The Crisis of Fordism from the Perspective of the Automobile Industry UNU-MERIT Dissertation Series, № 6 
Hanno Roberts

Accountability and Responsibility; The

Influence of Organisation Design on

Management Accounting

UNU-MERIT Dissertation Series,

№ 5

1992

Bart Verspagen

Uneven Growth Between

Interdependent Economies; An

Evolutionary View on Technology

Gaps, Trade and Growth

UNU-MERIT Dissertation Series,

№ 4

1989

John Spangenberg

Economies of Scale, and Atmosphere in

Research Organisations

UNU-MERIT Dissertation Series,

№ 2

1988

John Hagedoorn

Evolutionary and Heterodox

Innovation Analysis; A Study of

Industrial and Technological

Development in Process Control and

Information Technology

UNU-MERIT Dissertation Series,

№ 1
Sjoerd Romme

A Self-organization Perspective on

Strategy Formation

UNU-MERIT Dissertation Series, № 3 